\title{
Alternative Routen in komplexen Umgebungen
}

Sebastian Feld

\begin{abstract}
Dissertation
an der Fakultät für Mathematik, Informatik und Statistik der Ludwig-Maximilians-Universität München
\end{abstract}

vorgelegt von

Sebastian Feld

Tag der Einreichung: 18. Dezember 2017 



\title{
Alternative Routen in komplexen Umgebungen
}

Sebastian Feld

\begin{abstract}
Dissertation
an der Fakultät für Mathematik, Informatik und Statistik der Ludwig-Maximilians-Universität München
\end{abstract}

\author{
vorgelegt von \\ Sebastian Feld
}

1. Berichterstatter: Prof. Dr. Claudia Linnhoff-Popien

2. Berichterstatter: Prof. Dr. Uwe Baumgarten

Tag der Einreichung: $\quad$ 18. Dezember 2017

Tag der Disputation: 08. Mai 2018 



\section{Eidesstattliche Versicherung}

(siehe Promotionsordnung vom 12.07.11, § 8, Abs. 2 Pkt. 5)

Hiermit erkläre ich an Eides statt, dass die Dissertation von mir selbstständig, ohne unerlaubte Beihilfe angefertigt ist.

München, 15. Mai 2018

Sebastian Feld 



\section{Zusammenfassung}

Durch die immense Verbreitung kostengünstiger GPS-Empfänger, eingebaut in mobile Endgeräte, wurde in den letzten Jahren eine beeindruckend starke Nutzung von ortsbezogenen Anwendungen und Diensten erreicht. Ein beliebter Anwendungsfall ist die Navigation im Straßenverkehr zusammen mit der Präsentation von alternativen Routen, die dem Anwender eine Auswahl nach eigenen Präferenzen oder Erfahrungen ermöglicht.

Die Wegefindung in komplexen Umgebungen unterscheidet sich von der in Straßennetzen hauptsächlich durch die Tatsache, dass sich ein Anwender nahezu in alle Richtungen bewegen kann. Beispiele hierfür sind Fußgänger in Flughäfen, Krankenhäusern, Messehallen oder Parks, mobile Roboter in Industrieanlagen oder Lagerhallen, sowie Nicht-Spieler-Charaktere in Computerspielen. Auch in diesen Szenarien ist das Vorhalten von alternativen Routen sinnvoll, um beispielsweise eine personalisierte Navigation zu ermöglichen, proaktiv Stau zu vermeiden oder taktische Bewegungen durchzuführen.

In der vorliegenden Arbeit werden Ansätze und Verfahren vorgestellt, die das Thema der alternativen Routen in komplexen Umgebungen auf unterschiedlichen thematischen Ebenen behandelt. Darunter fallen die Berechnung alternativer Routen in Freiflächen, der Vergleich geospatialer Trajektorien, sowie die Identifizierung von Strukturen in Gebäuden.

Im ersten Teil der Arbeit werden alternative Routen in komplexen Umgebungen mittels des topologischen Konzepts der Homotopie definiert, sodass zwei Routen als Alternativen zueinander angesehen werden, wenn sie Hindernisse unterschiedlich umlaufen. Hierzu wird eine effiziente Annäherung des Tests auf Homotopie vorgestellt und es werden zwei Algorithmen zum Berechnen solcher Routen implementiert. Anschließend findet eine strukturierte Darstellung bestehender Qualitätsmetriken für alternative Routen und Alternativgraphen in Straßennetzen statt, woraufhin die Übertragbarkeit dieser Ansätze auf komplexe Umgebungen diskutiert wird.

Im zweiten Teil der Arbeit werden Ansätze zum direkten Vergleich von Routen vorgestellt. Einerseits wird ein Scoring von Routen basierend auf der Annahme vorgeschlagen, dass Routen, die oft auf einem kürzesten Pfad liegen, auch oft durchlaufen werden. Andererseits wird ein System vorgestellt, das die Berechnung archetypischer Routen ermöglicht, indem es aus einer Menge von Routen die extremsten Exemplare extrahiert. Korrespondierend dazu wird die archetypische Distanz definiert, mit der Routen nicht nur geometrisch, sondern in einem mehrdimensionalen Merkmalsraum verglichen werden können.

Schließlich wird im dritten Teil der Arbeit die quantitative Analyse der visu- 
ellen Wahrnehmung von Raum in den Kontext alternativer Routen integriert. Basierend auf der Idee sogenannter Isovisten wird die lokale Umgebung eines Anwenders angenähert, um somit alternative Routen zu berechnen, diese zu annotieren, und schließlich Strukturen in Gebäuden zu ermitteln.

Zusammengefasst können die Beiträge der vorliegenden Arbeit in ihrer Gesamtheit als ein Werkzeugkasten verstanden werden, der von weiteren ortsbezogenen Anwendungen und Diensten verwendet werden kann. 


\section{Abstract}

Due to the immense dissemination of low-cost GPS receivers built into mobile devices, an impressive use of location-based services has been achieved in recent years. A popular application is navigation in road networks in conjunction with the presentation of alternative routes that allows users a choice according to their own preferences or experiences.

Route finding in complex environments differs from that in road networks mainly due to the fact that a user can move almost in all directions. Examples include pedestrians in airports, hospitals, exhibition halls, or parks, mobile robots in industrial facilities or warehouses, as well as non-player characters in computer games. In these scenarios the provision of alternative routes is useful, too, for example, to enable personalized navigation, to avoid jams proactively, or to carry out tactical movements.

This thesis presents approaches which deal with the topic of alternative routes in complex environments at different thematic levels. This includes the calculation of alternative routes in open space, the comparison of geospatial trajectories, and the identification of structures in buildings.

First, alternative routes in complex environments are defined using the topological concept of homotopy, so that two routes can be considered alternative if they pass obstacles differently. For this purpose an efficient approximation of the homotopy test is presented together with the implementation of two algorithms for the calculation of such routes. Subsequently a structured presentation of existing quality metrics for alternative routes and alternative graphs in road networks takes place, whereupon the transferability of these approaches into complex environments is discussed.

Second, approaches for the direct comparison of routes are presented. On the one hand, a scoring of routes is suggested based on the assumption that routes, which often lie on a shortest paths, are also often traversed. On the other hand, a system is presented that allows the calculation of archetypal routes by extracting the most extreme instances from a set of routes. Corresponding to this, the archetypal distance is defined, with which routes can be compared not only geometrically but in a multi-dimensional feature space.

Third, the quantitative analysis of the visual perception of space is incorporated into the context of alternative routes. Based on the idea of so-called Isovists, a user's local environment is approximated in order to calculate alternative routes, to annotate them, and to determine structures in buildings.

In summary, the contributions of this thesis can be understood in their entirety as a toolbox which can be used by other location-based services. 


\section{Inhaltsverzeichnis}

1 Einleitung $\quad 1$

1.1 Motivation und Beiträge der Arbeit . . . . . . . . . . . . 3

1.2 Vorveröffentlichungen . . . . . . . . . . . . . . . . 6

1.3 Aufbau der Arbeit . . . . . . . . . . . . . . . . 9

2 Grundlagen der Navigation in komplexen Umgebungen 11

2.1 Ortsbezogene Anwendungen und Dienste . . . . . . . . . . . . 11

2.1.1 Historie und Begriffserklärung . . . . . . . . . . . . 11

2.1.2 Anwendungskategorien . . . . . . . . . . . . . 13

2.1.3 Abgrenzung. . . . . . . . . . . . . . . . . . . . 14

2.2 Alternative Routen . . . . . . . . . . . . . . . 15

2.2.1 Begriffsdefinitionen . . . . . . . . . . . . 15

2.2.2 Lösungen für das Kürzester-Pfad-Problem . . . . . . . . . . 16

2.2.3 Erweiterte Problemstellungen . . . . . . . . . . . . . 17

2.2.4 Alternative Routen in der Anwendung . . . . . . . . . . . . . 18

2.3 Komplexe Umgebungen . . . . . . . . . . . . . . . . . . . . . 19

2.3.1 Definition komplexer Umgebungen . . . . . . . . . . . . 19

2.3.2 Kartendarstellungen . . . . . . . . . . . . . . . 21

2.3.3 Ortsbezogene Dienste in komplexen Umgebungen . . . . . . . 23

2.4 Anwendungsfälle für alternative Routen in komplexen Umgebungen 25

2.5 Zusammenfassung und Ausblick .............. 26

3 Berechnung alternativer Routen in Freiflächen 29

3.1 Motivation und Herausforderungen . . . . . . . . . . . . . . . . . . 29

3.2 Definition alternativer Routen mittels Homotopie . . . . . . . . . . 31

3.2.1 Homotopie als Äquivalenzrelation . . . . . . . . . . . . . . . 32

3.2 .2 Approximation der Homotopie . . . . . . . . . . . . . 36

3.3 Heuristiken zum Finden nicht-homotoper Pfade . . . . . . . . . . 39

3.3.1 One-Patching-Algorithmus . . . . . . . . . . . . 40

3.3 .2 Penalty-Algorithmus . . . . . . . . . . . . . . . . 42

3.3.3 Evaluation der Heuristiken zum Finden nicht-homotoper Pfade 43

3.3.4 Zusammenfassende Bewertung ... . . . . . . . . . 49

3.4 Auswahlkriterien für Alternativen in Straßennetzen . . . . . . . . 52

3.4.1 Qualitätsmetriken für alternative Routen . . . . . . . . 53

3.4.2 Qualitätsmetriken für Alternativgraphen . . . . . . . . . 55

3.5 Übertragbarkeit auf alternative Routen in Freiflächen . . . . . . . . 57

3.5.1 Limited Sharing und Total Distance . . . . . . . . . . 58 
3.5.2 Uniformly Bounded Stretch und Average Distance . . . . . . 62

3.5.3 Local Optimality . . . . . . . . . . . . . . . 63

3.5.4 Decision Edges . . . . . . . . . . . . . . . 64

3.5.5 Gegenseitige Beeinflussung der Qualitätsmetriken . . . . . . 66

3.6 Zusammenfassung . . . . . . . . . . . . . 6 66

$4 \quad$ Vergleich geospatialer Trajektorien $\quad 69$

4.1 Motivation und Herausforderungen . . . . . . . . . . . . . 70

4.2 Clusteranalyse und Distanzmaße . . . . . . . . . . . . . . 71

4.2.1 Clustering-Algorithmen . . . . . . . . . . . . 72

4.2.2 Archetypische Analyse . . . . . . . . . . . . . . 74

4.2 .3 Distanzmaße . . . . . . . . . . . . . . . . 77

4.3 Überlastungswahrscheinlichkeit zur Bewertung von Routen . . . . . 79

4.3.1 Definitionen und Ziel . . . . . . . . . . . . . . . . . . 81

4.3.2 Überlastungswahrscheinlichkeit . . . . . . . . . . . . . 81

4.3.3 Methodik . . . . . . . . . . . . . . . . 84

4.3.4 Evaluation und Diskussion . . . . . . . . . . 88

4.3.5 Zusammenfassende Bewertung . . . . . . . . . . . . . . . 91

4.4 Archetypische Routen und Archetypische Distanz . . . . . . . . . . 92

4.4.1 Archetypen von alternativen Routen . . . . . . . . . . . 93

4.4.2 Archetypische Distanz als Distanzmaß für Trajektorien . . . . 96

4.4.3 System zur Berechnung archetypischer Routen . . . . . . . 97

4.4.4 Evaluation . . . . . . . . . . . . . . . . . 99

4.5 Zusammenfassung . . . . . . . . . . . . . . 111

$5 \quad$ Identifizierung von Strukturen in Gebäuden 113

5.1 Motivation und Herausforderungen . . . . . . . . . . . . . 113

5.2 Einbeziehung der lokalen Umgebung mittels Isovisten . . . . . . . . 115

5.2.1 Quantitative Analyse der Wahrnehmung von Raum . . . . . . 116

5.2 .2 Analyse von Isovisten . . . . . . . . . . . . . . . 118

5.3 Diskrete Isovisten als Approximation der lokalen Umgebung . . . . 123

5.3.1 Definition und Berechnung diskreter Isovisten . . . . . . . . . 123

5.3 .2 Evaluation . . . . . . . . . . . . . . . 126

5.4 Archetypische Routen und Regionen unter Einbeziehung der loka-

len Umgebung . . . . . . . . . . . . . . . 136

5.4 Konzept . . . . . . . . . . . . . . . . 136

5.4 .2 Evaluation und Diskussion . . . . . . . . . . . 138

5.5 Zusammenfassung . . . . . . . . . . . . . . . 149

6 Zusammenfassung und Ausblick 153

6.1 Zusammenfassung . . . . . . . . . . . . . . . . . . 154

6.2 Ausblick . . . . . . . . . . . . . . . . 156

$\begin{array}{ll}\text { Literaturverzeichnis } & 159\end{array}$ 


\section{Danksagung}

Die vorliegende Dissertation ist während meiner Zeit am Lehrstuhl für Mobile und Verteilte Systeme an der Ludwig-Maximilians-Universität in München entstanden. Dabei hatte ich das Glück von vielen Seiten Unterstützung in ganz unterschiedlicher Form zu erfahren. Auf diesem Wege möchte ich meinen tiefsten Dank aussprechen.

Allen voran möchte ich mich bei meiner Doktormutter Prof. Dr. Claudia Linnhoff-Popien bedanken. Sie hat mir vor beinahe sechs Jahren die Möglichkeit eröffnet an ihrem Lehrstuhl zu promovieren und mir vom ersten Tag an ihr Vertrauen geschenkt. Ich möchte mich insbesondere für die Offenheit bedanken und für die Möglichkeit, dass ich stets alles ansprechen konnte und viele Freiheiten hatte. Ich habe dadurch außerordentlich viel gelernt.

Darüber hinaus gilt mein Dank Prof. Dr. Uwe Baumgarten für die Übernahme der Zweitberichterstattung sowie Prof. Dr. Christian Böhm für das Mitwirken als Vorsitz der Prüfungskommission.

Ebenso möchte ich mich bei meinen Kollegen und Freunden am Lehrstuhl bedanken. Hervorzuheben ist das außerordentlich freundschaftliche, produktive und fachlich fundierte Arbeitsklima. Es hat einfach Spaß gemacht, mit den unterschiedlichsten Persönlichkeiten unzählige Gespräche und Diskussionen zu führen, gemeinsam zu publizieren oder Vorträge zu halten, Projekte zu bearbeiten, Studenten zu betreuen oder Lehrveranstaltungen durchzuführen.

Jede einzelne Person am Lehrstuhl hat mich auf ganz unterschiedliche Art - sei es fachlich oder moralisch - unterstützt. Besonders hervorheben möchte ich drei Personen: Dr. Martin Werner hat mir geholfen den Grundstein der vorliegenden Dissertation zu legen und stand mir darüberhinaus mit seiner scharfsinnigen und intelligenten Art in vielen Bereichen zur Seite. Marie Kiermeier danke ich insbesondere für die Unterstützung bei der präzisen Formulierung meiner Gedanken sowie für die Möglichkeit, dass ich ihr jederzeit blind vertrauen konnte. Bei Dr. Mirco Schönfeld möchte ich mich für wahre Freundschaft bedanken und für all das, was sich daraus entwickelt.

Schließlich gehört mein größter Dank meinen Eltern und meiner Schwester. Ich möchte mich für das unendliche Vertrauen bedanken, für die offenen Ohren und das Verständnis, für die Liebe und den fehlenden Zweifel. Danke für den unbeirrten Fokus auf das Gute. 


\section{Einleitung}

Umwege erweitern die Ortskenntnis.

- Kurt Tucholsky

Intelligente Mobilität ist eines der großen Schlagworte der heutigen Zeit. Nicht nur die deutsche Bundesregierung sieht sie in ihrer neuen HightechStrategie als eine der prioritären Zukunftsaufgaben [24], [25], auch wird das Thema als eine von drei Fokusgruppen innerhalb der Plattform „Digitale Netze und Mobilität" des Digital-Gipfels (ehemals Nationaler IT-Gipfel) behandelt [54], [55]. Forschungsergebnisse aus den unterschiedlichsten Themenfeldern wie etwa Mobilkommunikation, Batterietechnik oder Algorithmen und Datenstrukturen, um nur einige Beispiele zu nennen, finden in der intelligenten Mobilität eine integrierte Anwendung und haben somit direkten Einfluss auf das Leben der einzelnen Bürger und auf die Gesellschaft im Allgemeinen. Voraussetzung für eine moderne Mobilität ist das Vorhandensein von Daten, um den aktuellen Zustand messen und entsprechend reagieren zu können. Somit bekommt das oft zitierte Internet der Dinge ein Gesicht. Die Ideen hinter der intelligenten Mobilität verfolgen das Ziel der Effizienz, was im Sinne eines Optimierungsproblems ein ansprechendes Thema für die Informatik ist.

Eine enorm wichtige Komponente bei der Realisierung von Visionen wie etwa dem automatisierten Fahren [72] sind digitale Karten. Diese dreidimensionalen Karten [96] sollten möglichst hoch aufgelöst und mit Echtzeitinformationen angereichert sein. Eine bemerkenswerte Aktion hat 2015 stattgefunden, als die drei deutschen Automobilhersteller Audi, BMW und Daimler den Geokartendienst Here übernommen haben [144].

Nicht nur die Techniken im Hintergrund werden immer intelligenter, sondern auch die Anwendungen, die im direkten Kontakt zum Menschen stehen. Smartphones sind die ständigen Begleiter der Menschen [168] und werden nicht selten für Anwendungen mit Ortsbezug verwendet [163]. Über Spracheingabe werden dem Smartphone Fragen wie etwa „Wo ist der nächste Bankautomat?“ oder „Wann sollte ich am besten losfahren, um trotz Stau rechtzeitig anzukommen?" gestellt. Zunehmend werden ortsbezogene Anwendungen und Dienste nicht nur im Freien oder im Kontext von Straßennetzen, sondern auch innerhalb von Gebäuden verwendet. Dies passt zu einer bereits im Jahre 2001 veröffentlichten Studie, die besagt, dass Menschen aus den USA mehr als 80 Prozent ihrer Zeit innerhalb von Gebäuden verbringen [23], [123]. Aber nicht nur der Mensch profitiert von den Anwendungen, sondern auch Anwendungen 
von den Menschen. Aktuelle Themen in der Forschungsgemeinde der ortsbezogenen Anwendungen und Dienste sind insbesondere crowdsourcing sowie volunteered geographic information (VGI), also der Rückkanal des Menschen zur Anwendung [149], [170].

Ein eigener Forschungsbereich ist das Thema Robotik. Dies ist gleichzeitig auch integraler Bestandteil der Visionen einer intelligenten Mobilität innerhalb von Smart Cities mit Smart Factories. In sich selbst organisierenden Industrieanlagen soll das System selbst entscheiden, welchen Weg die Werkstücke bei der Produktion nehmen und in welcher Reihenfolge von welchen Maschinen diese verarbeitet werden sollen. Neuartige Fragestellungen beziehen sich somit auf das Testen der Qualität eines solchen Systems [59] sowie auf die Ergründung von Fehler(-ketten) [166]. Auch in der Logistik werden zunehmend autonome Roboter eingesetzt. So verwendet ein chinesisches Lieferunternehmen einen Roboterschwarm um Pakete zu sortieren [167] und auch in den Warenhäusern des Online-Versandhändlers Amazon werden in großem Umfang Roboter verwendet [95], [157]. Schließlich finden regelmäßig Wettkämpfe für Roboter statt, in denen es um das autonome Spielen von Fußball geht, um das eigenständige Suchen nach Überlebenden bei der Katastrophenhilfe oder um das Erfüllen unterschiedlicher Aufgaben im täglichen Leben innerhalb einer Wohnung [4], [122].

Ermöglicht werden die oben aufgeführten Innovationen durch die geschickte Einbeziehung und die intelligente Verarbeitung von ortsbezogenen Daten. Der Forschungsbereich Spatial Computing umfasst grundsätzlich alle Ideen und Techniken, die sich auf das Verständnis, die Kommunikation und die Visualisierung von Ortsinformationen beziehen [163], [187]. Um etwas abstrahierter und technischer zu werden: Grundlage für die erwähnten Themen ist stets eine passende Abbildung und Behandlung der realen Welt. In einem ergänzendem Video [31] zur Titelgeschichte Spatial Computing [163] der Fachzeitschrift Communications of the ACM wurde folgendes Bild beschrieben:

Ein Punkt ist einzigartig; eine Linie ist die kürzeste Distanz zwischen Punkten; eine Ebene ist eine perfekte Oberfläche und einfache Koordinaten beschreiben eine Kugel. Die Berechnung räumlicher Zusammenhänge in dieser imaginären Welt ist einfach. Aber die Realität unserer Welt spricht dagegen. Der Globus wölbt sich in der Mitte, die Erdoberfläche besitzt Berge und Täler, und Straßen schlängeln sich.

Es herrscht also eine Diskrepanz zwischen der Theorie und der Realität. Es werden Ansätze benötigt, die die Unsicherheiten der realen Welt für den Computer greifbar machen. Es herrscht stets ein Kompromiss, einerseits wird eine Abstraktion benötigt, andererseits jedoch eine Genauigkeit. Diese Gedanken stellen die Ausgangsbasis für die vorliegende Arbeit dar. 


\subsection{Motivation und Beiträge der Arbeit}

Die vorliegende Arbeit befasst sich mit alternativen Routen in komplexen Umgebungen. Selbst ohne eine genauere Beschreibung des Begriffs ,komplexe Umgebung“" scheinen ,alternative Routen“ einen generellen Zweck zu besitzen: sie bieten die Möglichkeit zur Entscheidung. Bevor jedoch alternative Routen beziehungsweise Alternativen im Allgemeinen verwendet werden können, müssen verschiedene Fragen beantwortet werden. Die grundlegendste Frage ist zum einen, ab wann eine Möglichkeit nicht mehr äquivalent ist, sondern alternativ. Zum anderen stellt sich die Frage nach der qualitativen Vergleichbarkeit von Entscheidungsmöglichkeiten. In diesem Abschnitt werden die Fragestellungen, die diese Arbeit motivieren, vorgestellt und die Beiträge der entsprechenden Kapitel benannt.

Die grundsätzliche Motivation der Arbeit ist die Vorstellung des Besuchs eines Flughafens. Dieser Ort ist ein perfektes Beispiel für eine komplexe Umgebung, in der es weitläufige Freiflächen gibt, in denen man sich nahezu frei bewegen kann. Im Sinne der Routenfindung ist beim Besuch eines Flughafens oft auch ein Start- und ein Zielpunkt gegeben. Der Startpunkt für das Durchlaufen eines Flughafens kann das Parkhaus sein, einer der vielen Eingänge in das Gebäude, der Check-in-Schalter einer Fluggesellschaft oder schlichtweg der aktuelle Standpunkt. Das Ziel der eigenen „Route“ kann ein genau zu benennendes Gate im Falle einer Reise oder der Ankunftsbereich, falls jemand abgeholt wird, sein. Darüber hinaus gibt es zahlreiche Möglichkeiten (Alternativen), das Ziel zu erreichen. Bei wenig verbleibender Zeit sollte der schnellste Weg verwendet werden, der nicht zwingend auch der kürzeste Weg sein muss. Falls bis zum Abflug noch viel Zeit vorhanden ist, so sind Routen denkbar, die an Geschäften, Souvenir-Ständen, Bistros oder Toiletten vorbei führen. Schließlich sind zudem Entscheidungsmöglichkeiten dadurch gegeben, dass unterschiedliche Check-in-Schalter der Fluggesellschaft verwendet werden können, verschiedene Warteschlangen bei der Kontrolle des Handgepäcks und zahlreiche Wege innerhalb der Duty-Free-Shops. Aber auch die Verwendung von Treppen, Rolltreppen, Aufzügen oder horizontalen Fahrsteigen bietet Möglichkeiten zur Auswahl.

Auf einer abstrakten Ebene können Alternativen über eine Art Semantik beschrieben werden: „nimm den Aufzug“, „,nach der Sicherheitskontrolle rechts halten“ oder „am besten durchläufst du den Duty-Free-Shop“. Erstes Ziel der vorliegenden Arbeit ist es demnach, eine algorithmische Berechnung alternativer Routen in Freiflächen (siehe Kapitel 3) zu ermöglichen. Im ersten Schritt wird eine Definition vorgestellt, mit der entschieden werden kann, ob eine Route alternativ oder äquivalent $\mathrm{zu}$ einer anderen anzusehen ist. Dazu wird das topologische Konzept der Homotopie verwendet (Abschnitt 3.2.1). Vereinfacht ausgedrückt gelten zwei Routen mit gleichem Start- und Zielpunkt als äquivalent, wenn sie die Hindernisse in der gleichen Art und Weise umlaufen. Anschließend wird eine effiziente Methode zur Berechnung dieser Abfrage vor- 
gestellt (3.2.2). Diese Methode bezieht sich auf Routen, die als Vektoren von $x$ - und $y$-Koordinaten dargestellt sind und in einer monochromen Karte eingezeichnet sind. Die Idee hinter der Methode ist das Überprüfen, ob die von den Routen eingeschlossene Fläche Hindernisse beinhaltet oder nicht. Ferner werden Heuristiken vorgestellt, mit denen die alternativen Routen für gegebene Start- und Zielpunkte innerhalb einer Karte berechnet werden können (3.3). Die Heuristiken werden zudem zur Berechnung von Mengen von Routen verwendet, die als Eingabe für weitere in dieser Arbeit vorgestellte Ansätze dienen. Neben der Frage nach der Definition einer alternativen Route ist eine weitere Motivation des Kapitels die Frage nach der Qualität einer Route. So wird in Abschnitt 3.4 untersucht, wie sich die Ansätze der umfangreichen Literatur bezüglich Qualitätsmetriken von alternativen Routen und Alternativgraphen in Straßennetzen im Kontext von komplexen Umgebungen verhalten. Ein Beitrag dieser Arbeit ist die strukturierte Aufbereitung des Stands der Technik bezüglich Auswahlkriterien für Alternativen in Straßennetzen sowie insbesondere die Übertragbarkeit auf Freiflächen (3.5). Die Philosophie dahinter ist die Entwicklung und Diskussion von Qualitätsmetriken in komplexen Umgebungen.

Eine weitere Motivation dieser Arbeit und Basis für die angesprochenen Qualitätsmetriken ist die Frage, wie Routen, die durch ein Gebäude führen, angemessen verglichen werden können. Wie eingangs erwähnt sind einfache geometrische Anfragen in der Theorie eindeutig: die Distanz zwischen zwei Punkten ist die Länge der verbindenden Linie. Wenn nun aber anstatt zweier Punkte zwei Folgen von Punkten betrachtet werden, wie steht es dann um die Distanz zwischen diesen geospatialen Trajektorien? Als motivierendes Beispiel sollen vier exakt parallele Linien dienen: die Intuition besagt, dass die beiden äußeren Linien weiter voneinander entfernt sind als die beiden Inneren. Nun stellt sich die Frage, wie die Distanz zwischen zwei sich kreuzenden Linien ist oder zwischen Linien, die stets nah zueinander verlaufen, nur an einer Stelle einen Ausreißer haben, an denen sie sich voneinander entfernen und anschließend wieder nähern. Der Begriff der Distanz, sei es zwischen zwei Routen oder generell zwischen zwei Objekten, kann jedoch auch viel weiter gefasst werden. Die Frage nach Gleichheit kann sehr subjektiv oder gar philosophisch beantwortet werden, sie bezieht sich nicht selten auf den Kontext oder beinhaltet Einschätzungen, die schwer oder gar nicht algorithmisch mess- oder verarbeitbar sind.

Dieser grundsätzlichen Problematik widmen sich die Beiträge zum Vergleich geospatialer Trajektorien (siehe Kapitel 4). Zuerst wird für eine gegebene Menge von Routen durch ein Gebäude ein Scoring erstellt. Das Scoring ist eine Technik, um schwer abzuschätzende Sachverhalte wie etwa qualitative oder subjektive Fakten (beispielsweise die Kreditwürdigkeit) vergleichbar zu machen. Dazu werden Punkte berechnet und den Sachverhalten zugeordnet, wodurch diese sortierbar werden. Die Idee hinter der vorzustellenden Bewertungsfunktion von Routen ist die, dass Orte innerhalb einer Karte, die häufig auf 
einem kürzesten Weg liegen, auch häufig durchlaufen werden. Dadurch wird eine Überlastungswahrscheinlichkeit für Regionen innerhalb einer Karte erstellt, die ausschließlich auf Kürzester-Pfad-Anfragen basiert. Die zu vergleichenden Routen werden anhand der von ihnen durchlaufenen Regionen als potentiell verkehrsarm oder verkehrsreich bewertet und sortiert. Darüber hinaus ist ein Vergleich von Routen auch aufgrund der Tatsache notwendig, dass ein Gebäudeplan, in dem eine Navigation notwendig ist, gleichzeitig auch eine hohe Anzahl von alternativen Routen beinhaltet - zumindest nach der oben erwähnten Definition. Es ist jedoch das Extrahieren von möglichst wenigen, möglichst unterschiedlichen Routen anzustreben. Ein weiterer Beitrag der vorliegenden Arbeit ist ein System, mit dem solche extremen Beobachtungen, also ursprüngliche, originale oder archetypische Routen, erstellt werden können. Im Zuge der Erstellung dieses Systems wurde zudem ein neuartiges Distanzmaß namens archetypische Distanz definiert, mit dem Routen basierend auf ihren zahlreichen Eigenschaften untersucht werden können.

Schließlich ist die vorliegende Arbeit durch die Idee motiviert, dass eine Route erst durch die Karte, in der sie eingebettet ist, eine Bedeutung wie etwa äquivalent oder alternativ erhält. Karten beziehungsweise die dargestellten Gebäude oder räumlichen Umgebungen enthalten Informationen, die sich nach einer Extraktion auf die Route übertragen lassen. Wenn ein Weg beschrieben wird, so kann man sich mittels Ausdrücken wie „einfach durch die Haupthalle“ oder „,kurz nach dem Eingangsbereich rechts halten“ artikulieren. Es scheint also funktionell oder anderweitig eingeteilte, abgegrenzte Bereiche zu geben. Darüber hinaus werden solche funktionellen Bereiche unterbewusst in Zusammenhang mit der Wahrnehmung des Raums gebracht, oder anders ausgedrückt: bestimmte wiederkehrende Eigenschaften visueller Wahrnehmung werden bewusst verwendet. Ein einfaches Beispiel ist die Platzierung von Feuerlöschern: diese sollten vorzugsweise an Kreuzungspunkten von Verkehrswegen angebracht und von möglichst vielen Seiten gut sichtbar sein. Im Umkehrschluss bedeutet das, dass Menschen intuitiv den Standort des nächstgelegenen Feuerlöschers richtig vermuten. Die Idee hinter dem dritten Teil der vorliegenden Arbeit ist es, den Gedanken der räumlichen Wahrnehmung in die Berechnung alternativer Routen in komplexen Umgebungen einfließen zu lassen.

Dazu werden in der vorliegenden Arbeit Beiträge vorgestellt, die zur Identifizierung von Strukturen in Gebäuden dienen (siehe Kapitel 5). Im ersten Schritt wurde untersucht, wie die Wahrnehmung von Raum in den generellen Kontext der vorliegenden Arbeit eingesetzt werden kann. Dazu wurden Techniken zur quantitativen Analyse der Wahrnehmung von Raum ausgewertet und das Konzept der Isovisten als passend eingeschätzt. Für eine direkte Verwendung in den in dieser Arbeit vorgestellten Ansätzen wurde eine einfache Annäherung der Isovisten präsentiert und somit die Einbeziehung der lokalen Umgebung im Kontext alternativer Routen in komplexen Umgebungen ermöglicht. Aufbauend darauf wurde eine erste Anwendung dieser diskreten Isovisten gezeigt, 
indem Routen annotiert werden. Die Idee dahinter ist, dass eine Route, die durch ein Gebäude und somit durch einen geografischen Raum führt, unterschiedliche Werte der gemessenen Wahrnehmungseigenschaften besitzt. Diese Empfindungen ändern sich über benachbarte Orte graduell, aber an bestimmten Stellen auch signifikant. Ein Beispiel hierfür ist das Verlassen eines engen Ganges in eine große Halle. Diese Vorstellung lag der Annotation von Routen mittels Isovisten-Messgrößen zugrunde. Schließlich wurde das bereits erwähnte Konzept der archetypischen Analyse dazu verwendet, um besagte Wahrnehmungseigenschaften in Gebäuden zu gruppieren. Es wurden sogenannte archetypische Regionen definiert, die extreme Ausprägungen der gemessenen lokalen Umgebung beschreiben. Schlussendlich wurde untersucht, welchen Einfluss Isovisten-Messgrößen auf die Berechnung archetypischer Routen besitzen, was im Ergebnis zu Routen führt, die ausschließlich unter der Einbeziehung der lokalen Umgebung berechnet werden.

Die Quintessenz der in den drei inhaltlichen Kapiteln vorgestellten Beiträge sind die Definition und Berechnung von alternativen Routen, die Verwendung der archetypischen Distanz zum Vergleich von Routen sowie die Einbeziehung der lokalen Umgebung mittels Isovisten. Das Prinzip der vorliegenden Arbeit ist die Extraktion von Informationen aus einfachen Gebäudeplänen, sodass diese angereichert und veredelt werden. Die Beiträge der Arbeit können in ihrer Gesamtheit als ein Werkzeugkasten verstanden werden, der von bereits existierenden, aber auch zukünftigen Anwendungen oder Diensten verwendet werden kann.

\subsection{Vorveröffentlichungen}

Die Inhalte der vorliegenden Arbeit wurden bereits bei verschiedenen internationalen Konferenzen vorgestellt und in mehreren Veröffentlichungen publiziert. Dieser Abschnitt dient als kurze Vorstellung der jeweiligen Artikel und umreißt den Beitrag der jeweiligen Autoren. Es wird ebenfalls darauf eingegangen, welche Teile der Publikationen in die vorliegende Arbeit übernommen wurden.

Für alle im Folgenden aufgeführten Publikationen gilt, dass Prof. Dr. Claudia Linnhoff-Popien - in ihrer Rolle als Doktormutter des Autors der vorliegenden Arbeit - stets mit konstruktiver Kritik und wertvollen Gedanken mitgewirkt hat. Dies gilt insbesondere dann, wenn sie als Autorin aufgeführt ist.

Homotopy and Alternative Routes in Indoor Navigation Scenarios [179] Die Definition alternativer Routen in komplexen Umgebungen, die Approximation der entsprechenden Äquivalenzrelation sowie die Heuristiken zum Berechnen solcher Pfade wurden bereits in [179] veröffentlicht. Die grundlegende Idee zur Publikation, die Definition mittels Homotopie, die Annäherung der Homotopie sowie der One-Patching-Algorithmus stammen von Dr. Martin 
Werner. Ebenso wurde von Dr. Martin Werner ein initialer Proof of Concept implementiert, der die grundlegende Durchführbarkeit der Idee belegt, und eine Anbindung an eine Graphen-Bibliothek [164] zur Verfügung gestellt. Vom Autor der vorliegenden Arbeit stammt der vorgestellte Penalty-Algorithmus, die abschließende Programmierung sowie die Konzeption, Durchführung und Auswertung der Evaluation. Die Abschnitte Homotopy und Notes on the Implementation der Veröffentlichung [179] sind in stark geänderter und erweiterter Form in Abschnitt 3.2 der vorliegenden Arbeit bezüglich der Definition alternativer Routen eingeflossen. Die Abschnitte Problem Statement und Methodology wurden größtenteils in den Abschnitt 3.3 bezüglich der Heuristiken übernommen, was ebenso für den Abschnitt Experimental Results und den Abschnitt 3.3.3 mit der Evaluation der Heuristiken gilt.

Criteria for Selecting Small Sets of Alternative Routes in Free Space Scenarios [76] Die Literaturauswertung bezüglich Alternativrouten und Alternativgraphen sowie die Überlegungen zur Übertragbarkeit in komplexe Umgebungen wurden bereits in [76] veröffentlicht. Die grundlegende Idee zur Publikation und die Aufbereitung des Standes der Technik bezüglich Alternativrouten und -graphen stammen vom Autor der vorliegenden Arbeit. Die Diskussion der Problematiken sowie der Übertragbarkeit wurden gemeinsam vom Autor der vorliegenden Arbeit und Dr. Martin Werner erarbeitet. Abschnitt Quality Metrics for Alternative Routes der Veröffentlichung [76] wurde quasi vollständig in Abschnitt 3.4 bezüglich der Auswahlkriterien in Straßennetzen der vorliegenden Arbeit übernommen. Ähnlich verhält es sich mit dem Abschnitt Quality Metrics for Alternative Graphs, der um einige Abbildungen erweitert wurde und in den Abschnitt 3.5 bezüglich der Übertragbarkeit eingeflossen ist. Der Abschnitt Map Representations diente als Basis für Abschnitt 2.3.2 im Grundlagenkapitel.

\section{Scoring of Alternative Routes Using Implicit Building Topologies [73]} Die Idee zur Erstellung und Verwendung einer Überlastungswahrscheinlichkeit zur Bewertung von Routen wurde bereits in [73] veröffentlicht. Die grundlegende Idee zur Verwendung der Gewichtskarte (ein Nebenprodukt der Publikation [179]), der Algorithmus, die Programmierung und die Evaluation stammen vom Autor der vorliegenden Arbeit. Dr. Martin Werner hat mit Kritik und Diskussion bei der Formalisierung des Algorithmus und der Erstellung der Publikation beraten. Abschnitt Algorithm der Veröffentlichung [73] ist mit seiner Gedankenfolge und der Erläuterung des Algorithmus in Abschnitt 4.3 der vorliegenden Arbeit bezüglich der Überlastungswahrscheinlichkeit zur Bewertung von Routen übernommen worden. Teile des Abschnitts Discussion sind in die Evaluation in Abschnitt 4.3.4 eingeflossen.

Archetypes of Alternative Routes in Buildings [77] Die Konzepte der archetypischen Routen und der archetypischen Distanz sowie das System zur Be- 
rechnung selbiger wurden bereits in [77] veröffentlicht. Die grundlegende Idee zur Verwendung der archetypischen Analyse im Kontext alternativer Routen, Teile der Implementierung sowie die vollständige Evaluation stammen vom Autor der vorliegenden Arbeit. Die Definition der archetypischen Routen und der archetypischen Distanz wurden gemeinsam vom Autor der vorliegenden Arbeit und Dr. Martin Werner erarbeitet. Darüber hinaus hat Dr. Martin Werner eine effiziente Programmierung der Merkmalserstellung bereitgestellt. Dr. Mirco Schönfeld hat mit Kritik und Diskussion bei der Erstellung der Publikation und insbesondere der Evaluation beraten. Im Vorfeld der Publikation wurde die prinzipielle Durchführbarkeit dieser Idee in einer studentischen Projektarbeit von Stefanie Hasler als Machbarkeitsstudie programmiert [101]. Die studentische Projektarbeit wurde vom Autor der vorliegenden Arbeit betreut, Dr. Mirco Schönfeld war Zweitbetreuer. Abschnitt Route Similarity der Veröffentlichung [77] ist in den Abschnitt 4.2.3 bezüglich existierender Distanzmaße übernommen worden. Abschnitt Archetypal Routes and Distance wurde erweitert und ist in die entsprechenden Abschnitten 4.4.1 und 4.4.2 eingeflossen. Ebenso verhält es sich mit Abschnitt Framework for Calculating Archetypal Routes, welches in Abschnitt 4.4.3 übernommen wurde sowie Abschnitt Evaluation (4.4.4).

Identifying Divergent Building Structures Using Fuzzy Clustering of Isovist Features [74] Das Konzept zur Einbeziehung der lokalen Umgebung mittels Isovisten sowie die Identifizierung archetypischer Regionen und Routen unter Verwendung von Isovisten-Messgrößen wurden bereits in [74] veröffentlicht. Die grundlegende Idee zur Verwendung von Isovisten innerhalb der archetypischen Analyse, die Programmierung des Systems und der Großteil der Evaluation stammen vom Autoren der vorliegenden Arbeit. Hao Lyu (Technische Universität München) hat die Grundlagen zur Analyse der Isovisten und die Programmierung zur Erstellung der exakten Isovisten-Messgrößen bereitgestellt sowie an der Evaluation im Sinne einer Diskussion mitgewirkt. Von Dr. Andreas Keler (Universität Augsburg) stammt ein Großteil der Untersuchung der verwandten Arbeiten, und ebenso hat er im Sinne einer Diskussion an der Evaluation mitgewirkt. Abschnitte Qualitative Perceptual Analysis of Space, Indoor Wayfinding and Navigation in Complex Buildings und Perceptual Analysis and Wayfinding Behavior der Veröffentlichung [74] sind in großen Teilen in Abschnitt 5.2 bezüglich der Einbeziehung der lokalen Umgebung mittels Isovisten eingeflossen, was ebenfalls für Abschnitt Methodology gilt, der in Abschnitt 5.4.1 zur Darstellung des Konzepts archetypischer Regionen und Routen basierend auf Isovisten-Messgrößen verwendet wurde. Abschnitt Results and Discussion wurde bezüglich der Interpretation der Ergebnisse erweitert und hat Einfluss in Abschnitt 5.4.2 gefunden.

Approximated Environment Features With Application to Trajectory Annotation [75] Die Idee zur Erstellung diskreter Isovisten sowie die an- 
schließende Annotation von Routen wurden bereits in [75] veröffentlicht. Die grundlegende Idee der Verwendung von Isovisten-Messgrößen zur Analyse der Gebäudestrukturen, der Algorithmus zur Approximation der Isovisten, die vollständige Programmierung sowie die Evaluation stammen vom Autor der vorliegenden Arbeit. Dr. Martin Werner hat mit Kritik und Diskussion eine Formalisierung der Vorgehensweise und der Verwendung der Messgrößen erwirkt. Hao Lyu (Technische Universität München) hat die exakten Werte und entsprechende Datensätze für die Evaluation der Approximation zur Verfügung gestellt und mit hilfreichen Kommentaren unterstützt. Teile des Abschnitts Concept der Veröffentlichung [75] sind in Abschnitt 5.3.1 bezüglich der Definition und Berechnung diskreter Isovisten übernommen worden. Der Abschnitt Evaluation wurde stark erweitert und dient als Grundlage für den entsprechenden Abschnitt 5.3.2.

\subsection{Aufbau der Arbeit}

Nachdem die grundsätzliche Motivation dieser Arbeit sowie deren Beiträge und Vorveröffentlichungen erläutert wurden, ist der Rest der Arbeit wie folgt strukturiert: In Kapitel 2 wird der Begriff der ortsbezogenen Anwendungen und Dienste eingeführt und erläutert, wieso dies der grundlegende Themenbereich der vorliegenden Arbeit ist. Außerdem werden die Begriffe des Titels, nämlich alternative Routen und komplexe Umgebungen, eingeführt und deren Kombination mit konkreten Anwendungsfällen motiviert.

Kapitel 3 beschreibt eine Definition von alternativen Routen in komplexen Umgebungen sowie Algorithmen zur Berechnung selbiger. Dies stellt das Fundament der vorliegenden Arbeit dar, da weitere Ansätze diese Methoden zur Generierung von Eingabedaten verwenden. Anschließend wird diskutiert, warum der aktuelle Stand der Technik von Qualitätsmetriken im Bereich der Straßennetze nicht uneingeschränkt in komplexen Umgebungen verwendet werden können.

In Kapitel 4 werden Ansätze vorgestellt, die das Ziel haben, eine potentiell hohe Anzahl an Routen in eine möglichst kleine Menge möglichst unterschiedlicher Routen zu verringern. Hierzu wird eine Überlastungswahrscheinlichkeit berechnet, mit denen Routen bewertet und je nach Anwendungsfall sortiert werden können. Anschließend wird ein spezielles Clusteringverfahren - die archetypische Analyse - dazu verwendet, Routen zu gruppieren und je einen Repräsentanten zu wählen. Mit der Definition von archetypischen Routen und der archetypischen Distanz wird somit eine nicht-binäre Beschreibung von alternativen Routen eingeführt.

In Kapitel 5 wird die starke Wechselwirkung zwischen Routen und Karten ausgenutzt, in denen sie eingebettet sind. Es werden Isovisten als geeignete Möglichkeit zur Einbeziehung der lokalen Umgebung identifiziert. Bezugnehmend auf die Isovisten werden Routen annotiert sowie archetypische Regionen und archetypische Routen erstellt. 
Kapitel 6 schließt die vorliegende Arbeit mit einer Zusammenfassung ab und gibt einen Ausblick auf zukünftige Forschungsrichtungen. 


\section{Grundlagen der Navigation in komplexen Umgebungen}

In diesem Kapitel wird zunächst der Begriff der ortsbezogenen Anwendungen und Dienste eingeführt, worunter die in dieser Arbeit behandelten alternativen Routen fallen (Abschnitt 2.1). Es wird abgegrenzt, welche Bereiche solcher Dienste behandelt werden. Anschließend werden die alternativen Routen prosaisch definiert und durch Anwendungsfälle motiviert (Abschnitt 2.2). In der vorliegenden Arbeit werden statt Straßennetze komplexe Umgebungen behandelt, welche zusammen mit ihren unterschiedlichen Kartendarstellungen erläutert werden (Abschnitt 2.3). Es soll vermittelt werden, weshalb alternative Routen auch außerhalb von Straßennetzen verwendet werden können. Schließlich werden konkrete Anwendungsfälle für alternative Routen in komplexen Umgebungen vorgestellt, was die soeben aufgeführten Themenbereiche kombiniert (Abschnitt 2.4).

\subsection{Ortsbezogene Anwendungen und Dienste}

Die vorliegende Arbeit kann dem Forschungsbereich Ortsbezogene Anwendungen und Dienste - auch Standortbezogene Dienste oder im Englischen LocationBased Services genannt - zugeordnet werden. Dieser Abschnitt erläutert die Entstehung dieses Forschungs- und Anwendungsbereiches sowie die Anwendungskategorie Navigation, in welche die in dieser Arbeit behandelten alternativen Routen fallen. Schließlich wird klar abgegrenzt, welche Aspekte innerhalb der ortsbezogenen Anwendungen und Dienste untersucht und welche ausgeschlossen werden.

\subsubsection{Historie und Begriffserklärung}

Indirekt wurde der Grundstein für die Entwicklung von ortsbezogenen Anwendungen und Diensten gelegt, als sich die Art und Weise der Verwendung von Computern geändert hat. In den 1950er-Jahren, der Anfangszeit der elektronischen Datenverarbeitung, wurde ein Computer - damals (Groß-)Rechner oder Mainframe genannt - von mehreren Anwendern bedient und verwendet. In den 1970er-Jahren wurde der Begriff Personal Computer (PC) geprägt, wonach die raumgroßen Mainframes durch heimtaugliche Computer ersetzt wurden, die unter einem Schreibtisch Platz finden. So wurde ein Computer von einem 


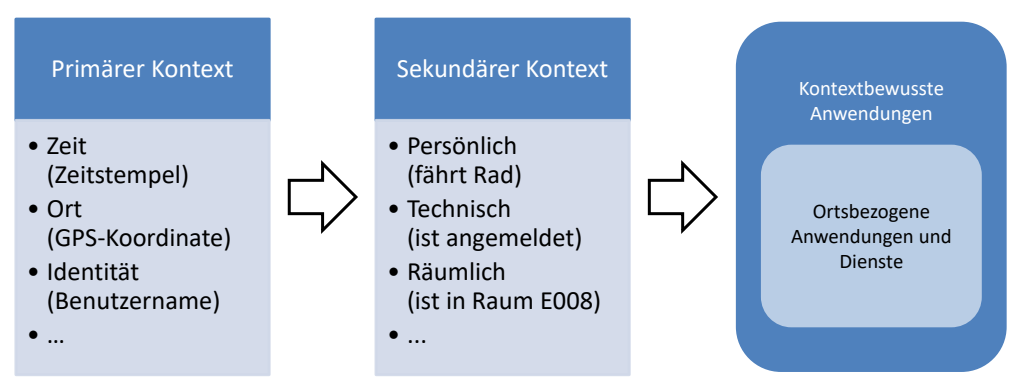

Abbildung 2.1: Zusammenhang von kontextbewussten Anwendungen und ortsbezogenen Anwendungen und Diensten. Abbildung adaptiert nach [131], Figure 1.1.

Anwender verwendet und alle Aufgaben wurden auf diesem erledigt. Seit Anfang der 1990er-Jahre wird der Begriff Ubiquitous Computing (zu Deutsch etwa Rechnerallgegenwart) verwendet, womit der kontinuierliche Rückzug der Präsenz von Computern, nicht aber deren Leistung, gemeint ist. Ein Anwender verwendet mit möglichst wenig direkter Interaktion eine Vielzahl von Computern, die durch ihre geringe Größe versteckt werden können und die in der Lage sind, miteinander zu kommunizieren. Die konsequente Weiterentwicklung der Idee zahlreicher kleiner Computer, die miteinander kommunizieren, ist das sogenannte Internet der Dinge (auch Internet of Things (IoT) genannt).

Mit der allgegenwärtigen Verfügbarkeit von Rechenleistung hat sich eine Mobilität der Computer sowie die Fähigkeit, die Umwelt mittels zahlreicher eingebauter Sensoren zu erfassen, ergeben. So ist beispielsweise ein modernes Mobiltelefon in der Lage den aktuellen Standort zu ermitteln (GPS), Videound Audiodaten aufzunehmen (Kamera und Mikrofon), die Umwelt zu messen (Barometer und Photodetektor) oder die Bewegung aufzuzeichnen (Accelerometer und Gyroskop). Ab der Jahrtausendwende hat sich ein Mobile Computing Paradigma namens Context-Aware Computing (kontextabhängige Berechnung) geformt, was besagt, dass Anwendungen Kontextinformationen wie etwa den Benutzerstandort, die Tageszeit, sich in der Nähe befindende Personen und Geräte, oder Aktivitäten erkennen und sich zunutze machen können [28]. Der Begriff der Kontextinformationen geht auf die klassische Definition von Kontext nach Dey und Abowd zurück, womit jegliche Informationen gemeint sind, die dazu verwendet werden können, die Situation einer Entität zu charakterisieren [53].

Ortsbezogene Anwendungen und Dienste sind schließlich Teil von besagtem Paradigma der kontextabhängigen Berechnung. Ein im direkten Umfeld des Anwenders getragener Computer kann mittels Sensoren beispielsweise den Kontext aktueller Standort messen, der wiederum mit anderen Informationen angereichert und in einen räumlichen Kontext wie etwa aktueller Raum überführt werden kann.

Wie in Abbildung 2.1 dargestellt adressiert die vorliegende Arbeit also orts- 
bezogene Anwendungen und Dienste, die durch die Verarbeitung von räumlichen Informationen eine Teilmenge von kontextbewussten Anwendungen darstellen.

\subsubsection{Anwendungskategorien}

Ortsbezogene Anwendungen und Dienste stellen einem mobilen Anwender Aktionen zur Verfügung, die stets einen räumlichen Bezug besitzen. Darunter fallen solch grundlegende Aktionen wie etwa Orientierung (,wo befinde ich mich?"), Suche („wo befindet sich das nächstgelegene Restaurant?"), Identifizierung (,welches Gebäude steht vor mir?"), Ereignisprüfung („was findet hier gerade statt?") und Navigation (,wie ist der Weg zu meiner Zieladresse?") [156]. Im Grunde lassen sich alle ortsbezogenen Anwendungen und Dienste auf eine Kombination oder Erweiterung dieser grundlegenden Aktionen mit räumlichem Bezug reduzieren.

Aus der Sicht des Anwendungsfalls lassen sich viele unterschiedliche Kategorien von ortsbezogenen Anwendungen und Diensten ermitteln, die unterschiedliche Anforderungen besitzen. Beispiele für Anforderungen sind Genauigkeit der Positionierung (Dezimeter oder mehrere Hundert Meter), Umgebung der Nutzung (innerhalb von Gebäuden, in Straßennetzen oder im maritimen Bereich), sowie Art der Informationsübertragung (ständige Konnektivität zwischen Endgerät und Dienstanbieter oder geräteinterne Berechnung) [169]. Eine sehr naheliegende Kategorie für Anwendungsfälle sind Notdienste. So existiert in den USA mit dem Mandat Enhanced 911 die gesetzliche Vorgabe, dass beim Absetzen eines Notrufes der geografische Standort des Anrufers automatisch übermittelt wird [183]. In die Kategorie der Informationsdienste fallen Anwendungen wie mobile Branchenbücher, bei denen Wissen über sich in der Nähe befindende Einrichtungen angezeigt wird. Im Bereich der Werbung kann dem Anwender Reklame mit Ortsbezug angezeigt oder Aktionen angeboten werden, wenn sich der Anwender in unmittelbarer Nähe des Geschäftes befindet. Die Verwendung einer Standortverfolgung und Verwaltung (auch Tracking genannt) ist beispielsweise für Kinder oder Menschen mit Demenz denkbar, aber auch für Güter in der Logistik oder im Gebäudemanagement. Ein weiterer Anwendungsfall ist die Rechnungserstellung mit Ortsbezug, wie etwa die Maut für gebührenpflichtige Straßen. In die Kategorie Spiele und Freizeit fallen das in der vergangenen Zeit überaus erfolgreiche Geocaching, eine moderne Version der Schnitzeljagd, Augmented RealitySpiele wie etwa Pokémon Go oder Ingress sowie sogenannte Buddy-Finder, die Nachrichten absetzen, sobald ein Freund räumlich nah ist. Schließlich ist eine stark ausgeprägte Kategorie von ortsbezogenen Anwendungen und Diensten die Navigation. Hierzu zählt die allgemeine Wegfindung innerhalb und außerhalb von Gebäuden, das Anzeigen von Wegbeschreibungen für gefundene Wege, das Anfragen von beispielsweise dem nächstgelegenen freien Parkplatz oder die Verwaltung von Fahrzeugflotten. 
Zusammengefasst kann gesagt werden, dass Navigation und Routenplanung ein wesentlicher Bestandteil von ortsbezogenen Anwendungen und Diensten sind, welche zwar auf eine lange Historie zurückblicken, jedoch durch den Aufschwung von leistungsfähigen Smartphones, dem Internet der Dinge, Augmented Reality sowie künstlicher Intelligenz komplett neuartige Nutzungsmöglichkeiten ermöglichen.

\subsubsection{Abgrenzung}

Der Betrieb einer konkreten ortsbezogenen Anwendung erfordert die Verwendung von vier infrastrukturellen Komponenten: das mobile Endgerät, das Kommunikationsnetz, die Positionsbestimmung und den Service- und Inhaltsanbieter [169].

Das mobile Endgerät dient einem Anwender als Werkzeug, um den ortsbezogenen Dienst zu verwenden. Beispiele für solche Geräte sind Smartphones und Laptops, aber auch in Fahrzeugen eingebaute Navigationsgeräte oder Infotainmentsysteme. Ein Entwickler einer ortsbezogenen Anwendung kümmert sich um eine geeignete Darstellung der Berechnungsrückgaben und die grafische Benutzeroberfläche, beziehungsweise die Mensch-Maschine-Schnittstelle im Allgemeinen. Die Implementierungen der in dieser Arbeit vorgestellten Ansätze und Ideen können mittels einer Konfigurationsdatei angepasst werden und besitzen eine grafische Ausgabe, die jedoch nicht auf ein spezielles mobiles Endgerät ausgerichtet ist.

Das Kommunikationsnetz ist dafür zuständig, die Anfragen des Anwenders vom mobilen Endgerät an die ortsbezogene Anwendung und deren Antwort zurück zu übertragen. Ein Entwickler muss sich entsprechend um Aspekte der verteilten Systeme kümmern, also mehrere interagierende Prozesse über Nachrichten miteinander kommunizieren lassen [35]. Da die Anwendungslogik der in dieser Arbeit vorgestellten Ansätze und Ideen im Vordergrund stehen, werden die Algorithmen nicht verteilt implementiert, wodurch der dezentrale Aspekt separiert und das Kommunikationsnetz außer Acht gelassen wird.

Die Positionsbestimmung ist eine obligatorische Komponente für eine ortsbezogene Anwendung. Neben der umfänglich eingesetzten Technik der Trilateration, wie sie beispielsweise vom Satellitennavigationssystem Global Positioning System (GPS) eingesetzt wird, existieren unterschiedliche FingerprintVerfahren. Hierbei werden im Vorfeld aufgezeichnete und entsprechend verortete Signalstärken ausgewertet [135]. Im Zuge dieser Arbeit wird stets angenommen, dass die Position des Anfragenden entweder bekannt ist oder durch manuelle Eingabe zur Verfügung gestellt wird.

Schließlich existiert die Komponente Service- und Inhaltsanbieter, die zwei grundlegende Aufgaben kombiniert. Zum einen ist diese Komponente zuständig für die Diensterbringung. Das heißt, die Anfragen des Anwenders werden von der Anwendungslogik empfangen und verarbeitet, um dann die entsprechenden Antworten zurückgeben zu können. Zum anderen kann die An- 
wendungslogik auf Inhalten basieren, die gegebenenfalls von Datenbanken einer dritten Partei bezogen werden. Die vorliegende Arbeit stellt Ansätze und Ideen vor, die von einem Service- und Inhaltsanbieter verwendet werden können.

Die soeben angesprochenen Datenbanken können entweder von Anbietern stammen, die Informationen mit Ortsbezug wie etwa Standorte von beispielsweise Geldautomaten zur Verfügung stellen oder aber von Kartierungsagenturen, die geografische Informationsmodelle anbieten. Es gibt unterschiedliche Arten von geografischen Informationsmodellen [156]. Beginnend mit der Realität und einem oder mehreren Geoobjekten, das heißt, tatsächlich existierenden und referenzierbaren Objekten, wird eine Datenbank aufgebaut, die eine möglichst exakte und vollständige geometrische Beschreibung der Geoobjekte enthält. Solche Datenbanken sind im Allgemeinen Teil von Geoinformationssystemen (GIS), die zur Verarbeitung räumlicher Daten dienen. Basierend auf diesem primären Modell können Ableitungen zu einem bestimmten Zweck entstehen. Ein Beispiel für ein solches sekundäres Modell ist ein Stadtplan oder eine Straßenkarte. Schließlich ist eine weitere Ableitung möglich, indem erstellte Karten durch Analyse und Interpretation weiter verwendet werden. Die Ansätze und Ideen der vorliegenden Arbeit knüpfen an exakt dieser Stelle der tertiären Modelle an.

Zusammengefasst werden in dieser Arbeit Ansätze und Ideen vorgestellt, die von der infrastrukturellen Komponente der Service- und Inhaltsanbieter verwendet werden können, um ortsbezogene Anwendungen und Dienste zu erstellen. Hierzu werden bereits existierende Karten verwendet, sodass durch Analyse und Interpretation neues Wissen generiert wird.

\subsection{Alternative Routen}

Das Thema der alternativen Routen ist aus Sicht der Informatik eine Teilmenge des Forschungsbereiches der Routenplanung in Verkehrsnetzen, also eine recht junge Ausprägung einer sehr alten Fragestellung. Im Bereich der Algorithmen und Datenstrukturen wird die Frage nach einer oder mehreren Routen zwischen zwei Punkten abgebildet auf die Suche nach dem kürzesten Pfad in einem Graphen. In diesem Abschnitt werden zuerst entsprechende Begriffsdefinitionen für diese Abbildung gegeben. Anschließend werden ausgewählte Lösungen für das Kürzeste-Pfad-Problem vorgestellt sowie weiterführende Problemstellungen in diesem Umfeld skizziert. Eine dieser erweiterten Problemstellungen ist die Frage nach alternativen Routen.

\subsubsection{Begriffsdefinitionen}

Gegeben sei ein gerichteter Graph $G=(V, A)$ mit einer Menge von Knoten $V$ und einer Menge von Kanten $A$. Jede Kante $e=(u, v) \in A$ ist durch zwei Knoten $u, v \in V$ definiert und besitzt eine nicht-negative Länge $l(u, v)$. Die 
Länge eines Pfades ist definiert als die Summe der Längen der involvierten Kanten.

Beim Punkt-zu-Punkt-Kürzester-Pfad-Problem sind ein Graph $G$, ein Startknoten $s \in V$ sowie ein Zielknoten $t \in V$ gegeben und die Aufgabe ist die Berechnung der Länge des kürzesten Pfades von $s$ nach $t$ in $G$. Dieser Wert heißt Distanz zwischen $s$ und $t$ und wird mit dist $(s, t)$ bezeichnet. Neben dem Punkt-zu-Punkt-Kürzester-Pfad-Problem gibt es noch Erweiterungen, sodass anstatt jeweils eines Start- und Zielknotens auch Mengen von Knoten verwendet werden können. Diese Probleme werden entsprechend one-to-all problem, all-to-one problem oder many-to-many problem genannt. Wenn die Länge des kürzesten Pfades von jedem Knoten des Graphen zu jedem anderen berechnet werden soll, so wird vom all-pairs-shortest-path problem gesprochen.

\subsubsection{Lösungen für das Kürzester-Pfad-Problem}

Das Kürzester-Pfad-Problem ist seit vielen Jahren ein beliebter Gegenstand der Forschung. Die grundlegenden Techniken wurden um 1960 entwickelt. Der wohl bekannteste Algorithmus in diesem Umfeld und zeitgleich die Standardlösung für das one-to-all problem ist der Algorithmus von Dijkstra [56]. Dieser verwendet eine Prioritätenliste $Q$, in der alle Knoten des Graphen $V \in G$ enthalten sind. Die Sortierung entspricht dabei der (vorläufigen) Distanz zum Knoten $s$. In jeder Iteration wird der Knoten mit der kleinsten Distanz entnommen und alle ausgehenden Kanten werden mitsamt deren Länge überprüft. Der Algorithmus stoppt, wenn der gesuchte Knoten $t$ überprüft wird. Die Laufzeit des Algorithmus von Dijkstra hängt somit von der gewählten Implementierung der Prioritätenliste ab, wobei hier unterschiedliche Datenstrukturen wie etwa ein binärer Heap [33] oder ein Fibonacci-Heap [82] in Frage kommen.

Die Menge der überprüften Knoten $S \subseteq V$ wird Suchraum genannt und kann beim klassischen Dijkstra-Algorithmus schnell wachsen. Eine Möglichkeit zur Reduktion der Größe des Suchraums ist die Verwendung der bidirektionalen Suche [142], wobei gleichzeitig eine Vorwärtssuche von $s$ nach $t$ und eine Rückwärtssuche von $t$ nach $s$ stattfinden. Der Algorithmus stoppt, wenn sich die beiden Suchräume treffen, das heißt, wenn ein Knoten $x$ gefunden wird, der auf dem kürzesten Pfad von $s$ nach $t$ liegt.

Hervorzuheben ist darüber hinaus der Floyd-Warshall-Algorithmus [78], der die Distanzen zwischen allen Paaren von Knoten berechnet (all-pairs-shortestpath problem). Dies kann bei dichten Graphen schneller zum Ziel führen als der entsprechend oft ausgeführte klassische Dijkstra-Algorithmus.

Neben den grundlegenden Techniken existieren zahlreiche Erweiterungen, die versuchen, den Suchraum zu verkleinern und somit Beschleunigungen der Ausführung herbeizuführen. Während der klassische Algorithmus von Dijkstra alle Knoten mit einer Distanz kleiner $\operatorname{dist}(s, t)$ überprüft, was sich als eine Art Kreis vorgestellt werden kann, versuchen sogenannte zielgerichtete Techniken Knoten auszulassen, die nicht in der Richtung von $t$ liegen. Hierzu werden 
entweder die geometrische Einbettung des Graphen, gegeben durch das Straßennetz, zu Nutze gemacht oder spezielle Eigenschaften des Graphen selbst.

Die wohl bekannteste Erweiterung ist der A*-Algorithmus [99], der eine Potentialfunktion $\pi: V \rightarrow \mathbb{R}$ verwendet, die eine untere Schranke bezüglich der Distanz $\operatorname{dist}(u, t)$ von Knoten $u$ nach Knoten $t$ darstellt. Im Grunde ist dies eine modifizierte Version des Dijkstra-Algorithmus, bei der die Prioritätenliste $Q$ durch $\operatorname{dist}(s, u)+\pi(u)$ sortiert ist. Dadurch werden Knoten, die näher am Ziel liegen, früher überprüft. Wenn die Potentialfunktion $\pi$ fehlerfrei wäre, so würden nur die Knoten des kürzesten Pfades selbst überprüft werden. Es besteht die Notwendigkeit, dass die Potentialfunktion die tatsächliche Distanz unterschätzt. Im Anwendungsbereich von Straßennetzen wird deshalb oft die geografische Distanz verwendet [11].

Ein weiterer, vielfach eingesetzter Algorithmus, ALT (A*, Landmarks, and Triangle Inequality), verwendet den A*-Algorithmus, Landmarken und die Dreiecksungleichung [94]. Er zeichnet sich durch eine verbesserte untere Schranke bei gleichzeitig garantierter Korrektheit aus. Während einer Vorverarbeitungsphase wird eine kleine Menge $L \subseteq V$ sogenannter Landmarken ausgewählt und die Distanzen werden zwischen diesen und allen Knoten im Graphen berechnet. Bei einer Kürzester-Pfad-Anfrage wird die Dreiecksungleichung auf die Landmarken angewendet, um eine valide untere Schranke für die Distanz dist $(u, t)$ für jeden Knoten $u$ zu berechnen. Die Qualität der Ergebnisse von ALT hängt von einer geeigneten Wahl der Landmarken ab, wobei in Straßennetzen weit voneinander entfernte Knoten geeignet sind.

Weitere zielgerichtete Ansätze reichern Kanten mit Informationen an, welche Knoten beginnend mit dieser Kante auf einem kürzesten Pfad erreicht werden können (geometric containers [177] und arc flags [106]), separieren den Graphen in mehrere Zellen (separator-based techniques [49]), nutzen inhärente Hierarchien des Straßennetzes aus (hierarchical techniques [91]) oder fügen virtuelle Abkürzungen ein (bounded-hop techniques [7]). Schließlich sind Kombinationen der Erweiterungen möglich, da viele der Techniken sich unterschiedliche Eigenschaften des Graphen zu Nutze machen, was entsprechende Beschleunigungen ermöglicht.

\subsubsection{Erweiterte Problemstellungen}

Es existieren viele Erweiterungen, die auf der Problemstellung nach der $\mathrm{Su}-$ che des kürzesten Pfades aufbauen. Eine solche erweiterte Problemstellung ist beispielsweise die Frage nach dem kürzesten Pfad selbst. Die oben genannten Verfahren wie etwa der Algorithmus von Dijkstra oder der A*-Algorithmus liefern grundsätzlich nur die Länge des kürzesten Pfades. Wenn der Algorithmus keine virtuellen Abkürzungen verwendet, so kann für jeden besuchten Knoten der entsprechende Vorgängerknoten vermerkt und somit vom Zielknoten über die jeweiligen Vorgängerknoten bis zum Startknoten durchlaufen werden. Verwendet ein Algorithmus virtuelle Abkürzungen, so müssen diese entpackt 
werden. Darüber hinaus gibt es die Problemstellung der stapelweisen Berechnung von kürzesten Pfaden. Dabei sollen beispielsweise alle möglichen Pfade zwischen zwei Knoten berechnet werden, was unter anderem bei Verkehrssimulationen oder der Vorhersage von Routen hilfreich ist. Schließlich besitzt das Straßennetz eine gewisse Dynamik, sodass verwendete Algorithmen auf die aktuelle Verkehrslage oder Straßensperrungen reagieren müssen.

Neben der Dynamik ist die Zeitabhängigkeit im Straßennetz ein interessanter Gegenstand der Forschung, da die Qualität einer Route oft auch von dem Zeitpunkt des Reiseantritts abhängt. Mögliche Fragestellungen sind hier die frühestmögliche Ankunftszeit, die letztmögliche Abfahrtszeit oder die Suche nach der Abfahrtszeit, die zu einer minimalen Transitzeit führt. Ferner ist es möglich, zusätzlich zur Reisezeit weitere, auch nicht-triviale Kostenfunktionen wie etwa den landschaftlichen Wert einer Strecke oder Restriktionen wie Höhen- oder Gewichtsgrenzen von Straßen oder Brücken einzusetzen. Eine weitere Herausforderung, die im Kontext der modernen Fahrradverleihsysteme (Bike-Sharing) sowie Elektrofahrzeuge auftritt, ist die Erstellung von Routen, die den Energiekonsum minimieren, möglichst bergab verlaufen oder wenig an Hauptstraßen entlang führen.

Insgesamt lässt sich beobachten, dass sich der Fokus innerhalb des traditionellen Forschungsbereichs der Routenplanung in den letzten Jahren gewandelt hat. Neben der anfänglichen Konzentration auf die Beschleunigung der Ausführung ist nun auch die moderne Verwendung des Verkehrsnetzes in den Vordergrund gerückt. Dabei werden nicht nur das Straßennetz, sondern auch fahrplanbasierte Transportnetze von Bus oder Bahn und insbesondere multimodale Szenarien betrachtet, bei denen beide Modi (straßennetz- und fahrplanbasiert) kombiniert werden. Dieser Trend wird durch die Popularität unterschiedlicher Verleihsysteme für Fahrräder und Autos sowie Online-Vermittlungsdienste für private Fahrer wie etwa Uber verschärft. Die Frage nach alternativen Routen ist somit hochaktuell, multimodale Routingalgorithmen müssen Kombinationen von Verkehrsmitteln berücksichtigen und Vermittlungsdienste für Fahrten entsprechend kalkulieren, ab wann sich das Mitnehmen einer Person lohnt.

\subsubsection{Alternative Routen in der Anwendung}

Die Erforschung von alternativen Routen in Straßennetzen ist derart fortgeschritten, dass deren Ergebnisse Einzug in kommerzielle Produkte gefunden haben. Die Online-Kartendienste Google Maps der Firma Google oder Bing Maps der Firma Microsoft, um nur zwei Beispiele zu nennen, geben bei der Routenplanung standardmäßig mehrere alternative Routen zurück. In der Literatur wird bei dem Begriff der alternativen Routen informell von angemessenen, beziehungsweise zulässigen Pfaden gesprochen, die zusätzlich zum kürzesten Pfad berechnet und präsentiert werden [2], [10]. Eine vorläufige Umschreibung solcher angemessenen, beziehungsweise zulässigen alternativen Routen, die in den Abschnitten 3.4 und 3.5 weiter konkretisiert und ausführlicher behandelt 
werden, lautet: Eine alternative Route sollte nicht viel länger als die Referenzroute sein, sollte sich substantiell von dieser unterscheiden sowie eine natürliche Form, das heißt keine unnötigen Umwege, besitzen. Wenn mehrere alternative Routen aggregiert dargestellt werden, so wird von einem Alternativgraphen gesprochen.

Sogenannte Korridore von Pfaden sind eine Problemstellung, die mit der Berechnung von alternativen Routen in Verbindung steht. Hierbei geht es darum, das Abweichen von der berechneten Route während des Fahrens zu ermöglichen, ohne dass der gesamte Pfad neu kalkuliert werden muss. Solche Routen finden Verwendung in mobilen Szenarien mit eingeschränkter Kommunikation.

Was im Zuge dieser Arbeit jedoch nicht als alternative Route angesehen wird, ist die Berechnung und Präsentation von beispielsweise der kürzesten Route, der schnellsten Route sowie der Route mit dem geringsten $\mathrm{CO}_{2}$-Verbrauch zwischen zwei Punkten. Alle drei Routen wären nämlich der kürzeste Pfad zwischen den gegebenen Punkten, nur mit unterschiedlichen Kostenfunktionen zur Berechnung der Kantenlängen.

Insgesamt kann gesagt werden, dass durch das Berechnen und Präsentieren von mehreren alternativen Routen eine personalisierte und kontextbezogene Wegefindung in Straßennetzen ermöglicht wird. Dem Anwender wird eine Auswahl zur Verfügung gestellt, sodass dieser nach eigenen Präferenzen und basierend auf persönlichen Empfindungen wählen kann.

\subsection{Komplexe Umgebungen}

Der vorige Abschnitt hat sich mit dem ersten Teil des Titels der vorliegenden Arbeit befasst, nämlich mit alternativen Routen. Das Ziel dieses Abschnittes ist es zu erläutern, was komplexe Umgebungen sind und was der grundsätzliche Unterschied zu Straßennetzen ist. Hierzu werden komplexe Umgebungen definiert sowie Kartendarstellungen als Möglichkeit der Repräsentation komplexer Umgebungen und somit der realen Welt erläutert. Anschließend werden Beispiele für ortsbezogene Dienste in komplexen Umgebungen beschrieben.

Dieser Abschnitt soll ein Verständnis dafür vermitteln, dass die Frage nach alternativen Routen auch außerhalb von Straßennetzen relevant ist.

\subsubsection{Definition komplexer Umgebungen}

Komplexe Umgebungen sind im Kontext dieser Arbeit geografische Räume, in denen eine hohe Bewegungsfreiheit herrscht. Das ist beispielsweise in Straßennetzen nicht der Fall, weswegen die Eigenschaften komplexer Umgebungen kurz beschrieben werden, um somit eine Abgrenzung und Definition zu erhalten.

Nicht ohne Grund wird die Routenplanung in einem Straßennetz auf eine Kürzeste-Pfad-Suche in einem Graphen abgebildet. Straßennetze formen eine recht natürliche Graphstruktur, da Straßen als Sequenz linearer Segmente - also Kanten - und Möglichkeiten zum Wechseln einer Straße wie etwa 
Kreuzungen als Knoten dargestellt werden können. Tatsächlich formt so das Straßennetz einen geometrischen Graphen, dessen Knoten und Kanten mit realen geometrischen Objekten verknüpft sind: Straßen, Kreuzungen und weitere bauliche Elemente sind bestimmten Orten zugeordnet. Dies führt im Ergebnis zu einer hochqualitativen Abstraktion der realen Bewegungsmöglichkeiten für einen Verkehrsteilnehmer im Straßennetz.

Anders verhält es sich jedoch in geografischen Räumen, die die vorliegende Arbeit als komplexe Umgebung umschreibt. Ein anderer Ausdruck, der in den englischsprachigen Vorveröffentlichungen der Arbeit verwendet wurde und stark im Forschungsbereich der Bewegungsplanung innerhalb der Robotik Anwendung findet, ist constrained free space, also eine eingeschränkte Freifläche. Der erste fundamentale Unterschied ist die Tatsache, dass grundsätzlich Bewegungen in alle Richtungen der Ebene möglich sind. Während in einem Straßennetz nur der Straße gefolgt und an Kreuzungen, Abbiegungen oder anderweitigen Schnittpunkten mehrerer Straßen abgebogen werden kann, ist an nahezu jeder Stelle einer komplexen Umgebung eine Bewegungsentscheidung in nahezu jede Richtung möglich. Die Freifläche wird lediglich von Hindernissen wie etwa Wänden oder Möbeln limitiert, weshalb von eingeschränkten Freiflächen die Rede ist.

Zum Teil können Konzepte vom Straßennetz auf komplexe Umgebungen übertragen werden, teilweise müssen aber auch neue eingeführt werden. Ein Konzept, was in Straßennetzen nicht vorkommt, sind mehrere Stockwerke innerhalb eines Gebäudes. Die eigentliche Existenz und insbesondere die Verknüpfung der Stockwerke über Aufzüge oder Treppenhäuser müssen abgebildet werden. Für gewöhnlich werden mehrere Stockwerke nicht über eine $3 D$-Darstellung abgebildet, sondern über ein 2,5D-Datenmodell: es existieren mehrere, gewöhnliche 2D-Darstellungen, die an bestimmten Stellen (beispielsweise Aufzug oder Treppenhaus) miteinander verbunden sind. Ein Konzept, das in komplexen Umgebungen zunächst nicht vorhanden ist, ist die inhärente Hierarchie von Straßennetzen. Basierend auf Entwurfsvorschriften existieren in Deutschland und anderen Ländern Straßenkategorien wie etwa Autobahnen oder Landstraßen.

Zusammengefasst ist im Kontext der vorliegenden Arbeit mit komplexer Umgebung ein geografischer Raum gemeint, der als eine oder mehrere zweidimensionale Flächen dargestellt wird, in dem beliebige Bewegungen durchgeführt werden können, die nur von Hindernissen wie etwa Wänden oder Möbeln eingeschränkt sind. Dabei ist es unerheblich, ob es beispielsweise ein Gebäudeplan mit ausschließlich überdachten Räumen oder auch Bereichen unter freiem Himmel ist, oder aber eine Parklandschaft, deren Freifläche durch Teiche oder Hecken beschränkt ist. Neben der erhöhten Bewegungsfreiheit herrscht in komplexen Umgebungen außerdem eine hohe Mehrdeutigkeit in Bezug auf die unterschiedlichen Verwendungsmöglichkeiten des Freiraums und der dadurch entstehenden Semantiken. Die Kernfrage ist daher, ob und wie Forschungsergebnisse aus dem Bereich der Straßennetze innerhalb von komplexen Um- 
gebungen und deren unterschiedlichen Kartendarstellungen eingesetzt werden können.

\subsubsection{Kartendarstellungen}

Kartendarstellungen von real existierenden oder virtuellen geografischen Räumen werden unter anderem im Bereich der Routenplanung dafür verwendet, den Suchraum, der die möglichen Bewegungen beinhaltet, zu reduzieren. Damit wird zum einen eine effizientere Ausführung der Algorithmen ermöglicht, zum anderen wird dadurch aber gleichzeitig die Fähigkeit eingeschränkt, jeden möglichen Pfad innerhalb des geografischen Raums zu beschreiben. Es existieren viele unterschiedliche Kategorien von Kartendarstellungen, wobei die wichtigsten Kategorien in diesem Abschnitt kurz erläutert werden.

Bei sogenannten constrained grids (etwa beschränkte Gitter) wird eine grundsätzliche Kantenlänge definiert und damit ein strukturiertes Gitter fester Kantenlänge erstellt. Dieses Gitter, bestehend aus Gitterzellen, Gitterpunkten und Gitterlinien, wird anschließend über den gegebenen geografischen Raum gelegt und partitioniert diesen lückenlos und ohne Überlappung. Für jeden Gitterpunkt wird ein Knoten im Graphen erstellt, und die Knoten zweier benachbarter Gitterpunkte sind durch eine Kante verbunden, wenn die entsprechende Gitterlinie ausschließlich durch frei begehbare Flächen verläuft. Die Kantenerstellung basierend auf dem überlagerten geografischen Raum stellt die besagte Beschränkung dar. Belegungsraster sind eine Teilmenge von constrained grids und finden starke Verwendung in der Routenplanung von mobilen Robotern, aber auch bei der generellen Ablaufplanung von diskreten Konfigurationen. Belegungsraster können als zweidimensionale Rastergrafiken (Bitmaps) dargestellt werden, die frei begehbare Flächen in einer Farbe ausdrücken (beispielsweise weiß) und Hindernisse in einer anderen Farbe (etwa schwarz). Anschließend wird für jeden Bildpunkt (Pixel) ein Knoten im Graphen eingefügt. Zwischen zwei Knoten existiert eine Kante, wenn die entsprechenden Bildpunkte beide weiß und direkte Nachbarn sind. Das bedeutet, dass eine Bewegung von dem einen zum anderen Bildpunkt möglich ist. Der wohl größte Nachteil von constrained grids ist die Tatsache, dass je nach gewählter Kantenlänge das Gitter groß und ineffizient werden kann. Ausgedehnte Freiflächen werden durch viele Knoten und Kanten dargestellt, bei ungünstig gewählter Zellengröße und -lage können aber auch feine Strukturen eines Gebäudes nicht bemerkt werden. Trotzdem besitzen constrained grids wichtige Eigenschaften, die für eine Verwendung in der Routenplanung sprechen. Zum einen sind aktuelle Computer - beispielsweise durch Vektorisierung und etwaige Prozessorerweiterungen - in der Lage, große Rastergrafiken effizient zu verarbeiten. Zum anderen besitzen mittels constrained grids erstellte Graphen einen limitierten Knotengrad von 4, wenn nur vertikal oder horizontal benachbarte Knoten verbunden werden, oder einen Knotengrad von 8, wenn zusätzlich die Diagonalen einbezogen werden. Schließlich hängt die Leistung der Algorithmen, die den Graphen verwenden, 
indirekt nur von der Menge der frei begehbaren Fläche ab und nicht von der beteiligten Geometrie: durch das beschränkte Gitter und dessen hohe Abstraktionskraft ist es somit unerheblich, ob ein Hindernis fein strukturiert ist oder von simpler Form.

Eine weitere Art von Kartendarstellungen sind polygonal maps (etwa polygonale Karten), bei denen Hindernisse wie beispielsweise Wände oder Gegenstände als Polygone modelliert werden. Die Ecken und Seiten der Polygone werden zur Grapherstellung verwendet, wobei es unterschiedliche Alternativen der Konstruktion gibt. Eine Möglichkeit sind sogenannte Sichtbarkeitsgraphen (visibility graphs), bei denen alle Ecken der Polygone verbunden werden, wenn die direkte Sichtlinie ausschließlich durch frei begehbare Flächen verläuft. Der größte Vorteil von Sichtbarkeitsgraphen ist die Tatsache, dass alle kürzesten Pfade zwischen den Ecken der Hindernispolygone im Graphen enthalten sind. Dieser Vorteil kann jedoch auch zum Nachteil werden, da die berechneten kürzesten Pfade recht unnatürlich aussehen, parallel zu Wänden laufen oder in Diagonalen durch größere Freiflächen verlaufen. Ein weiterer Vorteil ist, dass wenn der abzubildende geografische Raum aus wenigen und simplen Hindernissen besteht, so ist auch der resultierende Graph klein. Der Knotengrad des Graphen kann jedoch schnell stark ansteigen, wenn das Umgebungsmodell detailreiche Objekte enthält, wie beispielsweise eine runde Säule, die durch viele Liniensegmente angenähert wird. Schließlich ist zusätzlicher Aufwand notwendig, wenn bei einer Routenplanung der Start- oder Zielpunkt nicht auf einer Polygonkante liegt: es muss entschieden werden, welcher benachbarte Knoten im Graphen stattdessen verwendet wird.

Unter navigation meshes (etwa Navigationsnetze) werden Karten verstanden, bei denen nicht die Hindernisse, sondern die frei begehbaren Flächen durch Polygone dargestellt werden. In vielen Fällen werden für diese Tesselation, also die lückenlose und überlappungsfreie Partitionierung des Freiraums, Dreiecke verwendet. Um aus dem navigation mesh einen Graphen zu erstellen, wird für jedes Polygon ein Knoten erstellt. Eine Kante wird in den Graphen eingefügt, wenn zwei Polygone sich eine Seite teilen, man sich also von dem einen in das andere Polygon bewegen kann. Das Verwenden von möglichst einfachen Polygonen wie Dreiecken hat den Vorteil, dass der resultierende Graph einen niedrigen Knotengrad besitzt. Gewissermaßen ist der oben erwähnte Ansatz der Belegungsraster auch ein navigation mesh, bei dem kleine Quadrate als Polygone verwendet werden. Bei der Realisierung eines Navigationsgraphen aus dem navigation mesh können verschiedene Anpassungen vorgenommen werden. Unter anderem stellt sich die Frage nach der Platzierung des Knotens, der ein Polygon repräsentiert (beispielsweise der Schwerpunkt des Polygons). Außerdem kann die Kante zwischen zwei benachbarten Polygonen derart angepasst werden, dass sie beispielsweise zuerst durch einen Hilfsknoten auf der Mitte der entsprechenden Seite verläuft. Dies kann bei gewissen räumlichen Gegebenheiten zu natürlicheren Pfadverläufen führen.

Die vorliegende Arbeit bezieht sich auf geografische Räume, die mittels Be- 


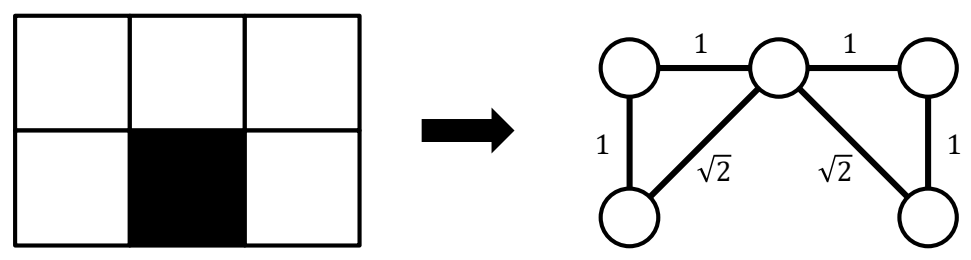

Abbildung 2.2: Beispiel für die Umwandlung eines Belegungsrasters mit $2 \times$ 3 Zellen in einen Graphen. Der isolierte Knoten, der aus der einzigen nicht begehbaren Zelle (schwarzes Pixel) resultiert, ist nicht dargestellt.

legungsrastern dargestellt werden. Die Frage nach der Route zwischen zwei gegebenen Punkten einer Karte, übergeben als $x$ - und $y$-Koordinate, wird wie auch bei der Routenplanung in Straßennetzen als Kürzester-Pfad-Problem in einem gewichteten Graphen abgebildet und gelöst. Wie oben beschrieben wird jedes Pixel als Knoten im Graphen angelegt und zwei direkt benachbarte, weiße Pixel erzeugen eine Kante im Graphen. Horizontal und vertikal benachbarte Pixel erzeugen Kanten mit einem Gewicht von 1 und diagonal benachbarte weiße Pixel entsprechend Kanten mit einem Gewicht von $\sqrt{2}$. Abbildung 2.2 zeigt ein entsprechendes Beispiel, bei dem der aus dem schwarzen Pixel resultierende Knoten ausgelassen wird.

Zusammengefasst kann gesagt werden, dass im Umfeld komplexer Umgebungen die Erstellung und Verwendung einer Kartendarstellung zwecks Routenplanung weniger eindeutig ist als in Straßennetzen. Die verschiedenen Kategorien und Implementierungen der Kartendarstellungen mit ihren jeweiligen Vor- und Nachteilen stellen stets einen Kompromiss zwischen der Komplexität der einzusetzenden Algorithmen und der Ausdruckskraft bezüglich der Repräsentation der Bewegungen dar. Vereinfachende Kartendarstellungen reduzieren die Anzahl der Knoten und Kanten des Navigationsgraphen und entsprechend die Komplexität der Algorithmen. Jedoch führt dies zu Ungenauigkeiten, da zum einen der geografische Raum simplifiziert und zum anderen die Menge der darstellbaren Bewegungen reduziert wird.

\subsubsection{Ortsbezogene Dienste in komplexen Umgebungen}

In Abschnitt 2.1.2 wurden unterschiedliche Kategorien von ortsbezogenen Anwendungen und Diensten vorgestellt. Viele von diesen Anwendungskategorien lassen sich auch in komplexen Umgebungen, also beispielsweise innerhalb von Gebäuden, einsetzen. Allerdings gibt es darüber hinaus noch weitere Anwendungen, die speziell für die Navigation von Fußgängern oder zumindest in frei begehbaren Bereichen gedacht sind.

Selbst die alleinige Positionierung einer Person innerhalb eines Gebäudes mithilfe eines Smartphones stellt einen Mehrwert dar. In großen Einkaufs- 
zentren, Flughäfen oder Messegeländen kann die eigene Verortung stark zur Orientierung beitragen. Wenn der Gedanke der Positionierung einer Person weitergeführt wird, so bietet sich zum Beispiel auch die Anwendung des Geofencings (Kunstwort, etwa geografisches Einzäunen) an. Hierbei wird eine Aktion automatisiert ausgelöst, wenn eine Person oder ein Objekt die Begrenzung der definierten Fläche betritt oder verlässt. Dies kann zur Präsentation von Informationen oder zum Angebot von speziellen Rabatten genutzt werden, wenn eine Person ein Geschäft betritt oder sich vor diesem aufhält. Wenn die Positionierung sehr exakt ist, so können auch Informationen zu einem konkreten Produkt im Ladenregal angeboten werden.

Ein weiterer Anwendungsfall ist die Unterstützung bei der Findung von freien Plätzen in Parkhäusern. Hierbei können zum Beispiel die Sendemodule für die signalstärkebasierte Positionierung in bereits existierende Leuchtstoffröhren des Parkhauses installiert werden, um Fahrzeuge und Personen innerhalb des Parkhauses zu verorten. Sensoren, die an den Decken oberhalb der Parkplätze angebracht sind, melden der Anwendung die noch freien Plätze. Schließlich kann einem Autofahrer ein freier Parkplatz sowie die Route dorthin angezeigt werden.

Neben Menschen können auch mobile Roboter Anwender von ortsbezogenen Diensten sein - oder diese selbst darstellen. Derzeit können mobile Roboter in Lagerhäusern einfache Aufgaben wie den Transport von Paletten durchführen, wofür sie die Aktionen der Positionierung und Routenplanung verwenden. Im Sinne des Entwicklungskonzeptes „Smart City“ sollen mobile Roboter bald auch näher am Menschen arbeiten, beispielsweise als Lotse für eine Reisegruppe durch einen Flughafen [174] oder als Assistenzroboter zur Alltags- und Haushaltsunterstützung [154]. Ein weiteres Einsatzfeld für mobile Roboter, die ortsbezogene Dienste verwenden oder selbst anbieten, ist die Durchführung von komplexen Aufgaben in gefährlichen Umgebungen. Ein Beispiel hierfür ist die Erkundung von zerstörten Gebäuden bei Nuklearkatastrophen wie etwa dem Kernkraftwerk in Fukushima [113], [141]. Hervorzuheben ist die DARPA Robotics Challenge, ein internationaler Robotikwettbewerb, der genau solche Fragestellungen untersucht [151].

In Bezug auf die Fußgängernavigation sind zahlreiche konkrete Anwendungsfälle denkbar. Es können für Fabrikarbeiter, die Wartungsarbeiten an mehreren Stellen in einem Industriegebäude durchführen, effiziente Laufwege erstellt werden. Besucher unbekannter Gebäude wie etwa Krankenhäuser, Flughäfen oder Messen können Unterstützung bezüglich der Orientierung oder Wegefindung erhalten. Ebenso ist ein Navigationssystem für blinde Menschen denkbar, bei dem spezielle Routen gesucht werden, die zum Beispiel gefährliche oder komplexe Kreuzungen vermeiden und entsprechende Navigationsanweisungen erstellt werden.

Ein weiterer sowohl aus wissenschaftlicher als auch wirtschaftlicher Sicht interessanter Bereich ist das Routing von Nicht-Spieler-Charakteren in Videospielen. Verschiedene Gattungen von Spielen wie etwa Ego-Shooter oder 
Echtzeit-Strategiespiele verwenden Informationen über die lokale räumliche Umgebung, um so den menschlichen Spieler mit Taktiken oder Manövern der Nicht-Spieler-Charaktere zu überraschen.

Schließlich sind gänzlich neuartige Dienste denkbar wie beispielsweise eine einfach durchzuführende 3D-Kartierung eines Gebäudes inklusive der Erstellung einer zentimetergenauen 3D-Punktwolke mitsamt Navigationsgraphen [111].

Zusammengefasst kann gesagt werden, dass das Interesse an ortsbezogenen Anwendungen und Diensten in komplexen Umgebungen sowohl in der Forschung als auch in der konkreten Benutzung stark ansteigt. Die starken Fortschritte in den Bereichen der Augmented Reality, Virtual Reality oder der Mensch-Roboter-Interaktion werden diesen Trend beschleunigen und vermutlich zu einer steten Integration der ortsbezogenen Diensten in den Alltag führen.

\subsection{Anwendungsfälle für alternative Routen in komplexen Umgebungen}

Im vorliegenden Kapitel wurden ortsbezogene Anwendungen und Dienste erläutert, der Zweck alternativer Routen in Straßennetzen beschrieben sowie Anwendungsfälle für die Routenplanung in komplexen Umgebung dargestellt. In diesem Abschnitt werden nun Anwendungsfälle für alternative Routen in komplexen Umgebungen vorgestellt, also eine Kombination der soeben aufgeführten Themenbereiche.

Bei der personalisierten Fußgängernavigation in unbekannten Gebäuden können einem Anwender nicht nur die eigene Position oder ein Weg von dem aktuellen Standort zu einem beliebigen Ziel berechnet und dargestellt, sondern unter Zuhilfenahme von zusätzlichen Kontextinformationen auch interessante Alternativen angeboten werden. So können einem Besucher eines Flughafens basierend auf dem Geschlecht, dem Zielort, der verbleibenden Wartezeit oder weiterer Präferenzen unterschiedliche Wege präsentiert werden, wie etwa eine Route mit Ausblick auf startende Flugzeuge, eine Route vorbei an hochpreisigen Geschäften oder aber eine Route, die ein bestimmtes Restaurant als Zwischenziel hat.

Mobile Roboter in Industriegebäuden erhalten alternative Routen, um beispielsweise Messungen an verschiedenen Stellen des Gebäudes durchzuführen, und in Lagerhäusern können alternative Routen Engpässe durch zu starke Verwendung derselben Hauptwege vermeiden.

In Einsatzleitstellen während der Brandbekämpfung oder im Katastrophenschutz kann die Berechnung alternativer Routen den Rettungs- und Einsatzkräften dabei helfen, blockierte Bereiche zu vermeiden. Mittelfristig ist es auch denkbar, dass solche Routen mittels Augmented Reality in das Visier des Feuerwehrhelms eingeblendet werden. 
Wie im vorigen Abschnitt angesprochen, müssen Nicht-Spieler-Charaktere von Ego-Shootern oder Echtzeit-Strategiespielen taktische Manöver in komplexen Umgebungen ausführen. Dazu zählt die intelligente Verwendung der örtlichen Begebenheiten, also beispielsweise das Begehen alternativer Routen zum Zwecke des Zangenangriffs oder Kreuzfeuers. Darüber hinaus kann einem Nicht-Spieler-Charakter eine Menge von Routen zur Auswahl gegeben werden, um so eine erschwerte Vorhersagbarkeit der Bewegungen zu erreichen.

Schließlich können alternative Routen innerhalb großer Gebäudekomplexe wie etwa Flughäfen, Messen oder Stadien dazu genutzt werden, eine proaktive Regulierung von Personenströmen vorzunehmen. Durch die an den aktuellen Zustand angepasste Öffnung von Toren und Schleusen können so Engpässe vermieden werden.

\subsection{Zusammenfassung und Ausblick}

In diesem Kapitel wurde der Forschungsbereich der ortsbezogenen Anwendungen und Dienste eingeführt. Die vorliegende Arbeit behandelt Problemstellungen der Routenplanung und Navigation, was ein Teilgebiet davon ist. Die Ideen und Ansätze, die in dieser Arbeit vorgestellt werden, sind Bausteine zur Entwicklung neuartiger ortsbezogener Dienste.

Das große Ziel der Wissenschaftsgemeinde, die sich mit der Erforschung von Routenplanung beschäftigt, kann sicherlich als weltweit verfügbares Navigationssystem umschrieben werden, das die Kombination unterschiedlicher Verkehrsmittel - also auch den Fußverkehr - berücksichtigt, aktuelle und historische Verkehrsinformationen in Echtzeit einbezieht und zudem eine personalisierte Verwendung ermöglicht [11]. Alternative Routen sind demnach inhärenter Bestandteil dieser Vision, und die übergangslose Verwendung von entsprechenden ortsbezogenen Diensten sowohl innerhalb als auch außerhalb von Gebäuden ist Gegenstand aktueller Forschung [145].

Einer der größten Unterschiede bei der Routenplanung in Straßennetzen und komplexen Umgebungen ist die Tatsache, dass für komplexe Umgebungen zahlreiche unterschiedliche Möglichkeiten zur Modellierung des Raumes denkbar sind, sprich unterschiedliche Kartendarstellungen. Nachdem in diesem Kapitel die wichtigsten Kategorien von Kartendarstellungen vorgestellt wurden, wurde erläutert, dass die vorliegende Arbeit sich auf Belegungsraster fokussiert.

Unter anderem aufgrund der rasanten Entwicklungen im Bereich der Augmented Reality - und auch der Virtual Reality - erweitert sich das Feld der Navigation und Routenplanung, sodass nicht mehr nur Navigationssysteme für Straßennetze betrachtet werden. Zukünftig werden personalisierte Assistenzsysteme eine allgegenwärtige Navigation ermöglichen, wie etwa die EchtzeitEinblendung von Richtungsangaben in Brillen mittels Augmented Reality. Die vorliegende Arbeit kann mit den vorgestellten Ideen und Ansätzen Werkzeuge dafür zur Verfügung stellen. Darüber hinaus gewinnt die Personalisierung und die facettenreiche Einbeziehung von Kontext stetig an Bedeutung, weshalb die 
Analyse der lokalen räumlichen Umgebung, also des räumlichen Kontextes, eine wichtige Forschungsfrage ist. 


\section{Berechnung alternativer Routen in Freiflächen}

Wie der Titel der vorliegenden Arbeit erkennen lässt, fokussiert sie sich auf die Berechnung und Verarbeitung alternativer Routen, jedoch nicht wie weithin bekannt in Straßennetzen, sondern in komplexen Umgebungen, was frei begehbare Szenarien wie etwa Parks oder Flughäfen sein können.

In diesem Kapitel wird zuerst eine formale Definition für alternative Routen in komplexen Umgebungen gegeben sowie eine effizient $\mathrm{zu}$ berechnende Annäherung dieser Definition (Abschnitt 3.2). Anschließend werden Algorithmen vorgestellt, mit denen solche alternative Routen berechnet werden können (Abschnitt 3.3). Sowohl die Definition als auch die Algorithmen entspringen der Veröffentlichung [179], bei der der Autor der vorliegenden Arbeit mitgewirkt hat. Zusammen stellen diese beiden Beiträge den Ausgangspunkt der vorliegenden Arbeit dar, da sie bei den folgenden Ideen und Verfahren oft zur Erstellung der Eingabedaten verwendet werden.

Nach der Definition alternativer Routen in komplexen Umgebungen sowie der Beschreibung von Algorithmen zum Finden selbiger wird gezeigt, warum die Konzepte der weit fortgeschrittenen Literatur bezüglich alternativer Routen in Straßennetzen nicht uneingeschränkt in komplexen Umgebungen verwendet werden können. Zuerst wird der Stand der Technik bezüglich der Qualität von alternativen Routen und Alternativgraphen strukturiert aufgezeigt (Abschnitt 3.4). Anschließend wird diskutiert, warum die besagten Maße nicht unverändert im Kontext komplexer Umgebungen verwendet werden können (Abschnitt 3.5). Ziel dieser Diskussion ist eine Anregung der Entwicklung von Qualitätsmetriken im Umfeld komplexer Umgebungen.

\subsection{Motivation und Herausforderungen}

Das Finden der kürzesten Routen von einem spezifischen Startpunkt zu einem Ziel ist ein altbekanntes Problem der Forschung (siehe Abschnitt 2.2.2) und kann sowohl in Straßennetzen als auch in komplexen Szenarien mittels des Algorithmus von Dijkstra oder erweiterten Versionen dieser grundlegenden Technik behandelt werden. In vielen Anwendungsfällen hat ein Anwender jedoch eine komplexere Vorstellung davon, was eine gute Route ist. Wenn beispielsweise ein Pfad innerhalb eines Gebäudes geringfügig länger als der kürzeste Pfad ist, jedoch nur eine Abbiegung statt zwei oder mehreren besitzt, so ist es wahrscheinlich, dass ein Anwender diesen Pfad präferieren wird. Ein weiteres 
Beispiel wäre ein kürzester Pfad, der das Stockwerk wechselt und somit zwei Treppenhäuser durchläuft und ein geringfügig längerer Weg, der die Etage gar nicht wechselt. Nicht nur für Menschen mit Gehbehinderung könnte dies eine vorteilhafte Wahl sein.

Zusammengefasst kann gesagt werden, dass der kürzeste Pfad zwischen zwei Punkten von einem Anwender nicht zwingend als der beste Pfad angesehen werden muss. In einer solchen Situation sollte versucht werden, eine Route zu erstellen, bei der mehr Metriken als nur die Länge des Pfades verwendet oder aber zusätzliche Einschränkungen wie etwa „möglichst keine Treppenhäuser“ berücksichtigt werden. Die resultierende Aufgabe ist damit die multikriterielle Optimierung von Routen. Im Allgemeinen ist eine solche Pareto-Optimierung schwer zu lösen [62], denn in vielen Fällen können verschiedene Pfade nicht konsistent verglichen werden. Mit anderen Worten ausgedrückt ist nicht ohne weiteres klar, ob beispielsweise 10\% kürzer besser ist als $10 \%$ schneller.

Bei der Navigation kann jedoch davon ausgegangen werden, dass eine primäre Zielfunktion definiert ist, die es zu maximieren gilt, um anschließend weitere Zielfunktionen in einem nachgelagerten Schritt zu integrieren. Das bedeutet, dass in einem ersten Schritt Routen unterschiedlicher Länge und unterschiedlicher Form berechnet werden, um diese anschließend zu filtern, sodass nur noch $k$ angemessen kurze Pfade, die ausreichend unterschiedlich zueinander sind, erhalten bleiben. Dies ist im Grunde die Vorgehensweise, wie sie aktuell bei der Erstellung von alternativen Routen und Alternativgraphen im Straßenverkehr angewendet wird [11]. Es zeigt sich, dass die Definition von angemessen kurz und ausreichend unterschiedlich nicht trivial ist. Bei der Navigation in Straßennetzen ist es gebräuchlich, ein ausreichend unterschiedlich zu erreichen, indem das wesentliche Routensegment, etwa eine lange Autobahnstrecke, durch ein anderes ausgetauscht wird. Angemessen kurz ist gegeben durch die Länge des kürzesten Weges, der eine andere Autobahn oder Landstraße verwendet.

Bei Navigationsszenarien innerhalb von komplexen Umgebungen ist jedoch die Definition von alternativen Routen schwieriger. Beispielsweise existieren weniger Abweichungen in der Reisegeschwindigkeit, da im Allgemeinen keine Geschwindigkeitsbegrenzungen für Fußgänger existieren und sich die Geschwindigkeitsskala auf wenige Kilometer pro Stunde $(\mathrm{km} / \mathrm{h})$ beschränkt und nicht wie im Straßenverkehr auf weit über $100 \mathrm{~km} / \mathrm{h}$ [134]. Zudem führt die Navigation in Freiflächen oft zu einer Vielzahl an unterschiedlichen Wegen mit gleicher Länge. Mit anderen Worten: wenn die Algorithmen zum Finden von alternativen Routen in Straßennetzen unverändert in komplexen Umgebungen wie etwa Gebäuden angewendet werden, so können potentiell viele Wege entstehen, die semantisch äquivalent sind hinsichtlich der Tatsache, dass sie dieselben Türen, Räume und Freiflächen durchqueren.

Wie bereits in Abschnitt 2.4 beschrieben ist ein hoher Nutzen in der Berechnung und Auswertung von alternativen Routen in komplexen Umgebungen dadurch gegeben, dass die Auswahl eines Pfades durch Elemente entlang des 
Pfades personalisiert werden kann. Beispielsweise kann an einem Flughafen basierend auf Kontextinformationen wie etwa verbleibende Zeit, Geschlecht oder Alter unterschiedliche Routen berechnet und diese als Alternativgraph dem Anwender präsentiert werden. Dieser kann zum Beispiel eine Route, die kurz und deshalb schnell ist, eine Route, die an bei Kindern beliebten Geschäften vorbeiführt, oder eine Route, die an vielen Restaurants entlang läuft, enthalten. Wenn nur wenige Informationen über den Anwender bekannt sind, so kann das System die Logos von Geschäften einblenden, die beim Ablaufen der Route aus sichtbar wären, damit die Person selbst auswählen kann.

Zusammengefasst lässt sich sagen, dass in diesem Kapitel der Grundstein der vorliegenden Arbeit behandelt wird. Dies bildet die Definition und Berechnung von alternativen Routen in komplexen Umgebungen, damit diese in nachfolgenden Schritten weiter verarbeitet und analysiert werden können. Außerdem sind dies Maße für die Qualität solcher alternativer Routen oder Alternativgraphen. Daher werden in diesem Kapitel vier Herausforderungen bearbeitet.

1. Die erste Herausforderung liegt in der Ermittlung einer Definition, wann zwei gegebene Pfade innerhalb einer 2D-Kartendarstellung als gleichwertig oder äquivalent gelten und wann als alternativ. Eine solche Funktion, die beliebige Routen in disjunkte Untermengen teilt (gleichwertig oder alternativ), entspricht einer Äquivalenzrelation.

2. Anschließend wird in diesem Kapitel eine effiziente Methode zur Berechnung dieser Relation vorgestellt. Sie ist eine Annäherung der Äquivalenzrelation und nutzt simplifizierende Annahmen aus, die jedoch der Praxis standhalten.

3. Die nächste Herausforderung ist das tatsächliche Berechnen solcher nichtäquivalenter Pfade. Es existieren unendlich viele Möglichkeiten, zwei Punkte durch einen Pfad zu verbinden, weshalb Heuristiken zur Berechnung von geeigneten Kandidaten gesucht sind.

4. Schließlich ist eine weitere Herausforderung dadurch gegeben, dass potentiell viele erstellte alternative Routen bewertet werden müssen, damit - je nach Anwendungslogik - nicht alle Pfade dem Anwender zurückgegeben werden. Demnach werden Auswahlkriterien für hochqualitative Alternativgraphen, also die kompakte Darstellung mehrerer alternativer Routen, behandelt.

\subsection{Definition alternativer Routen mittels Homotopie}

In Straßennetzen ist eine alternative Route - vorerst ohne qualitative Wertung - dadurch definiert, dass sie eine andere Kantenfolge in der verwendeten 
Graphdarstellung besitzt. Innerhalb von komplexen Umgebungen, bei denen sich ein Anwender frei in der Fläche bewegen kann, verhält sich dies anders. Auch wenn technisch gesehen unterschiedliche Kanten durchlaufen werden, so kann die Route als semantisch identisch angesehen werden.

In diesem Abschnitt werden demnach alternative Routen in komplexen Umgebungen definiert. Hierzu wird eine Äquivalenzrelation verwendet, die mittels des topologischen Konzepts der Homotopie erstellt wird (Abschnitt 3.2.1). Zudem wird ein Verfahren zur effizienten Annäherung dieser Relation vorgestellt (Abschnitt 3.2.2).

Es wird also auf zwei der in Abschnitt 3.1 beschriebenen Herausforderungen eingegangen: Zum einen wird definiert, wann zwei Pfade als gleichwertig oder alternativ angesehen werden können. Zum anderen wird eine effiziente Methode zur Berechnung dieser Relation vorgestellt.

\subsubsection{Homotopie als Äquivalenzrelation}

Die grundsätzliche Idee hinter der Verwendung des Konzepts der Homotopie soll mit einem Beispiel motiviert werden, das mit alternativen Routen in einem Straßennetz beginnt. Abbildung 3.1a zeigt einen Ausschnitt eines Straßennetzes sowie drei Routen mit gleichem Start und Ziel. Die links und mittig dargestellten blauen Routen können intuitiv als Alternativen zur rechts dargestellten roten Route angesehen werden, da letztere den vertikal verlaufenden Fluss an einer anderen Brücke überquert. Ob die beiden blauen Routen alternativ zueinander sind oder äquivalent ist fraglich, da sie prinzipiell den gleichen Verlauf besitzen und sich nur minimal unterscheiden. Nun wird dieses Beispiel in ein Szenario umgewandelt, in dem sich ein Anwender frei in der Fläche bewegen kann, also ohne Berücksichtigung von vorgegebenen Straßen. Dies ist in der Terminologie dieser Arbeit eine komplexe Umgebung. Zusätzlich kann ohne Beschränkung der Allgemeinheit davon ausgegangen werden, dass sich dieses Szenario innerhalb eines Gebäudes befindet. In diesem Fall ist der Fluss aus dem Straßennetz ein Hindernis wie eine Wand und die Brücken sind Durchgänge wie Türen. Wie in Abbildung 3.1b dargestellt können die zwei blauen Routen, die durch die untere „Tür“ verlaufen, als eine Möglichkeit zum Erreichen des Ziels angesehen werden und die rote Route, die entsprechend durch die obere „Tür" verläuft, als eine andere Möglichkeit.

Die Frage lautet nun, wie eine formale Definition gegeben werden kann, mit der entschieden wird, ob zwei Pfade $p$ und $q$ als äquivalent angesehen werden können oder als alternativ. Zudem ist eine effiziente Methode notwendig, um diese Relation für explizite Routen zu berechnen. Die grundsätzliche Vorgehensweise, die in [179] vorgestellt wird, ist wie folgt: zwei Routen mit gleichem Start und Ziel gelten als äquivalent, wenn sie ohne „Sprung“ oder ,Schnitt“ ineinander überführt werden können. Zwei Routen mit gleichem Start und Ziel gelten demnach als alternativ, wenn ein Hindernis zwischen ihnen liegt. Abbildung 3.2 verdeutlicht dies. Zwischen den Routen $q$ und $r$ liegt kein Hindernis, 

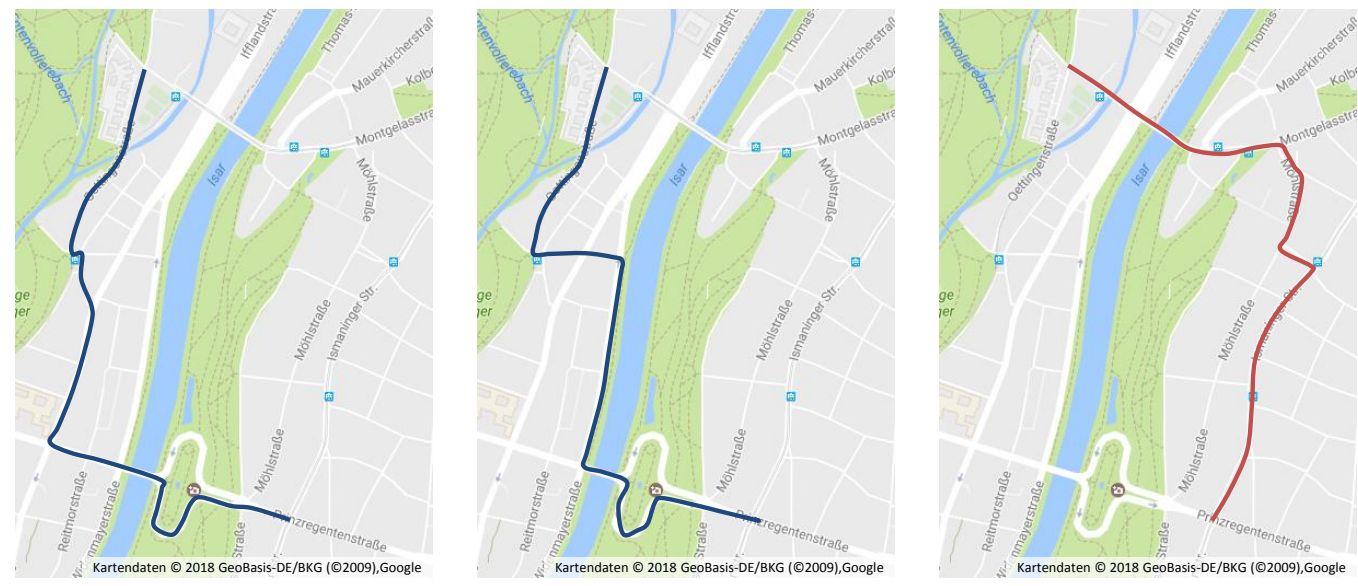

(a) Alternative Routen im Straßennetz.
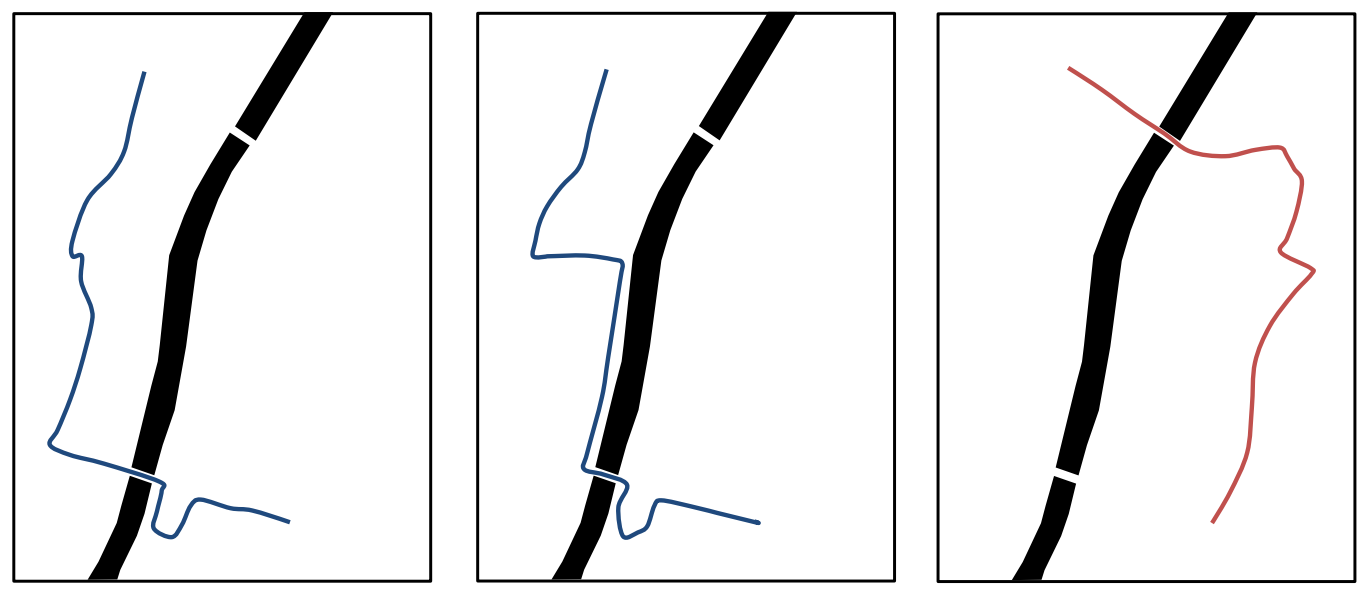

(b) Alternative Routen in komplexer Umgebung.

Abbildung 3.1: Homotopie als ein Indikator für die Äquivalenz von Routen. 


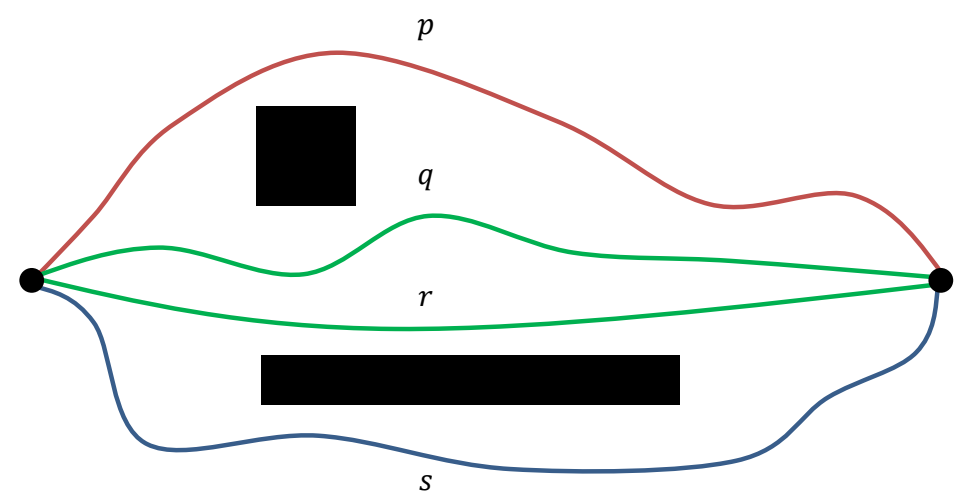

Abbildung 3.2: Verdeutlichung des Konzepts: Die Routen $q$ und $r$ können ohne ,Sprung" oder „Schnitt“ ineinander überführt werden und gelten demnach als äquivalent. Zwischen $r$ und $s$ liegt ein Hindernis, weshalb sie als alternativ angesehen werden.

sodass sie ohne Sprung oder Schnitt ineinander überführt werden können. Sie gelten als äquivalent. Die Routen $r$ und $s$ können entsprechend als alternativ angesehen werden.

Zur formalen Definition dieser Idee wird die Verwendung der Homotopie vorgeschlagen. Das Konzept der Homotopie ist eine sehr allgemeine Relation in topologischen Räumen [6], [21], [140] und besagt, dass zwei Pfade $p$ und $q$ homotop zueinander sind, wenn sie kontinuierlich ineinander überführt werden können. Es muss also möglich sein, die eine in die andere Route zu verwandeln, ohne Hindernisse zu überspringen oder die Route aufzuteilen. In Abbildung 3.2 ist dies beispielsweise bei den Routen $q$ und $r$ der Fall. Zudem erzeugt die Homotopie eine Äquivalenzrelation, das bedeutet, sie erstellt eine disjunkte Partition der betrachteten Menge, in diesem Falle von Routen mit gleichem Start und Ziel, sodass anschließend jeder Pfad genau einer Äquivalenzklasse angehört. Diese Eigenschaft ist wünschenswert, da eine exakte Unterteilung in äquivalente und alternative Routen das Ziel ist. In Abbildung 3.2 wäre dies beispielsweise die „rote Klasse“, in der sich Route $p$ befindet, die „grüne Klasse“ ( $q$ und $r$ ), sowie die „blaue Klasse“ $(s)$. Da die Homotopie eine Äquivalenzrelation ist, gelten ebenso die drei Eigenschaften Reflexivität, Symmetrie und Transitivität. Damit ergibt sich jetzt folgende Definition von alternativen Routen: Wenn zwei Routen $p$ und $q$ homotop zueinander sind $(p \simeq q)$, so werden sie als äquivalent angesehen (siehe Gleichung 3.1). Wenn zwei Routen $p$ und $p$ nicht-homotop sind $(p \not q q)$, so gelten sie als Alternativen (Gleichung 3.2).

$$
\begin{aligned}
& p \simeq q \Rightarrow \text { äquivalent } \\
& p \nsucceq q \Rightarrow \text { alternativ }
\end{aligned}
$$




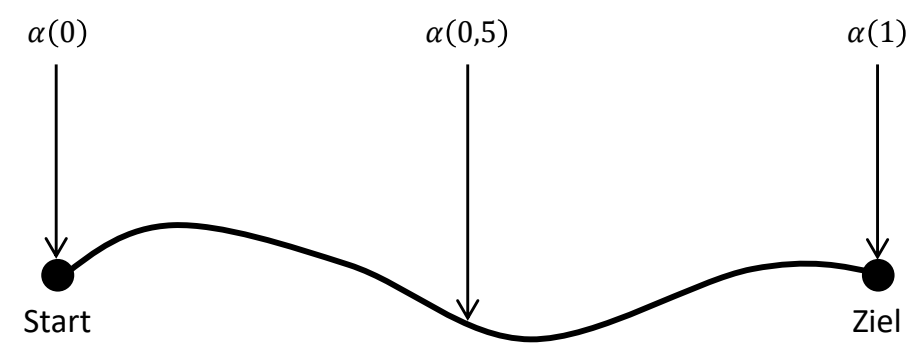

Abbildung 3.3: Pfad als kontinuierliche Abbildung im zweidimensionalen Raum.

Wie bereits erwähnt ist die Homotopie grundsätzlich eine sehr allgemeine Relation in topologischen Räumen. Im Kontext der komplexen Umgebungen beschränkt sich die Idee auf Pfade, die als kontinuierliche Abbildung im zweidimensionalen euklidischen Raum dargestellt werden können:

$$
\alpha:[0,1] \rightarrow \mathbb{R}^{2}
$$

Ein entsprechend Gleichung 3.3 dargestellter Pfad wird wie folgt interpretiert: Das Funktionsargument $t \in[0,1]$ repräsentiert die Zeit. Bei $t=0$ würde sich ein entlang des Pfades verlaufendes Objekt am Start befinden und bei $t=1$ am Ziel. Der Funktionswert $\alpha(t)$ repräsentiert den Ort, an dem sich das Objekt zur Zeit $t$ befindet. Somit wird mit $\alpha(0)$ der Startpunkt des Pfades $\alpha$ beschrieben und mit $\alpha(1)$ der Zielpunkt. Abbildung 3.3 verdeutlicht dies.

Zwei der obigen Definition entsprechende Pfade $\alpha$ und $\beta$ mit denselben Startund Zielpunkten sind nun homotop zueinander, wenn folgende kontinuierliche Abbildung

$$
\gamma:[0,1] \times[0,1] \rightarrow \mathbb{R}^{2}
$$

existiert und stets

$$
\begin{aligned}
& \gamma(t, 0)=\alpha(t), \\
& \gamma(t, 1)=\beta(t), \\
& \gamma(0, t)=\alpha(0)=\beta(0), \text { und } \\
& \gamma(1, t)=\alpha(1)=\beta(1)
\end{aligned}
$$

gilt. Eine solche Abbildung $\gamma$ wird Homotopie von $\alpha$ nach $\beta$ genannt. In dieser Abbildung beschreibt das erste Argument die Zeit in beiden Pfaden und das zweite Argument die Zeit im Verformungsprozess des ersten Pfades in den zweiten (siehe Abbildung 3.4). Die erste Bedingung, dargestellt in Gleichung 3.5, besagt nun, dass zu Beginn des Verformungsprozesses $\gamma$ exakt dieselben Punkte wie $\alpha$ zurückliefert, da $\alpha$ in $\beta$ überführt wird. Die zweite Bedingung 


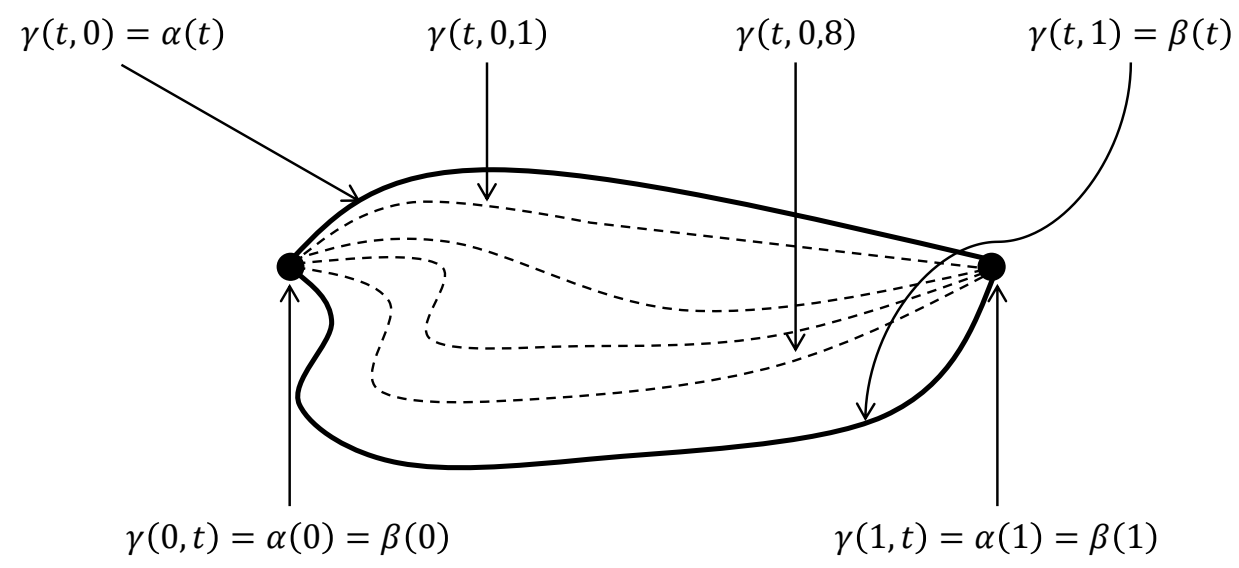

Abbildung 3.4: Beispiel einer Abbildung $\gamma$ als Homotopie von $\alpha$ nach $\beta$. Der obere Pfad $\alpha$ wird kontinuierlich (gestrichelte Linien) in den unteren Pfad $\beta$ deformiert.

3.6 besagt entsprechend, dass am Ende des Verformungsprozesses $\gamma$ exakt dieselben Punkte wie $\beta$ zurückliefert. Dies ist der Fall, wenn die kontinuierliche Überführung von $\alpha$ zu $\beta$ möglich war. Die dritte Bedingung 3.7 besagt schließlich, dass die Pfade einen identischen Startpunkt im zweidimensionalen Raum besitzen müssen. Analog besagt die vierte Bedingung 3.8, dass die Endpunkte der Pfade identisch sein müssen.

Wie bereits erläutert stellt die Homotopie eine Äquivalenzrelation auf der Menge aller betrachteten Pfade dar, sodass dies in einer disjunkten Aufteilung der Menge in Äquivalenzklassen resultiert. Jeder betrachtete Pfad gehört somit genau einer Äquivalenzklasse an, womit eine eindeutige Unterscheidung zwischen äquivalent und alternativ getroffen werden kann.

\subsubsection{Approximation der Homotopie}

Nachdem im vorigen Abschnitt eine Definition für alternative Routen in komplexen Umgebungen vorgestellt wurde, die das topologische Konzept der Homotopie verwendet, wird in diesem Abschnitt eine effiziente Methode zur Berechnung dieser Relation beschrieben.

Die Berechnung der Homotopie kann in mehrdimensionalen topologischen Räumen komplex sein, im zweidimensionalen euklidischen Raum kann mit entsprechenden Annahmen jedoch eine geeignete Annäherung berechnet werden. Grundsätzlich versucht der im weiteren Verlauf näher vorgestellte und bereits in [179] veröffentlichte Ansatz die Homotopie zwischen zwei Pfaden anzunähern, indem eine zusammenhängende Fläche erstellt wird. Dazu werden die zu untersuchenden Pfade verbunden und das Innere der Fläche untersucht. Wenn keine Hindernisse enthalten sind, so können die Begrenzungslinien der Fläche 
ineinander verformt werden, was bedeutet, dass die zwei Pfade homotop zueinander sind. Innerhalb dieser Arbeit werden für die effiziente Annäherung der Relation zwei Annahmen getroffen, die jedoch keine Exklusivität auf nur solche Konstellationen darstellen. Zum einen werden die zu untersuchenden Pfade nicht als parametrisierte Kurven, sondern als Streckenzug angesehen, also als eine Vereinigung von Strecken zwischen einer Folge von Punkten. Beliebige Kurven müssen demnach durch entsprechend viele Punkte dargestellt werden. Zum anderen wird als Kartendarstellung für beispielsweise einen Gebäudeplan ein Belegungsraster vorausgesetzt. Frei begehbare Fläche ist dabei weiß dargestellt, Hindernisse entsprechend in schwarz.

Der Ansatz wird an einem einfachen Beispiel in Abbildung 3.5 erläutert. Zu sehen sind zwei offensichtlich nicht-homotope Pfade und zwei Hindernisse (Abbildung 3.5a). Um zwei polygonale Linien ineinander überführen zu können, wird im ersten Schritt der eine Pfad mit dem anderen Pfad in umgekehrter Orientierung konkateniert. Diese Punktfolge beschreibt jetzt ein Polygon und wird mittels eines Polygon-Füll-Algorithmus ausgefüllt [109]. Die resultierende Maske wird in eine Matrix geschrieben, wobei eine 1 für das Innere des Polygons und eine 0 für die Fläche außerhalb des Polygons verwendet wird (Abbildung $3.5 \mathrm{~b}$ ). Als letzte vorbereitende Maßnahme muss die Karte mit den Hindernissen invertiert werden, sodass 0 eine frei begehbare Fläche repräsentiert und eine 1 entsprechend Hindernisse (Abbildung 3.5c). Nun folgt der eigentliche Test der Homotopie: die Polygonmaske wird elementweise mit der invertierten Karte multipliziert. Dieses elementweise Produkt (auch Hadamard-Produkt oder Schur-Produkt genannt) resultiert in einer Matrix, die ausschließlich aus Nullen besteht, wenn sich die Polygonmaske und die Hindernisgeometrie nicht überlagern. Falls sich jedoch Polygonmaske und Hindernisgeometrie überlagern (Abbildung 3.5d), so beinhaltet die Matrix an den entsprechenden Überlagerungsstellen Einsen. Insgesamt gilt also, dass, wenn die Matrix nur aus Nullen besteht, kein Hindernis zwischen den Routen vorliegt. Diese können damit kontinuierlich ineinander überführt werden, sind somit homotop zueinander und entsprechend keine alternative Routen. Wenn es hingegen von Null verschiedene Einträge in der Ergebnismatrix gibt, so ist eine Schnittmenge zwischen der Polygonmaske und der Hindernisgeometrie gegeben, womit die Routen nicht homotop zueinander sind. Sie können als Alternativen angesehen werden.

Folgende Gleichung fasst das Verfahren zusammen, um zu überprüfen, ob zwei Pfade $p$ und $q$ mit gleichem Start- und Zielpunkt homotop zueinander sind:

$$
p \simeq q \Leftrightarrow \operatorname{Maske}\left(p * q^{-1}\right) \circ(1-\text { Karte })=0
$$

Maske stellt eine entsprechende Funktion zum Füllen eines Polygons dar, * ist die Konkatenation von kompatiblen Pfadsegmenten, $q^{-1}$ ist der inverse Pfad von $q$ verlaufend vom Endpunkt zum Start, o ist das Hadamard-Produkt und der Term (1 - Karte) stellt die Invertierung der Karte dar.

Die obige Erläuterung ist für Polygone ohne Selbstschnitt eindeutig. Aber 


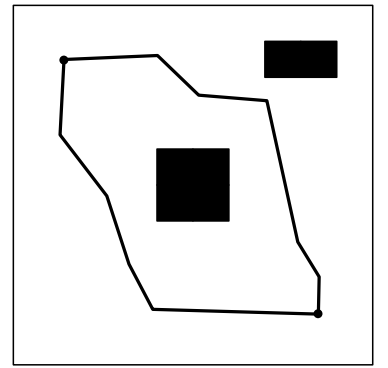

(a) Szenario mit zwei Routen und zwei Hindernissen.

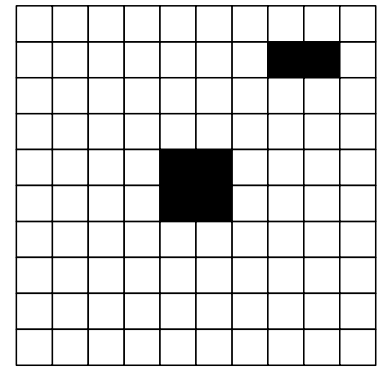

(c) Rasterdarstellung Hindernisse.

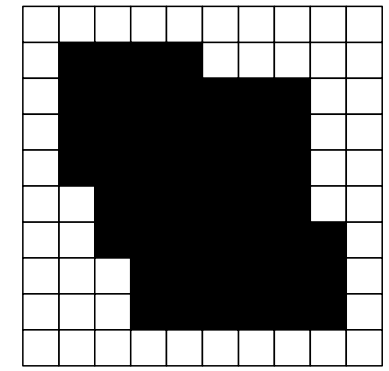

(b) Polygonmaske.

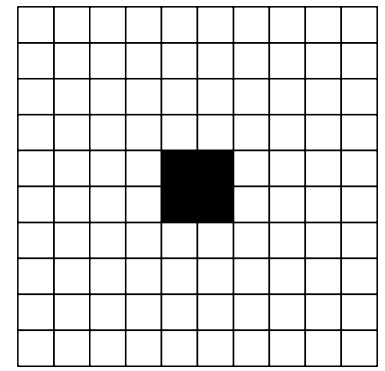

der(d) Schnittmenge von Polygonmaske und Hindernissen.

Abbildung 3.5: Darstellung der Approximation der Homotopie mittels Polygonmaske. 
auch für Polygone mit Selbstschnitt berechnen Polygon-Füll-Algorithmen die „richtigen“ Teilpolygone, wodurch ein „Innen“ und „Außen“ erhalten bleibt. Abschließend sei angemerkt, dass es geringfügig effizienter wäre, den eigentlichen Test auf Homotopie in den Polygon-Füll-Algorithmus zu integrieren. So könnte das Füllen des Polygons unterbrochen werden, sobald ein belegtes Pixel innerhalb des Polygons auftritt. In dem hier vorgestellten Weg wird dies jedoch nicht getan, da die Ergebnismatrix der elementweisen Multiplikation einen Hinweis auf die Anzahl der belegten Pixel innerhalb des Polygons gibt, was potentiell ein Bestandteil für ein Maß zur Berechnung der Unähnlichkeit von Routen sein kann.

\subsection{Heuristiken zum Finden nicht-homotoper Pfade}

Nachdem in den vorigen Abschnitten alternative Routen mittels des Konzeptes der Homotopie definiert und ein entsprechender approximierender Test beschrieben wurde, werden nun Algorithmen präsentiert, um solche nichthomotopen Pfade in komplexen Umgebungen zu berechnen. Dies stellt die dritte Herausforderung dar, die im Abschnitt 3.1 beschrieben wurde. Die im weiteren Verlauf dieser Arbeit vorgestellten Konzepte verwenden diese Algorithmen, um eine Menge von (alternativen) Routen in einem Belegungsraster $\mathrm{zu}$ berechnen.

Beide Algorithmen, die in den folgenden Abschnitten beschrieben werden, sind Erweiterungen von Verfahren, die auch im Straßennetz zum Finden alternativer Routen verwendet werden: der One-Patching-Algorithmus (in der Literatur oft Via-Node-Algorithmus genannt [2], [10]) und der PenaltyAlgorithmus [10], [30], [161].

Grundsätzlich fokussieren sich beide Ansätze auf folgende Problemstellung: Gegeben sei eine komplexe Umgebung in der Darstellung eines Belegungsrasters $A \subset \mathbb{B}^{m \times n}$, in der begehbare Fläche weiß und Hindernisse - innerhalb von Gebäuden einschließlich Wände und Möbel, jedoch ohne Türen - schwarz gefärbt sind, und ein Paar $(s, t)$ von Punkten, die mit ihren $x$ - und $y$-Koordinaten die Start- und Zielpunkte darstellen. Das Ziel ist das Berechnen einer Menge von $n$ vorzugsweise kurzen Pfaden $P=\left\{p_{1}, \ldots, p_{n}\right\}$ vom Start $s$ zum Ziel $t$, die paarweise nicht-äquivalent hinsichtlich der mittels der Homotopie berechneten Äquivalenzklassen sind. Darüber hinaus sind aus der Anwendungssicht Routen ohne Selbstschnitt von Interesse, da ein Anwender nicht „im Kreis“ laufen soll.

Sowohl der One-Patching-Algorithmus als auch der Penalty-Algorithmus wurden im Zuge der diesem Abschnitt zugrunde liegenden Veröffentlichung [179] implementiert, um zur Herstellung von Datensätzen, zur weiteren Verarbeitung und zur Analyse derselben verwendet werden zu können. Es wurde GNU Octave - eine Software inklusive einer höheren Programmiersprache, die insbesondere für numerische Berechnungen eingesetzt wird - als schnel- 
le Prototyping- und Evaluationsumgebung verwendet. Die Eingabe für den Aufruf von sowohl dem One-Patching- als auch Penalty-Algorithmus ist eine monochrome Bitmap, die eine komplexe Umgebungen wie beispielsweise ein Gebäude enthält, bei dem die Farbe Weiß begehbare Flächen und die Farbe Schwarz Hindernisse wie etwa Wände repräsentiert. Zudem sind die Start- und Zielpunkte als Pixelkoordinaten gegeben.

Für die Durchführung der Graphoperationen wie etwa das Lösen des Kürzester-Pfad-Problems wurde eine native Erweiterung für Octave entwickelt, die auf der Boost Graph Library (BGL) aufbaut [164]. BGL ist eine freie $\mathrm{C}++$-Bibliothek, die generische Graphoperationen einschließlich effizienter Implementierungen des Dijkstra-Algorithmus oder des $A^{*}$-Algorithmus beinhaltet.

Basierend auf der komplexen Umgebung, dargestellt als Belegungsraster, wird ein Graph wie folgt erstellt: Für jeden weißen Pixel wird ein Knoten erstellt. Für jedes Paar direkt benachbarter weißer Pixel wird eine Kante erstellt. Es werden sowohl die horizontalen und vertikalen Nachbarn berücksichtigt als auch die vier diagonalen Nachbarn. Die Kantengewichte für horizontale und vertikale Nachbarn wurden auf 1 gesetzt und folglich $\sqrt{2}$ für die Diagonalen.

\subsubsection{One-Patching-Algorithmus}

Gegeben ein Startpunkt $s$ und ein Zielpunkt $t$, so ist die Idee beim OnePatching-Algorithmus die Auswahl eines Stützpunktes $m$ innerhalb der navigierbaren Fläche (die Strategie der Auswahl wird weiter unten erläutert). Nun werden ein kürzester Pfad von $s$ nach $m$ und ein kürzester Pfad von $m$ nach $t$ berechnet, die anschließend konkateniert werden:

$$
p_{m}=\operatorname{ShortestPath}(s, m) * \operatorname{ShortestPath}(m, t)
$$

Der entstehende Pfad $p_{m}$ ist kein kürzester Pfad von $s$ nach $t$, es sei denn, der Stützpunkt $m$ liegt zufälligerweise auf dem kürzesten Pfad von $s$ nach $t$. Sukzessive werden nun weitere Routen bestehend aus zwei kürzesten Pfadsegmenten unter der Verwendung zusätzlicher Stützpunkte erstellt. Abbildung 3.6a deutet dies in einem kleinen Beispiel an. Zu sehen sind zwei Hindernisse (schwarze Rechtecke), Start- und Zielpunkte als blaue Diamanten, mehrere Stützpunkte als blaue Kreise sowie die entsprechend zusammengesetzten Routen. Für jede neue kombinierte Route von $s$ nach $t$ wird die Relation der Homotopie gegen alle zuvor erstellten Pfade geprüft. Wenn der Pfad homotop $\mathrm{zu}$ einem vorher gefundenen Pfad ist, so wird dieser verworfen (in Abbildung 3.6a werden homotope Pfade zur Veranschaulichung trotzdem angezeigt, jeweils in gleicher Farbe). Es sei angemerkt, dass das verwandte Problem der Suche des kürzesten homotopen Pfades in Bezug auf eine Referenzroute bereits in der Literatur gelöst wurde: es ist möglich, den kürzesten homotopen Pfad bezüglich einer Referenzroute in angemessener Zeit zu finden, siehe [18], [61], [105]. Aus diesem Grund ist die Qualität bezüglich der Länge der Pfade, 


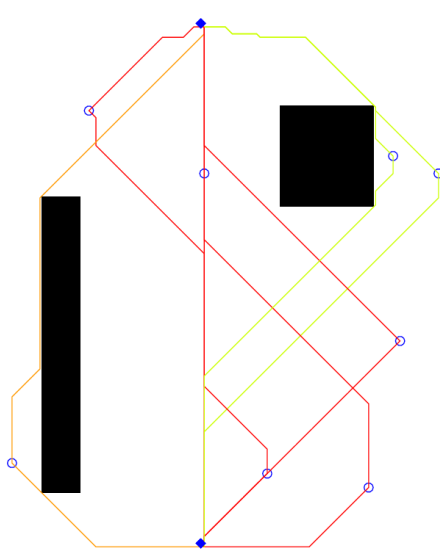

(a) Berechnete Routen.

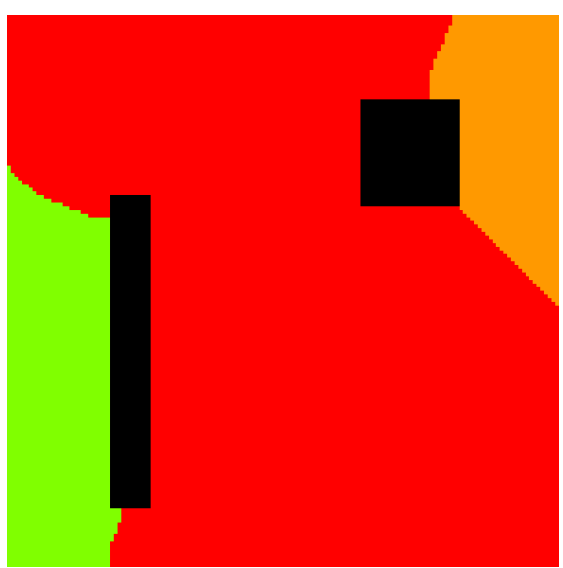

(b) Berechnete Referenzkarte.

Abbildung 3.6: Der One-Patching-Algorithmus verwendet Stützpunkte um Pfade zu erstellen, die aus der Konkatenation zwei kürzester Pfade bestehen.

die durch diesen Algorithmus berechnet werden, kein kritischer Faktor. Die berechneten Kandidaten können gespeichert und anschließend in einem Nachbearbeitungsschritt nachgebessert werden, indem beispielsweise der kürzeste Pfad jeder gefundenen Homotopieklasse berechnet wird.

Es wurden zwei Strategien implementiert, um die Sequenz der Stützpunkte zu definieren: Die erste Strategie ist der rasterbasierte Ansatz, bei dem jeder frei begehbare Punkt eines Rasters, das über der Kartendarstellung liegt, als Stützpunkt verwendet wird. Je nach Anzahl der durch das Raster ausgelassenen Pixel kann dies auf viele Berechnungsschritte hinauslaufen. Dieser Ansatz fokussiert sich also eher auf Vollständigkeit und ist für eine Offline-Analyse geeignet. Die zweite Strategie verwendet zufällig gewählte Stützpunkte (wie auch in Abbildung 3.6a). Dies führt im Mittel zu einer schnelleren Identifizierung neuer Homotopieklassen, besitzt jedoch keine Garantie über die Güte der Routen. Wenn die Stützpunkte zufällig gewählt werden, so liegen diese wahrscheinlich weiter voneinander entfernt als im rasterbasierten Ansatz, was wiederum die Wahrscheinlichkeit erhöht, dass die Polygonmaske der Routen nicht leer ist und deshalb nicht-homotope, alternative Routen gefunden werden. Diese Intuition wird in Abschnitt 3.3.3 evaluiert.

Eine Art Nebenprodukt des One-Patching-Algorithmus ist die Referenzkarte. Die Idee dabei ist, die Pixel in der Darstellung eines Belegungsrasters der gegebenen Karte in der Farbe einzufärben, die auch die Route haben würde, wenn das entsprechende Pixel als Stützpunkt genommen würde. In Abbildung 3.6b wurde dies für jedes Pixel der Karte getan. Mithilfe einer solchen Darstellung können Bereiche der Karte intuitiv erfasst werden. Offensichtlich gibt es einen großen Bereich, der mittig durch beide Hindernisse führt, aber auch zwei kleinere Bereiche, die zu Routen anderer Homotopieklassen führen. Im Grunde 


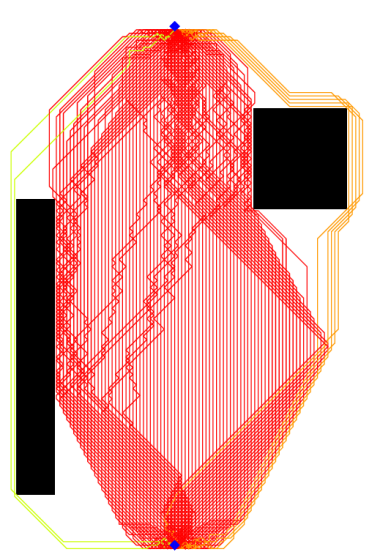

(a) Berechnete Routen.

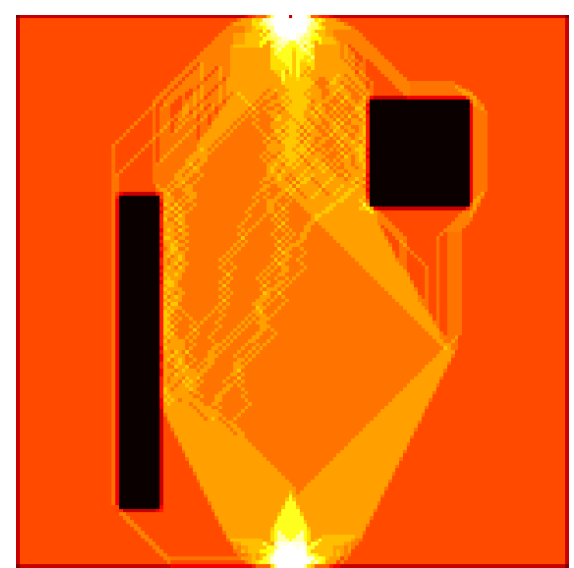

(b) Berechnete Gewichtskarte.

Abbildung 3.7: Der Penalty-Algorithmus erhöht die Kantengewichte von kürzesten Pfaden, um so an möglichst verschiedene kurze Routen zu gelangen.

sieht man hier: um von oben nach unten zu gelangen, kann man entweder links an den Hindernissen vorbei, mittig hindurch oder rechts vorbeigehen.

\subsubsection{Penalty-Algorithmus}

Der zweite in [179] vorgestellte sowie in der vorliegenden Arbeit verwendete Ansatz zum Finden von alternativen Routen basiert auf den Ansätzen des Penalty-Algorithmus, der auch im Umfeld der Routenfindung in Straßennetzen verwendet wird. Der Algorithmus soll Routen finden, die vom kürzesten Pfad beziehungsweise vom Referenzpfad verschieden, aber dennoch kurz sind. Die Grundidee dabei ist zwischen den folgenden zwei Schritten zu iterieren: Berechnung des kürzesten Pfades und Erhöhung der Kantengewichte der im kürzesten Pfad enthaltenen Kanten. Dies bewirkt, dass der vormals kürzeste Pfad stetig länger wird, bis der ursprünglich zweitkürzeste Pfad nun der Kürzeste ist. Abbildung 3.7a verdeutlicht dies. In der ersten Iteration wird der kürzeste Pfad zwischen dem Start und Ziel (blaue Diamanten) berechnet. Dieser verläuft entsprechend der direkten Luftlinie zwischen den blauen Diamanten von oben nach unten. Nach der Erhöhung der Kantengewichte aller vom ersten Pfad verwendeten Kanten wird erneut der kürzeste Pfad vom Start zum Ziel berechnet. Dies ist nun nicht mehr der zuletzt gefundene Pfad, sondern eine Abweichung davon. In den ersten Iterationen werden also die unterschiedlichen Variationen mittig zwischen den zwei Hindernissen gefunden. In den weiteren Iterationen wird dieser Bereich immer „unattraktiver" beziehungsweise „teurer“, sodass schließlich der kürzeste Pfad vom Start zum Ziel beispielsweise rechts an den Hindernissen vorbei läuft (orange Routen).

Die wichtigste Konfigurationsmöglichkeit beim Einsatz des PenaltyAlgorithmus ist die Art und Weise der Erhöhung der Kantengewichte. Wenn die 
Erhöhung der Kantengewichte zu gering ist, so werden mehr Iterationsschritte benötigt, bis eine Route innerhalb einer neuen Homotopieklasse gefunden wird. Falls die Erhöhung zu stark angesetzt ist, so können Teile des Graphen derart bestraft werden, dass „nützliche“ Stellen in der komplexen Umgebung (beispielsweise eine Brücke) von Routen nicht mehr durchlaufen werden. Somit können große Mengen von Routen wegfallen. Im weiteren Verlauf der Arbeit wurden Routen weiterverarbeitet, die mit der Strategie der Verdoppelung der Kantengewichte erstellt wurden.

Wie auch der One-Patching-Algorithmus erzeugt der Penalty-Algorithmus eine Art Nebenprodukt, nämlich die Gewichtskarte. Hierbei werden die Kantengewichte derart in die Karte gezeichnet, dass im Stile einer Heatmap hohe Gewichte eine helle („heiße“) Farbe erhalten und Kanten mit niedrigeren Gewichten entsprechend dunklere (,kalte“) Farben. In Abbildung 3.7b ist dies dargestellt.

\subsubsection{Evaluation der Heuristiken zum Finden nicht-homotoper Pfade}

In diesem Abschnitt sollen der One-Patching-Algorithmus und der PenaltyAlgorithmus hinsichtlich des relativen Laufzeitverhaltens beim Finden von Routen unterschiedlicher Homotopieklassen evaluiert und die einzelnen Vorund Nachteile diskutiert werden. Hierzu werden drei Szenarien verwendet, um das Verhalten der Algorithmen zu erklären. Das erste Szenario „Simple“ soll ein grundlegendes Verständnis von der Funktionsweise der beiden Algorithmen vermitteln. Es besteht aus einer quadratischen, frei begehbaren Fläche, in der zwei einfache Hindernisse angeordnet sind. Der Startpunkt ist horizontal zentriert im oberen Bereich der Karte angeordnet, der Zielpunkt horizontal zentriert im unteren Bereich. Unter der vereinfachenden Annahme, dass auf der Anwendungsebene nur Routen ohne Selbstschnitt von Interesse sind (der Anwender soll nicht im Kreis laufen), sind in dem Szenario „Simple“ drei sinnvolle Homotopieklassen enthalten: links um die Hindernisse herum, mittig hindurch und rechts herum. Das zweite Szenario namens „Labyrinth“ wurde ausgewählt, um zu zeigen, dass der One-Patching-Algorithmus ausschließlich Routen findet, die aus einer Verbindung von zwei kürzesten Pfaden resultieren. Anders als der Penalty-Algorithmus findet dieser also nicht die Klasse an Routen, die durch beide Labyrinthe führt. Schließlich stellt das Szenario „White House“ ein realistischeres Szenario einer komplexen Umgebung dar, nämlich einen vereinfachten historischen Gebäudeplan eines Teils des Weißen Hauses.

\subsubsection{Szenario „Simple“}

Abbildung 3.8 zeigt die Evaluationsergebnisse für das Szenario „Simple“. Der zufallsbasierte One-Patching-Algorithmus wurde mit 100 Iterationen konfiguriert, was entsprechend 100 zufällig gewählten Stützpunkten entspricht. Um 
den Zufallsfaktor zu kompensieren, wurde ein solches Experiment 100 Mal durchgeführt. Abbildung 3.8a zeigt eines dieser Ergebnisse. Der rasterbasierte One-Patching-Algorithmus (nicht abgebildet) wurde mit drei unterschiedlichen Rastergrößen durchgeführt, was in 100, 49 sowie 36 Iterationsschritten resultiert. Schließlich wurde auch der Penalty-Algorithmus mit einer fest eingestellten Anzahl von 100 Iterationsschritten ausgeführt (siehe Abbildung 3.8b).

Die Effizienz der drei Algorithmen für das Szenario „Simple“ ist in Abbildung 3.8c dargestellt. Auf der x-Achse ist die Anzahl der Iterationsschritte abgetragen und auf der y-Achse die Anzahl der zu diesem Zeitpunkt gefundenen unterschiedlichen Homotopieklassen. Der Penalty-Algorithmus (blaue gestrichelte Linie) findet die drei zu erwartenden Homotopieklassen (links, mitte, rechts) innerhalb des definierten Versuchsaufbaus. Die erste Klasse wird selbstverständlich im ersten Iterationsschritt gefunden. Es ist der kürzeste Pfad zwischen dem Start- und Zielpunkt, also die Route, die mittig durch beide Hindernisse verläuft. Die zweite Homotopieklasse wird nach 77 Iterationsschritten gefunden, direkt gefolgt von der dritten Klasse nach 79 Schritten. Die relativ lange Zeitspanne zwischen der ersten und zweiten Klasse beruht auf der Tatsache, dass die Karte eine große Freifläche in der Mitte besitzt. Bevor nach der ersten Klasse eine weitere gefunden wird, muss somit erst der mittlere Bereich stark genug „bestraft“ werden. Die zweite und dritte Klasse werden schnell hintereinander gefunden, da die Längen der beiden Alternativen recht ähnlich sind. Dies deutet auf eine Schwäche des Penalty-Algorithmus hin: wenn die Länge der nächsten zu findenden alternativen Route stark von der Länge der aktuell kürzesten Route abweicht, so sind viele Iterationsschritte notwendig.

Um die Ergebnisse des Penalty-Algorithmus mit denen des rasterbasierten One-Patching-Algorithmus zu vergleichen, wurde eine Rastergröße gewählt, die in derselben Anzahl an Iterationsschritten wie bei dem Penalty-Ansatz resultiert (100 Iterationen). Da der rasterbasierte One-Patching-Algorithmus den Penalty-Algorithmus in vielen durchgeführten Experimenten hinsichtlich der Leistung übertrifft, wurden zusätzliche Durchläufe mit größerem Raster und entsprechend weniger Iterationsschritten durchgeführt (49 und 36 Iterationsschritte). In allen drei Konfigurationen findet der rasterbasierte One-PatchingAlgorithmus die zweite Homotopieklasse sehr schnell, nämlich nach 8, 6 und 5 Schritten (siehe grüne durchgezogene Linien in Abbildung 3.8c). Auch die dritte Homotopieklasse wird schnell gefunden, da die Hindernisse jeweils nach wenigen Zeilen des Rasters erscheinen.

Schließlich wurde das Verhalten des zufallsbasierten One-PatchingAlgorithmus untersucht. Wie bereits erwähnt wurden 100 Experimente mit je 100 Iterationsschritten ausgeführt. In jedem Durchlauf wurden die drei zu erwartenden Homotopieklassen gefunden (siehe rote Kreise in Abbildung 3.8c). Im Durchschnitt wurden 13,7 Iterationsschnitte benötigt, um drei Homotopieklassen zu finden (Median: 12, Minimum: 3, Maximum: 41). Im Gegensatz zum Penalty-Algorithmus wurde die zweite Klasse extrem schnell nach durchschnittlich 5,8 Iterationsschritten gefunden (Median: 4, Minimum: 2, Maxi- 
mum: 25). Es sei angemerkt, dass die Implementierung des zufallsbasierten One-Patching-Algorithmus nicht immer mit dem ersten Iterationsschritt eine Homotopieklasse findet, da der zufällig gewählte Stützpunkt ein schwarzes Pixel - also nicht begehbar - sein kann oder der Stützpunkt nicht zwingend vom Start oder dem Ziel erreichbar sein muss. Diese Möglichkeiten resultieren darin, dass der jeweilige Iterationsschritt keine Route findet.

Zusammengefasst kann gesagt werden, dass der zufallsbasierte OnePatching-Algorithmus eine sehr gute Strategie für einfache Szenarien ist. Aber auch die anderen Algorithmen waren in der Lage, in einer angemessenen Zeit alle drei zu erwartenden Homotopieklassen (links, mitte, rechts) im Szenario „Simple“ zu finden.

Nachteilig aufgefallen sind beim Penalty-Algorithmus der teils hohe Berechnungsaufwand sowie gewisse Artefakte am Anfang und am Ende der Routen. Dort wurden nämlich die Knoten derart oft bestraft, was zu verworrenen Bewegungen der Pfade führte. Ein ähnliches Artefakt können die Routen des One-Patching-Algorithmus in der Nähe der Stützpunkte zeigen, da dort eine Konkatenation der zwei kürzesten Pfade stattfindet, was möglicherweise einen Umweg darstellt.

\subsubsection{Szenario „Labyrinth“}

Das Szenario „Labyrinth“ (siehe Abbildung 3.9) wurde innerhalb der Vorveröffentlichung [179] als Beispiel konzipiert, bei dem der Penalty-Algorithmus dem One-Patching-Algorithmus überlegen ist, da ersterer mehr Homotopieklassen findet. Das Szenario besteht aus zwei übereinander angeordneten Labyrinthen, wobei sich der Startpunkt mittig an der oberen Seite der Karte befindet und der Zielpunkt mittig an der unteren Seite, und enthält mindestens die folgenden neun Homotopieklassen: beide Labyrinthe werden entweder komplett linksseitig $(C 1)$ oder komplett rechtsseitig $(C 2)$ umlaufen; das obere Labyrinth wird durchlaufen und das untere Labyrinth wird entweder linksseitig $(C 3)$ oder rechtsseitig umlaufen $(C 4)$; das obere Labyrinth wird entweder linksseitig $(C 5)$ oder rechtsseitig $(C 6)$ umlaufen, während das untere Labyrinth in jedem Fall durchlaufen wird; beide Labyrinthe werden durchlaufen $(C 7)$; das obere Labyrinth wird linkseitig umlaufen und das untere Labyrinth rechtsseitig $(C 8)$; das obere Labyrinth wird rechtseitig umlaufen und das untere Labyrinth linksseitig $(C 9)$.

Der Penalty-Algorithmus findet fünf der soeben beschriebenen Homotopieklassen innerhalb der festgelegten Iterationsanzahl von 250 (siehe Abbildung 3.9a für mehrere Momentaufnahmen der Ausführung): Die als erstes gefundene Klasse $C 1$ wird nach einem Iterationsschritt gefunden, gefolgt von Klasse $C 2$ nach sechs Schritten. Die darauf folgenden Klassen $C 3$ und $C 4$ werden nach 79 beziehungsweise 104 Schritten gefunden. Es werden also die Routen, die beide Labyrinthe umlaufen, gefunden sowie die Routen, die durch das obere Labyrinth durchlaufen und das untere Labyrinth umlaufen. Der umgekehrte Fall, nämlich das Umlaufen des oberen und das Durchlaufen des unteren Labyrinths 


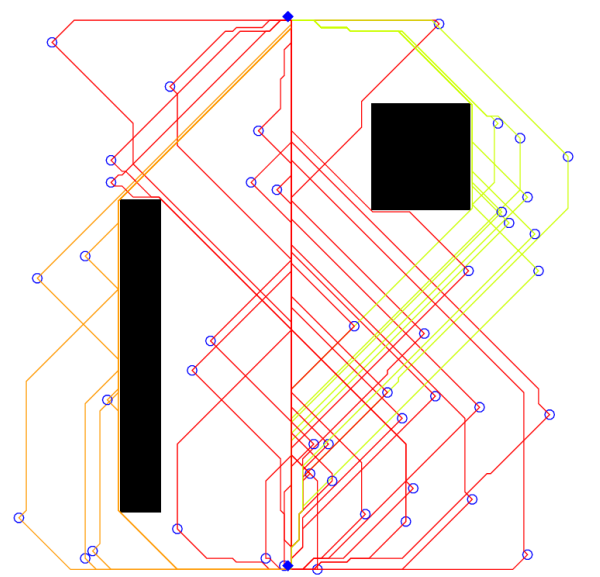

(a) Beispielhaftes Ergebnis für den OnePatching-Algorithmus mit 100 zufällig gewählten Stützpunkten.

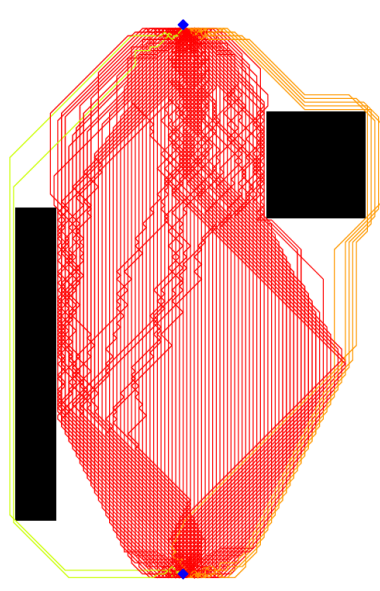

(b) Ergebnis für den Penalty-Algorithmus mit 100 Iterationsschritten.

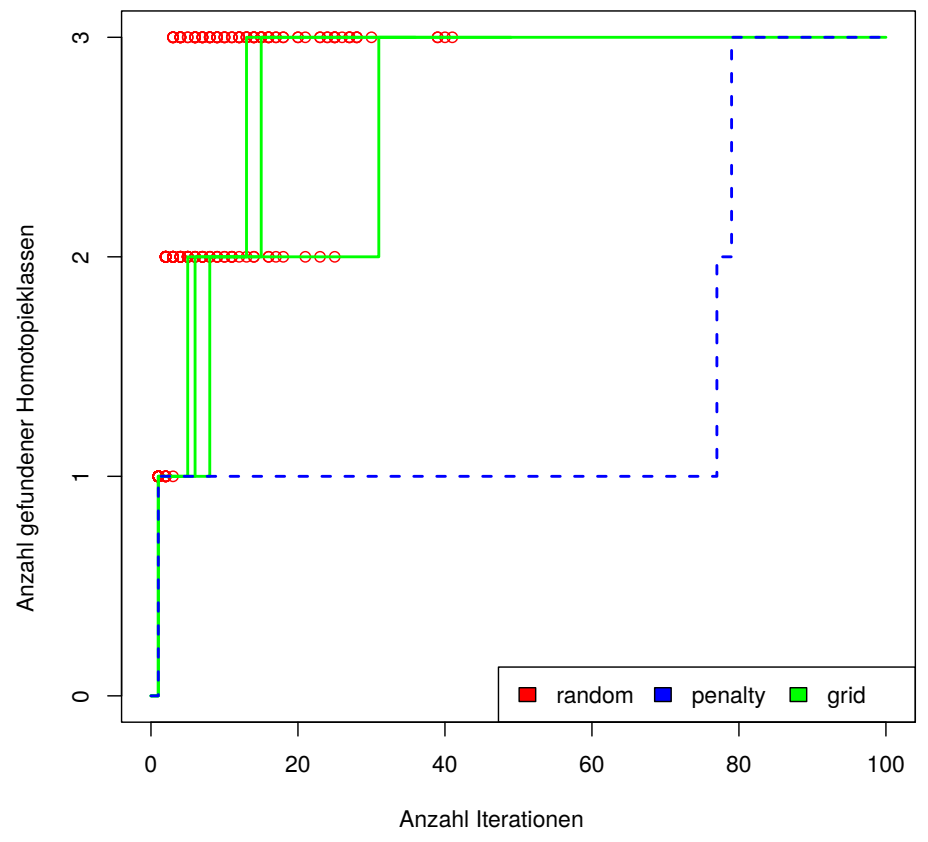

(c) Effizienz der unterschiedlichen Algorithmen. Die $x$-Achse repräsentiert den Iterationsschritt und die $y$-Achse die Anzahl der bis dahin gefundenen Homotopieklassen.

Abbildung 3.8: Evaluationsergebnisse für das Szenario „Simple“. 
(C5 und C6), tritt nicht auf. Nach 219 Iterationsschritten findet der PenaltyAlgorithmus schließlich die Klasse $C 7$, bei der die Routen beide Labyrinthe durchlaufen. Dies ist wie bereits beschrieben ein Unterscheidungsmerkmal zum One-Patching-Algorithmus, da dieser eine solche Homotopieklasse nicht finden kann: eine solche Route ist keine Konkatenation von zwei kürzesten Pfaden. Der Penalty-Algorithmus findet jedoch nicht die S-förmigen Klassen $C 8$ und $C 9$.

Das Ergebnis des rasterbasierten One-Patching-Algorithmus ist in Abbildung $3.9 \mathrm{~b}$ dargestellt. Zur besseren Lesbarkeit wurden die gefundenen Routen gesondert nach deren Homotopieklasse visualisiert. Es ist zu erkennen, dass der One-Patching-Algorithmus nur solche Routen findet, die aus der Konkatenation von zwei kürzesten Pfaden bestehen, das heißt entweder beide Labyrinthe umlaufen ( $C 1$ und $C 2$ ) oder nur eines der Labyrinthe durchlaufen ( $C 4$ und $C 5$ ). Die zu $C 4$ und $C 5$ symmetrischen Klassen $C 3$ und $C 6$ werden aufgrund der Anordnung der zwei Labyrinthe nicht gefunden.

In Abbildung 3.9c sind die zahlenmäßigen Ergebnisse des Versuchsaufbaus „Labyrinth“ zu sehen, die sich ähnlich zu denen vom Szenario „Simple“ verhalten. Der rasterbasierte One-Patching-Algorithmus (grüne durchgezogene Linien) findet zwar mit geringfügig weniger Iterationsschritten als der PenaltyAlgorithmus (blaue gestrichelte Linie) neue Homotopieklassen, jedoch nur vier verschiedene Klassen $(C 1, C 2, C 4, C 5)$. Der zufallsbasierte One-PatchingAlgorithmus (rote Kreise) findet erneut sehr schnell neue Homotopieklassen. In 72 der 100 durchgeführten Versuche werden vier Klassen gefunden $(C 1, C 2$, $C 4, C 5)$ und in den verbleibenden 28 Durchläufen fünf Klassen (ebenfalls $C 1$, $C 2, C 4, C 5$ und zusätzlich eine der S-förmigen Klassen $C 8$ oder $C 9$ ). Wie vermutet findet der zufallsbasierte One-Patching-Algorithmus schnell neue Klassen. Im Durchschnitt werden 104,8 Iterationsschritte benötigt, um fünf Klassen zu finden (Median: 79,5, Minimum: 12, Maximum: 222). Vier Klassen werden durchschnittlich in 31,2 Schritten gefunden (Median: 24, Minimum: 4, Maximum: 105), drei Klassen in durchschnittlich 11,2 Schritten (Median: 7) und zwei Klassen in durchschnittlich 2,8 Iterationsschritten (Median: 2).

\subsubsection{Szenario ,White House“}

Schließlich soll das Verhalten der Algorithmen mit einem realistischeren Szenario einer komplexen Umgebung untersucht werden, nämlich mit einem vereinfachten historischen Gebäudeplan eines Teils des Weißen Hauses. Die Ergebnisse sind in Abbildung 3.10 dargestellt. Der Startpunkt befindet sich im Raum in der oberen linken Ecke und das Ziel im Raum unten rechts. Innerhalb der vorgegebenen 250 Iterationsschritte findet der Penalty-Algorithmus insgesamt 18 unterschiedliche Homotopieklassen, davon 12 Klassen innerhalb der ersten 100 Iterationsschritte. Im Grunde sind dies die Routen, die durch die oberen Räume des Gebäudes führen sowie durch den langen, horizontalen Korridor. Es folgen etwa 70 Iterationsschritte, in denen keine neuen Homotopieklassen gefunden werden. Schließlich findet der Algorithmus die verbleibenden 

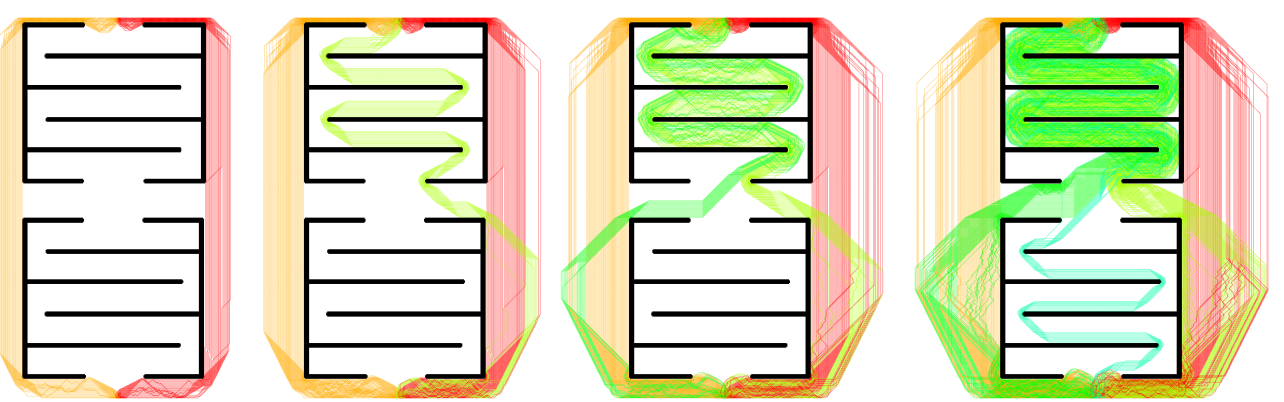

(a) Unterschiedliche Momentaufnahmen bei der Durchführung des Penalty-Algorithmus mit 250 Iterationsschritten.
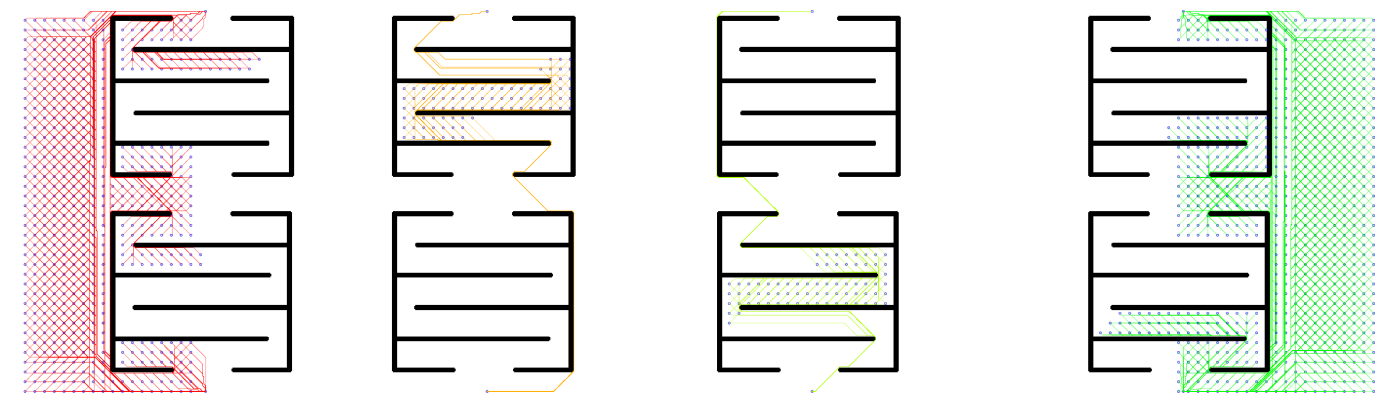

(b) Ergebnis des rasterbasierten One-Patching-Algorithmus. Zur Besserung Sichtbarkeit wurden die Routen der unterschiedlichen Homotopieklassen gesondert dargestellt.

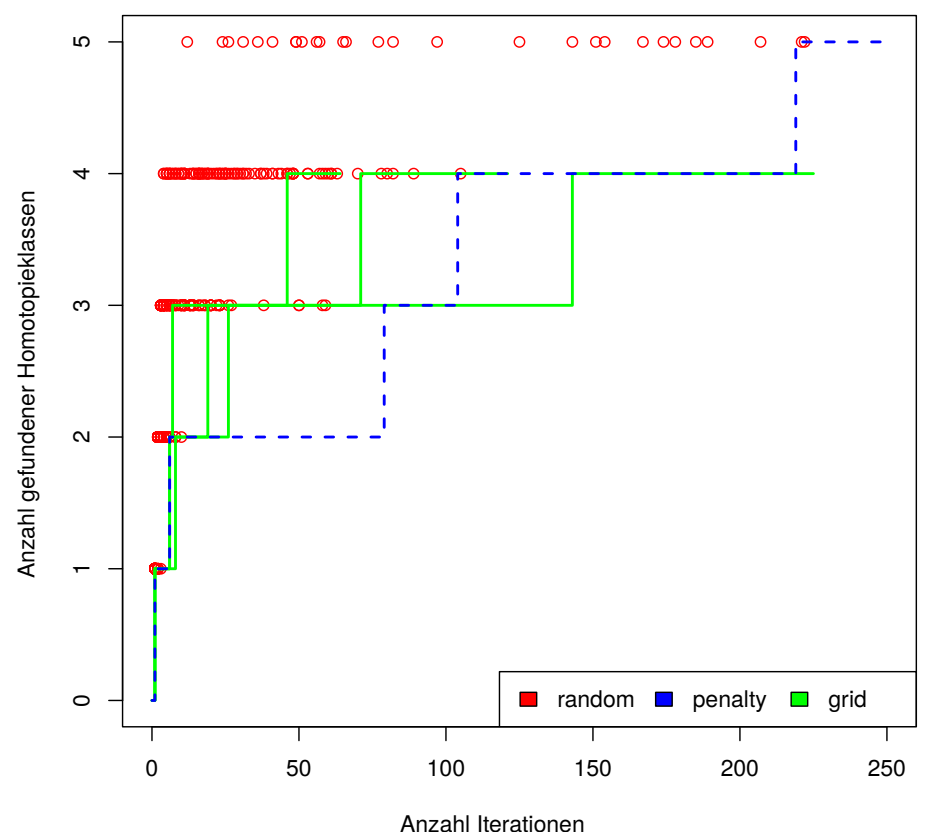

(c) Effizienz der unterschiedlichen Algorithmen. Die $x$-Achse repräsentiert den Iterationsschritt und die $y$-Achse die Anzahl der bis dahin gefundenen Homotopieklassen.

Abbildung 3.9: Evaluationsergebnisse für das Szenario „Labyrinth“. 
sechs Klassen an Routen. Diese führen durch den ovalen Raum und über den darunterliegenden Balkon. In Abbildung 3.10a sind die resultierenden Routen dargestellt, jedoch zur besseren Visualisierung je eine pro Homotopieklasse.

Wenn ein Raster gewählt wird, sodass 260 Iterationsschritte zur Verfügung stehen, findet der rasterbasierte One-Patching-Algorithmus 15 unterschiedliche Homotopieklassen. Selbst mit einem Raster mit größerer Kantenlänge und demnach weniger Iterationsschritten (beispielsweise 140 und 77 Iterationsschritte, siehe grüne Linien in Abbildung 3.10d) findet der Algorithmus nur 12 unterschiedliche Homotopieklassen. Der zufallsbasierte One-PatchingAlgorithmus wurde 100 mal mit jeweils 250 Iterationsschritten ausgeführt und war in der Lage, 12 Klassen mit durchschnittlich 116,6 Schritten zu finden (Median: 109, Minimum: 35, Maximum: 247).

Wie bereits in der Beschreibung der Algorithmen angedeutet, ist die Visualisierung der Kantengewichte ein Nebenprodukt des Penalty-Algorithmus. In Abbildung 3.10c ist dies für den Zustand des Graphen nach 250 Iterationsschritten zu sehen. Es ist leicht zu erkennen, dass die Routen im linken Teil des Gebäudes in mehr Variationen verlaufen als im rechten Bereich. Tatsächlich ist es so, dass es im rechten Bereich des Gebäudes eine Tür gibt, die definitiv passiert werden muss, wenn der Start- mit dem Zielpunkt verbunden werden soll. Diese Beobachtung motiviert den Abschnitt 4.3 zur Verwendung einer Überlastungswahrscheinlichkeit zur Bewertung von Routen.

\subsubsection{Zusammenfassende Bewertung}

Die dargestellten Heuristiken zum Finden nicht-homotoper Pfade haben Vorund Nachteile, die je nach Anwendungsfall stärker ins Gewicht fallen.

Beim Penalty-Algorithmus ist von Vorteil, dass die ermittelten Routen grob sortiert basierend auf deren Länge sind. In jedem Iterationsschritt wird der jeweils aktuelle kürzeste Pfad berechnet. Ein weiterer Vorteil gegenüber dem One-Patching-Algorithmus ist die Tatsache, dass dieser auch Homotopieklassen findet, die nicht als Verbindung von zwei kürzesten Pfaden ausgedrückt werden können. Im Szenario „Labyrinth“ ist der Penalty-Algorithmus entsprechend in der Lage, auch die Klasse an Routen $\mathrm{zu}$ finden, die durch beide Labyrinthe verläuft. Die Gewichtskarte, also die Visualisierung der während der Durchführung des Algorithmus erhöhten Kantengewichte, ist ebenfalls ein Vorteil, da dies einen einfachen visuellen Zugang zur Struktur der Karte ermöglicht. Diese Beobachtungen sind auch Motivation zur Verwendung einer Überlastungswahrscheinlichkeit zur Bewertung von Routen (siehe Abschnitt $4.3)$.

Ein großer Nachteil des Penalty-Algorithmus sind dessen hohen Berechnungskosten. In jedem Iterationsschritt erwirkt die Erhöhung der Kantengewichte einen neuen Graphen, sodass in jedem Iterationsschritt erneut der Kürzeste-Pfad-Baum berechnet wird. Eine angemessene Art und Weise der Erhöhung der Kantengewichte kann stark von der verwendeten Karte abhängen. 


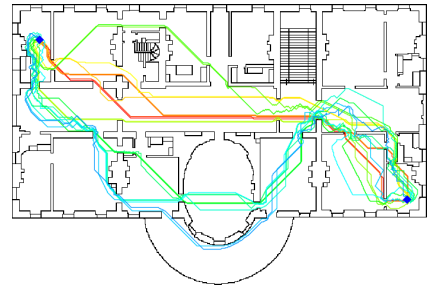

(a) Ergebnis für den PenaltyAlgorithmus mit 250 Iterationsschritten.

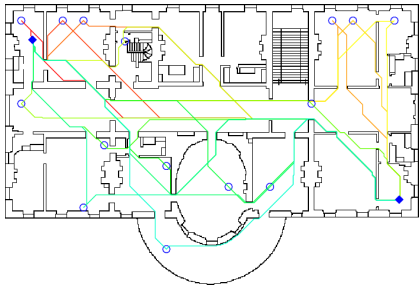

(b) Ergebnis für den OnePatching-Algorithmus mit einem Raster, das in 260 Iterationsschritte resultiert.

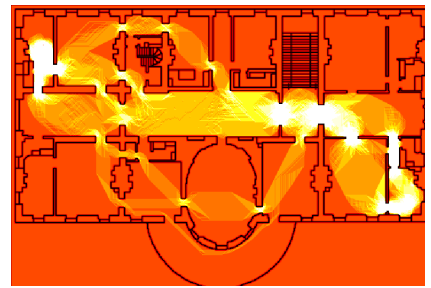

(c) Gewichtskarte, die die Kantengewichte nach Ausführung des Penalty-Algorithmus mit 250 Iterationsschritten visualisiert.

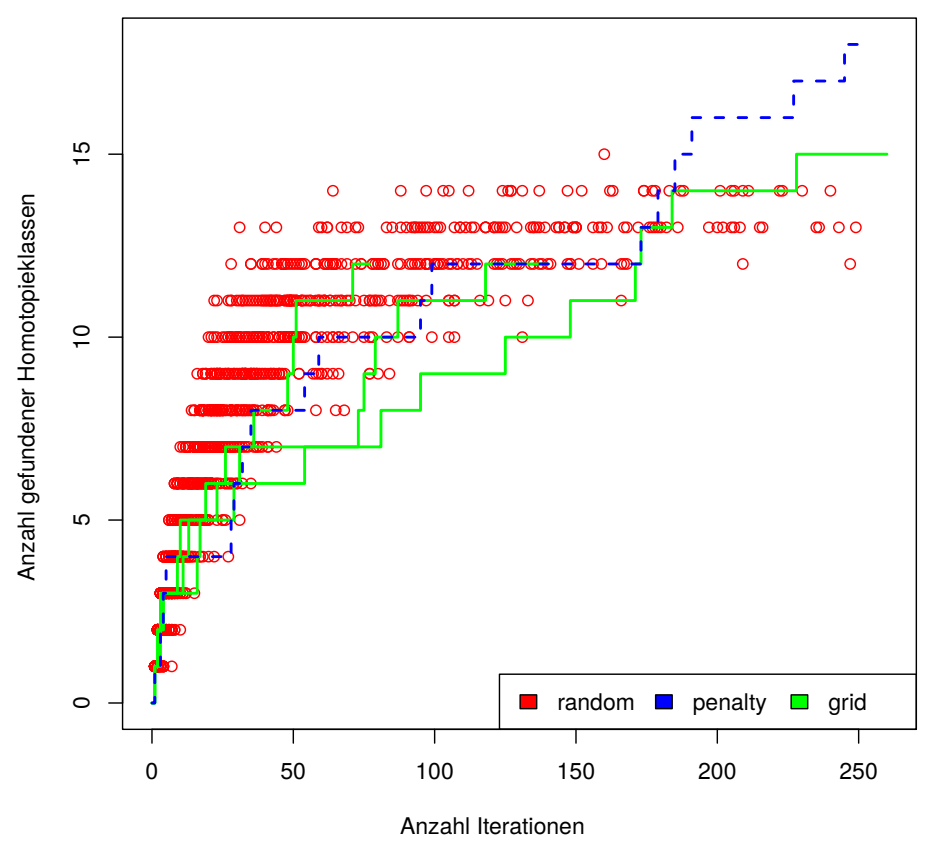

(d) Effizienz der unterschiedlichen Algorithmen. Die $x$-Achse repräsentiert den Iterationsschritt und die $y$-Achse die Anzahl der bis dahin gefundenen Homotopieklassen.

Abbildung 3.10: Evaluationsergebnisse für das Szenario „White House“. 
Wie bereits erwähnt, wurde für die Evaluation und für den weiteren Verlauf der Arbeit die Strategie der Verdoppelung der Kantengewichte gewählt. Eine weitere Schwäche des Penalty-Algorithmus zeigt sich, wenn die Länge der nächsten zu findenden alternativen Route stark von der Länge der aktuell kürzesten Route abweicht. In diesem Fall sind viele Iterationsschritte notwendig, bis die gefundene Route in einer neuen Homotopieklasse liegt.

Der enorme Vorteil des One-Patching-Algorithmus ist die Tatsache, dass für die komplette Analyse eines Belegungsrasters lediglich zwei vorberechnete Kürzester-Pfad-Bäume ausreichen. Da sich die Gewichte der Kanten nicht verändern, muss auch kein neuer Graph erstellt werden. Ein weiterer Vorteil sind die verschiedenen zur Verfügung stehenden Varianten des One-PatchingAlgorithmus, die je nach Anwendungsfall eingesetzt werden können. Mit der rasterbasierten Variante können alle Äquivalenzklassen der beschriebenen Art entdeckt werden, jedoch mit dem Preis eines erhöhten Mehraufwands an Berechnung. Der zufallsbasierte Ansatz kann in Szenarien verwendet werden, in denen schnell viele Klassen von alternativen Routen notwendig sind. Die Ergebnisse sind wesentlich schneller verwendbar. Schließlich ist die Referenzkarte eine Art positives Nebenprodukt. Diese färbt Punkte einer Karte gleich ein, wenn die Verwendung dieser Punkte als Stützpunkte zu Routen in gleichen Homotopieklassen führen. Diese Visualisierung gibt einen schnellen und intuitiven Einblick in die Struktur der Karte.

Der wohl größte Nachteil des One-Patching-Algorithmus ist die Tatsache, dass dieser nur Äquivalenzklassen finden kann, deren Routen auf der Konkatenation von zwei kürzesten Pfaden beruhen. Dies wurde im Szenario mit den Labyrinthen demonstriert.

Nachteilig bei beiden Verfahren ist die Notwendigkeit einer Nachbearbeitung der resultierenden Routen. Der Penalty-Algorithmus gibt Routen zurück, die Artefakte am Anfang und am Ende besitzen. Dort wurden die Kantengewichte derart oft erhöht, dass es zu verworrenen Bewegungen der Pfade führt. Im Gegensatz dazu können die Routen des One-Patching-Algorithmus ein unnatürliches Verhalten in der Nähe der Stützpunkte zeigen, da dort eine Konkatenation der zwei kürzesten Pfade stattfindet, was möglicherweise einen Umweg darstellt. Ein weiterer Nachteil bei beiden Heuristiken ist die Definition eines angemessenen Terminierungskriteriums, um die Berechnung der Algorithmen zu stoppen. Dies kann beispielsweise über die Begrenzung der Anzahl der Iterationsschritte erreicht werden, über die Anzahl der zu findenden Routen, über die Anzahl der zu findenden Homotopieklassen oder beispielsweise über die vom Algorithmus erkundete Fläche beziehungsweise Größe des Graphen. 


\subsection{Auswahlkriterien für Alternativen in Straßennetzen}

Wie bereits in Kapitel 2 erläutert, ist die Berechnung von kürzesten Pfaden innerhalb von Straßennetzen ein seit Jahren stark untersuchter Forschungsbereich. Dies führte zur Erforschung von Techniken zur Ausführungsbeschleunigung, sodass Echtzeitanwendungen für die kontinentweite Wegfindung ermöglicht wurden [11]. Darüber hinaus hat sich ein weiterer, jedoch weitaus weniger stark untersuchter Forschungsbereich entwickelt, nämlich die Berechnung von alternativen Routen. Hintergrund ist die Tatsache, dass oftmals mehrere, hochgradig unterschiedliche Wege zwischen zwei Punkten existieren, die zudem annähernd so gut wie der kürzeste Pfad sind.

Getreu dem Motto „Human-in-the-loop“ hat die Berechnung einer kleinen Menge von qualitativ hochwertigen alternativen Routen den Zweck, den Anwender nach eigenen Präferenzen wählen zu lassen (siehe auch Abschnitt 2.2.4). Die Aggregation von wenigen alternativen Routen in einer kompakten Darstellung wird Alternativgraph genannt. Für die Erstellung eines solchen Alternativgraphen muss entschieden werden, wann ein Routenkandidat in den Graphen integriert wird und wann nicht. Hierzu wird eine Qualitätsmetrik benötigt, mithilfe derer eine Zielfunktion optimiert wird. Damit wird dann die Erstellung von Alternativgraphen bewertet und die beste Instanz zurück geliefert.

Die Überprüfung der Qualität möglicher alternativer Routen und Alternativgraphen ist nicht trivial und steht im Fokus der Forschungsgemeinde - zumindest bezüglich der Routenplanung in Straßennetzen. Entsprechende Qualitätsmetriken zur Verwendung außerhalb von Straßennetzen, also beispielsweise für die Fußgängernavigation in Gebäuden, für die maritime Navigation innerhalb von Hafenanlagen oder für die Flugzeugnavigation, fehlen jedoch. In der Veröffentlichung [76], welche die Grundlage für diesen Abschnitt darstellt, wurde versucht, diese Lücke zu schließen. Im Folgenden werden die bereits bestehenden Arbeiten bezüglich Qualitätsmetriken in Straßennetzen gesammelt und strukturiert vorgestellt. Dies wird anschließend im Abschnitt 3.5 dazu verwendet, die Herausforderungen für die Verwendung solcher Qualitätsmetriken im Umfeld komplexer Umgebungen zu untersuchen (siehe die in Abschnitt 3.1 formulierte vierte Herausforderung). Ferner werden mögliche Herangehensweisen für die Übertragbarkeit der Metriken diskutiert sowie die entstehenden Chancen und Grenzen. Ziel der Vorveröffentlichung und somit auch der angesprochenen Abschnitte ist es, die Diskussion innerhalb der Forschungsgemeinde bezüglich Qualitätsmetriken und entsprechenden Zielfunktionen in komplexen Umgebungen zu stimulieren. 


\subsubsection{Qualitätsmetriken für alternative Routen}

Die zentrale Referenz für Qualitätsmetriken von Alternativrouten in Straßennetzen ist [2], eine Publikation, die ihre Vorarbeit [1] durch verschiedene Erweiterungen und einer aufwendigeren Evaluation fortführt. Die Autoren definieren einen Pfad als Kandidaten für eine alternative Route (,admissible path“, etwa zulässiger Pfad), wenn dieser substantiell anders als der Referenzpfad, nicht viel länger und zudem ohne unnötige Umwege ist. Die soeben genannten drei Eigenschaften wurden in [2] zwar formal definiert, werden im Folgenden aber zum besseren Verständnis zunächst informell beschrieben:

1. Limited Sharing: Der alternative Pfad muss substantiell anders als der Referenzpfad sein. Das bedeutet, dass die Gesamtlänge der Kanten, die beide gemeinsam haben, nur ein kleiner Anteil der Länge der Referenzroute sein darf.

2. Local Optimality: Der alternative Pfad darf keine unnötigen Umwege besitzen, und jede lokale Entscheidung muss Sinn machen. Lokale Optimalität ist gegeben, wenn jeder Teilpfad bis zu einer gewissen Länge ein kürzester Pfad ist.

3. Uniformly Bounded Stretch: Der alternative Pfad darf nicht viel länger als der Referenzpfad sein. Die Bedingung der ,guten Dehnung“ wertet zudem die Eigenschaft der lokalen Optimalität auf, da der Fall auftreten kann, dass ein Pfad eine hohe lokale Optimalität besitzt, eine Abkürzung jedoch einen unnötigen Teil der Route auslassen würde.

Basierend auf dieser informellen Beschreibung haben Abraham et al. in [2] nun folgende formale Beschreibung für admissible alternative paths definiert. Sei $G=(V, E)$ ein gerichteter Graph mit nicht-negativen Kantengewichten, wobei $|V|=n$ der Anzahl der Knoten und $|E|=m$ der Anzahl der Kanten entspricht. Gegeben ein Pfad $P$ in $G$, so entspricht $|P|$ der Anzahl der Kanten des Pfades und $l(P)$ der Summe der Kantengewichte. Darüber hinaus ist $l(P \cap Q)$ die Summe der Kantengewichte, die sich die Pfade $P$ und $Q$ teilen und $l(P \backslash Q)$ ist $l(P)-l(P \cap Q)$, also die Summe der Kantengewichte von $\mathrm{P}$, die $\mathrm{P}$ exklusiv begeht. Sind zwei Knoten $s$ und $t$ gegeben, so wird die Suche nach dem kürzesten Pfad - ausgedrückt durch Opt $(s, t=\mathrm{Opt})-$ das Punkt-zu-Punkt-Kürzeste-Pfad-Problem genannt. Gegeben die drei Abstimmungsparameter $0<\alpha<1, \epsilon \geq 0$ und $0 \leq \gamma \leq 1$ sowie ein kürzester Pfad Opt zwischen $s$ und $t$, so ist der $s$-t-Pfad $P$ eine zulässige Alternative (admissible alternative path), wenn die folgenden Kriterien erfüllt werden:

1. Limited Sharing: $l(\mathrm{Opt} \cap P) \leq \gamma \cdot l(\mathrm{Opt})$

2. Local Optimality: $P$ ist $T$-lokal optimal für $T=\alpha \cdot l(\mathrm{Opt})$. Ein Pfad $P$ ist $T$-lokal optimal, wenn jeder Teilpfad $P^{\prime}$ von $P$ mit $l\left(P^{\prime}\right) \leq T$ ein kürzester Pfad ist. 
3. Uniformly Bounded Stretch (UBS): $P$ ist $(1+\epsilon)$-UBS. Ein Pfad $P$ besitzt $(1+\epsilon)$-UBS, wenn für jeden Teilpfad $P^{\prime}$ von $P$ mit Start- und Endpunkten $s^{\prime}, t^{\prime}$, die Ungleichung $l\left(P^{\prime}\right) \leq(1+\epsilon) \cdot l\left(\operatorname{Opt}\left(s^{\prime}, t^{\prime}\right)\right)$ gilt.

In Straßennetzen existiert eine Vielzahl an Möglichkeiten, zwei Punkte zu verbinden. Dies führt dazu, dass selbst die Menge der zulässigen Pfaden oft zu groß ist. Aus diesem Grund haben Abraham et al. eine Untermenge der Klasse der admissible alternative paths definiert, nämlich die single via paths (siehe auch Abschnitt 3.3.1): Sind ein Start $s$, ein Ziel $t$ sowie ein Via-Knoten $v$ gegeben, so ist ein Via-Pfad $P_{v}$ die Konkatenation des kürzesten Pfades von $s$ nach $v$ mit dem kürzesten Pfad von $v$ nach $t$. Diese Definition ist eine unnötige Einschränkung, wie Bader et al. in [10] ausführen, jedoch besitzen Routen solcher Art interessante Eigenschaften. Zum einen besitzt $P_{v}$ die geringste Dehnung unter allen Routen, die durch den Knoten $v$ führen. Dies ist der Tatsache geschuldet, dass $P_{v}$ eine Konkatenation von zwei kürzesten Pfaden ist. Zum anderen kann die Eigenschaft der lokalen Optimalität nur im Umfeld des Knoten $v$ verletzt werden. Diese Tatsache wird unter anderem für die weitere Verbesserung der genannten Kriterien verwendet [2].

Die beschriebenen Kriterien werden als Parameter für eine in [2] nicht weiter definierte Zielfunktion $f(\cdot)$ verwendet. Mittels dieser Funktion können Kandidaten für alternative Routen sortiert und der höchstplatzierte zulässige Pfad kann zurück gegeben werden. Eine naheliegende Parametrisierung der Zielfunktion kann sein, Routen mit niedriger Überlappung (limited sharing), hoher lokaler Optimalität (local optimality) und geringer Länge (uniformly bounded stretch) zu finden. Vorteilhaft bei der Berechnung von mehreren alternativen Routen ist die Tatsache, dass lediglich limited sharing bezüglich des Referenzpfades und aller bisher gefundener alternativer Routen berechnet werden muss. Sowohl local optimality als auch uniformly bounded stretch beziehen sich nur auf den jeweils betrachteten Pfad.

Die Autoren Luxen und Schieferdecker präsentieren in [136] eine Erweiterung der Arbeit von Abraham et al. [2], in der sie die Anfragezeiten optimieren. Die Autoren schlagen einen Algorithmus vor, der eine kleine Menge von vordefinierten Via-Knoten für Paare von Regionen innerhalb des Graphen speichert. Anders ausgedrückt bedeutet dies, dass sie sich auf eine kleine Menge von Kandidaten für admissible alternativ paths fokussieren, die effizient und daher vollständig getestet werden kann. Hierfür verwenden die Autoren Contraction Hierarchies [13], [90] (siehe auch Abschnitt 2.2.2 mit Erweiterungen für das Lösen des Kürzester-Pfad-Problems) und erreichen somit nach eigenen Angaben Routen mit höherer Qualität bei kürzeren Anfragezeiten unter vernachlässigbaren Mehrkosten für den Speicher.

Eine weitere Verbesserung des Ansatzes wird in [124] vorgestellt. Kobitzsch motiviert seinen Beitrag mit der These, dass der Auswahlprozess des in [2] vorgestellten Via-Knoten-Algorithmus aufgrund des Testens der potentiellen Kandidaten und der damit verbundenen zahlreichen Anfragen nach dem kürzesten Pfad zu kostenintensiv ist. Darüber hinaus würden Algorithmen, die 
Kandidaten mittels einer Heuristik ermitteln, bewerten und sortieren, potentiell bessere Kandidaten verwerfen. Der Autor verwendet demnach einen völlig anderen Ansatz, indem er den Test eines Kandidaten (viability check) derart schnell macht, dass alle Pfade getestet werden können. Dies wird dadurch erreicht, dass das vorliegende Problem auf einen kleinen Graphen reduziert wird, der alle potentiell brauchbaren alternativen Routen enthält.

\subsubsection{Qualitätsmetriken für Alternativgraphen}

Die zentrale Referenz für Qualitätsmetriken von Alternativgraphen in Straßennetzen ist [10], eine Veröffentlichung, die auf der Masterarbeit von Dees [44] basiert. Neben diesen Arbeiten wurden vorläufige Ergebnisse bereits in [45] publiziert. Das Hauptkonzept von Alternativgraphen ist die Berechnung einer Menge von Alternativrouten, die grundsätzlich gemeinsame Knoten und Kanten besitzen und deren Teilpfade potentiell zu neuen alternativen Routen kombiniert werden können. Aus diesem Grund motivieren und definieren Bader et al. in [10] einen Alternativgraphen als die Vereinigung mehrerer Pfade mit gleichem Start und Ziel zur kompakten Repräsentation mehrerer Alternativrouten. Die Autoren definieren drei Attribute zur quantitativen Beschreibung der Qualität eines Alternativgraphen und zeigen, dass allein die Optimierung einer einfachen Zielfunktion, die nur zwei der drei vorgestellten Attribute verwendet, bereits NP-schwer ist. Aus diesem Grunde beschreiben Bader et al. entsprechende Heuristiken. Die Maße zur quantitativen Beschreibung der Qualität eines Alternativgraphen werden im Folgenden zunächst informell beschrieben:

1. Total Distance: Dieses Maß beschreibt den Grad, zu dem sich die durch den Alternativgraphen definierten Routen nicht überlappen. Der Maximalwert wird erreicht, wenn der Alternativgraph ausschließlich aus disjunkten Pfaden besteht. Die Ausgestaltung dieses Maßes benötigt eine Skalierung, da andernfalls lange, nicht-optimale Pfade bevorzugt werden würden.

2. Average Distance: Dieses Maß beschreibt die Qualität der im Alternativgraphen enthaltenen Pfade als die durchschnittliche Dehnung der Pfade. Die Ausgestaltung dieses Maßes benötigt eine Mittelung, da andernfalls eine große Anzahl an Pfaden, die alle ähnlich zueinander sind, bevorzugt werden würden.

3. Decision Edges: Dieses Maß beschreibt die Komplexität des Alternativgraphen und wird vornehmlich dafür verwendet, eine für den menschlichen Anwender einfache Repräsentation zu erhalten.

Neben der informellen Beschreibung der Maße haben Bader et al. in [10] folgende formale Definitionen präsentiert. Sei $G=(V, E)$ ein Graph mit einer Kantengewichtsfunktion $w: E \rightarrow \mathbb{R}_{+}$. Gegeben ein Startknoten $s$ und ein Zielknoten $t$, so ist ein Alternativgraph $H=\left(V^{\prime}, E^{\prime}\right)$ ein Graph mit $V^{\prime} \subseteq V$, 
sodass für jede Kante $e \in E^{\prime}$ ein einfacher $s$-t-Pfad in $H$ existiert, der $e$ enthält. Für jede Kante $(u, v)$ in $E^{\prime}$ muss ein Pfad von $u$ nach $v$ in $G$ existieren und das Kantengewicht $w(u, v)$ muss identisch zum Gewicht des Pfades sein. $d_{G}(u, v)$ beschreibt die Distanz des kürzesten Pfades von $u$ nach $v$ in $G$, analog dazu $d_{H}(u, v)$ die Distanz des kürzesten Pfades von $u$ nach $v$ in $H$. Basierend darauf lautet die formale Definition der Qualitätsmetriken für einen Alternativgraphen $H=\left(V^{\prime}, E^{\prime}\right)$ in einem Straßennetz:

$$
\begin{gathered}
\text { Total Distance : } \sum_{e=(u, v) \in E^{\prime}} \frac{w(e)}{d_{H}(s, u)+w(e)+d_{H}(v, t)} \\
\text { Average Distance }: \frac{\sum_{e \in E^{\prime}} w(e)}{d_{G}(s, t) \cdot \text { totalDistance }}
\end{gathered}
$$

$$
\text { Decision Edges : } \sum_{v \in V^{\prime} \backslash\{t\}} \operatorname{outdegree}(v)-1
$$

Wie weiter oben angesprochen ist die Optimierung der drei beschriebenen Maße derart komplex, dass Bader et al. in [10] Heuristiken verwendet, um einen hochqualitativen Alternativgraphen zu erstellen. Hierzu berechnen sie im ersten Schritt den kürzesten Pfad vom Start zum Ziel und fügen diesen in den Alternativgraphen ein. Anschließend berechnen sie schrittweise weitere alternative Pfade und fügen diese in den Alternativgraphen ein, sofern es das Ergebnis der Zielfunktion erhöht (Greedy-Algorithmus oder ,gieriger Algorithmus").

Basierend auf der vorgestellten Idee existieren verschiedene Erweiterungen. In einer Bachelorarbeit [152] wird eine effiziente Implementierung des Konzepts von Bader et al. vorgestellt, die ein Multi-Level-Dijkstra [48] mit der PenaltyMethode kombiniert, welche wiederum um eine Pfadanalyse erweitert wurde.

Kobitzsch et al. präsentieren in [125] eine praxistaugliche und interaktiv verwendbare Implementierung von [10]. Die Autoren modifizieren den PenaltyAlgorithmus durch eine Multi-Level-Partitionierung zusammen mit einem Bestrafungsschema für die Routen und deren angrenzende Kanten. Darüber hinaus wird die Funktion zum Testen der Verwendbarkeit eines Pfadkandidaten abgeändert. Weitere Beschleunigungen werden mittels Customizable Route Planning [48], [52] und Dynamic Level Selection durchgeführt. Die Autoren beschreiben, dass der ursprüngliche Ansatz aus [10] erst bis zu 20 Pfade berechnet, um anschließend eine prioritätsbasierte Auswahl durchzuführen. Kobitzsch et al. hingegen fokussieren sich auf eine geringe Anfragezeit, weshalb sie einen anderen Ansatz verfolgen: sie berücksichtigen unmittelbar den potentiellen Mehrwert eines Pfades für den Alternativgraphen, was bedeutet, dass zum einen der Pfad eine Abweichung einer gewissen Mindestlänge besitzen muss und zum anderen, dass die Umwege bezüglich ihrer Dehnung untersucht werden.

Zeitgleich zur zentralen Referenz wurde in [147] ein Algorithmus veröffent- 
licht, der nach Aussage der Autoren Alternativgraphen mit höherer Qualität erstellt. Paraskevopoulos und Zaroliagis schlagen zuerst einen Vorverarbeitungsschritt vor, der den potentiellen Suchraum reduziert und welcher der heuristischen Methode zum Finden von alternativen Pfaden vorgelagert ist. Anschließend führen sie einen Filter sowie eine Feinabstimmung sowohl für den Plateau-Algorithmus als auch für den Penalty-Algorithmus ein.

\section{5 Übertragbarkeit auf alternative Routen in Freiflächen}

Im vorigen Abschnitt wurden bestehende Arbeiten bezüglich Qualitätsmetriken für Alternativrouten und -graphen in Straßennetzen strukturiert vorgestellt. Abraham et al. beschreiben in [2], dass eine angemessene alternative Route möglichst unterschiedlich zur Referenzroute sein (limited sharing), keine unnötigen Umwege haben (local optimality) und nicht viel länger als die Referenzroute sein sollte (uniformly bounded stretch). Bader et al. schlagen in [10] vor, dass ein guter Alternativgraph Routen beinhalten sollte, die sich wenig überlappen (total distance), eine geringe Dehnung besitzen (average distance) sowie wenig komplex sind (decision edges).

An dieser Stelle bezieht sich die vorliegende Arbeit das letzte Mal auf den Unterschied zwischen Straßennetzen und komplexen Umgebungen. Die Thematik der alternativen Routen ist stark subjektiv, stellenweise philosophisch und erscheint durch die vorhandene Literatur im Kontext der Straßennetze als gelöst, was aber im Kontext von komplexen Umgebungen nicht der Fall ist.

In den folgenden Abschnitten wird nun die Problematik diskutiert, warum die bestehenden Qualitätsmetriken nicht unverändert in komplexen Umgebungen angewendet werden können. Es werden mögliche Herangehensweisen für die Übertragbarkeit diskutiert sowie Chancen und Grenzen beschrieben. Dabei ist das Ziel nicht die abschließende Beantwortung der Problematik, sondern die Anregung zur Diskussion und Entwicklung von Qualitätsmetriken für Alternativrouten und -graphen in besagten komplexen Umgebungen. Der Abschnitt nimmt also Bezug auf die vierte und letzte Herausforderung dieses Kapitels, die in Abschnitt 3.1 beschrieben wurden.

Während der strukturierten Darstellung der bestehenden sechs Qualitätsmaße wird ersichtlich, dass sich einige stark ähneln. Aus diesem Grund werden im weiteren Verlauf limited sharing und total distance zusammen behandelt, da sie beide die Menge der Kanten, die sich die untersuchten Pfade teilen dürfen, behandeln. Ähnlich verhält es sich mit uniformly bounded stretch und average distance, die beide auf der Idee beruhen, dass die Pfade nicht übermäßig lang sein dürfen. Darüber hinaus wird das Maß local optimality diskutiert, das besagt, dass selbst Teilpfade kurz sein müssen. Schließlich ist decision edges ein verbleibendes Konzept der bestehenden Literatur, das für komplexe Umgebungen angepasst werden muss. 


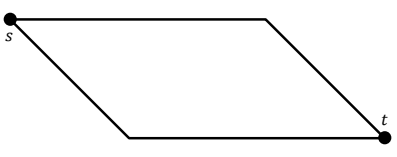

(a)

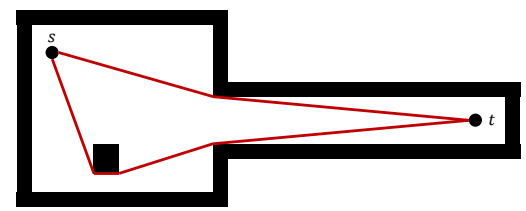

(b)

Abbildung 3.11: Zwei Beispiele, die das Problem von nicht-überlappenden Routen aufgrund von Details der Implementierung darstellen.

\subsubsection{Limited Sharing und Total Distance}

Die zentrale Idee hinter den zwei Maßen ist die, dass sich eine alternative Route wesentlich vom Referenzpfad unterscheiden sollte. Technisch gesehen drücken beide Maße dies über die Länge der Teilpfade aus, die bei den untersuchten Routen identisch sind. Bei limited sharing wird der Vergleich auf den Referenzpfad und den Kandidaten für eine alternative Route angewendet, bei total distance wird gemessen, ob das Einfügen des Kandidaten die Qualität des Alternativgraphen erhöht. In der Praxis ist es also so, dass die Optimierung des Alternativgraphen bezüglich dieser Metrik abhängig von der Reihenfolge der Kandidaten ist.

Die wichtigste Frage bei der Verwendung der Maße in komplexen Umgebungen ist nun, wie die gemeinsame Verwendung von Teilpfaden in einer eindeutigen Art und Weise definiert werden kann. An dieser Stelle drängt sich der Begriff der Überlappung auf, der sich bei den besagten Maßen in Straßennetzen ausschließlich auf identische Kantenfolgen im Graphen bezieht.

Selbst auf einer sehr grundlegenden Ebene wie der Implementierung eines simplen Kürzester-Pfad-Algorithmus wie etwa dem Algorithmus von Dijkstra fängt die Mehrdeutigkeit an. Der Algorithmus verwendet eine Prioritätenliste, in der alle Knoten enthalten sind. Die Sortierung entspricht, wie bereits in Abschnitt 2.2.2 erläutert, der (vorläufigen) Distanz zum Startknoten und kann im Umfeld komplexer Umgebungen mit vielen gleichlangen Kanten zu mehreren gleichlangen kürzesten Pfaden führen. Die Nichteindeutigkeit kann dazu führen, dass sich der kürzeste Pfad von $s$ nach $t$ vom kürzesten Pfad von $t$ nach $s$ unterscheidet. In Abbildung 3.11 wird dies anhand von zwei vereinfachten Beispielen illustriert. In Abbildung 3.11a ist zu erkennen, dass sich der Weg von $s$ nach $t$ zunächst immer möglichst links hält, das heißt, den linken möglichst kürzesten Pfad wählt und erst spät nach rechts führt. Auch auf dem Weg von $t$ nach $s$ ist dies zu erkennen, weshalb sich die zwei resultierenden Pfade nicht überlappen. In Abbildung 3.11b sind zwei Wege von $s$ nach $t$ dargestellt, wobei einer das kleine Hindernis umläuft und der andere nicht. Auch diese beiden Routen überlappen sich offensichtlich nicht.

Ein weiterer Grund, warum die implizite Definition der Überlappung, wie sie in Straßennetzen konzipiert wurde, in komplexen Umgebungen nicht unverän- 
dert anwendbar ist, liegt in der potentiellen Mehrdeutigkeit äquivalenter Routen mit äquivalenter Bedeutung. In Abbildung 3.2, die bereits zur Erläuterung des Konzepts der Homotopie verwendet wurde, sind vier Routen dargestellt, die sich offensichtlich nicht überlappen. Die Frage, ob die zwei mittleren Routen $q$ und $r$ dadurch tatsächlich gute alternative Routen darstellen, ist jedoch diskussionswürdig. Wenn die zugrunde liegende Karte einen sehr großen Maßstab hätte, sodass keine Interaktion zwischen den Nutzern der beiden Routen zu erwarten wäre, wie es beispielsweise bei der Flugzeugnavigation oder im maritimen Umfeld der Fall sein dürfte, so könnten die zwei Routen als echte Alternativen akzeptiert werden. In einem Szenario mit kleinerem Maßstab, wie der Fußgängernavigation innerhalb eines Gebäudes, bei dem sich die Fußgänger sehen und miteinander kommunizieren können, so können die Routen als äquivalent gelten. Es ist also eine Mehrdeutigkeit vorhanden.

Eine Möglichkeit diese Schwierigkeiten anzugehen ist, eine Vorgehensweise zu definieren, mit der anwendungs- und kartenbezogenen entschieden werden kann, welche Punkte zweier Routen sich tatsächlich „,überlappen“. Im Folgenden werden drei generelle Strategien vorgestellt, wie die Qualitätsmetriken limited sharing und total distance, die sich beide auf die Überlappung von Routen beziehen, auf das Szenario der komplexen Umgebungen ausgeweitet werden können.

\section{Kartendarstellung}

Die Maße für die Qualität von alternativen Routen und Alternativgraphen in Straßennetzen wurden ursprünglich zur direkten Verwendung in Graphen definiert. Dies ist möglich, da die Abbildung einer tatsächlich gefahrenen Route auf den Graphen ebenfalls eindeutig ist. Demnach wäre es für das Szenario der komplexen Umgebungen wünschenswert, wenn alle möglichen Pfade in den frei begehbaren Umgebungen derart konsistent auf einen Graphen abgebildet werden können, dass unterschiedliche Kanten semantisch unterschiedliche Bewegungen bedeuten. Ist dies der Fall, so haben zwei Pfade, die beide eine spezifische semantische Bewegung wie etwa den gemeinsamen Verlauf durch einen Gang besitzen, eine erhöhte Überlappung.

Es existieren viele Ideen für die Erstellung von Navigationsgraphen für spezifische Anwendungsfälle in komplexen Umgebungen (siehe Abschnitt 2.3.2). Dennoch bedeutet die Realisierung eines solchen Navigationsgraphen stets auch einen Kompromiss zwischen der Aussagekraft der Karte und der entstehenden Eindeutigkeit. Wenn beispielsweise ein langer Korridor nur mittels einer einzigen Kante repräsentiert wird, sodass alle Pfade der Passanten überlappen (hohe Eindeutigkeit), so können diese eben auch nur in einer Art und Weise in die Karte eingebettet werden, was aus Sicht der Anwendung unbefriedigend sein kann (niedrige Aussagekraft). Dies ist in Abbildung 3.12a skizziert. Wenn der Graph allerdings eine ausdrucksstärkere Darstellung in der Karte erhalten soll, so steigt auch die Komplexität des Graphen und die eindeutige Überlappung verschwindet (siehe Abbildung 3.12b). 


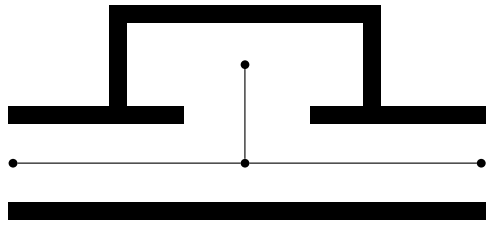

(a)

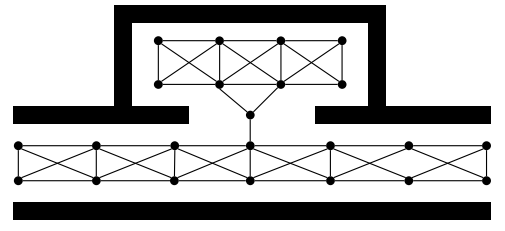

(b)

Abbildung 3.12: Kompromiss zwischen der Aussagekraft einer Karte, der Komplexität und der entstehenden Eindeutigkeit. Je höher die Aussagekraft, desto höher die Komplexität und desto geringer die Eindeutigkeit.

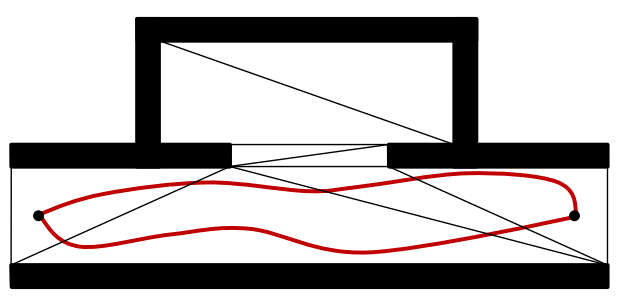

Abbildung 3.13: Zwei Routen durchlaufen dieselben Polygone einer beispielhaften Triangulierung und besitzen somit eine hohe Überlappung.

Für die Techniken zur Erstellung von Navigationsgraphen (siehe Abschnitt 2.3.2) ist die Anzahl der resultierenden Knoten und Kanten abhängig von der Komplexität der involvierten Geometrie (einfache rechteckige Büroräume vs. unstrukturierte Umgebung mit runden Hindernissen). Das bedeutet gleichzeitig, dass auch die Aussagekraft eines möglichen Maßes zur Bestimmung der Überlappung abhängig ist von der involvierten Geometrie.

Zusammengefasst ergibt sich daraus, dass mittels Kartendarstellungen allein keine universelle Definition der Überlappung erreicht werden kann. Wenn die Anwendung jedoch ohnehin eine Technik zur Erstellung eines Navigationsgraphen verwendet, so kann dieser Graph zumindest für eine schwache Abschätzung der Überlappung verwendet werden. Naheliegende Ideen zur Definition einer (möglicherweise mehrdeutigen) Überlappung sind die Analyse der gemeinsam durchlaufenen Polygone, die zum Beispiel durch Triangulierung erzeugt werden können, oder eine identische Sequenz durchquerter Polygonseiten. Abbildung 3.13 zeigt eine beispielhafte Triangulierung sowie zwei Routen, die dieselben Polygone in einer identischen Sequenz der Polygonseiten durchqueren. Dies ist ein Indiz für eine mögliche hohe Überlappung. 


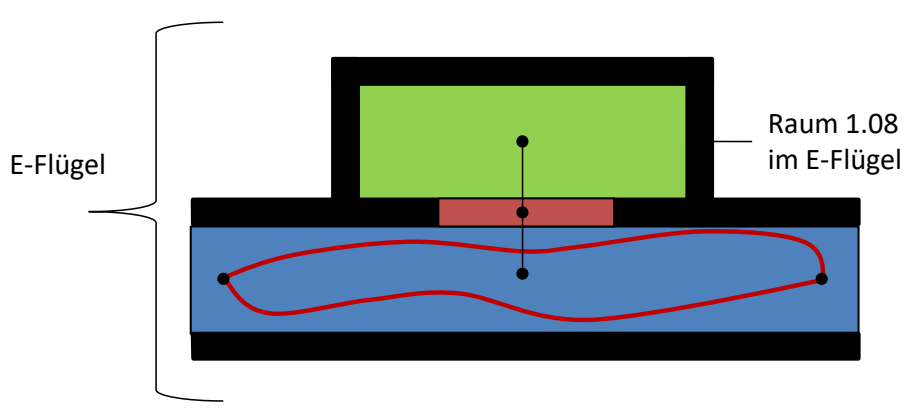

Abbildung 3.14: Überlappung durch gemeinsam durchlaufene zusammenhängende Flächen.

\section{Verbundene Fläche}

Ein zweiter Ansatz zur Definition von Überlappung in komplexen Umgebungen erhöht die betrachtete Dimension und fokussiert damit anstatt Punkte die Fläche. Die Idee ist die Identifizierung zusammenhängender Flächen, die zwei Routen gemeinsam haben. Abstrahiert gesehen bezieht sich diese Denkweise auf die Topologie einer Karte. Ideal wäre eine logische Karte, bei der jeder Punkt genau einer verbundenen Fläche zugeordnet werden kann, was jedoch in der Praxis nicht immer zu erreichen ist. Beispielsweise ist die Fläche unterhalb eines Türrahmens nicht eindeutig einem Raum zuzuordnen, Punkte innerhalb benannter Räume in Gebäuden sind dies hingegen schon. So ist beispielsweise „E 1.08“ ein eindeutiger Raum, der sich in der ersten Etage des E-Flügels befindet. In Abbildung 3.14 haben die eingezeichneten Routen eine hohe Überlappung, da sie gemeinsam die zusammenhängende Fläche mit der Semantik „Gang im E-Flügel“ durchqueren.

Ein weiteres Verständnis von Überlappung, das sich ebenfalls auf die Topologie der Karte bezieht, kann mittels der Homotopie von zwei Routen beschrieben werden (siehe Abschnitt 3.2). Demnach überlappen sich zwei Routen solange, wie sie kontinuierlich ineinander überführbar sind. Diese Idee ist jedoch, wie bereits beschrieben, binär und entscheidet nicht zwischen kleinen Hindernissen und großen Umwegen. Zusätzlich kann das Konzept der Homotopie nur auf Paare von Routen mit gleichem Start und Ziel angewendet werden, wodurch eine „Teilüberlappung“ von Routen nicht messbar ist.

\section{Distanzmaß}

Die Motivation für den dritten Ansatz zur Definition von Überlappung in komplexen Umgebungen ist durch die beschriebenen Nachteile der Homotopie gegeben: die binäre und zählende Natur der ursprünglichen Maße limited sharing und total distance könnten durch eine vollständig kontinuierliche Struktur ersetzt werden. Die Idee dabei ist die Verwendung einer Distanz zwi- 


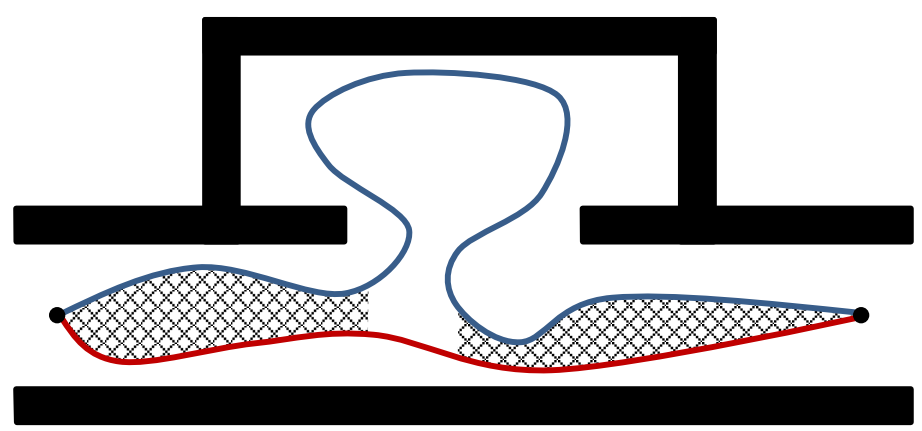

Abbildung 3.15: Überlappung durch kontinuierliche Struktur einer Distanz. Mittels eines Schwellwerts wird zwischen sich überlappenden und nicht-überlappenden Teilpfade unterschieden.

schen den Routen, um darüber eine Überlappung zu definieren (siehe auch die später vorgestellte archetypische Distanz in Abschnitt 4.4). Die Verwendung einer kontinuierlichen Distanz ist in der Hinsicht elegant, als dass die klassischen Graph-basierten Definitionen der Überlappung als ein Sonderfall der Distanz-basierten Definition angesehen werden können, bei denen es nur die Distanz Null (Überlappung) oder von Null verschieden (keine Überlappung) gibt. In einer kontinuierlichen Version kann ein Schwellwert verwendet werden, um zwischen sich überlappenden und nicht-überlappenden Teilpfaden zu unterscheiden. Abbildung 3.15 zeigt ein Beispiel, bei dem sich die zwei Routen am Anfang und am Ende überlappen, in der Mitte hingegen nicht. Natürlich kann die Verwendung eines kontinuierlichen Maßes für die Überlappung bereits in die Generierung von alternativen Routen integriert werden und nicht erst bei der Erstellung eines Alternativgraphen.

\subsubsection{Uniformly Bounded Stretch und Average Distance}

Die grundlegende Idee hinter diesen zwei Qualitätsmetriken ist die, dass eine Alternativroute nicht viel länger als der kürzeste Pfad sein sollte. Die Maße beziehen sich also auf die durchschnittliche Dehnung der untersuchten Route oder des untersuchten Graphen. Bei uniformly bounded stretch bedeutet dies, dass die Dehnung beliebiger Teilpfade einen gewissen Grad nicht überschreiten darf. Die Dehnung wird also insgesamt auf die gesamte Route angewendet. Bei average distance werden die Überlängen der im Alternativgraphen enthaltenen Routen als Ganzes betrachtet und bewertet.

Die Begrenzung der Dehnung eines Pfades ist zunächst eine unkomplizierte Technik, um bei der Erstellung von alternativen Routen die Länge des Umwegs einzuschränken. Wenn der Schwellwert zur Begrenzung zu schwach gewählt ist, so werden zu viele Kandidaten berücksichtigt, und die Effizienz der Algorithmen wird vermindert oder es werden zu viele Ergebnisse zurück 
gegeben, was die Qualität des Algorithmus schwächt. Wenn jedoch die Dehnung zu stark beschränkt wird, so können einige Kandidaten für alternative Routen nicht entdeckt werden. Der ideale Wert für die Dehnung hängt also sowohl von der Anwendung ab (wie viele Alternativen werden im weiteren Verarbeitungsschritt benötigt?) als auch von der behandelten räumlichen Umgebung: An einem Flughafen mögen längere Alternativen annehmbar sein; in der Fertigungstechnik, in der beispielsweise die Bewegung eines Montageroboters berechnet wird, führen Umwege zu längerer Produktionszeit und somit zu steigenden Unkosten.

Zusätzlich ist zu beachten, dass sich im Umfeld der komplexen Umgebungen die Länge einer Route in einer Kartendarstellung stark von der tatsächlichen Länge in der Realität unterscheiden kann (siehe auch Abschnitt 2.3.2). Beispielsweise werden in navigation meshes oft die zentralen Punkte oder die Kanten größerer Polygone als Wegpunkte verwendet. Dies kann dazu führen, dass die Routen in der Kartendarstellung länger sind als in Wirklichkeit. In Straßennetzen ist der Zusammenhang zwischen tatsächlich gefahrener Strecke und entsprechend im Graphen durchlaufenen Knoten und Kanten wesentlich stärker.

Schließlich ist, zumindest für das Dehnungsmaß bei der Berechnung einzelner alternativer Routen, zu klären, was mit „Teilpfad“ gemeint ist. Während im Kontext von Straßennetzen ein Teilpfad eindeutig als Kantenzug definiert ist, also jeweils an einem Knoten beginnt und endet, so ist dies im Kontext komplexer Umgebungen erneut mehrdeutig. Die exakte Ausgestaltung eines Teilpfades kann je nach Kartendarstellungen stark unterschiedlich ausfallen, von sehr fein (vollvermaschtes Belegungsraster) bis sehr grob (navigation mesh).

Zusammengefasst lässt sich sagen, dass auch diese zwei Maße erneut eine starke Abhängigkeit von der Kartenrepräsentation und dem Anwendungsfall besitzen.

\subsubsection{Local Optimality}

Das Kriterium der lokalen Optimalität besagt, dass eine alternative Route keine unnötigen Umwege besitzen darf. In [2] wurde dieses Maß eingeführt mit der Definition, dass alle Teilpfade bis zu einer bestimmten Länge kürzeste Pfade sein müssen. Im Kontext der Veröffentlichung von Abraham et al. ist dies eine wichtige Eigenschaft, da die Autoren alternative Routen durch die Konkatenation von zwei kürzesten Pfaden erstellen, was dazu führt, dass der kombinierte Pfad extreme Wendepunkte besitzen kann. Um diesem Effekt entgegenzuwirken wird der Kontaktpunkt der zwei kürzesten Pfade überprüft und ermittelt, ob eine sinnvolle Abkürzung existiert. Auch bei der Berechnung von Alternativgraphen wurde dieses Problem identifiziert, jedoch haben die Autoren die entsprechende Lösung in einen Nachbearbeitungsschritt verschoben [10]. Dies ist durchaus ein gangbarer Weg, da durch eine ungeschickte Verwendung von im Alternativgraphen enthaltenen Teilpfaden übermäßig lan- 
ge alternative Routen erstellt werden können, was die Notwendigkeit für eine Nachbearbeitung ohnehin impliziert.

Während die lokale Optimalität im Graphen eines Straßennetzes scharf definiert ist und behandelt werden kann, ist sie im Umfeld der komplexen Umgebungen nicht eindeutig. Zum einen stellt sich die Frage, wie Umwege behandelt werden, die nur aufgrund von Ungenauigkeiten in der Übersetzung von Karte zu Graph entstehen. Zum anderen resultiert aus der potentiell enormen Menge von Kanten (beispielsweise beim Belegungsraster), dass das Testen der lokalen Optimalität einen hohen Berechnungsaufwand besitzt. Dies liegt daran, dass die ursprüngliche Definition von lokaler Optimalität jeden Teilpfad bis zu einer bestimmten Länge überprüft. Anders als in Straßennetzen, in denen eine Kante einen möglicherweise langen Straßenabschnitt repräsentiert, kann es sein, dass je nach Kartendarstellung eine Kante im Graphen einer komplexen Umgebung nur eine geringe reale Länge besitzt. Es existieren also potentiell sehr viele solcher zu testenden Teilpfade.

Des Weiteren müsste in diesem Zusammenhang diskutiert werden, ob die Interpretation von lokaler Optimalität für Routen in komplexen Umgebungen folgendermaßen erweitert werden muss: nicht eine möglichst kurze Alternativroute soll gefunden werden, sondern eine möglichst einfache. Denn in den meisten Fällen wird die Suche nach möglichst kurzen alternativen Routen innerhalb komplexer Umgebungen konträr zu den Zielen der Anwendungsebene sein, da kürzeste Pfade oft ein unnatürliches Aussehen besitzen, wie etwa der Verlauf nah an der Gebäudegeometrie oder das Überqueren von Räumen in Diagonalen. Wenn tatsächlich bei der Optimierung einer Route die Länge von Interesse ist, so existieren zahlreiche Forschungsarbeiten bezüglich der Vereinfachung von Trajektorien, was entsprechend in dieser Situation angewendet werden kann [187].

Schließlich stellt sich die Frage, wann ein Umweg unnötig und wann notwendig ist. In einer komplexen Umgebung kann gerade das Begehen eines scheinbaren Umweges die Erstellung einer alternative Route erwirken. Dies kann unter Umständen wieder „nur“ auf einer semantischen Ebene stattfinden, was durch die ursprünglichen graphenbezogenen Maße nicht abgebildet werden kann.

Die diskutierten Herausforderungen können im Grunde auf die Frage reduziert werden, was genau ein „optimaler“ Pfad ist. Es bietet sich an, die Ermittlung eines solchen optimalen Pfades nachzulagern. Im Kontext von komplexen Umgebungen sollte, wie auch in der Vorveröffentlichung [76] erwähnt, die lokale Optimierung als ein anwendungsabhängiger Nachbearbeitungsschritt angesehen werden, da ansonsten die Bedeutung von „optimal“ unklar ist.

\subsubsection{Decision Edges}

Das Qualitätskriterium der Entscheidungskanten (decision edges) besagt, dass ein Alternativgraph eine geringe Komplexität besitzen soll. Hierzu wird in der ursprünglichen Definition die Anzahl der Entscheidungsmöglichkeiten an den 


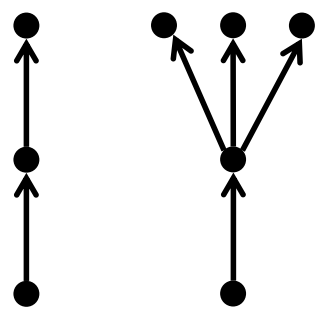

(a) Eine Entscheidung ist ab einem Ausgangsgrad größer 1 möglich.

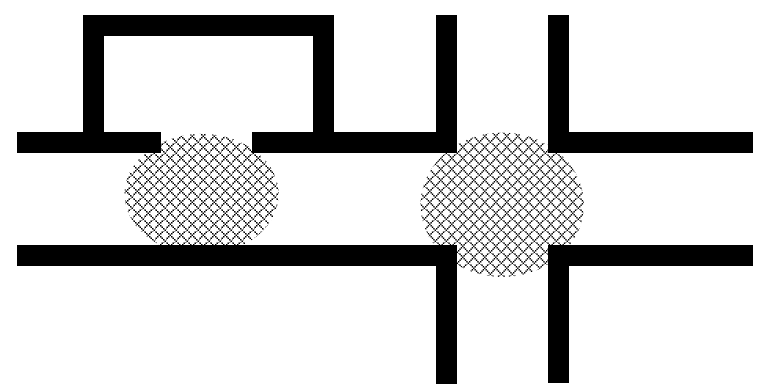

(b) Schematische Darstellung von Kreuzungen, bei denen die schraffierte Fläche Orte für eine Entscheidung darstellen kann.

Abbildung 3.16: Entscheidungen finden in komplexen Umgebungen eher in Flächen als an Punkten statt.

Knoten gezählt.

Ein Knoten mit nur einer ausgehenden Kante besitzt keine Entscheidungskante, da ein Algorithmus entsprechend keine Auswahl neben der einen ausgehenden Kante hat (siehe Abbildung 3.16a). Ein Knoten mit beispielsweise drei ausgehenden Kanten hat hingegen zwei Entscheidungskanten, da neben einer obligatorischen Kante zwei Alternativen existieren. Die Anzahl der Entscheidungskanten eines Alternativgraphen setzt sich somit aus der Summe der Entscheidungskanten aller enthaltenen Knoten zusammen. Das Ziel bei der Erstellung eines Alternativgraphen ist es, den Wert der Entscheidungskanten gering zu halten, sodass aus dem Graphen nur eine kleine Menge von Alternativrouten erstellt werden kann. Wenn der Schwellwert jedoch zu restriktiv gewählt ist, so werden erneut zu viele potentiell gute Alternativen verworfen.

Die Übertragung dieses Maßes von Straßennetzen in komplexe Umgebungen ist ähnlich zur Diskussion der Überlappung (siehe Abschnitt 3.5.1). Im Grunde kann sogar das Verständnis für eine Entscheidung in komplexen Umgebungen mittels Überlappung definiert werden: Eine Entscheidung findet genau dann statt, wenn sich der Zustand der Überlappung ändert. Gewissermaßen wird somit die Idee der Überlappung von Kanten auf die Überlappung von Knoten transferiert.

Während in Straßennetzen eindeutig ist, dass jede Abbiegung eine Entscheidung bedeutet, so ist eine solche isolierte Entscheidung bei der Fußgängernavigation (auch im maritimen Umfeld oder der Luftfahrt) nicht eindeutig. Viel mehr findet eine Entscheidung innerhalb einer Fläche und nicht an einem Punkt statt.

Eine mögliche Annäherung an eine Definition für Entscheidung in komplexen Umgebungen kann daher in den Erstellungsprozess einer Karte integriert werden. Beispielsweise könnte innerhalb eines Gebäudes die Fläche einer Kreuzung markiert sein (siehe Abbildung 3.16b). Eine Entscheidung wird innerhalb 
einer solchen Fläche getroffen und zwar dann, wenn der Pfad die Fläche verlässt. Zwei Routen werden dann an besagter Fläche eine Entscheidung besitzen, wenn sie beispielsweise die Kreuzung durch die gleiche Seite der Fläche betreten, jedoch durch verschiedene Seiten verlassen.

\subsubsection{Gegenseitige Beeinflussung der Qualitätsmetriken}

In den vorigen Abschnitten wurde diskutiert, welche Herausforderungen und möglichen Herangehensweisen bei der Übertragung der Qualitätsmetriken für alternative Routen und Alternativgraphen in komplexen Umgebungen bestehen. Bei der Diskussion wurde schnell ersichtlich, dass nicht nur ein starker Einfluss der gegebenen Anwendung sowie der Karte, sondern auch eine gegenseitige Beeinflussung der Qualitätsmetriken bestehen. Bei dem Versuch, die Maße im Umfeld der komplexen Umgebungen anzuwenden, sollte dies bedacht werden.

Wenn beispielsweise das Konzept der Überlappung verändert wird, so muss sorgfältig abgewägt werden, welche Konsequenzen das für Entscheidungskanten hat - und umgekehrt. Wie weiter oben angesprochen wurde, liegt eine Entscheidung nämlich genau dann vor, wenn sich der Zustand der Überlappung ändert. Das Kriterium der lokalen Optimalität kann indirekt die Form der resultierenden Routen beeinflussen und somit wiederum einen starken Einfluss auf die Überlappung und damit auch auf die Entscheidungskanten haben. Ebenso kann die Optimierung erwirken, dass Routen, die andernfalls von der Dehnung (uniformly bounded stretch und average distance) abgelehnt werden würden, nun doch in einen akzeptablen Wertebereich fallen.

\subsection{Zusammenfassung}

Dieses Kapitel hat sich mit der Berechnung alternativer Routen in Freiflächen befasst. Es wurde ein neuartiger Ansatz vorgestellt, um alternative Routen in Navigationsszenarien wie beispielsweise einem Flughafen zu finden. In Abschnitt 3.2 wurde vorgeschlagen, das topologische Konzept der Homotopie zu verwenden, um zu entscheiden, ob zwei unterschiedliche Pfade als äquivalent oder alternativ anzusehen sind. Ferner wurde ein effizienter Weg beschrieben, mit dem die Äquivalenzrelation schnell angenähert werden kann. Dies wurde mittels eines Polygon-Karten-Schnittes realisiert. Zusammengefasst handelt es sich dabei um die ersten zwei Herausforderungen des Kapitels, wie sie in Abschnitt 3.1 beschrieben wurden.

Zukünftige Arbeiten sind an dieser Stelle eher technischer Natur. So könnte zum Beispiel die Einschränkung, dass die in der vorliegenden Arbeit präsentierte Implementierung lediglich Routen ohne Selbstschnitt als Eingabe verwendet, behoben werden. Zudem kann auch die Berücksichtigung der Windungszahl der Routen bei der weiteren Verarbeitung eine Rolle spielen oder das „Volumen“ 
der Hindernisse, die von zwei Routen umschlossen werden. Schließlich ist eine stapelweise anstatt einer paarweisen Berechnung der Homotopie von Interesse.

In Abschnitt 3.3 wurden Heuristiken zum Finden nicht-homotoper Pfade vorgestellt (dritte Herausforderung aus Abschnitt 3.1). Die soeben beschriebene Definition sowie deren Annäherung wurden verwendet, um die aus der Navigation in Straßennetzen bekannten Algorithmen Penalty und One-Patching für komplexe Umgebungen zu implementieren. Es wurde gezeigt, dass insbesondere der One-Patching-Algorithmus in zeitkritischen Szenarien verwendet werden sollte, wenn die notwendigen Informationen zur Konkatenation von zwei kürzesten Pfaden im Vorfeld berechnet wurden (beispielsweise durch das Lösen des all-pairs-shortest-path problem). Hervorzuheben sind die vorgestellten Nebenprodukte der Heuristiken. Die Gewichtskarte, also die Visualisierung der durch den Penalty-Algorithmus erhöhten Kantengewichte, gibt einen Eindruck in die Struktur der Karte. Auch die Referenzkarte des One-PatchingAlgorithmus, die die entstehenden Homotopieklassen innerhalb einer Karte visualisiert, kann für weitere Analyseschritte verwendet werden.

Bei den resultierenden Routen beider Algorithmen ist eine Notwendigkeit zur Nachbearbeitung zu erkennen. Der Penalty-Algorithmus produziert „rauschende" Pfade insbesondere im Bereich vom Start und Ziel und der OnePatching-Algorithmus erzeugt unter Umständen unnötige Umwege im Bereich des verwendeten Stützpunkts. In beiden Fällen könnte dieses Problem gelöst werden, indem der kürzeste homotope Pfad berechnet und als Repräsentant einer Homotopieklasse verwendet wird. Darüber hinaus könnten adaptive Abbruchkriterien für die Durchführung der Heuristiken erstellt werden. Beispiele hierfür sind die Einbeziehung der Geometrie beziehungsweise der Topologie der Karte sowie die Einbeziehung der Qualität der resultierenden Routen. Auch die Rückgabe eines Alternativgraphen anstatt einzelner Alternativrouten ist denkbar. Ferner könnte das Konzept der homotopen kürzesten Pfade in Navigationssysteme innerhalb von Gebäuden integriert werden: die tiefgreifenden topologischen Informationen können beispielsweise bei der Durchführung von Map Matching hilfreich sein.

Schließslich wurde in den Abschnitten 3.4 und 3.5 die Qualität von alternativen Routen und Alternativgraphen behandelt. Zuerst wurden die unterschiedlichen Konzepte von Qualitätsmetriken im Bereich der Straßennetze strukturiert dargestellt. Es wurde ersichtlich, dass die semantische Entsprechung von Knoten und Kanten mit realen Straßen und Kreuzungen nicht in Kartendarstellungen komplexer Umgebungen vorhanden ist, weshalb die Übertragbarkeit der Maße auf Alternativrouten und -graphen in Freiflächen diskutiert wurde (vierte Herausforderung des Kapitels). Es wurden mögliche Ansätze zur Durchführung beschrieben und insbesondere die Einschränkungen abgewogen. Dabei wurde deutlich, dass die zahlreichen Entscheidungsmöglichkeiten für eine Übertragbarkeit der Metriken miteinander verbunden sind und eine gemeinsame anstatt einer isolierten Betrachtung notwendig ist. Zudem hat die Wahl der Kartendarstellung große Auswirkungen auf die Realisierbarkeit der Maße. Trotz der 
starken Abhängigkeit vom Anwendungsfall und von den Kartendarstellungen ist eine weitere Integration von komplexen Umgebungen in die Forschung der Alternativrouten und -graphen sinnvoll. Ziel sollte die Diskussion sowie Entwicklung universeller Wege zur Erstellung und Auswahl von Alternativrouten sein. Insbesondere die zu erwartende Zusammenführung komplizierter Mobilitätsmodi in die intermodale Navigation - beispielsweise die Kombination von Fußgängernavigation mit Leihfahrrädern und mobilen Robotern in Produktionsszenarien - zeugt von der Notwendigkeit einer integrierten Behandlung von geografischer Umgebung und Berechnung von Pfaden. 


\section{Vergleich geospatialer Trajektorien}

Im vorigen Kapitel wurden alternative Routen in komplexen Umgebungen definiert und Heuristiken vorgestellt, mit denen (potentiell viele) solche Routen erstellt werden können. Basierend auf der binär arbeitenden Homotopie - zwei Routen sind entweder in der gleichen Homotopieklasse oder nicht - kann je nach Struktur der zu analysierenden Umgebungen eine Vielzahl von Pfaden berechnet werden, die sich nur durch kleine Variationen unterscheiden. Außerdem wurde im vorigen Kapitel erläutert, warum bestehende Qualitätsmetriken für alternative Routen in Straßennetzen zur Generierung von alternativen Routen und zur Erstellung von Alternativgraphen im Kontext von komplexen Umgebungen nicht ohne weiteres anzuwenden sind. In dem vorliegenden Kapitel werden nun Ansätze vorgestellt, mit denen geospatiale Trajektorien zeitliche geordnete Punkte im Raum, wie etwa Pfade innerhalb eines Gebäudes - verglichen werden können. Grundsätzliches Ziel dieser Ansätze ist das Verringern einer potentiell hohen Anzahl von Routen, sodass eine möglichst kleine Menge möglichst unterschiedlicher Routen erhalten bleibt.

Dazu wird in Abschnitt 4.3 ein Nebenprodukt der Berechnung alternativer Routen verwendet, um für eine gegebene Menge von Routen eine Rangfolge zu erstellen. Die Routen werden anhand einer Überlastungswahrscheinlichkeit bewertet, sodass je nach Anwendung potentiell stark oder niedrig frequentierte Pfade zurückgegeben werden.

Anschließend wird die archetypische Analyse, ein sogenanntes fuzzy Clusteringverfahren, dazu verwendet, eine gegebene Menge von Routen zu gruppieren und je Gruppe einen Repräsentanten auszuwählen (Abschnitt 4.4.1). Hierdurch wird die Zahl der Routen ebenfalls stark verringert und es bleibt nur eine im Vorfeld definierte Anzahl an unterschiedlichen Routen über. Im Zuge dieser Betrachtung wurden nicht nur archetypische Routen definiert, sondern es wurde auch das Konzept der archetypischen Distanz (Abschnitt 4.4.2) entwickelt. Mittels dieses neuartigen Distanzmaßes können nicht nur bestehende Routen miteinander verglichen werden, sondern auch solche, die neu zu einem bestehenden Datensatz hinzu kommen ohne diese mit den bestehenden Routen explizit vergleichen zu müssen.

Da Clusteringverfahren stets eine wie auch immer geartete Form einer Ähnlichkeit beziehungsweise Distanz verwenden, wird im vorliegenden Kapitel mit der Einführung von archetypischen Routen und der archetypischen Distanz eine nichtbinäre Beschreibung von alternativen Routen vorgestellt. 


\subsection{Motivation und Herausforderungen}

Im vorigen Kapitel wurde eine Definition von alternativen Routen in komplexen Umgebungen basierend auf dem topologischen Konzept der Homotopie gegeben. Zwei Routen mit denselben Start- und Zielpunkten sind äquivalent zueinander, wenn die von ihnen aufgespannte Fläche keine Hindernisse beinhaltet. Der Vorteil dieses Ansatzes ist die einfache und klare Definition zusammen mit der Tatsache, dass diese Definition eine Äquivalenzrelation darstellt. Der große Nachteil ist jedoch der, dass recht schnell eine große Menge alternativer Routen gefunden wird, die dieser Definition gerecht werden. So verursacht ein Gebäudeplan, in dem kleine Gegenstände oder Möbel eingezeichnet sind, die Identifizierung von sehr vielen alternativen Routen, selbst wenn die Routen nur kleine Variationen besitzen. Von der Anwendungsebene her gesehen bietet der Ansatz also potentiell zu viele Alternativen, von denen die wichtigsten Kandidaten erst noch ausgewählt werden müssen.

Ein mögliches Kriterium für die Abschätzung der Wichtigkeit einer Route ist die Wahrscheinlichkeit einer Überlastung. Eine Überlastung ist - vereinfacht ausgedrückt - genau dann gegeben, wenn der Verkehr an einem bestimmten Punkt oder an einem Segment stärker ist als die tatsächliche Kapazität. Im Straßenverkehr ist dies an zentralen Autobahnen zu beobachten, in komplexen Umgebungen wie etwa innerhalb von Gebäuden an engen Gängen oder Türen. In vielen Anwendungsfällen werden Routen bevorzugt, die potentiell verkehrsarm sind, also solche, die überlastete Stellen oder Segmente möglichst vermeiden. Ein Beispiel hierfür ist ein Navigationssystem an einem Flughafen, das dem Anwender eine komfortable und nicht überlastete Route präsentieren möchte. Andererseits existieren Anwendungsfälle, bei denen potentiell verkehrsreiche Flächen bevorzugt werden. Ein beispielhaftes Szenario, bei dem solches Wissen verwendet werden kann, ist die Platzierung von Werbung, da Werbetreibende versuchen, Anzeigen möglichst an hoch frequentierten Stellen zu platzieren.

In Abschnitt 4.3 wird die Bewertung und Einordnung von geospatialen Trajektorien - insbesondere von alternativen Routen im Umfeld komplexer Umgebungen - behandelt, wobei die zugrundeliegende Bewertungsfunktion implizit erstellte Informationen bezüglich der Topologie des Gebäudeplans besitzt. Es wird der Begriff der Überlastungswahrscheinlichkeit vorgeschlagen, um ein erstes Verständnis für die Einschätzung der Wichtigkeit von alternativen Routen innerhalb von Gebäuden zu erhalten und um diese zu bewerten. Die grundlegende Idee hinter dem Algorithmus ist die Annahme, dass Punkte innerhalb einer Karte, die häufig auf einem kürzesten Pfad liegen, ebenfalls häufig durchlaufen werden.

Auch im zweiten Teil des Kapitels wird die Tatsache behandelt, dass die Definition von alternativen Routen mittels Homotopie je nach Gebäudeplan eine große Menge von Routen mit potentiell kleinen Variationen zurückgibt. Abschnitt 4.4 konzentriert sich auf die Frage, wie eine kleine Menge alternati- 
ver Routen mit paarweise sinnvollem Unterschied aus einer gegebenen Menge von (alternativen) Routen zwischen zwei Punkten extrahiert werden kann. Der Ansatz verwendet das Konzept der archetypischen Analyse [36] - eine statistische Methode zur Analyse multivariater Datensätze - und soll als Nachbearbeitungsschritt zum besseren Verständnis von gegebenen alternativen Routen dienen. Das Ziel des Vorgehens ist die Identifizierung einer kleinen Teilmenge von „reinen Exemplaren“, sogenannter Archetypen. Archetypen repräsentieren ideale Beobachtungen, sodass die anderen Beobachtungen Kombinationen davon sind.

Das übergeordnete Ziel dieser Vorgehensweise ist, dass die Auswahl der Routen sich nicht mehr nur ausschließlich auf die Geometrie des Gebäudeplans und der Routen bezieht, sondern zusätzlich weitere Eigenschaften der Routen einbezogen werden. Mit anderen Worten wird eine gegebene Menge von Routen basierend auf deren Ähnlichkeit zu extremen Beobachtungen (Archetypen) gruppiert.

Zusammengefasst werden in diesem Kapitel Ansätze präsentiert, mit denen geospatiale Trajektorien verglichen werden können. Das Ziel ist die Auswahl einer möglichst kleinen Menge möglichst unterschiedlicher Routen. Daher werden in diesem Kapitel folgende drei Herausforderungen untersucht.

1. Die erste Herausforderung liegt in der Erstellung einer Bewertungsfunktion zur Sortierung geospatialer Trajektorien. Diese soll sich ausschließlich auf den Gebäudeplan beziehen und ermöglichen, (alternative) Routen zu vergleichen und auszuwählen.

2. Anschließend wird ein Verfahren vorgestellt, mit dem eine Einschränkung der Alternativen auf extreme Exemplare ermöglicht wird. Hierzu wird die archetypische Analyse auf verschiedene Merkmale der Routen angewendet und somit werden archetypische Routen definiert.

3. Schließlich wird basierend auf den Erkenntnissen der archetypischen Routen ein neuartiges Distanzmaß namens archetypische Distanz definiert, das zum Vergleich der Unterschiedlichkeit von Routen verwendet werden kann.

\subsection{Clusteranalyse und Distanzmaße}

Wie eingangs erwähnt, werden in diesem Kapitel Verfahren vorgestellt, um aus einer großen Menge von Routen, die sich potentiell nur gering unterscheiden, eine kleine Menge stark unterschiedlicher Routen zu extrahieren. Der Fokus liegt also auf dem Vergleich von geospatialen Trajektorien, das heißt von zeitlich geordneten Folgen von Punkten im Raum.

Der erste hier vorgestellte Ansatz bewertet Routen mittels einer zuvor berechneten Überlastungswahrscheinlichkeit und erstellt somit ein Scoring (siehe Abschnitt 4.3). Der zweite Ansatz vergleicht Routen mittels der archetypischen 
Analyse (Abschnitt 4.4.1). Basierend darauf wird schließlich ein neuartiges Distanzmaß für geospatiale Trajektorien vorgestellt (Abschnitt 4.4.2).

Dieser Abschnitt erläutert die entsprechenden Grundlagen: es wird die grundlegende Vorgehensweise der Clusteranalyse vorgestellt (4.2.1), die archetypische Analyse (4.2.2) sowie bestehende Distanzmaße (4.2.3).

\subsubsection{Clustering-Algorithmen}

Die Clusteranalyse ist eine Disziplin des Data-Minings, bei dem es um die Gewinnung von Wissen aus bereits bestehenden Daten geht. Das grundsätzliche Ziel der Clusteranalyse ist die Einteilung von Beobachtungen in Klassen oder Gruppen (Cluster), wobei ähnliche Beobachtungen gleichen Gruppen zugeordnet werden und unähnliche Beobachtungen entsprechend in unterschiedliche Gruppen [116]. Hierdurch sollen Erkenntnisse bezüglich der Ähnlichkeiten und Unterschiede der Beobachtungen, aber auch der erstellten Gruppen, gewonnen werden.

Die Zuordnung von ähnlichen Beobachtungen in die gleiche Gruppe basiert auf Merkmalen der Beobachtungen. Für die Berechnung der Ähnlichkeit ist somit ein Ähnlichkeits- oder Distanzmaß notwendig, das auf die Merkmale der Beobachtungen angewendet wird. In der Literatur existieren zahlreiche Maße mit jeweiligen Vor- und Nachteilen (siehe beispielsweise [9] oder [67]), die jedoch allesamt basierend auf den Skalen der Merkmale gewählt werden. Es wird grundsätzlich zwischen drei Kategorien der Merkmalsskalierung unterschieden [97]:

1. metrisch: numerische, intervallskalierte Werte wie etwa die erreichte Punktzahl $s$ in einem Test mit $s \in[0,100]$.

2. nominal: unterscheidbare Werte ohne natürliche Rangfolge wie etwa Blutgruppen im AB0-System.

3. binär: Werte, die auf 0 oder 1 abgebildet werden können, wie etwa das Vorhandensein oder Fehlen eines bestimmten Kriteriums.

Bei der Zuordnung von Beobachtungen in Gruppen gibt es zwei unterschiedliche Vorgehensweisen: das „harte“ (crisp) und „weiche“ (fuzzy) Clustering. Bei der harten Gruppenzuordnung wird die Beobachtung genau einer ermittelten Gruppe zugeordnet. Sollte es bei den ermittelten Gruppen Überschneidungen geben, so wird die Beobachtung derjenigen Gruppe zugeordnet, bei der die Ähnlichkeit am höchsten ist. Bei der weichen Gruppenzuordnung wird jede Beobachtung zu einem bestimmten Grad jeder ermittelten Gruppe zugeordnet. Die Zugehörigkeit einer Beobachtung zu einer Gruppe liegt also in einem Wertebereich von 0 (keine Zuordnung) und 1 (perfekte Zuordnung) und summiert sich für eine Beobachtung über alle Gruppen auf 1. Die harte Gruppenzuordnung kann also als Spezialfall der weichen Zuordnung angesehen werden. 


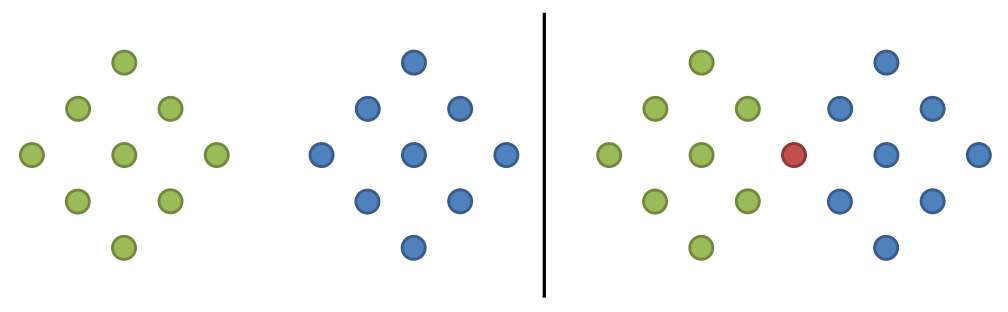

Abbildung 4.1: Fuzzy Clustering ist in der Lage die rot eingefärbte Beobachtung zu gleichen Teilen in beide Gruppen einzuordnen.

In Abbildung 4.1 ist ein stark vereinfachtes Beispiel zu sehen, bei dem mehrere Beobachtungen in einer zweidimensionalen Fläche dargestellt sind. Auf der linken Seite sind die Beobachtungen derart angeordnet, dass sie genau zwei Cluster bilden. Die harte Gruppenzuordnung würde demnach die linken Beobachtungen einer ,grünen“ Gruppe zuordnen und die rechten Beobachtungen einer „blauen“ Gruppe. Bei der weichen Gruppenzuordnung hätten die Beobachtungen ebenfalls allesamt eine hohe Gruppenzugehörigkeit zu den jeweiligen Clustern. Anders wäre dies bei dem Beispiel auf der rechten Seite, bei der die Beobachtungen näher zueinander stehen. Bei der harten Gruppenzugehörigkeit muss bei der rot eingefärbten Beobachtung entschieden werden, zu welcher Gruppe sie mehr gehört. Bei exakt gleicher Ähnlichkeit müsste zufällig entschieden werden. Mit der weichen Gruppenzuordnung hingegen kann man dieses Problem umgehen, indem die Gruppenzugehörigkeit der roten Beobachtung mit jeweils $50 \%$ ausgedrückt wird.

Bei den Algorithmen zur Clusteranalyse wird grundsätzlich zwischen den dichtebasierten, hierarchischen und partitionierenden Verfahren unterschieden, die im Folgenden kurz beschrieben werden [121].

Dichtebasierte Verfahren Grundsätzlich verfolgen dichtebasierte Verfahren das Ziel, Gruppen zu bilden, in denen eine hohe Dichte herrscht und die durch Bereiche geringerer Dichte voneinander separiert sind. Eine hohe Dichte liegt vor, wenn die Beobachtungen in Bezug auf ein gewähltes Distanzmaß nahe beieinander liegen. Die Zuordnung findet dann mittels eines Schwellwerts für die Dichte statt, der innerhalb eines Clusters nicht unterschritten werden darf. Beobachtungen, die keiner Gruppe mit hoher Dichte zugeordnet werden, werden als Rauschen oder Outlier bezeichnet. Beispiele für dichtebasierte Clusteringverfahren sind DBSCAN [66] oder dessen Erweiterung OPTICS [5].

Hierarchische Verfahren Das Ziel hierarchischer Clusteringverfahren ist das Gruppieren von Beobachtungen innerhalb einer Hierarchie, sodass diese $\mathrm{Zu}-$ sammenhänge als Baumdiagramm (auch Dendogramm genannt) dargestellt werden können. Je tiefer im Baum zwei Beobachtungen zusammengeführt werden können, desto spezifischer ist die Gruppenzugehörigkeit [184]. Die Erstel- 
lung der hierarchischen Cluster kann entweder bottom-up (auch agglomerativ genannt) oder top-down (divisiv) geschehen. Bei der agglomerativen Vorgehensweise wird initial jede Beobachtung als eigene Gruppe angesehen. Anschließend werden sukzessive ähnliche Cluster zusammengefügt, bis eine bestimmte Abbruchbedingung erfüllt ist. Die divisive Vorgehensweise startet mit einem großen Cluster, in dem alle Beobachtungen enthalten sind, und führt solange eine Aufteilung der Gruppen fort, bis ebenfalls ein Abbruchkriterium erfüllt ist. Die finalen Cluster ergeben sich bildlich gesprochen durch einen horizontalen Schnitt des Dendogramms, sodass je nach Höhe des Schnitts unterschiedlich viele und demnach unterschiedlich spezifische Gruppen zurück gegeben werden. Ein Beispiel für hierarchische Clusteringverfahren ist DIANA [121].

Partitionierende Verfahren Die Idee hinter der Verwendung partitionierender Verfahren ist die Annahme, dass in den Beobachtungen eine feste Anzahl an natürlichen Gruppen enthalten ist. Partitionierende Verfahren starten demnach mit einer vorgegebenen Anzahl an zufälligen Repräsentanten der Gruppen und versuchen solange die Zuordnung der Beobachtungen zu den Klassen zu verändern, bis entweder eine obere Schranke an Ausführungsschritten oder eine bestimmte Qualität der Gruppen erreicht ist beziehungsweise keine Umordnung der Beobachtungen mehr stattfindet. Ein Beispiel für ein partitionierendes Clusteringverfahren ist k-Means [137]. Hierbei wird der Repräsentant einer Gruppe durch das Gruppenzentrum bestimmt, was über den Mittelwert der Beobachtungen der Gruppe berechnet wird. Die archetypische Analyse, die im folgenden Abschnitt näher erläutert wird, gehört ebenfalls zu den partitionierenden Clusteringverfahren.

\subsubsection{Archetypische Analyse}

Die archetypische Analyse [36] ist eine Technik zur statistischen Datenanalyse, deren Ergebnisse vergleichbar sind mit den Resultaten von ClusteringMethoden wie etwa dem partitionierenden k-means-Clustering [100]. Das Ziel ist also das Separieren von Beobachtungen, die innerhalb eines Merkmalsraums verteilt sind, sodass sinnvolle Partitionen entstehen. Das k-meansClusteringverfahren extrahiert Schwerpunkte innerhalb der Beobachtungen (Cluster-Zentren) und weist die übrigen Datenpunkte diesen Cluster-Zentren zu.

Im Gegensatz zu traditionellen Clustering-Methoden sucht die archetypische Analyse nach Beobachtungen auf dem äußeren Rand des Datenraums und approximiert die konvexe Hülle der Daten. Mit anderen Worten versucht ein entsprechender Algorithmus Datenpunkte zu finden, die maximal weit voneinander entfernt sind. Diese Datenpunkte werden Archetypen genannt, können also auch als Extrema, Prototypen oder Originale umschrieben werden.

Abbildung 4.2 zeigt beispielhafte Ergebnisse, die mittels k-Means und arche- 


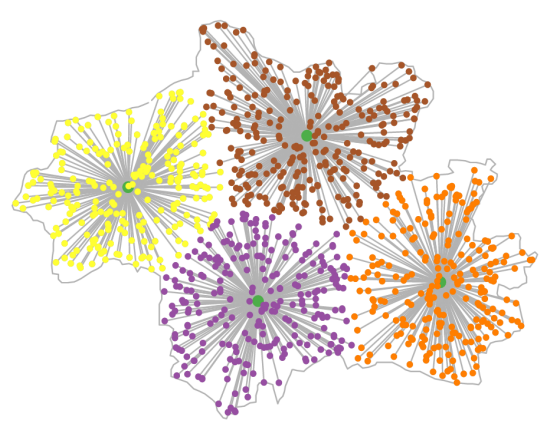

(a) k-Means

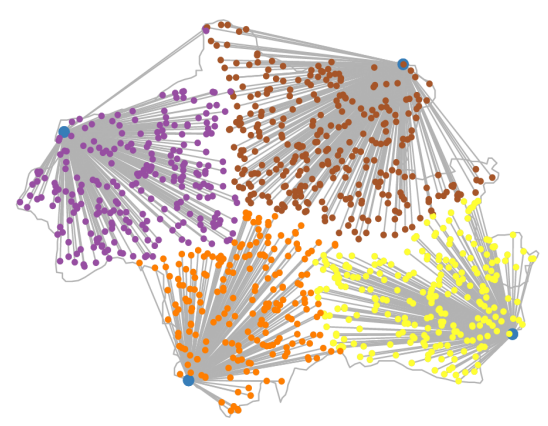

(b) Archetypische Analyse

Abbildung 4.2: Darstellung der unterschiedlichen Wahl der Repräsentanten mit der Zuordnung zu den jeweils ermittelten Gruppen. Abbildung aus [68]

typischer Analyse berechnet wurden. Während k-Means die Cluster-Zentren als Repräsentanten für die Gruppen wählt (siehe Abbildung 4.2a), wählt die archetypische Analyse Repräsentanten am Rand des Datenraums (Abbildung $4.2 \mathrm{~b})$.

Der grundlegende Algorithmus zur archetypischen Analyse ist ein iterativer Ansatz, der zwei Schritte abwechselnd wiederholt [36], [162]. Das Ziel ist dabei eine möglichst genaue Beschreibung der konvexen Hülle des Datenraums mittels vergleichsweise wenigen Datenpunkten - den Archetypen. Dies wird mittels der Lösung eines linearen Optimierungsproblems erreicht.

Gegeben ein Datensatz mit $N$ Beobachtungen, wobei im Kontext der vorliegenden Arbeit eine Beobachtung genau eine alternative Route darstellt. Jede Beobachtung besitzt $m$ Eigenschaften wie beispielsweise die absolute Länge der Route oder die Anzahl der Richtungsänderungen. Der Datensatz wird durch eine $N \times m$ Matrix $X$ repräsentiert. Als partitionierendes Clusteringverfahren berechnet die archetypische Analyse nun eine definierte Anzahl an $k$ Archetypen. Dazu versucht der Algorithmus eine $k \times m$-dimensionale Matrix $Z$ durch die Minimierung der Residuenquadratsumme $(R S S)$

$$
R S S=\left\|X-\alpha Z^{T}\right\|_{2}
$$

zu finden. $\|\cdot\|_{2}$ repräsentiert dabei eine passende Matrixnorm, im vorliegenden Falle die Spektralnorm, und $\alpha$ stellt somit die $N \times k$ Koeffizientenmatrix dar, die benötigt wird, um den Datensatz $X$ aus einer gegebenen Menge von Archetypen $Z$ zu generieren beziehungsweise anzunähern.

Im ersten der zwei Iterationsschritte versucht der Algorithmus Gleichung 4.1 unter Berücksichtigung der Bedingungen

$$
\alpha_{i j} \geq 0 \text { und } \sum_{j=1}^{k} \alpha_{i j}=1
$$




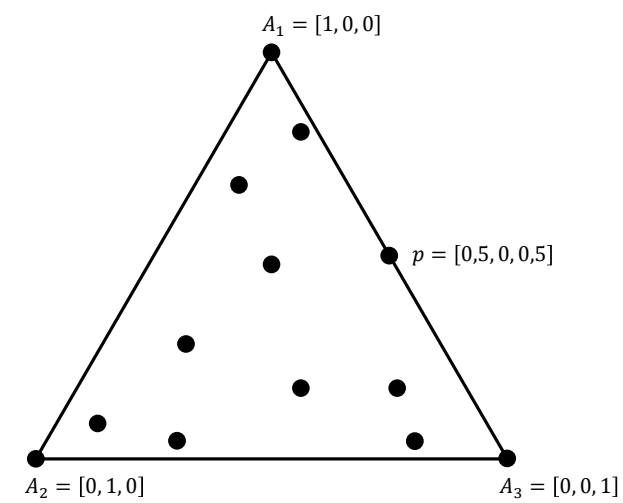

Abbildung 4.3: Zusammenhang zwischen der Koeffizientenmatrix $\alpha$ und der Eigenschaft der weichen Gruppierung (fuzzy Clustering).

zu minimieren, wobei $i=1, \ldots, N$. Matrix $Z$ beinhaltet dabei die identifizierten Archetypen und ist eine konvexe Kombination der tatsächlichen Datenpunkte. Das bedeutet, dass Matrix $Z$ durch

$$
Z=X^{T} \beta
$$

dargestellt werden kann, wobei $\beta$ eine $N \times k$ Matrix ist.

Im zweiten Iterationsschritt versucht der Algorithmus Gleichung 4.3 unter Berücksichtigung der Bedingungen

$$
\beta_{j i} \geq 0 \text { und } \sum_{i=1}^{N} \beta_{j i}=1
$$

abzuschätzen, wobei $j=1, \ldots, k$.

Der vorgestellte Ansatz kann somit auch als "Alternating Least Square“Algorithmus (Ansatz der alternierenden kleinsten Quadrate) bezeichnet werden, da er zwischen der Berechnung des besten Koeffizienten $\alpha$ für gegebene Archetypen $Z$ und der Berechnung der besten Archetypen $Z$ für einen gegebenen Koeffizienten $\beta$ iteriert.

Abbildung 4.3 soll den Zusammenhang zwischen der Koeffizientenmatrix $\alpha$ und der Eigenschaft der weichen Gruppierung (fuzzy Clustering) verdeutlichen. In diesem Beispiel hat die archetypische Analyse drei Archetypen $A_{1}$, $A_{2}$ und $A_{3}$ berechnet. Eine Beobachtung, die dem Archetypen $A_{1}$ entspricht, hätte in der Koeffizientenmatrix $\alpha$ entsprechend den Eintrag [1,0,0]. Sie wäre also im Sinne einer weichen Gruppenzuordnung ein „perfektes“ Mitglied der ersten Gruppe. Die Beobachtung $p$ im rechten Bereich der Abbildung liegt in der Darstellung genau in der Mitte zwischen den zwei Archetypen $A_{1}$ und $A_{3}$, weshalb eine weiche Zuordnung in beide Gruppen stattfinden würde. Der entsprechende Eintrag in der Koeffizientenmatrix $\alpha$ wäre demnach $[0,5,0,0,5]$.

Die Ausführung der archetypischen Analyse terminiert immer, jedoch nicht 


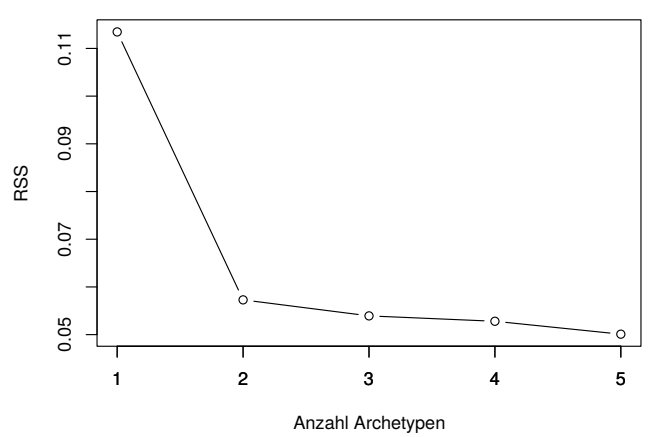

Abbildung 4.4: Beispielhaftes Eigenwertediagramm der resultierenden $R S S$ für unterschiedliche Werte von $k$. Das Abflachen der Kurve bei $k=2$ deutet auf eine passende Wahl hin.

notwendigerweise bei dem globalen Minimum der $R S S$. Das bedeutet, dass die gefundene Annäherung der konvexen Hülle des Datenraumes unter Verwendung von $k$ Punkten nicht zwingend die genaueste ist, sondern eben nur ein lokales Minimum. Darüber hinaus existiert keine universelle Regel für die initiale Bestimmung der Anzahl der Archetypen $k$. Ein für gewöhnlich verwendeter Ansatz ist der Scree-Test (auch „Ellbogenkriterium“ genannt), bei dem ein Abflachen des Eigenwertediagramms der $R S S$ (Scree-Plot der $R S S$ ) auf eine möglicherweise gute Wahl für $k$ hinweist.

Abbildung 4.4 verdeutlicht dies. Zu sehen ist ein beispielhaftes Eigenwertediagramm der resultierenden $R S S$ für unterschiedliche Werte für $k$. Während bei der Verwendung von $k=2$ statt $k=1$ eine starke Verbesserung der Annäherung der konvexen Hülle zu erkennen ist (starker Abfall der $R S S$ ), ist dies bei der Hinzunahme eines weiteren Archetypen $(k=3)$ nicht der Fall. Daher bietet sich in diesem Szenario die Verwendung von $k=2$ Archetypen an.

Weitere Details zur Vorgehensweise der archetypischen Analyse werden während der Erläuterungen zur Berechnung archetypischer alternativer Routen sowie in der Evaluation gegeben (Abschnitte 4.4.1 und 4.4.4). Ferner werden in der Literatur weitere Fragestellungen wie numerische Aspekte, Stabilität, Komplexität der Berechnung, Robustheit sowie konkrete Anwendungen diskutiert [36], [70], [71], [162].

\subsubsection{Distanzmaße}

Der Kern der vorliegenden Arbeit, nämlich die Berechnung und Verarbeitung von alternativen Routen in komplexen Umgebungen, ist eng verwandt mit der Messung der Ähnlichkeit von Routen: das Ziel ist es in beiden Fällen unterschiedliche Routen zu erkennen. Dafür können Ähnlichkeits- oder Distanzmaße genutzt werden, wie sie auch bei der oben beschriebenen Clusteranalyse verwendet werden. Außerdem lässt sich folgender Zusammenhang beobachten: Äquivalente Routen und durch Clustering ermittelte Gruppen haben jeweils 
eine niedrige Distanz zueinander, wohingegen alternative Routen und unterschiedliche Cluster jeweils eine hohe Distanz zueinander besitzen.

Wenn beispielsweise die Distanz zwischen Punkten in einem zwei- oder dreidimensionalen Raum berechnet werden muss, so ist eine angemessene Distanz die Luftlinie zwischen den Punkten. Wenn allerdings die Distanz von Routen gemessen werden soll, so wird dies schnell eine überraschend komplexe Fragestellung. Grundsätzliche Strategien zur Berechnung der Ähnlichkeit von Trajektorien (sortierte Folgen von Ortspunkten) können das Fokussieren auf entweder einzelne Punkte, auf höherdimensionale Objekte wie Liniensegmente, oder auf Mengen von Punkten sein. Es existieren zahlreiche Algorithmen, die für die Berechnung der Ähnlichkeit von Routen verwendet werden können, jeweils mit Stärken und Schwächen bezüglich gegebener Anforderungen, aber auch bezüglich Komplexität, Laufzeit sowie Empfindlichkeit gegenüber Ausreißern und der Abtastrate [178]. Im Folgenden werden die wichtigsten Kategorien solcher Algorithmen kurz vorgestellt.

Bei der ersten Kategorie von Algorithmen wird die Distanz zwischen den Trajektorien durch die Distanz der Punkte repräsentiert. So berechnet die Closest Pair Distance (CPD) [34] die Distanz zwischen allen Punkten der Trajektorien und gibt die minimale Distanz als Antwort zurück. Die Sum of Pair Distance (SPD) [3] berechnet die Distanz aller Punktpaare und kann somit nur bei Trajektorien mit derselben Länge angewendet werden. Diese Einschränkung kann umgangen werden, indem in der kürzeren Trajektorie Punkte mittels Interpolation eingefügt werden. Nachteilig bei der CPD ist die hohe Komplexität durch den Vergleich der Distanzen aller Punkte. Bei der SPD ist es die potentiell notwendige Interpolation. Beide Algorithmen sind zudem anfällig gegenüber Ausreißern.

Die zweite Kategorie von Algorithmen zur Messung der Ähnlichkeit von Trajektorien adaptiert bekannte Ansätze zur Berechnung der Distanz zwischen Zeichenfolgen. Die grundsätzliche Idee ist es, entweder gemeinsame Teiltrajektorien zu finden oder die eine Trajektorie in die andere zu übersetzen. Longest Common Subsequence (LCS) [176] ist eine Methode, die ursprünglich aus dem Bereich der Spracherkennung stammt und die Länge der längsten gemeinsamen Teilsequenz berechnet und zurückliefert. LCS ist robust gegenüber Rauschen und Ausreißern, da der Ansatz in der Lage ist, Punkte zu ignorieren. Bei der Edit Distance on Real Subsequences (EDR) [29], [133] wird die Anzahl der Operationen gezählt, die benötigt wird, die Vergleichstrajektorie in die Referenztrajektorie zu übersetzen. Als Operationen stehen Einfügen, Löschen und Ersetzen von Ortspunkten zur Verfügung. Eine weitere sehr verbreitete Methode ist Dynamic Time Warping (DTW) [187], bei der die notwendigen Kosten für eine optimale Angleichung von zwei Trajektorien als deren Distanz angesehen werden.

Schließlich existiert eine Kategorie von Algorithmen, die den zeitlichen Aspekt der Trajektorien außer Acht lässt und sich ausschließlich auf die Geometrie bezieht. So wird die Hausdorff Distance (HD) [102] ursprünglich für 
die Berechnung der Ähnlichkeit von Punktmengen verwendet. Sie berechnet das Maximum der minimalen Distanzen zwischen den zwei Trajektorien und beachtet deshalb ausschließlich die räumliche Entfernung der Punkte ohne die zeitliche Dimension. Eine bekannte Erweiterung ist durch die Fréchet Distance (FD) [81] gegeben. Sie wird oft durch das Bild eines angeleinten Hundes und dessen Besitzers beschrieben: die FD ist die minimale Länge einer Leine, die den Hund mit dem Besitzer verbindet, während diese auf der jeweiligen Trajektorie entweder vorwärts laufen oder stehen bleiben. Die Lösung dieses Optimierungsproblems ist sehr berechnungsintensiv. Eine effizientere Abwandlung ist die diskrete Fréchet-Distanz [185], bei der die Frage nach der kürzesten Leine nur auf den Punkten der Route gestellt wird und nicht wie bei der generellen FD auf parametrisierten Kurven.

Alle Verfahren zur Berechnung der Distanz zwischen Trajektorien teilen die Eigenschaft, dass sie nicht den zugrundeliegenden geografischen Raum, also beispielsweise einen Gebäudeplan, berücksichtigen. Es existieren vereinzelte Erweiterungen wie etwa die Homotopic Fréchet Distance [27], die jedoch eine immense Laufzeit besitzen, und deshalb nicht zur Analyse von großen Mengen von Routen verwendet werden können.

\section{3 Überlastungswahrscheinlichkeit zur Bewertung von Routen}

Der im Folgenden beschriebene Ansatz zum Vergleich geospatialer Trajektorien bezieht sich auf die erste Herausforderung dieses Kapitels (Abschnitt 4.1). Es wird eine kartenbasierte Bewertungsfunktion vorgestellt, die gegebenen Routen Realwerte (Scores) zuordnet. Die Ergebnisse dieser Bewertungsfunktion können dazu verwendet werden, eine Sortierung für eine Menge von (alternativen) Routen zu erstellen, um diese anschließend zu klassifizieren und auszuwählen. Allgemein gesprochen ermöglicht es dieser Ansatz, die Ähnlichkeit von Routen zu beschreiben.

Das sogenannte Scoring, das hier Anwendung findet, ist grundsätzlich eine Methode zur Entscheidungsfindung. Dazu werden Scores eingeführt, um schwer abwägbare Sachverhalte wie etwa qualitative oder subjektive Fakten vergleichbar zu machen. In einem weiteren Sinne wird Scoring als ein analytischer oder statistischer Ansatz verwendet, der durch die Verwendung von wenigen gesammelten Daten und empirischen Werten eine Risikoeinschätzung ermöglicht. Nach dem Scoring der Fakten, also der Bewertung derselben, werden diese sortierbar und es kann entsprechend eine Rangfolge erstellt werden.

Grundsätzlich bewertet der im Folgenden vorgestellte Algorithmus die Ähnlichkeit von alternativen Routen basierend auf Informationen, die implizit aus gewöhnlichen Gebäudeplänen generiert wurden. In Abschnitt 4.3.1 werden die hierfür notwendigen Eingaben und Rahmenbedingungen definiert und es wird die zu erstellende Bewertungsfunktion formalisiert. Anschließend wird in Ab- 


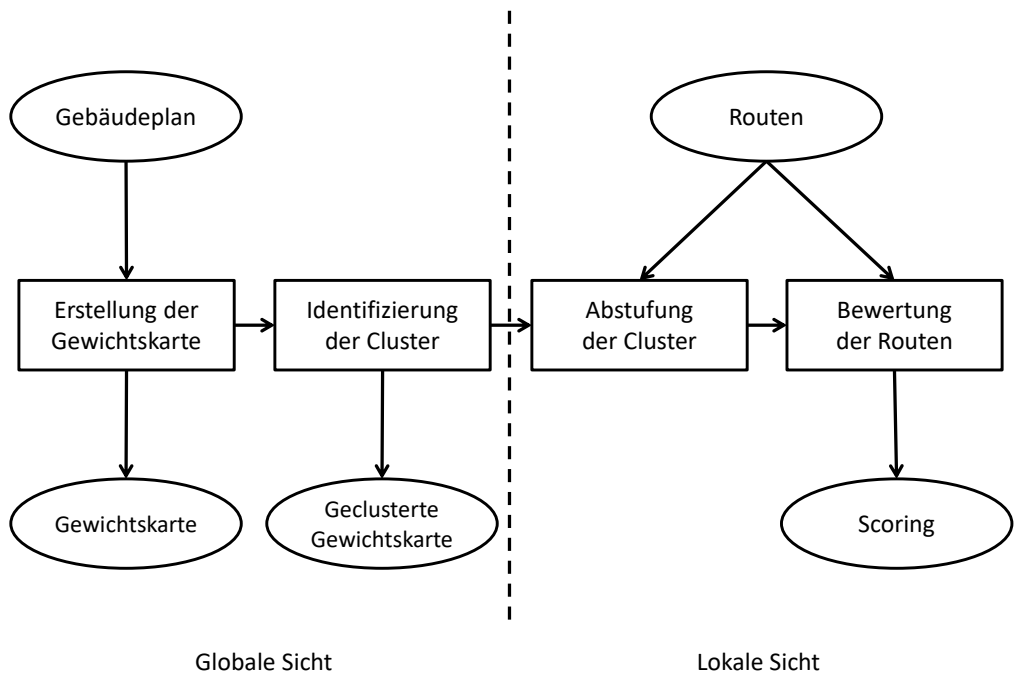

Abbildung 4.5: Grundlegende Vorgehensweise des Ansatzes zur Bewertung von Routen mittels Überlastungswahrscheinlichkeit.

schnitt 4.3.2 der Begriff der Überlastungswahrscheinlichkeit diskutiert sowie die Herleitung der dazugehörigen Idee illustriert. Außerdem wird die gewählte Bewertungsfunktion begründet und deren globale und lokale Sichtweise bei der Bewertung von alternativen Routen eingeführt.

Der eigentliche Algorithmus zur Bewertung besteht wiederum aus mehreren Schritten, die nicht notwendigerweise aufeinander bauen, sondern durchaus unabhängig voneinander verwendet werden können. In Abschnitt 4.3.3.1 wird die von der Bewertungsfunktion verwendete Gewichtskarte beschrieben, während in Abschnitt 4.3.3.2 eine Definition für potentiell verkehrsreiche Areale und weitere Abstufungen davon im Sinne der Überlastungswahrscheinlichkeit gegeben wird. Es folgt die eigentliche Bewertung der alternativen Routen in Abschnitt 4.3.3.3.

Schließlich wird in Abschnitt 4.3.4 der vorgestellte Ansatz basierend auf unterschiedlichen Gebäudeplänen evaluiert und diskutiert.

Abbildung 4.5 illustriert nochmals den grundlegenden Ablauf: Die Eingabe ist ein Gebäudeplan, der für die Erstellung einer Gewichtskarte verwendet wird, in welcher anschließend Cluster berechnet werden. Diese Schritte besitzen eine globale Sicht. Anschließend werden Routen als zusätzliche Eingabe dazu verwendet, die identifizierten Cluster weiter abzustufen, und die Routen werden schließlich selbst bewertet. Dies resultiert in einem Scoring der Routen. Da die letzten Schritte bezogen auf eine konkrete Menge von Routen arbeiten, besitzen diese eine lokale Sicht. 


\subsubsection{Definitionen und Ziel}

Die Eingabe des Algorithmus ist eine unsortierte Liste $R=\left\{r_{1}, \ldots, r_{n}\right\}$ mit $n$ Trajektorien $r=\left(x_{1}, y_{1} ; \ldots ; x_{m}, y_{m}\right) \in R$ in der Fläche. Die Trajektorien können unterschiedlich lang sein, haben jedoch denselben Startpunkt $s$ und denselben Endpunkt $t$. Zusätzlich ist jede Trajektorie $r_{i}$ genau einer Äquivalenzklasse $c_{i}=$ $\left[r_{i}\right]$ zugeordnet, die mittels der Homotopie-Relation definiert wurden (siehe Abschnitt 3.2).

Neben der Liste an Trajektorien ist ein Gebäudeplan $M \subset \mathbb{B}^{m \times n}$ in der Darstellung eines Belegungsrasters gegeben. In dieser Darstellung werden begehbare Flächen durch weiße Pixel repräsentiert und Hindernisse wie etwa Wände oder Möbel durch schwarze Pixel. Eingezeichneten Türsymbole werden im Vorfeld entfernt, um eine durchgehende Erreichbarkeit zu erhalten.

Das Ziel des Algorithmus ist die Erstellung einer Bewertungsfunktion

$$
s_{M}:\left(\mathbb{Z}^{2}\right)^{n} \rightarrow \mathbb{R},
$$

die eine konstante Karte $M$ berücksichtigt und eine 2D-Trajektorie von beliebiger Länge $n$ in einen Realwert transformiert. Diese Bewertungsfunktion kann anschließend von einer Rangordnungsfunktion

$$
r_{M}:\left(\left(\mathbb{Z}^{2}\right)^{n_{i}}\right)^{l} \rightarrow \mathbb{R}^{l}
$$

verwendet werden, um $l$ (alternative) Routen zu bewerten und anschließend eine Rangordnung zu erstellen. Wie bereits in Abschnitt 4.3 angedeutet wurde und im folgenden Abschnitt 4.3.2 weiter diskutiert wird, bewertet der hier vorgeschlagene Algorithmus eine Liste an Routen bezüglich der Überlastungswahrscheinlichkeit. Routen, die einen hohen Score erhalten, werden als potentiell verkehrsreich eingeschätzt, während Routen mit einem niedrigen Score als potentiell verkehrsarm interpretiert werden.

\subsection{2 Überlastungswahrscheinlichkeit}

In diesem Abschnitt wird die Idee hinter dem Begriff der Überlastungswahrscheinlichkeit hergeleitet und die gewählte Bewertungsfunktion begründet. Die Idee zu potentiell verkehrsarmen und potentiell verkehrsreichen Flächen kam auf, als die durch den Penalty-Algorithmus (siehe Abschnitt 3.3.2) erstellten Gewichtskarten näher untersucht wurden. Abbildung 4.6 zeigt die Gewichtskarte für das Szenario „White House“, bei dem mithilfe des Penalty-Algorithmus eine Menge alternativer Routen, die von einem Raum oben links im Bild zu einem Raum unten rechts im Bild verlaufen, berechnet wurden. Wenn nun die Abbildung betrachtet wird, so ist ersichtlich, dass eine Stelle im Gebäudeplan existiert, die von allen implizit eingezeichneten Routen passiert werden muss (Türdurchgang bei Längengrad B). Es ist visuell offensichtlich, dass es an diesem Längengrad nur eine „heiße“ Fläche gibt, wohingegen es im linken Bereich der Karte einen Längengrad gibt, auf dem fünf „heiße“ Stellen angeordnet sind 


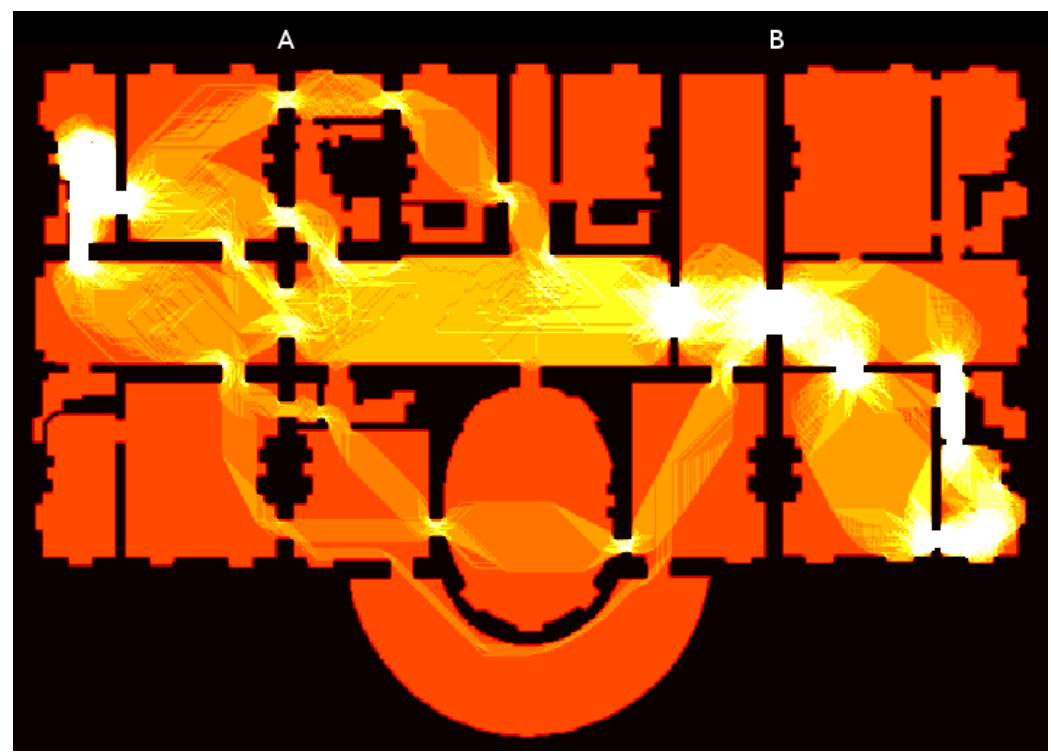

Abbildung 4.6: Gewichtskarte, die bei der Berechnung von alternativen Routen mithilfe des Penalty-Algorithmus erstellt wurde. Start der Routen ist der Raum links oben und Ziel der Raum rechts unten. Das Bild ist adaptiert von [179], Abbildung 9b.

(Längengrad A). Das bedeutet beispielsweise, dass es mehrere Alternativen gibt, falls ein Türdurchgang blockiert ist. Aus diesem Grunde wird der Gedanke der Überlastungswahrscheinlichkeit als eine Abschätzung definiert, mit der konkrete Flächen eines Gebäudeplans bewertet werden. Die Kernidee ist die Annahme, dass Punkte auf einer Karte, die häufig Teil eines kürzesten Pfades sind, tatsächlich auch häufig durchlaufen werden.

In der Literatur rechtfertigen Chen et al. in [30] die Notwendigkeit für stark unterschiedliche Routen in Straßennetzen mit der Tatsache, dass mehrere alternative Routen zeitgleich wegfallen können, falls ein gemeinsam verwendeter Pfad nicht mehr genutzt werden kann. Der in [30] vorgestellte Algorithmus zielt auf die Erstellung eines Alternativgraphen ab, der die Möglichkeit des gemeinsamen Scheiterns alternativer Routen reduziert, und umfasst zwei Schritte: Im ersten Schritt werden mittels einer Heuristik angemessene Kandidaten für alternative Routen konstruiert. Anschließend wird im zweiten Schritt überprüft, ob die Kandidaten gewisse Bedingungen erfüllen. Chen et al. spezifizieren Verbindungen, die möglicherweise ausfallen, mithilfe der Begriffe Verbindungszuverlässigkeit (link reliability) und Hochrisikoverbindung (high-risk link). Die Verbindungszuverlässigkeit ist dabei die Wahrscheinlichkeit einer ungewöhnlichen Verzögerung bei der Nutzung der untersuchten Verbindung, was beispielsweise mittels historischer Daten oder durch Verkehrssimulation abgeschätzt werden kann. Demnach ist eine Hochrisikoverbindung gegeben, wenn die Zuverlässigkeit der Verbindung unterhalb eines definierten Schwellwertes liegt. Zusammengenommen schlagen Chen et al. vor, dass alternative Routen 
das gemeinsame Durchlaufen von Hochrisikoverbindungen vermeiden sollten.

Auch wenn die Argumentation von Chen et al. konform zur Motivation der $\mathrm{zu}$ erstellenden Bewertungsfunktion ist, liegt der Fokus des hier beschriebenen Algorithmus auf alternativen Routen in Navigationsszenarien innerhalb von Gebäuden. Aus diesem Grund sind die Begriffe Verbindungszuverlässigkeit und Hochrisikoverbindung nicht vollständig geeignet. Stattdessen werden an dieser Stelle die Begriffe Überlastungswahrscheinlichkeit sowie potentiell verkehrsarme/verkehrsreiche Routen vorgeschlagen, die sich besser auf Flächen anstatt auf Verbindungen anwenden lassen. Ähnlich wie die Arbeit von Chen et al. besteht der hier vorzustellende Algorithmus auch aus zwei Schritten. Innerhalb eines Vorverarbeitungsschrittes wird eine Menge von Kandidaten für alternative Routen berechnet. Dies kann beispielsweise mittels des OnePatching-Algorithmus oder Penalty-Algorithmus (siehe Abschnitt 3.3) geschehen. Immer noch im Vorverarbeitungsschritt werden die Kandidaten überprüft, ob sie gewissen Anforderungen genügen, also als tatsächliche Alternativen genannt werden können (siehe Abschnitt 3.2). Im zweiten Schritt fokussiert sich der Algorithmus dann auf das Sortieren der Menge der gefundenen alternativen Routen basierend auf der Überlastungswahrscheinlichkeit, was am Ende eine kartenbasierte Rangordnung der alternativen Routen liefert. Im Gegensatz zu Chen et al. wird dazu jedoch kein Alternativgraph erstellt, sondern jede Route einzeln evaluiert.

Im weiteren Verlauf der Beschreibung des Algorithmus werden zwei unterschiedliche Sichtweisen eingenommen: eine lokale und eine globale Sicht. Eine lokale oder fallbezogene Sichtweise ist gegeben, wenn der Routing-Modus „eins$z u$-eins" verwendet wird. Das bedeutet, dass (alternative) Routen mit einem konkreten Startpunkt und einem konkreten Ziel evaluiert werden. Dies ist beispielsweise in Abbildung 4.6 der Fall. Der Routing-Modus „eins-zu-viele“ (ein Start, mehrere Ziele) verwendet ebenfalls eine lokale (fallbezogene) Perspektive. Ein konkreter Anwendungsfall dafür wäre zum Beispiel ein Flughafen, der einem Besucher Navigationshinweise von dessen aktuellem Standort zu mehreren Sehenswürdigkeiten präsentiert [159]. Schließlich existiert noch der Routing-Modus „viele-zu-viele“ (mehrere Starts, mehrere Ziele). Dabei wird eine globale Sicht angenommen, da nicht mehr konkrete (alternative) Routen behandelt werden, sondern Bezug auf den Gebäudeplan im Ganzen genommen wird.

Der in diesen Abschnitten vorgestellte Algorithmus verwendet zunächst auch eine solche globale Sicht. Dies ist der Fall bei der Erstellung einer Matrix $H$ (Abschnitt 4.3.3.1), die Teil der Bewertungsfunktion ist. Auch die Identifizierung von Clustern innerhalb der Matrix $H$ besitzt eine globale Sicht (Abschnitt 4.3.3.2). Erst ab der Erstellung der Funktion $g$ zur weiteren Abstufung der Cluster (ebenfalls Abschnitt 4.3.3.2), die ebenfalls Teil der Bewertungsfunktion ist, ist die Sicht des Algorithmus lokal (fallbezogen). Dies ist dadurch begründet, dass nun konkrete alternative Routen von einem gegebenen Startpunkt zu einem gegebenen Ziel zur Bewertung hinzugezogen werden. Auch die 
eigentliche Bewertung der alternativen Routen besitzt eine lokale (fallbezogene) Sicht (Abschnitt 4.3.3.3).

\subsubsection{Methodik}

Der in den folgenden Abschnitten vorgestellte Algorithmus besteht aus mehreren Schritten, die nicht notwendigerweise aufeinander aufbauen. Diese beinhalten die Erstellung einer Gewichtskarte, die Identifizierung potentiell verkehrsreicher Areale sowie die eigentliche Bewertung der alternativen Routen.

\subsubsection{Erstellung der Gewichtskarte}

Der erste Schritt des Algorithmus zur Bewertung alternativer Routen ist die Erstellung einer Matrix $H$ basierend auf einem gewöhnlichen Gebäudeplan, der als Eingabe zur Verfügung steht. Der Gebäudeplan $M \subset \mathbb{B}^{m \times n}$ wird durch eine einfache Bitmap repräsentiert, bei der weiße Pixel frei begehbare Flächen darstellen und schwarze Pixel Hindernisse wie Wände oder Möbel. Die $m \times$ $n$-Matrix $H$ wird derart initialisiert, dass an den Koordinaten, an denen $M$ weiße Pixel besitzt, eine 1 eingetragen wird und entsprechend eine 0 an den übrigen Stellen. Darüber hinaus wird ein gerichteter und gewichteter Graph $G=(V, E)$ erstellt, der für jeden weißen Pixel einen Knoten $v \in V$ enthält. Für zwei benachbarte, weiße Pixel wird eine Kante $e \in E$ eingefügt, wobei das Kantengewicht für horizontal und vertikal benachbarte Pixel mit $c(e)=1$ initialisiert wird und für diagonal benachbarte Pixel mit $c(e)=\sqrt{2}$.

Nun wählt der Algorithmus zufällig zwei Punkte $u, w$ aus, die begehbar in $M$ sind und die als Start- und Endpunkte für eine Kürzeste-Pfad-Suche in $G$ verwendet werden. Der hierbei entstehende kürzeste Pfad $r$ wird nun dazu verwendet, die Werte in der Matrix $H$ zu erhöhen. Gemäß der Erhöhung der Werte in Matrix $H$ werden auch die entsprechenden Gewichte der Kanten von $r$ in $G$ erhöht. Diese Vorgehensweise wird für eine zuvor definierte Anzahl an Iterationen $i$ wiederholt. Die Erhöhung der Werte und der Kantengewichte erfolgt im weiteren Verlauf durch eine einfache Verdoppelung.

Nach der Berechnung der Matrix $H$, die einer Gewichtskarte entspricht und als Heatmap visualisiert werden kann, müssen die Werte zur besseren Darstellung weiter verarbeitet werden. Um ein möglichst kontrastreiches Bild zu erhalten, wird Matrix $H$ an einem Schwellwert $z$ trunkiert. Das heißt, Werte, die größer als $z$ sind, werden auf $z$ gesetzt, alle anderen Werte werden beibehalten. Nach dem Begrenzen der Werte der Matrix $H$ werden diese normalisiert, was in $H_{i, j} \in[0,1]$ resultiert. In Abbildung 4.7 ist eine beispielhafte Ausgabe zu sehen, bei der nicht-begehbare Fläche wie etwa Wände zum Zwecke der Visualisierung grau eingefärbt ist. Elemente der Matrix $H$, die einen Wert von 0 besitzen, werden an korrespondierender Stelle der Karte $M$ durch die Farbe schwarz dargestellt und sind als potentiell verkehrsarme Flächen anzusehen. Entsprechend werden Elemente der Matrix $H$ mit einem Wert von 1 in der 


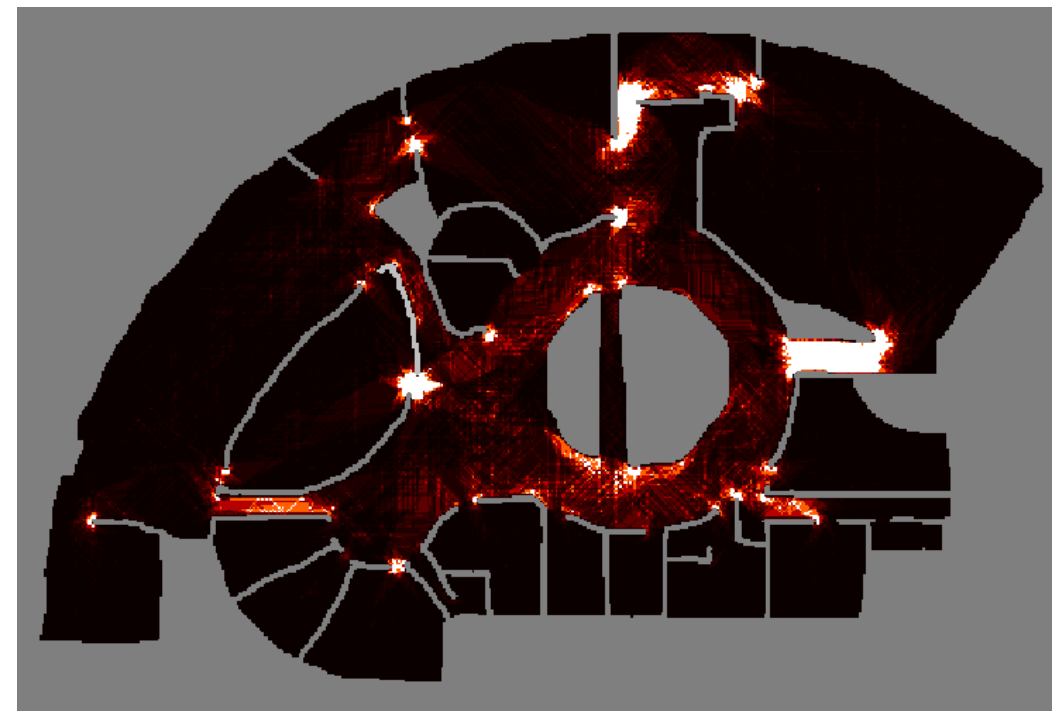

Abbildung 4.7: Beispiel einer Matrix $H$. Je heller (oder „heißer“) ein Pixel eingefärbt ist, desto höher ist die entsprechende Überlastungswahrscheinlichkeit. Nicht begehbare Flächen sind zum Zwecke der Visualisierung grau dargestellt.

Karte $m$ mit der Farbe weiß dargestellt und gelten als potentiell verkehrsreiche Stellen. Allgemein ausgedrückt bedeutet dies: je heller (oder im Sinne einer Heatmap „heißer") ein Pixel eingefärbt ist, desto höher ist die entsprechende Überlastungswahrscheinlichkeit.

\subsubsection{Identifizierung und Abstufung der Cluster}

Nach der pixel-basierten Identifizierung der Überlastungswahrscheinlichkeiten in einer Karte werden nun die potentiell verkehrsreichen Pixel in zusammenhängenden Flächen gruppiert. Dafür werden die Zeilen- und Spaltenindizes der Elemente der Matrix $H$ gesammelt, die den Maximalwert von 1 beinhalten, und in einer Menge $S$ gespeichert. Die Menge $S$ wird anschließend als Eingabe für den dichtebasierten Clustering-Algorithmus DBSCAN [66] mit minPts $=5$ verwendet, sodass mehr als 5 Pixel benötigt werden, um einen Cluster zu bilden (genauer: ein Objekt gilt als Kernobjekt, wenn mindestens minPts Objekte Dichte-erreichbar sind). Der Wert für den Parameter minPts wurde empirisch ermittelt und hat sich bei allen getesteten Gebäudeplänen mit einer angemessenen Auflösung bewährt. Der Parameter $\epsilon$ zur Bestimmung der Nachbarschaftslänge eines Objekts wird mittels der in [39] vorgestellten Heuristik automatisch gesetzt. Der Algorithmus gibt schließlich eine Menge von Clustern $C$ mit $c=\left(x_{1}, y_{1} ; \ldots ; x_{n}, y_{n}\right) \in C$ und $c \subseteq S$ zurück.

Zusammengefasst liefert der Schritt der Cluster-Identifizierung eine Menge von zusammenhängenden Flächen innerhalb der gegebenen Karte, bei denen eine hohe Überlastungswahrscheinlichkeit vorherrscht. Abbildung 4.8 zeigt ein beispielhaftes Ergebnis, bei dem die identifizierten Cluster grau eingefärbt sind. 


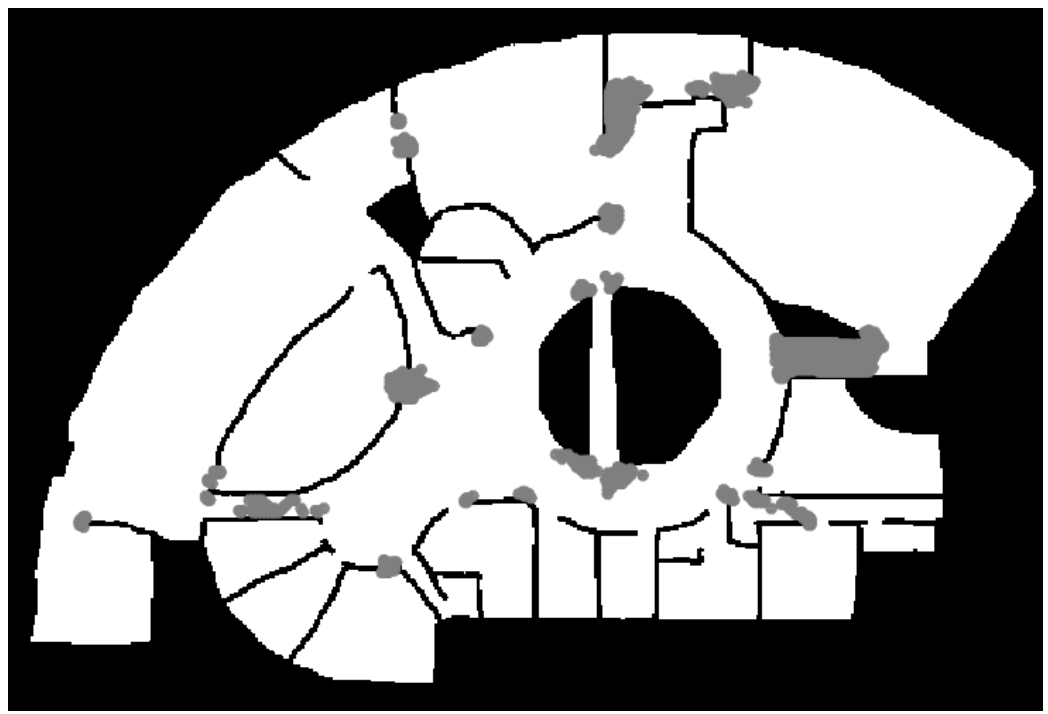

Abbildung 4.8: Identifizierte Cluster, bei denen eine hohe Überlastungswahrscheinlichkeit vorherrscht (grau eingefärbt).

Während der Erstellung der Gewichtskarte und der Identifizierung der Cluster hat der Algorithmus eine globale Perspektive. Nun werden die identifizierten, potentiell verkehrsreichen Flächen mittels konkreter (alternativer) Routen weiter verfeinert, was diesem Schritt eine lokale (fallbezogene) Sicht verschafft. Dazu wird eine Funktion $g: C \rightarrow[0,1]$ benötigt, die einen gegebenen Cluster $c \in C$ bezüglich der darin vorherrschenden Überlastungswahrscheinlichkeit bewertet. Somit werden die eingangs unbewerteten Flächen vergleichbar gemacht. Der in der Vorveröffentlichung [73] vorgestellte Ansatz verwendet dazu die relative Häufigkeit der Routen, die durch den zu bewertenden Cluster laufen. Somit ergibt sich:

$$
g(c)=\frac{\mid\{r \in R, \text { mit } r \cap c \neq \emptyset\} \mid}{|R|} .
$$

Funktion $g$ gibt einen Wert von 1 zurück, wenn jede der zur Verfügung gestellten alternativen Routen den entsprechenden Cluster durchlaufen, was offensichtlich als eine potentiell verkehrsreiche Fläche angesehen werden kann. Im Gegensatz dazu gibt Funktion $g$ den Wert 0 zurück, wenn keine einzige der zur Verfügung gestellten alternativen Routen die Fläche durchläuft, was als ein verkehrsarmes Areal interpretiert werden kann. Abbildung 4.9 zeigt eine beispielhafte Ausgabe nach der weiteren Verfeinerung der Cluster aus Abbildung 4.8. Die hierfür verwendeten alternativen Routen sind zum Zwecke der besseren Lesbarkeit der Grafik nicht eingezeichnet, jedoch die Start- und Zielpunkte der Routen als blaue Quadrate. Die Sättigung der Farbe der Cluster weist auf die Höhe der Bewertung hin, ausgehend von Weiß $(g(c)=0)$ bis hin zu Rot $(g(c)=1)$. Die begehbare Fläche ist zum Zwecke der besseren Lesbarkeit in grau dargestellt. Die Darstellung der eingefärbten Cluster vermittelt einen 


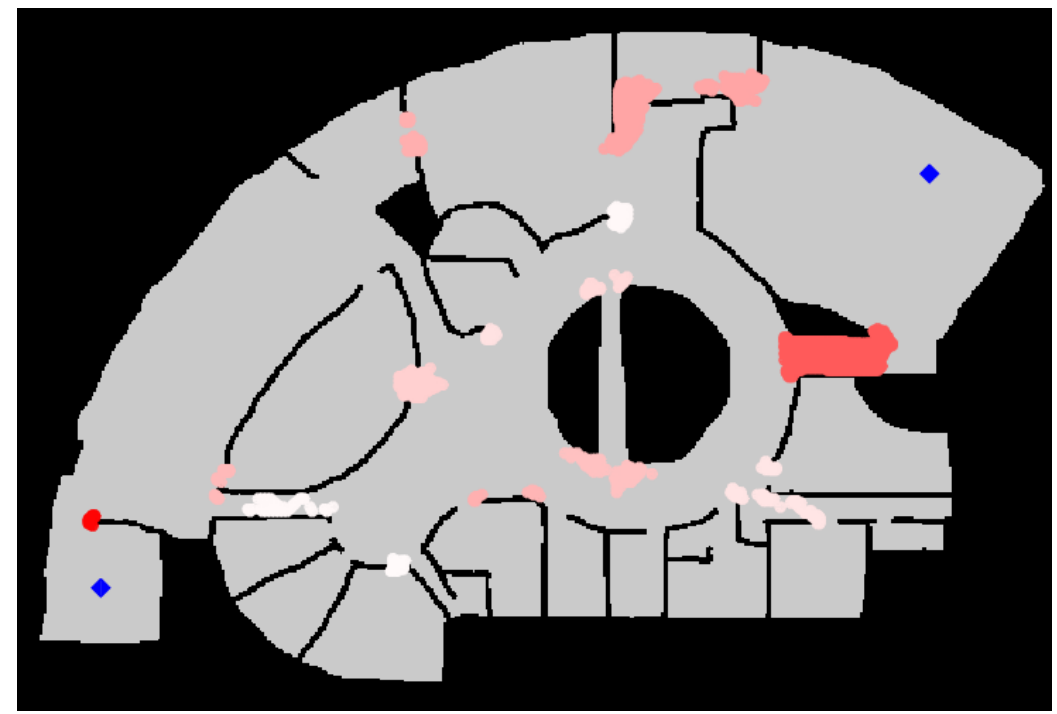

Abbildung 4.9: Cluster mit hoher Überlastungswahrscheinlichkeit, die mittels einer konkreten Menge alternativer Routen verfeinert wurden. Zur besseren Lesbarkeit der Grafik sind die alternativen Routen nicht eingezeichnet und die begehbare Fläche ist grau eingefärbt. Die blauen Quadrate markieren die Start- und Zielpunkte der alternativen Routen.

Eindruck in die Struktur eines Gebäudeplans, die einfacher zu lesen ist als die Gewichtskarte aus Abbildung 4.7. Es sei jedoch nochmals erwähnt, dass die Gewichtskarte aus einer globalen Perspektive heraus entstanden ist, während die eingefärbten Cluster eine lokale (fallbezogene) Sicht aufweisen.

\subsubsection{Bewertung der Routen}

Die eigentliche Bewertung einer alternativen Route $r \in R$ findet durch eine Funktion $s$ statt:

$$
s(r)=s_{1}(r)+s_{2}(r) \in[0,1] .
$$

Für den ersten Summanden $s_{1}(r)$ wird die in Abschnitt 4.3.3.1 beschriebene Gewichtskarte $H$ verwendet, wobei die einzelnen Bewertungen der Punkte der Route summiert werden und anschließend durch die Länge der Route dividiert wird. Somit gilt

$$
s_{1}(r)=\frac{1}{|r|} \sum_{(x, y) \in r} H_{x, y} .
$$

Der zweite Summand $s_{2}(r)$ bezieht sich auf die in Abschnitt 4.3.3.2 beschriebene Clustermenge $C$ und deren Bewertungsfunktion $g$. Für jeden Cluster $c \in C$ wird die Bewertung des Clusters mit dem relativen Anteil einer Route, der durch den Cluster verläuft, multipliziert. Somit gilt 


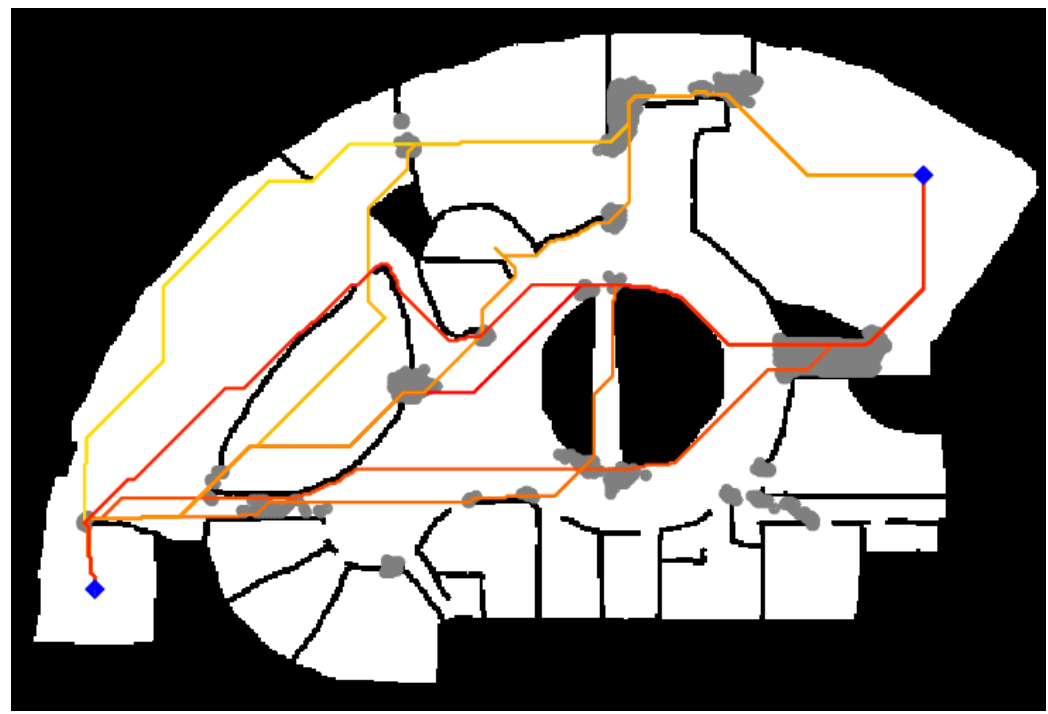

Abbildung 4.10: Beispielhafte Bewertung von Routen. Die berechnete Überlastungswahrscheinlichkeit der eingezeichneten alternativen Routen ist farbig visualisiert von niedrig (gelb) bis hoch (rot).

$$
s_{2}(r)=\sum_{c \in C} \frac{|c \cap r|}{|r|} g(c) .
$$

Die Summe in Gleichung 4.8 besteht also aus einem Teil, der eine globale Sicht verwendet (die Gewichtskarte) und einem Teil, der lokale (fallbezogene) Informationen verwendet (die durch Routen bewertete Cluster). Die Kombination der Summanden $s_{1}(r)$ und $s_{2}(r)$ bedeutet demnach, dass die Bewertung aller Punkte einer Route berücksichtigt wird und zusätzlich die Bewertung für Teile einer Route, an denen sie Cluster durchläuft. Schließlich wird die Summe normalisiert, um die Bewertungsfunktion $s(r)=s_{1}(r)+s_{2}(r) \in[0,1] \mathrm{zu}$ erhalten. Abbildung 4.10 zeigt ein beispielhaftes Ergebnis, bei der die berechnete Überlastungswahrscheinlichkeit der eingezeichneten alternativen Routen farbig dargestellt ist (von niedrig (gelb) bis hoch (rot)).

\subsubsection{Evaluation und Diskussion}

Um das Verhalten des Algorithmus zu illustrieren, werden in diesem Abschnitt die Bewertungsergebnisse von alternativen Routen in zwei unterschiedlichen Gebäudeplänen dargestellt und diskutiert. Die Bewertung, die einer konkreten Route zugeordnet wird, bezieht die Bewertungen aller Routen, die dieselbe Äquivalenzklasse teilen, mit ein. Das bedeutet, dass für die Bewertung einer Route der durchschnittliche Wert der Routen der Homotopieklasse verwendet wird. Damit werden kleinere Umwege einer Route innerhalb der Homotopieklasse nivelliert. Zum Zwecke der einfacheren Erklärung und Darstellung wurde dieses Detail in den vorherigen Definitionen nicht erwähnt und wird auch in 


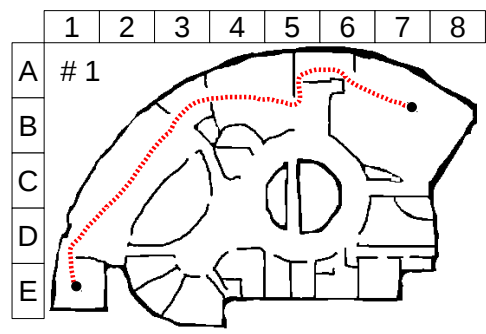

(a) $s\left(r_{1}\right)=0,62$

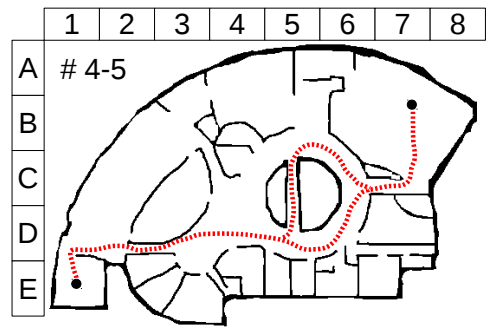

(c) $s\left(r_{4}\right)=0,87, s\left(r_{5}\right)=0,92$

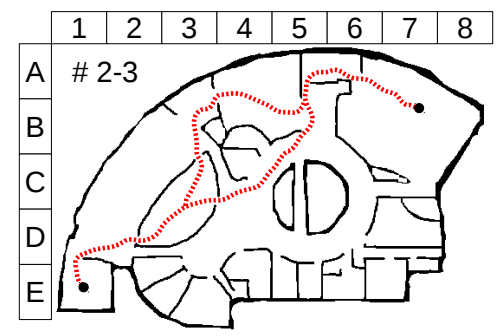

(b) $s\left(r_{2}\right)=0,65, s\left(r_{3}\right)=0,75$

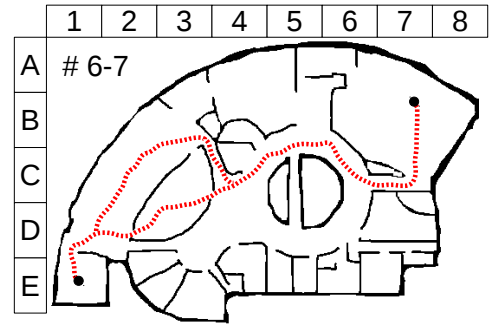

(d) $s\left(r_{6}\right)=0,93, s\left(r_{7}\right)=1$

Abbildung 4.11: Bewertete alternative Routen für das Szenario „Spa“.

den folgenden Beschreibungen ignoriert. Die Routen werden gemäß ihrer Bewertung aufsteigend sortiert, was bedeutet, dass der „erste Platz" die Route mit der geringsten Überlastungswahrscheinlichkeit ist.

\subsubsection{Szenario „Spa“}

Das erste Szenario namens „Spa“ ist eine vereinfachte Version eines realen Gebäudeplans, der bereits in Abschnitt 4.3 zur Erklärung des Algorithmus herangezogen wurde. Die bewerteten Routen sind in Abbildung $4.11 \mathrm{zu}$ sehen. Die Eingabe für den Algorithmus ist eine Menge von alternativen Routen in insgesamt sieben Homotopieklassen, die vom kleinen Raum in E1 zum größeren Raum in B7 verlaufen.

Route $r_{1}$ besitzt in der vorliegenden Menge von Routen die niedrigste Bewertung von 0,62 und ist in Abbildung 4.11a dargestellt. Demnach schlägt der Algorithmus vor, genau dieser Route zu folgen, um die niedrigste Überlastungswahrscheinlichkeit zu erfahren. Die Besonderheit von Route $r_{1}$ ist, dass sie die Halle im Zentrum vollständig vermeidet, indem sie über die Flanke auf der linken Seite verläuft. Darüber hinaus betritt die Route keinen geschlossenen Raum und ist sehr geschmeidig. Die einzige Engstelle, die sie passiert, ist der Eingang zum Zielraum.

Die Routen auf Rang zwei und drei mit einer Bewertung von 0,65 beziehungsweise 0,75 (Abbildung 4.11b) durchlaufen beide den kleinen Raum bei C3/D3, wobei Route $r_{2}$ den Raum zur oberen Seite verlässt und anschließend 
auf der linken Flanke genau wie $r_{1}$ verläuft. Route $r_{3}$ hingegen verlässt den kleinen Raum zur rechten Seite und durchquert kurzzeitig die Halle im Zentrum. Beide Routen betreten wie auch $r_{1}$ kurz darauf den Zielraum durch den Türdurchgang bei A5/A6.

Die auf Rang vier und fünf platzierten Routen (Bewertung von 0,87 und 0,92, Abbildung 4.11c) verwenden nun erstmals die Passage D2/D3 zum Betreten der Halle im Zentrum, um diese im unteren Bereich zu durchlaufen. Route $r_{4}$ durchquert die kreisförmige Installation in der Mitte der Halle, während $r_{5}$ - welche den kürzesten Pfad vom Start zum Ziel darstellt - den bereits eingeschlagenen Weg weiterverfolgt. Beide Routen verwenden erstmals einen anderen Eingang zum Zielraum, nämlich den Gang bei C7.

Schließlich zeigt Abbildung 4.11d diejenigen Routen, denen die höchsten Bewertungswerte mit 0,93 und 1 zugeordnet wurden. Das bedeutet, dass der Algorithmus diesen Routen die potentiell höchste Überlastungswahrscheinlichkeit zuordnet. Route $r_{6}$ verläuft zuerst über die linke Flanke und betritt anschließend die Halle im Zentrum mittels einer schmalen Passage bei C3/C4. Route $r_{7}$ betritt den kleineren Raum bei C3/D3 und verlässt ihn wie auch Route $r_{3}$ auf der rechten Seite. Anschließend durchlaufen sowohl $r_{6}$ als auch $r_{7}$ die Halle im Zentrum in der oberen Region und betreten den Zielraum über die Passage C7.

Zusammengefasst kann gesagt werden, dass der Algorithmus für Routen mit einer möglichst niedrigen Überlastungswahrscheinlichkeit das Vermeiden der Halle im Zentrum, das Verwenden der linken Flanke sowie das Betreten des Zielraums mittels Passage A5/A6 anstelle von $\mathrm{C} 7$ vorschlägt.

\subsubsection{Szenario „Office“}

Das zweite zu diskutierende Szenario namens „Office“ ist erneut eine vereinfachte Version eines realen Gebäudeplans. Abbildung 4.12 zeigt Skizzen der eingegebenen und zu bewertenden Routen, die den Raum unten links bei F1 mit dem Raum oben rechts bei A7/B7 verbinden. Ebenso sind die identifizierten Cluster entsprechend ihrer Bewertung farbig eingezeichnet.

Grundsätzlich können nach Betrachtung der Bewertungsergebnisse die Routen dieses Szenarios in drei Klassen eingeteilt werden. Die erste Klasse besteht aus den Routen $r_{1}-r_{3}$, die alle eine sehr ähnliche, niedrige Bewertung von 0,33, 0,35 und 0,36 besitzen. Alle drei Routen durchlaufen das Gebäude über den langen, horizontalen Flur im unteren Bereich (Zeile E/F) und wenden sich erst spät über den vertikalen Gang bei Spalte 7 dem Zielraum zu. Die Routen unterscheiden sich nur dadurch, dass sie die Räume bei F5-F7 unterschiedlich durchlaufen.

Die Routen $r_{4}$ und $r_{5}$ mit einer Bewertung von 0,57 beziehungsweise 0,63 verwenden zunächst ebenfalls den unteren Bereich des Gebäudeplans, gehen jedoch früher - bei E4/E5 - hoch in Richtung des Zielraums.

Die letzten fünf Routen haben eine ähnlich hohe Bewertung im Bereich von 0,79 und 1 und stellen somit die dritte Gruppe an alternativen Routen dar. 


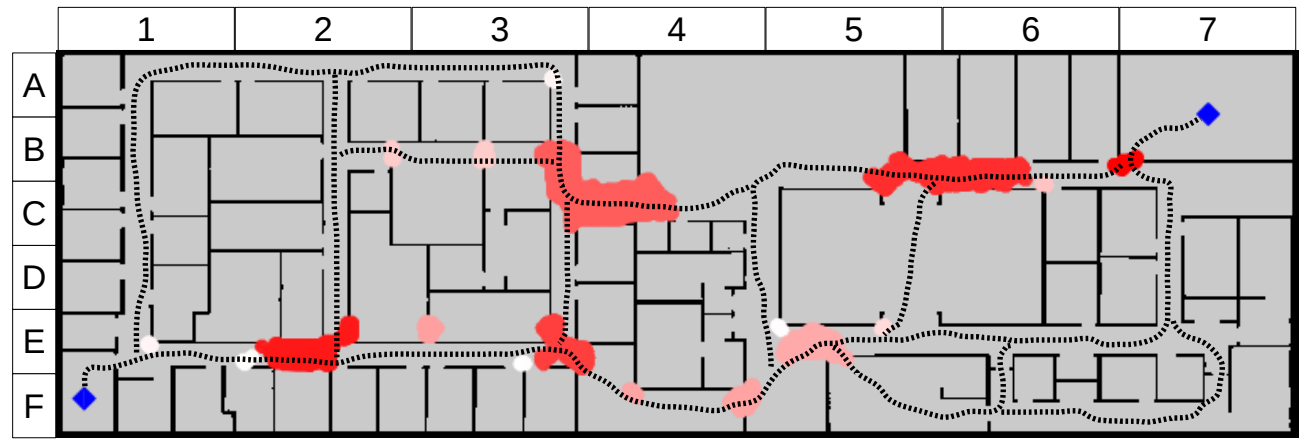

Abbildung 4.12: Skizzen der eingegebenen und bewerteten Routen sowie identifizierte Cluster für das Szenario „Office“.

Die Routen können umschrieben werden als solche, die annähernd die Luftlinie von Start zum Ziel beschreiben und unterschiedliche Variationen im mittleren Bereich des Gebäudeplans besitzen oder den Umweg über A1-A3 gehen.

Zusammengenommen kann festgestellt werden, dass der Algorithmus für potentiell verkehrsarme Routen vorschlägt, den „Hotspot“ bei C3/C4 zu vermeiden, der den oberen westlichen Teil mit dem östlichen Teil verbindet. Außerdem sollte, wenn möglich, der enge Gang bei B5/B6 gemieden werden.

\subsubsection{Zusammenfassende Bewertung}

In diesem Abschnitt wurde ein Algorithmus vorgestellt, der eine Menge von alternativen Routen in Navigationsszenarien innerhalb von Gebäuden bewertet, um eine Rangordnung für die Routen zu erstellen. Dies wird ausschließlich unter der Verwendung eines gewöhnlichen Gebäudeplans erreicht. Die Idee hinter der vorgestellten Bewertungsfunktion ist die Verwendung einer Überlastungswahrscheinlichkeit, die abgeleitet ist von der Annahme, dass wenn ein Punkt innerhalb eines Gebäudes häufig auf einem kürzesten Pfad liegt, dieser ebenfalls häufig von Personen durchlaufen wird.

Der vorgestellte Algorithmus verwendet entsprechend eine Menge von kürzesten Pfaden, um die Überlastungswahrscheinlichkeit zu schätzen, anstelle von komplexen, mikroskopischen Simulationen oder historischen Daten. Wenn es historische Bewegungsdaten von Menschen innerhalb des zu untersuchenden Gebäudes gibt, so können diese als Eingabe für den Algorithmus verwendet werden.

Es sind unterschiedliche Anwendungsfälle denkbar, bei denen die Bewertung von alternativen Routen anhand der Überlastungswahrscheinlichkeit sinnvoll und hilfreich sein kann. So kann zum Beispiel ein Navigationssystem in einem Einkaufszentrum oder in einem Flughafen mit dieser Art der Bewertung proaktiv Engpässe verhindern, in Computerspielen können Nicht-Spieler-Charaktere den menschlichen Spieler mit taktisch ausgewählten alternativen Routen über- 
raschen oder bei der Planung von Gebäuden können potentiell belebte Routen oder Flächen im Vorfeld identifiziert werden, um Benutzerströme zu regulieren oder Schilder und Anzeigen zu platzieren.

Während der Diskussion der Ergebnisse von zwei verschiedenen Szenarien wurde deutlich, dass ein sehr detailliertes Verständnis vom Anwendungsfall benötigt wird, um die Qualität der erzielten Ergebnisse zu bewerten. Um es mit anderen Worten auszudrücken, die resultierenden Bewertungen und Rangfolgen sind sehr allgemein und benötigen eine weitere Interpretation durch menschliche Intuition und persönliche Erfahrungen. Als Beispiel soll eine Bar dienen: Es ist nicht ausreichend, nur das Vermeiden von Türen oder das Durchlaufen von großen Räumen vorzuschlagen. Es hängt ebenfalls stark von der natürlichen Nutzung der Bar ab, nämlich ob Personen im Bereich von Türen stehen oder nicht und, ob Freiflächen durch Personen gefüllt sind, die stehen oder vielleicht laufen, oder eben nicht. Dennoch ist die in diesem Abschnitt vorgeschlagene Bewertungsfunktion ein erster Schritt in Richtung einer quantifizierbaren Qualitätsmetrik für alternative Routen in Navigationsszenarien innerhalb von Gebäuden.

Als zukünftige Arbeit kann die technische Erweiterung des beschriebenen Algorithmus angegangen werden. Die Erstellung der Gewichtskarte kann hinsichtlich anderer Ansätze evaluiert werden als nur das einfache Verdoppeln der Werte. Ebenso könnte bei der Erstellung der zufälligen kürzesten Pfade zusätzlich ein menschliches Bewegungsmodell mit einbezogen werden. Die Konfiguration des Clusterings mittels DBSCAN könnte zudem automatisiert werden, beziehungsweise die Anwendbarkeit weiterer Clusteringverfahren untersucht werden. Der Verfeinerungsschritt der Cluster könnte verbessert werden, indem weitere Informationen berücksichtigt werden als nur die relative Anzahl an alternativen Routen, die den jeweiligen Cluster durchlaufen. Ein Beispiel wäre die Einbeziehung der Fläche des Clusters oder die von den Routen durchlaufenen Fläche. Schließlich kann die eigentliche Bewertung der Routen durch Gewichtungsfaktoren verbessert werden, die den Algorithmus für den jeweiligen Anwender besser anpassbar macht (spezieller Fokus auf Freiflächen oder auf kompakte Stellen wie etwa Türen oder Gänge).

Eine zukünftige Arbeit, die den beschrieben Algorithmus nicht erweitert sondern als Grundlage verwendet, wäre das Abtragen der Überlastungswahrscheinlichkeiten einer Route über die Zeit zwecks Analyse des Verhaltens gegenüber einer andere Route. Indirekt könnte dies als eine Distanz angesehen werden.

\subsection{Archetypische Routen und Archetypische Distanz}

Im vorigen Abschnitt wurden geospatiale Trajektorien verglichen, indem eine Rangfolge erstellt wurde. In diesem Abschnitt wird ein Verfahren vorgestellt, 
bei dem ein Clustering durchgeführt und je ein Repräsentant pro Gruppe zurückgegeben wird. Das verwendete Clusteringverfahren ist die archetypische Analyse, deren grundsätzliche Funktionsweise bereits in Abschnitt 4.2 .2 vorgestellt wurde. Die archetypische Analyse besitzt drei Eigenschaften, die im Umfeld von alternativen Routen in komplexen Umgebungen von Vorteil sind:

1. Die archetypische Analyse führt sogenanntes fuzzy Clustering durch (siehe Abschnitt 4.2.1), weshalb mehrere Repräsentanten pro Gruppe gewählt werden können.

2. Die archetypische Analyse ist ein multidimensionaler Ansatz, weshalb zur Beschreibung der Beobachtungen zahlreiche Merkmale verwendet werden können.

3. Die archetypische Analyse liefert die extremsten Beobachtungen eines Datensatzes zurück. Dies trifft sich mit der grundsätzlichen Motivation dieser Arbeit, möglichst unterschiedliche Routen durch ein Gebäude zu erstellen.

Die weitere Struktur dieses Abschnittes ist wie folgt: zuerst werden drei Archetypen von alternativen Routen definiert (4.4.1). Anschließend wird ein neuartiges Maß für die Ähnlichkeit von Routen definiert, nämlich die archetypische Distanz (4.4.2). Schließlich wird ein System zur Berechnung archetypischer Routen und der archetypischen Distanz vorgestellt, mit der eine Nachbearbeitung einer gegebenen Menge von (alternativen) Routen möglich ist (Abschnitt 4.4.3). Diese archetypischen Routen können gefiltert, analysiert und interpretiert werden, um ein besseres Verständnis für die Beziehung zwischen Routen und Karte zu erhalten.

Zusammengenommen befassen sich die archetypischen Routen und die archetypische Distanz mit zwei der drei in Abschnitt 4.1 beschriebenen Herausforderungen: es werden Alternativen auf extreme Exemplare eingeschränkt und es wird ein Distanzmaß zum Vergleich der Unterschiedlichkeit von Routen vorgestellt.

\subsubsection{Archetypen von alternativen Routen}

Wie bereits in Abschnitt 4.2.2 beschrieben nähert die archetypische Analye die konvexe Hülle der Beobachtungen in einem multidimensionalen Merkmalsraum mit einigen wenigen Punkten an. Diese Punkte werden Archetypen genannt und können als extreme Datenpunkte angesehen werden, die den untersuchten Datensatz beschreiben. Sowohl die Archetypen als auch die Beobachtungen werden durch die konkrete Ausprägung einer Merkmalsmenge repräsentiert. Tabelle 4.1 soll dies verdeutlichen. $\mathrm{Zu}$ sehen ist das Schema einer $100 \times 5$ dimensionalen Matrix $X$, die den zu untersuchenden Datensatz enthält und die Eingabe für die archetypische Analyse ist. Es sind also $N=100$ Beobachtungen 


\begin{tabular}{|c|c|c|c|c|c|}
\hline & $m_{1}$ & $m_{2}$ & $m_{3}$ & $m_{4}$ & $m_{5}$ \\
\hline$r_{1}$ & & & & & \\
\hline$r_{2}$ & & & & & \\
\hline$\vdots$ & & & & & \\
\hline$r_{100}$ & & & & & \\
\hline
\end{tabular}

Tabelle 4.1: Schematische Darstellung der $N \times m$-dimensionalen Matrix $X$, die den zu untersuchenden Datensatz enthält und als Eingabe für die archetypische Analyse dient. In diesem Beispiel entspricht jede der 100 Zeilen eine Route und jede Route ist durch fünf Merkmale dargestellt.

(zum Beispiel Routen) eingetragen mit jeweils $m=5$ Merkmalen. Nun gilt es, daraus Archetypen zu extrahieren.

Im Folgenden werden drei unterschiedliche Arten von Archetypen von alternativen Routen definiert: die abstrakten archetypischen Routen, die realisierten archetypischen Routen und die treuen archetypischen Routen.

Abstrakte archetypische Routen Eine konkrete Ausprägung einer Merkmalsmenge wird abstrakte archetypische Route genannt. Dieser Punkt im mehrdimensionalen Merkmalsraum, der äquivalent zu der ursprünglichen Beschreibung von Archetypen aus [36] ist, muss nicht notwendigerweise beobachtet worden sein. Abbildung 4.13 zeigt schematisch drei abstrakte archetypische Routen mitsamt der Ausprägungen der Merkmalsmenge. Die Grafik nimmt Bezug auf das Schema des Datensatzes aus Tabelle 4.1 und zeigt einen Archetypen $A_{1}$, bei dem die Ausprägung der Merkmale $m_{1}$ und $m_{5}$ sehr hoch sind und die anderen drei Merkmalsausprägungen moderat. Archetyp $A_{2}$ besitzt hohe Ausprägungen in allen fünf Merkmalen und Archetyp $A_{3}$ sehr niedrige. Laut der archtypischen Analyse würden diese drei Archetypen den gegebenen, fiktiven Datensatz gut beschreiben.

Realisierte archetypische Routen Elemente eines untersuchten Datensatzes, das heißt konkrete Beobachtungen im Sinne von alternativen Routen, sind ebenso wie abstrakte archetypische Routen Punkte in einem multidimensionalen Merkmalsraum. Demnach wird ein möglicherweise nicht beobachteter abstrakter Archetyp durch die Auswahl eines Repräsentanten „realisiert“, wodurch diese realisierte archetypische Route eine konkrete Beobachtung sowohl im Merkmals- als auch im Datenraum ist. Im Zuge dieser Arbeit wird für die Auswahl eines Repräsentanten eine einfache Nächste-Nachbarn-Suche vorgeschlagen, die sich auf die Koeffizientenmatrix $\alpha$ bezieht. Wie bereits in Abschnitt 4.2.2 erläutert, nähert die archetypische Analyse jede Beobachtung durch das Produkt der Koeffizientenmatrix $\alpha$ und der Archetypen-Matrix $Z$ an. Jede Beobachtung ist also eine konvexe Kombination der Archetypen. Es 


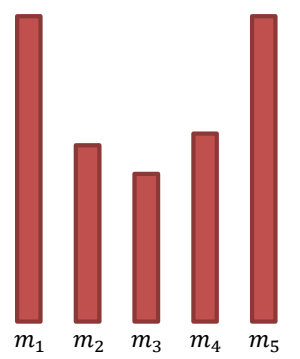

$A_{1}$

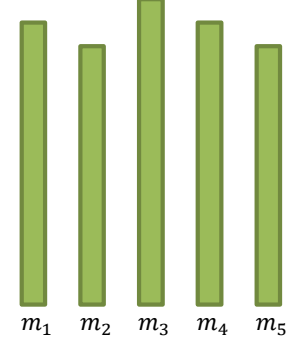

$A_{2}$

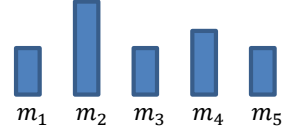

$A_{3}$

Abbildung 4.13: Schematische Darstellung von drei ermittelten abstrakten archetypischen Routen mit der jeweiligen Ausprägung der Merkmalsmenge.

wird nun definiert, dass eine realisierte archetypische Route diejenige Beobachtung ist, die in der entsprechenden Spalte der Koeffizientenmatrix $\alpha$ einen Maximalwert besitzt. Abbildung 4.14 zeigt einen beispielhaften Datensatz im Merkmalsraum, wobei die eingezeichneten Punkte die Beobachtungen sowie die drei abstrakten Archetypen darstellen. Diejenige Beobachtung, die am nächsten zu einem Archetypen liegt, wird als realisierte archetypische Route gewählt (hier: $p$ bei $A_{1}$ ).

Treue archetypische Routen Eine treue archetypische Route ist definiert als eine konkrete Beobachtung, die exakt dieselbe Ausprägung der Merkmalsmenge wie eine berechnete abstrakte archetypische Route besitzt. Dies kann auf zwei unterschiedlichen Wegen zustandekommen. Zum einen kann eine konkrete Beobachtung identisch mit einer abstrakten archetypischen Route sein. Die Tatsache, dass die Beobachtung einen entsprechenden $\alpha$-Wert von genau 1 besitzt, macht sie gleichzeitig zu einer treuen archetypischen Route. Zum anderen kann der Fall eintreten, dass ein Datensatz keine treue archetypische Route besitzt, weshalb diese synthetisiert werden müsste. Die Klasse der synthetisch hergestellten treuen archetypischen Routen ist insbesondere deshalb interessant, da der Fehler, der durch die Realisierung eines abstrakten Archetypen durch eine Nächste-Nachbarn-Suche eingeführt wird, gleich Null sein würde. Die Forschungsfrage der Erstellung von Routen mit vordefinierten Eigenschaften ist sehr interessant, jedoch bislang ungelöst und wird für zukünftige Arbeiten an dieser Stelle unbeantwortet gelassen.

Die abstrakten, realisierten und treuen archetypischen Routen wurden in diesem Abschnitt schematisch beschrieben. Die zur Berechnung der Routen verwendeten Merkmale sowie das eigentliche Vorgehen werden in Abschnitt 4.4.3 beschrieben, im dem das System zur Berechnung archetypischer Routen vorgestellt wird. In der Evaluation (Abschnitt 4.4.4) werden auch die Archetypen im Datenraum vorgestellt, sprich als konkrete Routen innerhalb einer 


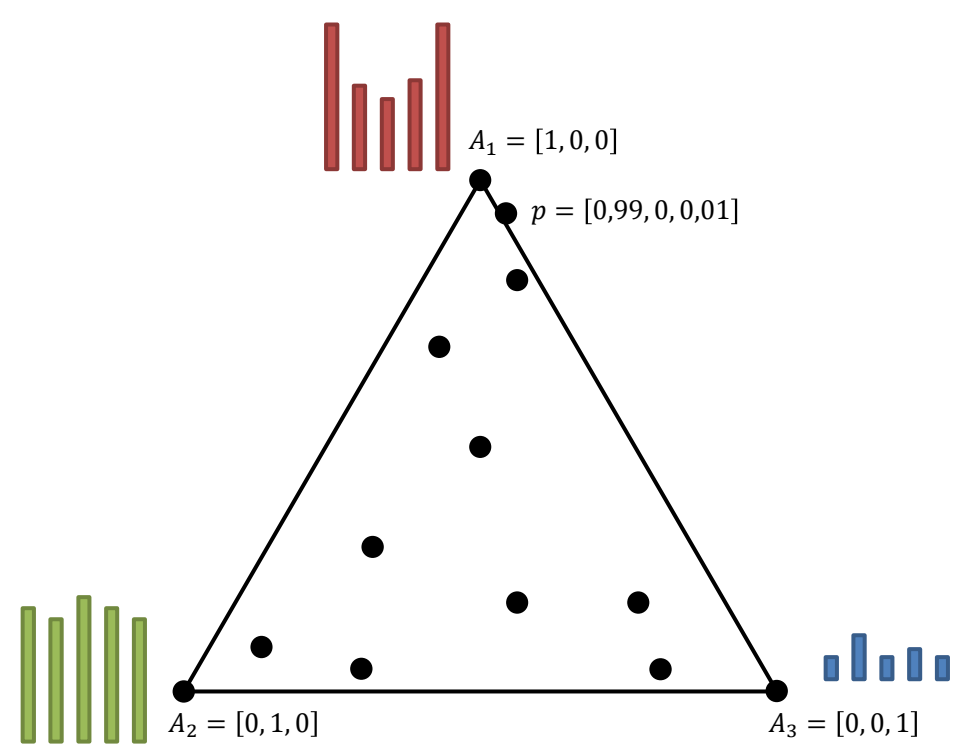

Abbildung 4.14: Schematische Darstellung zur Realisierung von abstrakten archetypischen Routen. Zu sehen sind der Datensatz (schwarze Punkte), die ermittelten Archetypen $\left(A_{1}-A_{3}\right)$, schematisch deren Merkmalsausprägungen sowie die realisierte archetypische Route $p$ für Archetyp $A_{1}$.

Karte.

\subsubsection{Archetypische Distanz als Distanzmaß für Trajektorien}

Mithilfe der archetypischen Analyse kann ein Datensatz durch eine Menge von abstrakten Archetypen zusammengefasst werden, die aus konkreten Merkmalsausprägungen von extremen Beobachtungen bestehen. Dies erlaubt die direkte Berechnung der Ähnlichkeit zwischen Beobachtungen und Archetypen im Merkmalsraum (nicht im Datenraum) anhand der Euklidischen Norm der Merkmalsunterschiede.

Archetypische Distanz Die archetypische Distanz - im Kontext der vorliegenden Arbeit zwischen zwei geospatialen Trajektorien - ist definiert als deren Distanz im Merkmalsraum. Demnach existiert diese Distanz auch zwischen abstrakten Archetypen und kann dazu verwendet werden, eingebettete Archetypen in unterschiedlichen Geometrien auszurichten. Um die Ergebnisse einer archetypischen Analyse zu visualisieren ist es beispielsweise möglich, eine Distanzmatrix für die Merkmalsvektoren aller Archetypen und aller realen Beobachtungen zu erstellen und anschließend multidimensionale Skalierung [42] anzuwenden, um die Punkte derart einzubetten, dass Ähnlichkeit und Unähn- 
lichkeit möglichst gut erhalten bleiben.

Als Folge dessen kann die archetypische Distanz zu einer Euklidischen Distanz zwischen Beobachtungen reduziert werden, indem die Beobachtungen beispielsweise mittels Techniken der multidimensionalen Skalierung in einen niedrigdimensionalen Euklidischen Raum eingebettet werden. Dies ist insbesondere dann interessant, wenn Beobachtungen, die während der durchgeführten archetypischen Analyse noch nicht vorhanden waren, untersucht werden sollen. Diese können mittels Lateration einfach in die niedrigdimensionale Einbettung eingezeichnet werden.

Das Konzept der archetypischen Distanz ist auch konform zum Konzept der realisierten archetypischen Routen. Wenn anstatt einer einzigen Route eine Menge von Routen realisiert wird (beispielsweise die $k$-nächsten-Nachbarn oder alle Beobachtungen mit einem $\alpha$-Wert größer einem Schwellwert), so besitzen alle realisierten archetypischen Routen eine kleine archetypische Distanz zueinander.

Dies wurde bereits indirekt in Abbildung 4.14 visualisiert. Die fünfdimensionalen Beobachtungen wurden in 2D dargestellt und Beobachtung $p$ hat eine niedrige archetypische Distanz zum Archetypen $A_{1}$. Wenn in dem Datensatz, der Abbildung 4.14 zugrunde liegt, eine neue Beobachtung hinzugefügt wird, so kann diese in die Darstellung integriert werden. Das Konzept der archetypischen Distanz sowie die Entsprechung im Datenraum - also die Routen innerhalb einer Karte - werden im entsprechenden Abschnitt der Evaluation weiter diskutiert.

\subsubsection{System zur Berechnung archetypischer Routen}

In diesem Abschnitt werden ein System zur Berechnung archetypischer Routen sowie deren verwendete Merkmale beschrieben. Das System wird als Nachweis der Machbarkeit der Definitionen der archetypischen Routen und der archetypischen Distanz verwendet. Dem im Folgenden beschriebenen und in [77] veröffentlichten System liegt ein Demonstrator zugrunde, der in einer studentischen Arbeit [101] entwickelt wurde und die Verwendung der archetypischen Analyse untersuchen soll.

\subsubsection{Konzept und Implementierung}

Das System zur Berechnung archetypischer Routen wurde vollständig in R [114] implementiert, einer Programmiersprache zur Durchführung und Visualisierung statistischer Berechnungen und Analyse von Daten.

Die Eingabe in das System ist ein Gebäudeplan in der Form einer gewöhnlichen Bitmap, wobei weiße Pixel begehbare Flächen repräsentieren und schwarze Pixel entsprechend Hindernisse wie Wände oder Möbel. Eine weitere Eingabe ist eine Menge von Routen mit denselben Start- und Zielpunkten, die mittels des Penalty-Algorithmus (siehe Abschnitt 3.3.2) erstellt wurden. Diese Menge wird durch einen Vektor bestehend aus den Tupeln $<x, y$, rid, cid $>$ 
dargestellt. $x$ und $y$ repräsentieren die Koordinaten eines Punktes einer Route und rid ordnet die Punkte den Routen zu. cid ordnet eine Route einer Äquivalenzklasse zu, die durch das topologische Konzept der Homotopie definiert wurde (siehe Abschnitt 3.2). Demnach sind zwei Routen mit demselben Wert für cid homotop zueinander und können als äquivalent angesehen werden, während zwei Routen mit unterschiedlichen Werten für cid nicht homotop zueinander sind und deshalb als alternative Routen gelten. Die Homotopieklasse wird für die Analyse der Ausdrucksfähigkeit des Systems verwendet und nicht zur Berechnung der Archetypen selbst.

Die Durchführung der eigentlichen archetypischen Analyse wurde mittels des R-Pakets ,archetypes“ [70] vorgenommen, was mittels CRAN, einem zentralen Archiv für R-Pakete, bezogen werden kann. Das R-Paket wurde für die Erstellung der meisten Abbildungen in diesem Abschnitt verwendet.

\subsubsection{Ausgewählte Merkmale}

Um die archetypische Analyse im Kontext von alternativen Routen anwenden zu können, muss eine Merkmalsmenge definiert werden, die gegebene Routen als einen numerischen Vektor darstellen kann. Im Folgenden wird eine wenig komplexe und intuitive Merkmalsmenge beschrieben, die innerhalb der durchgeführten Experimente eine gute Beschreibungskraft zeigt. Die Liste ist jedoch weder vollständig noch sollte sie ohne vorherige Überprüfung auf andere Domänen übertragen werden. Dies ist der Tatsache geschuldet, dass die Darstellung einer Route durch einen Vektor von Realzahlen nur eine fallbezogene Annäherung darstellt. Aus diesem Grunde ist die im Folgenden beschriebene Merkmalsmenge nur eine Variante, die mit relativ wenig Aufwand zum gewünschten Ergebnis führt.

Ein Merkmal wird durch eine standardisierte Fließkommazahl beschrieben, die eine Eigenschaft der Route, wie beispielsweise deren Länge, repräsentiert. Für jede alternative Route wird dieselbe Menge von deskriptiven Merkmalen extrahiert, die in einem $m$-dimensionalen Vektor gespeichert wird. Diese Zeilenvektoren bilden Matrix $X$ innerhalb der archetypischen Analyse (siehe Abschnitt 4.2.2).

Die im Folgenden verwendeten Merkmale können grundsätzlich in drei Gruppen eingeteilt werden: Merkmale einer Route bezüglich ihrer geometrischen Form, bezüglich ihrer Beziehung zum kürzesten Pfad sowie bezüglich ihrer Beziehung zum Gebäudeplan.

\section{Routenmerkmale bezüglich der geometrischen Form}

- chull_area: Der Flächeninhalt des Polygons, das durch die konvexe Hülle der Punkte einer Route aufgespannt wird.

- chull_size: Die Anzahl der Punkte, die die konvexe Hülle der Punkte einer $\bar{R}$ oute definiert. 
- chull_centroid_x/y: Die $x$ - und $y$-Koordinaten des geometrischen Schwerpunkts des Polygons, das durch die konvexe Hülle der Punkte einer Route aufgespannt wird.

- length: Die absolute Länge der Route, gemessen in der Anzahl der Punkte der Route.

- angularsum_cancelling/positive: Die summierten Winkelwerte der Richtungswechsel einer Route. Da eine Route durch pixelweise Koordinaten dargestellt ist, existieren die Winkel der Richtungswechsel in 45-Grad-Schritten in den Intervallen $\left[-180^{\circ},+180^{\circ}\right]$ (cancelling) und $\left[0^{\circ}, 360^{\circ}\right]$ (positive).

\section{Routenmerkmale bezüglich des kürzesten Pfades}

- relative length: Die absolute Länge der Route (siehe Merkmal length) dividiert $\bar{d}$ urch die absolute Länge der kürzesten Route.

- dtw: Die Distanz zwischen der Route und der kürzesten Route, die mittels Dynamic Time Warping (DTW) berechnet wurde. DTW ist ein Algorithmus für das Erfassen der Ähnlichkeit zwischen zwei Trajektorien (siehe Abschnitt 4.2.3).

\section{Routenmerkmale bezüglich des Gebäudeplans}

- average/min_heat: Dieses Merkmal bezieht sich auf die Gewichtskarte, die zur Berechnung der Überlastungswahrscheinlichkeit (siehe Abschnitt 4.3) erstellt wurde. Demnach bezeichnet die durchschnittliche „Hitze“ einer Route die Summe der Werte der Gewichtskarte aller Punkte einer Route dividiert durch die absolute Länge der Route. Das Minimum der Hitze ist entsprechend der kleinste Wert der Gewichtskarte der Punkte einer Route.

Die Extraktion der soeben beschriebenen Merkmale wird ausschließlich mit einfachen Standardwerkzeugen von $\mathrm{R}$ durchgeführt. Allein für die Berechnung der DTW-Distanz wird eine bestehende Bibliothek verwendet, die mittels CRAN zu beziehen ist [93].

\subsubsection{Evaluation}

In diesem Abschnitt werden ausführliche Experimente unter Verwendung unterschiedlicher Gebäudepläne durchgeführt. Die Beschreibung folgt dem grundsätzlichen Ablauf der archetypischen Analyse und diskutiert insbesondere Auffälligkeiten. 


\subsubsection{Aufbau der Experimente}

Die Evaluation des Systems zur Berechnung archetypischer Routen und der archetypischen Distanz findet unter Verwendung von vier unterschiedlichen Gebäudeplänen statt. Szenario „Office“ zeigt einen sehr regelmäßigen Gebäudeplan mit rechteckigen Räumen und Gängen, während Szenario „Spa“ einen eher runden Gebäudeplan darstellt, wobei der Fokus des Plans in seiner Mitte liegt. Szenario „Doom“ stellt eine vereinfachte Version einer Karte dar, die in einem Ego-Shooter verwendet wird, und besitzt eine Vielzahl an unregelmäßigen Wegen. Schließlich repräsentiert das Szenario „White House“ eine vereinfachte Version eines historischen Gebäudeplans des Weißen Hauses.

Für jede der untersuchten Karten wurden 400 Routen mithilfe des PenaltyAlgorithmus (siehe Abschnitt 3.3.2) erstellt. Jede Route wird genau einer Äquivalenzklasse zugeordnet, die durch das Konzept der Homotopie definiert ist (siehe Abschnitt 3.2). So sind die 400 Routen im Szenario „Office“ in insgesamt 23 unterschiedliche Homotopieklassen eingeteilt, das Szenario "Spa“ hat 12 Klassen, Szenario „Doom“ 10 Klassen und „White House“ 37 Klassen. Die Anzahl der Homotopieklassen hängt naturgemäß von der verwendeten Karte, aber auch der Anzahl der erstellten Routen ab: Je mehr Routen erstellt werden, desto höher ist die Chance, eine neue Homotopieklasse zu finden.

Für jedes Szenario wurden mehrere Durchläufe der archetypischen Analyse mit unterschiedlichen Werten für $k$ (Anzahl Archetypen, siehe Abschnitt 4.2.2) im Bereich von 1 bis 10 durchgeführt, um unterschiedliche Kombinationen von Archetypen zu identifizieren und zu diskutieren. Für jedes $k$ wurde die archetypische Analyse mehrfach durchgeführt, um lokale Minima auszuschließen.

\subsubsection{Berechnung abstrakter Archetypen}

Zunächst soll Szenario „Office“ dazu dienen, die grundlegende Funktionsweise des Systems zur Berechnung archetypischer Routen zu erläutern und erste Ergebnisse bewerten zu können. Die archetypische Analyse nähert die konvexe Hülle der Beobachtungen im Merkmalsraum an, weshalb die Residuenquadratsumme (residual sum of squares, $R S S$ ) dafür verwendet werden kann, die möglichst optimale Anzahl an Archetypen $k$ im Vorfeld abzuschätzen. Ein Abflachen des Eigenwertediagramms der $R S S$ deutet auf einen angemessenen Wert für $k$ hin, da das Hinzufügen eines zusätzlichen Archetyps wenig Einfluss auf die Verminderung des Annäherungsfehlers hat. Diese Methode wird auch Scree-Test oder „Ellbogenkriterium“ genannt (siehe [69] sowie Abschnitt 4.2.2).

In Abbildung 4.15 sind für das Szenario „Office“ die $R S S$-Werte für unterschiedliche Werte von $k$ eingezeichnet. Es ist offensichtlich, dass der $R S S$-Wert sowohl von $k=1$ auf $k=2$,abfällt" als auch von $k=2$ auf $k=3$. Die Differenz zwischen $k=3$ und $k=4$ ist jedoch nur marginal, weshalb im weiteren Verlauf die Anzahl der Archetypen auf $k=3$ gesetzt wird. Um bei der Annäherung der konvexen Hülle ein lokales Minimum zu vermeiden, wird die archetypische Analyse mehrfach ausgeführt und entsprechend das beste Modell näher 


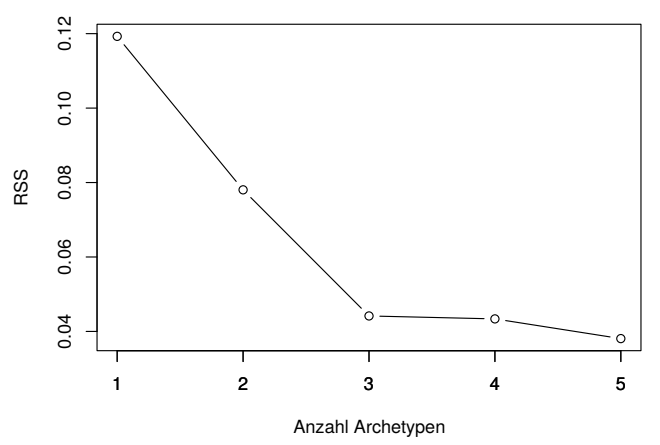

Abbildung 4.15: Eigenwertediagramm der resultierenden $R S S$ für unterschiedliche Werte von $k$ im Szenario „Office“. Bei $k=3$ ist ein Abflachen der Kurve zu erkennen.

untersucht.

Eine geeignete Repräsentation für Archetypen ist ein Säulendiagramm, wobei eine Säule eine konkrete Merkmalsausprägung darstellt. Abbildung 4.16 zeigt demnach die drei ermittelten abstrakten archetypischen Routen mit den elf in Abschnitt 4.4.3 vorgestellten Merkmalen.

Nach der Berechnung der Archetypen folgt nun die Interpretation selbiger. Archetyp A1, dargestellt in der oberen Zeile von Abbildung 4.16, besitzt niedrige Werte für die Anzahl der Punkte der konvexen Hülle (chull_size) sowie für deren Flächeninhalt (chull_area). Das bedeutet, dass eine Route, die dem Archetyp $A 1$ entspricht, eher geradlinig ist und ungefähr in Luftlinie vom Start zum Ziel verläuft. Die moderaten Werte für die Koordinaten des Schwerpunkts der konvexen Hülle (chull_centroid_ $x / y$ ) deuten darauf hin, dass der Hauptteil einer solchen Route die Karte vergleichsweise mittig durchläuft. Niedrige Werte für die absolute Länge (length), die Länge in Bezug zur kürzesten Route (relative_length), die summierten Winkelwerte der Richtungswechsel (angularsum_cancelling/positive) sowie die DTW-Distanz zur kürzesten Route (dtw) weisen darauf hin, dass eine Route, die dem Archetyp $A 1$ ähnelt, kurz sein wird. Archetyp A2 (mittlere Zeile in Abbildung 4.16) besitzt hohe bis sehr hohe Werte für alle Merkmale ausgenommen bezüglich der „Hitze“. Das bedeutet, dass eine entsprechende Route sich eher wie ein Umweg verhält: sie ist verhältnismäßig lang, besitzt viele Richtungswechsel und durchläuft den Gebäudeplan eher im unteren Bereich (der Koordinatenursprung befindet sich in der oberen linken Ecke des Gebäudeplans). Archetyp $A 3$ besitzt schließlich hohe bis sehr hohe Werte für die Merkmale, die eine recht lange Route vermuten lassen (Größe und Fläche der konvexen Hülle, absolute und relative Länge, Richtungswechsel und Distanz zur kürzesten Route). Der niedrige Wert für den Schwerpunkt der konvexen Hülle lässt darauf schließen, dass die entsprechende Route potentiell den oberen linken Teil des Gebäudeplans durchläuft. 


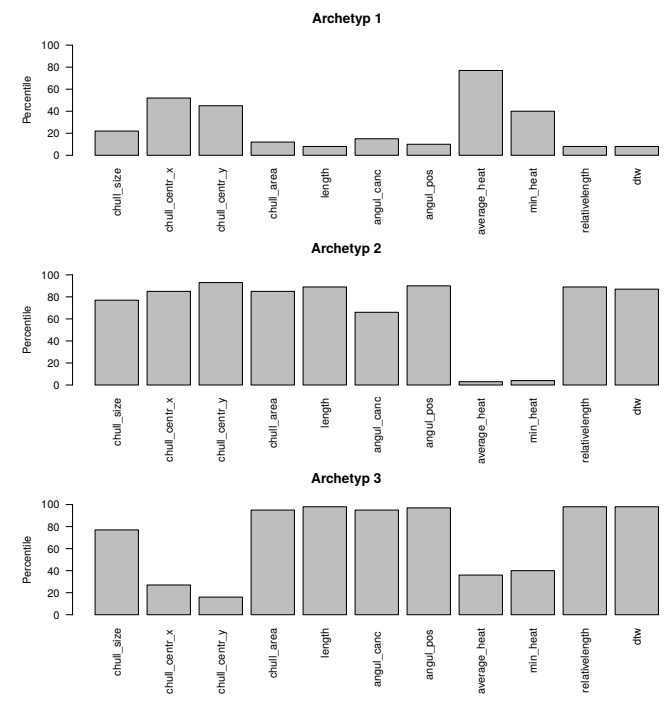

Abbildung 4.16: Drei abstrakte archetypische Routen als Säulendiagramme für das Szenario „Office“.

\subsubsection{Realisierte Archetypen mittels Nächster-Nachbar-Suche}

Nach der Berechnung der abstrakten archetypischen Routen folgt nun die Realisierung selbiger. Dadurch verändert sich die Sichtweise von Merkmalsausprägungen in Form von Säulendiagrammen zu konkreten Beobachtungen im Datenraum. Dies ermöglicht die Validierung der vorläufig durchgeführten Interpretation sowie die Beurteilung, ob die Interpretationen zu den berechneten Archetypen passen.

Wie in Abschnitt 4.2.2 erläutert, werden Beobachtungen mithilfe der Koeffizientenmatrix $\alpha$ als konvexe Kompositionen aus unterschiedlichen Archetypen dargestellt. Abbildung 4.17 zeigt ein Dreiecksdiagramm, bei dem die Eckpunkte die drei abstrakten Archetypen aus Abbildung 4.16 darstellen. Die übrigen eingezeichneten Punkte repräsentieren den Datensatz, also die Beobachtungen. Ein Dreiecksdiagramm eignet sich sehr gut zur Darstellung einer konstanten Summe, in unserem Falle aufgrund der konvexen Komposition der Summe 1. Demnach ist beispielsweise Archetyp $A 1$ ausschließlich aus sich selbst komponiert; eine Beobachtung, die auf der Dreiecksseite zwischen $A 1$ und $A 2$ liegt, ist entsprechend eine Komposition aus diesen beiden Archetypen, nicht aber aus A3. Aus den paarweisen Abständen zwischen den Datenpunkten ist ersichtlich, dass Beobachtungen existieren, die sehr nah an einem bestimmten Archetypen liegen. Wie in Abschnitt 4.4.1 erläutert wird nun für jeden Archetypen eine einfache Nächster-Nachbar-Suche basierend auf den korrespondierenden $\alpha$-Wert durchgeführt, um eine konkrete Beobachtung auszuwählen, welche die realisierte archetypische Route darstellt. Demnach werden die abstrakten archetypischen Routen als Punkte im Merkmalsraum verlassen und es werden realisierte archetypische Routen als konkrete Beobachtungen im Datenraum 


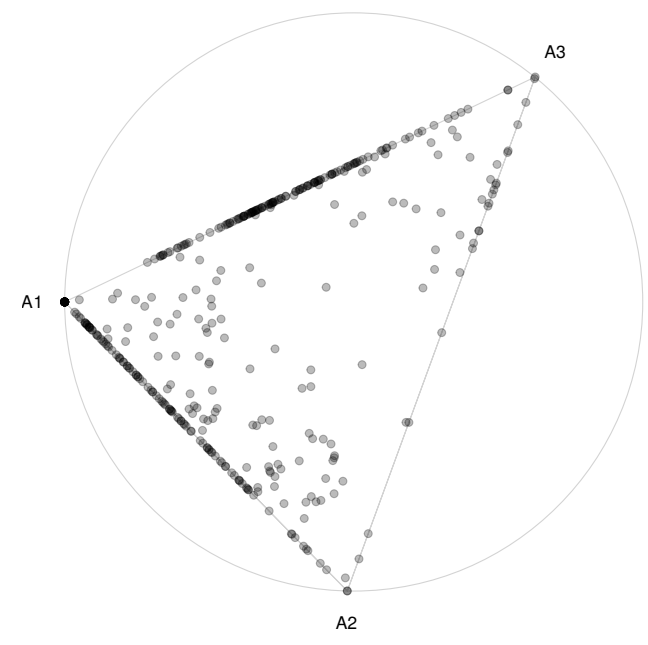

Abbildung 4.17: Dreiecksdiagramm, das die Abstände zwischen den Archetypen und den Beobachtungen im Merkmalsraum für $k=3 \mathrm{im}$ Szenario „Office“ zeigt.

behandelt.

Abbildung 4.18 stellt die Realisierung der drei abstrakten archetypischen Routen aus Abbildung 4.16 mittels Nächster-Nachbar-Suche dar. Es ist ersichtlich, dass die Interpretation der Merkmalsausprägungen basierend auf den abstrakten archetypischen Routen aus Abschnitt 4.4.4.2 durchaus zu den gewählten Repräsentanten passt. Die geradlinig und mittig verlaufende schwarze Route korrespondiert mit Archetyp A1. Die im unteren Bereich verlaufende rote Route mit vielen Richtungswechseln stellt Archetyp A2 dar und die sehr lange, eher im oberen Bereich verlaufende grüne Route ist entsprechend Archetyp A3. Dieses Ergebnis zeigt, dass das vorgestellte Verfahren zum Filtern eines Datensatzes bestehend aus 400 Routen eingesetzt werden kann, sodass die drei realisierten archetypischen Routen angemessene alternative Routen für das gegebene Navigationsszenario darstellen.

\subsubsection{Verteilung der $\alpha$-Werte vs. „Ellbogenkriterium“}

In der Literatur zur archetypischen Analyse wird häufig das „Ellbogenkriterium" in Kombination mit dem Eigenwertediagramm der $R S S$ verwendet, um einen passenden Wert für die Anzahl der Archetypen $k$ zu fixieren. In der vorliegenden Evaluation wurde dieses Vorgehen auch für das Szenario „Office“ verwendet. Während der Durchführung der Experimente wurde jedoch ersichtlich, dass die Berücksichtigung der Verteilung der Werte der Koeffizientenmatrix $\alpha$ helfen kann, einen angemessenen Wert für $k$ zu finden. Diese Erkenntnis soll im Folgenden mittels des Szenarios „Spa“ demonstriert werden.

In Abbildung 4.19 ist das Eigenwertediagramm der $R S S$ für das Szenario „Spa“ dargestellt. Es ist offensichtlich, dass es eine starke Abflachung der Kurve 


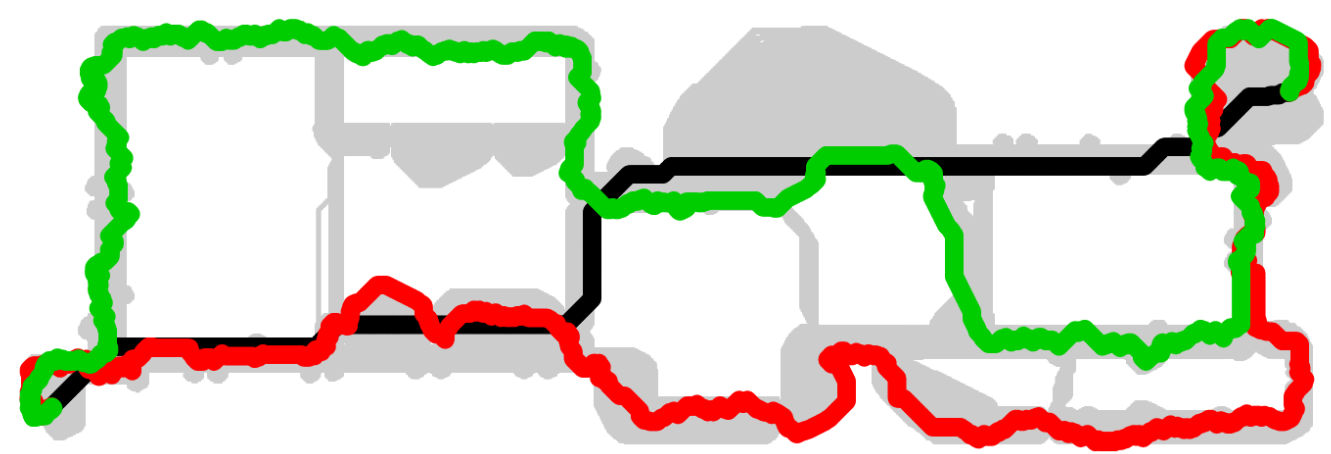

Abbildung 4.18: Drei realisierte archetypische Routen im Szenario „Office“ (rot, grün, schwarz). Die grauen Flächen ergeben sich aus den 400 Routen, die als Eingabe dienen. Wände und Hindernisse wurden für eine bessere Betrachtung der Abbildung ausgelassen.

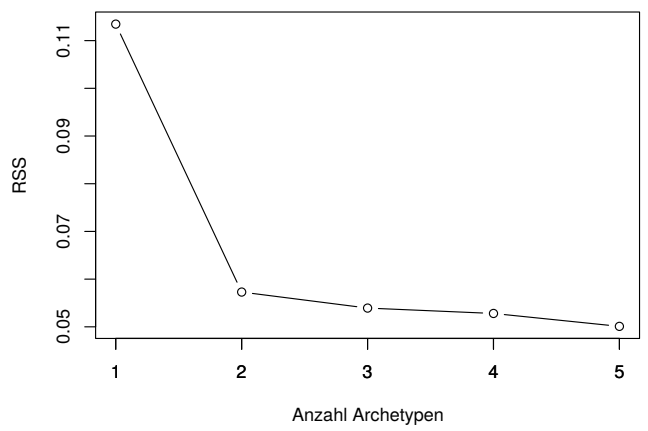

Abbildung 4.19: Eigenwertediagramm der resultierenden $R S S$ für unterschiedliche Werte von $k$ im Szenario „Spa“. Bei $k=2$ ist ein Abflachen der Kurve zu erkennen.

bei $k=2$ gibt, was bedeutet, dass die Verwendung von mehr als zwei Archetypen nur eine relativ kleine Verminderung des Fehlers bei der Annäherung der konvexen Hülle ergibt.

Abbildung 4.20 zeigt Boxplot-Diagramme zur Darstellung der Werteverteilung der Koeffizientenmatrix $\alpha$. Für ein gegebenes $k$ gibt es demnach $k$ BoxplotDiagramme, wobei sich beispielsweise Boxplot-Diagramm 1 auf die erste Spalte der Koeffizientenmatrix $\alpha$ - und somit auf Archetyp $A 1$ - bezieht und die entsprechenden Streuungs- und Lagemaße graphisch darstellt. Abbildung 4.20a bestätigt, dass $k=2$ eine gute Wahl für das gegebene Szenario ist: Die Maxima beider Boxplot-Diagramme liegen extrem hoch (1 beziehungsweise 0,9996), was bedeutet, dass die Differenz zwischen den berechneten abstrakten und realisierten archetypischen Routen sehr gering ist. Außerdem ist ersichtlich, dass dies kein Einzelfall ist, denn 25\% der Werte liegen oberhalb von etwa 0,7 (oberes Quartil). Anders als die Abflachung im $R S S$-Eigenwertediagramm vermu- 


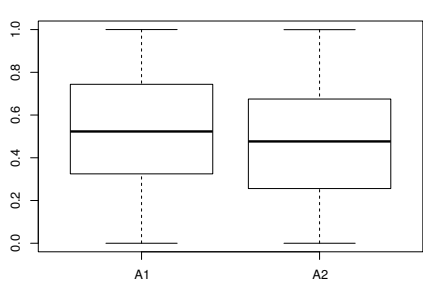

(a) $k=2$

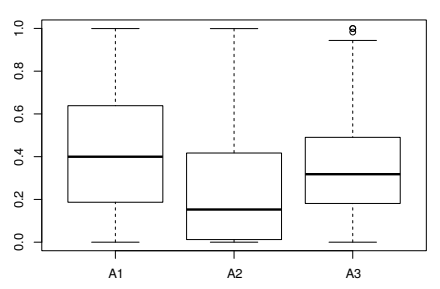

(b) $k=3$

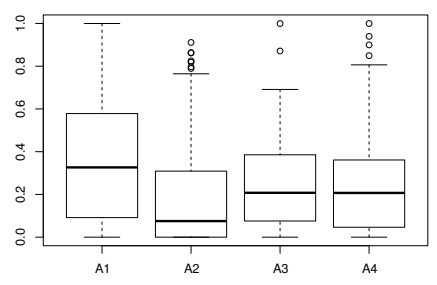

(c) $k=4$

Abbildung 4.20: Boxplot-Diagramme zur Darstellung der Werteverteilung der Koeffizientenmatrix $\alpha$ für verschiedene Werte von $k$ im Szenario ,Spa“.

ten lässt, scheint $k=3$ ebenfalls eine gute Wahl für die Anzahl der Archetypen zu sein (siehe Abbildung 4.20b). Alle drei Boxplot-Diagramme besitzen sehr hohe Maxima (0,9993, 0,9991 und 0,9996), und auch die oberen Quartile liegen weit oben (etwa 0,64 für Archetyp $A 1$ und etwa 0,4 beziehungsweise 0,45 für die Archetypen $A 2$ und $A 3$ ). Die Boxplot-Diagramme für $k=4$ (Abbildung $4.20 \mathrm{c})$ zeichnen jedoch ein anderes Bild: die Endpunkte der oberen Antennen liegen bei den Archetypen $A 2, A 3$ und $A 4$ deutlich unter 1 und es existieren mehrere Ausreißer nach oben. Damit werden diese Archetypen durch Sonderfälle beschrieben.

Um die Vermutung der Eignung von $k=3 \mathrm{zu}$ verifizieren, werden in Abbildung 4.21 die realisierten archetypischen Routen für unterschiedliche Werte von $k$ dargestellt. Das Ergebnis für $k=2$ (Empfehlung des Ellbogenkriteriums) zeigt eine sehr geradlinige Route, die den unteren Teil des Gebäudeplans durchläuft sowie eine recht gewundene Route im oberen Bereich (Abbildung 4.21a). Das Ergebnis für $k=3$ (alternative Empfehlung basierend auf der Verteilung der $\alpha$-Werte) zeigt zwei Routen mit denselben beschriebenen Eigenschaften wie bei $k=2$ sowie eine weitere Route, die verhältnismäßig mittig durch den Gebäudeplan verläuft (Abbildung 4.21b). Mit variierenden Werten für $k$ bleiben die identifizierten Archetypen nicht notwendigerweise identisch, da die Lösungen nicht voneinander abhängen [150]. Abbildung 4.21c zeigt schließlich die realisierten archetypischen Routen für $k=4$, wobei drei der vier Routen denselben Verlauf wie die Routen im Falle von $k=3$ besitzen. Die neu hinzugekommene Route ist auf den ersten Blick recht ähnlich zu der im oberen Bereich verlaufenden Route, und sie teilen sich tatsächlich dieselbe Homotopieklasse (grüne und blaue Route).

Es ist also ersichtlich, dass nicht nur der vom Ellbogenkriterium vorgeschlagene Wert $k=2 \mathrm{zu}$ angemessenen alternativen Routen führt, sondern auch $k=3$, was durch die Inspektion der Koeffizientenverteilung nachvollziehbar wurde. Lediglich ab $k=4$ enthalten die Ergebnisse Routen, die basierend auf ihrem Verlauf und der zugeordneten Homotopieklassen doppelt und demnach überflüssig sind.

Die im ersten Moment als Nachteil wahrgenommene Tatsache, dass ab einem 


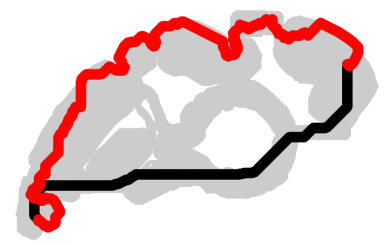

(a) $k=2$

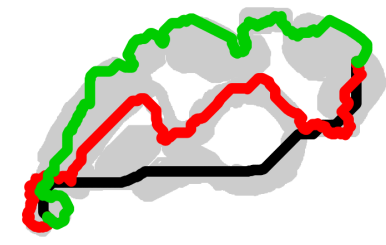

(b) $k=3$

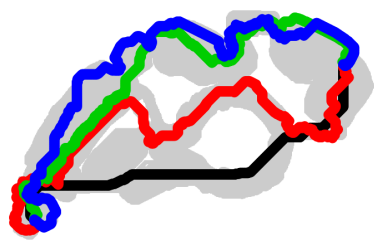

(c) $k=4$

Abbildung 4.21: Realisierte archetypische Routen für unterschiedliche Werte von $k$ im Szenario „Spa“ (rot, schwarz, grün, blau). Die grauen Routen im Hintergrund stellen die komplette Eingabemenge der 400 Routen dar. Wände und Hindernisse wurden für eine bessere Betrachtung der Abbildungen ausgelassen.

bestimmten Wert von $k$ zueinander homotope und demnach äquivalente Routen gefunden werden, kann aber auch als Vorteil aufgefasst werden. Anstelle die Anzahl der Archetypen $k$ zu beschränken, kann dieser Wert variabel gehalten und ein zusätzlicher Homotopie-Test durchgeführt werden. Das bedeutet, dass der Wert für $k$ sukzessive erhöht wird und „neue“ Routen, deren Homotopieklasse noch nicht gefunden wurden, in die Rückgabemenge aufgenommen werden. Bei einer bereits „bekannten“ Route, deren Homotopieklasse bereits in der Rückgabemenge enthalten ist, wird die kürzeste dieser Routen verwendet.

\subsubsection{Archetypische Distanz}

In diesem Abschnitt wird die archetypische Distanz (siehe Abschnitt 4.4.2) untersucht, also das Verhalten von Routen, die einem konkreten Archetypen zugeordnet sind, zu Routen anderer Archetypen. Es sind zwei Erkenntnisse $\mathrm{zu}$ erwarten. Zum einen sollten Routen, die eine geringe archetypische Distanz zu einem konkreten Archetypen - und somit auch zueinander - besitzen, bestimmte Gemeinsamkeiten im Datenraum aufzeigen. Zum anderen sollten Routen mit einer hohen archetypischen Distanz - und somit einer Zuordnung zu unterschiedlichen Archetypen - gewisse Verschiedenheiten besitzen.

Diese Vermutungen werden im Szenario „Doom“ untersucht. Bezogen auf eine gegebene Eingabemenge von Routen und eine bestimmte Anzahl an Archetypen wird der Begriff Top-Routen wie folgt verwendet: Top-Routen sind genau die Beobachtungen der Eingabemenge, bei denen der $\alpha$-Wert bezüglich eines bestimmten Archetyps größer als ein festgelegter Schwellwert ist. Im Zuge dieser Evaluation wird der Schwellwert auf 0, 8 festgelegt.

Abbildung 4.22 enthält die Top-Routen für vier ermittelte Archetypen im Szenario „Doom“ basierend auf einer Eingabemenge von 400 Routen. Offensichtlich sind diese jeweils zueinander sehr ähnlich - aber nicht identisch -, die Mengen der Top-Routen allerdings weisen jeweils starke Unterschiede auf. Die Top-Routen für Archetyp A1 (Abbildung 4.22a) sind sehr geradlinige und kur- 
ze Routen, die in Variationen die linke Seite des Gebäudeplans durchlaufen. Die dem Archetypen $A 2$ zugeordneten Top-Routen (Abbildung 4.22b) verlaufen ebenfalls im linken Bereich des Gebäudeplans, jedoch sind diese stark gewunden und umlaufen das Hindernis im oberen Teil des Plans ausschließlich linksseitig. Die Top-Routen für Archetyp A3 (Abbildung 4.22c) sind wie auch bei $A 1$ relativ geradlinig und durchlaufen den Gebäudeplan auf der rechten Seite, während die dem Archetyp $A 4$ zugeordneten Routen (Abbildung 4.22d) in Variationen das Zentrum der Karte passieren.

In Abbildung 4.22 ist ebenfalls ersichtlich, dass die Mengen der Top-Routen unterschiedliche Mächtigkeiten besitzen, also unterschiedlich viele Routen beinhalten. Dies unterstreicht die Tatsache, dass unterschiedliche Archetypen eine gegebene Eingabemenge von Beobachtungen unterschiedlich gut repräsentieren.

Anhand von Szenario „Office“ sollen nun die ermittelten Top-Routen (Abbildung 4.23) mit den realisierten archetypischen Routen - also den ausgewählten Repräsentanten der Archetypen - verglichen werden (siehe Abbildung 4.18). Es ist leicht ersichtlich, dass die Mengen der Top-Routen durchaus auch andere Wege durch den Gebäudeplan nehmen als deren Repräsentanten, jedoch gleichzeitig gewisse Gemeinsamkeiten aufrechterhalten. Die realisierte archetypische Route für Archetyp A1 (schwarze Route in Abbildung 4.18) wurde als geradlinig und nahezu in Luftlinie vom Start zum Ziel verlaufend beschrieben. Die entsprechenden Top-Routen (Abbildung 4.23a) unterscheiden sich nun von diesem Repräsentanten derart, dass sie annähernd den gesamten Gebäudeplan bedecken. Die beschriebene Hauptcharakteristik, nämlich die strikte und geradlinige Form, bleibt jedoch stets erkennbar. Die realisierte archetypische Route für $A 2$ (rote Route in Abbildung 4.18) wurde als gewunden und mit vielen Richtungswechseln beschrieben, die stets im unteren Bereich des Gebäudeplans verläuft. Dies gilt bis auf wenige Variationen ebenfalls für die entsprechende Menge der Top-Routen (Abbildung 4.23b). Schließlich wurde die realisierte archetypische Route für $A 3$ (grüne Route in Abbildung 4.18) als eine sehr lange Route mit vielen Windungen und Richtungswechseln beschrieben, die eher im oberen Bereich des Gebäudeplans verläuft. Dies ist erneut in den Top-Routen erkennbar (Abbildung 4.23c), da sie eine Vielfalt an unterschiedlichen Routen darstellen, die vornehmlich relativ lang sind.

Insgesamt bestätigt dies also die Vermutung, dass Routen mit einer geringen archetypischen Distanz Ähnlichkeiten im Datenraum besitzen und sich von Routen mit einer hohen archetypischen Distanz unterscheiden.

\subsubsection{Aggregation von Homotopieklassen}

Schließlich soll untersucht werden, wie sich die archetypische Distanz bezüglich der Aggregation von Homotopieklassen verhält. Dazu wird überprüft, aus wie vielen unterschiedlichen Homotopieklassen sich die Menge der Top-Routen zusammensetzen. Abbildung 4.24 zeigt dazu für das Szenario „White House“ die Eingaberouten und die daraus realisierten archetypischen Routen für un- 


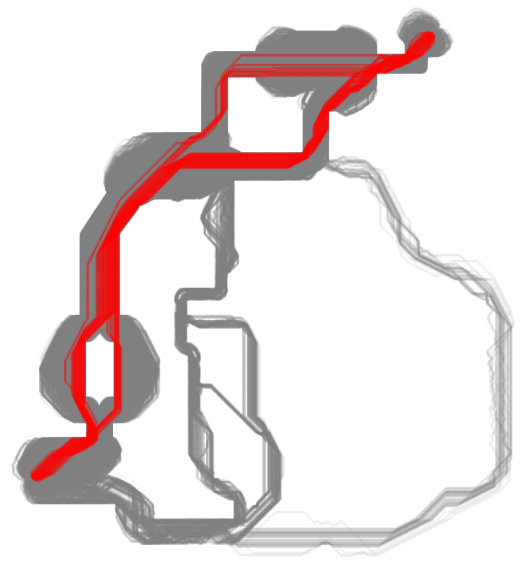

(a) Top-Routen für Archetyp $A 1$.

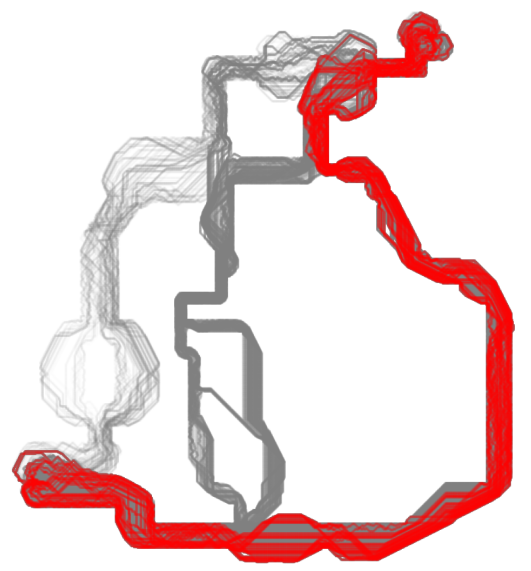

(c) Top-Routen für Archetyp A3.

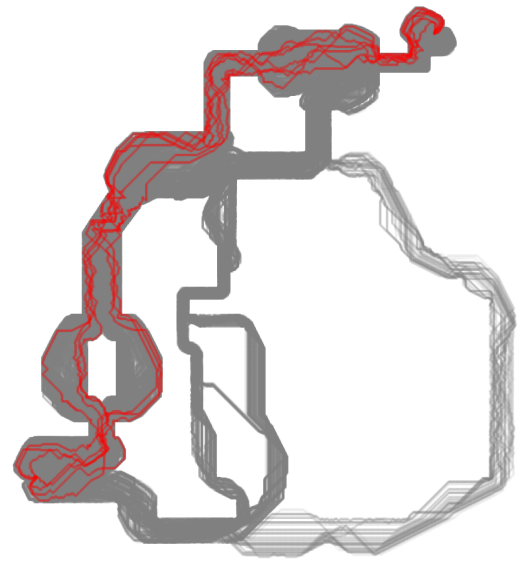

(b) Top-Routen für Archetyp $A 2$.

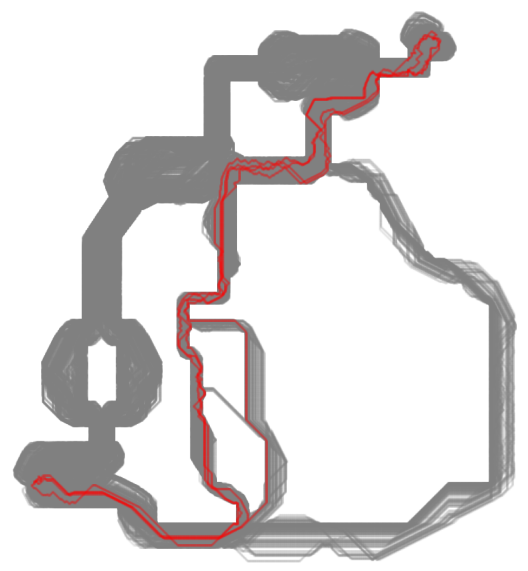

(d) Top-Routen für Archetyp A4.

Abbildung 4.22: Für jeden der vier identifizierten Archetypen im Szenario „Doom“ sind die Top-Routen in rot eingezeichnet. Jede dieser Routen besitzt einen entsprechenden $\alpha$-Wert größer als der Schwellwert von 0,8. Die grauen Routen im Hintergrund stellen die komplette Eingabemenge der 400 Routen dar. Wände und Hindernisse wurden für eine bessere Betrachtung der Abbildung ausgelassen. 


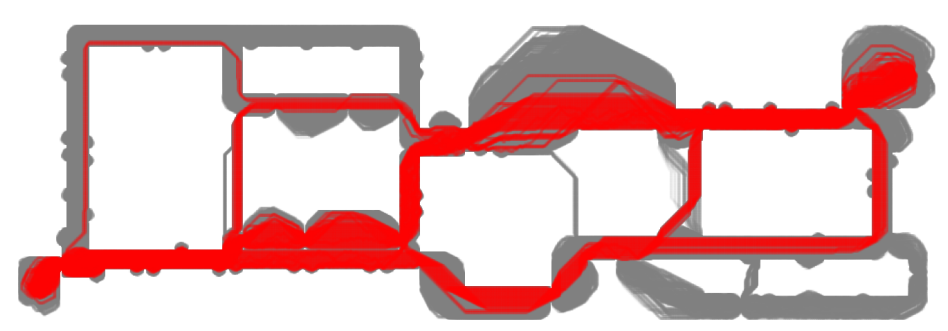

(a) Top-Routen für Archetyp $A 1$.

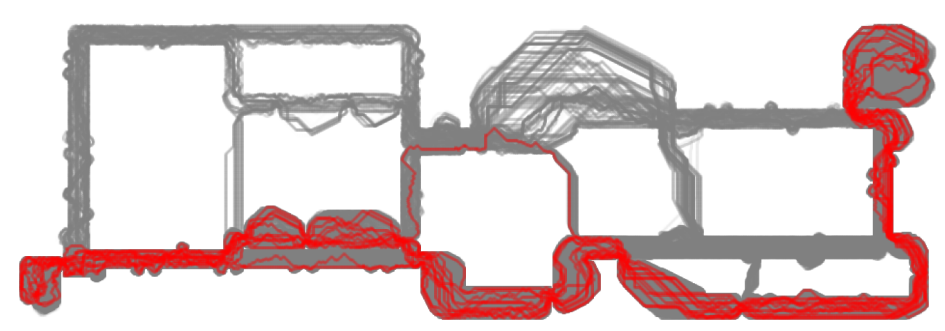

(b) Top-Routen für Archetyp $A 2$.

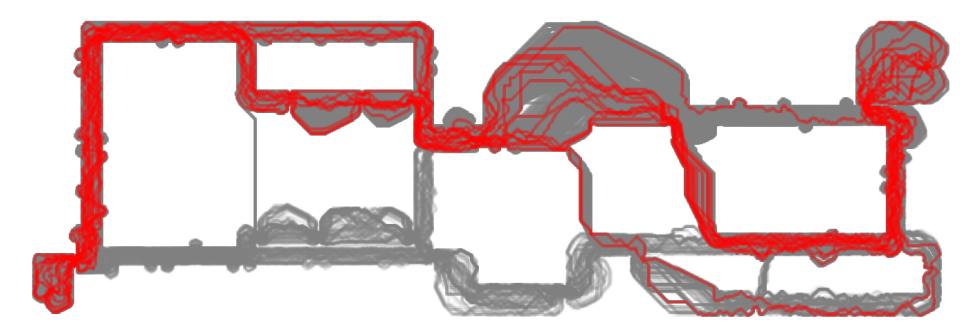

(c) Top-Routen für Archetyp $A 3$.

Abbildung 4.23: Top-Routen mit einem Schwellwert von 0,8 für die drei identifizierten Archetypen im Szenario „Office“. Erneut ist die Eingabemenge grau dargestellt, Wände und Hindernisse sind zur besseren Betrachtung der Hindernisse ausgelassen. 


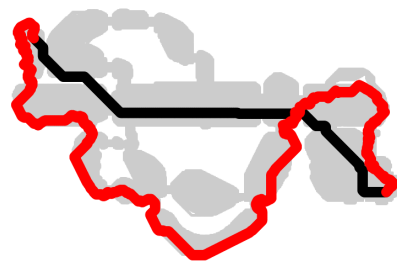

(a) $k=2$

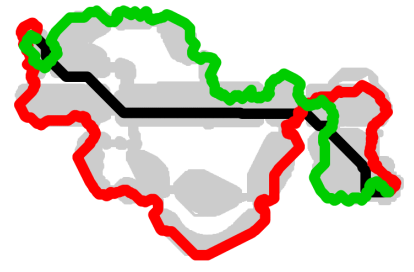

(b) $k=3$

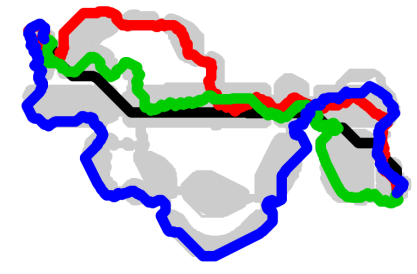

(c) $k=4$

Abbildung 4.24: Realisierte archetypische Routen für unterschiedliche Werte $k$ im Szenario „White House“. Die Eingabemenge ist grau dargestellt, Wände und Hindernisse sind zur besseren Betrachtung der Hindernisse ausgelassen.

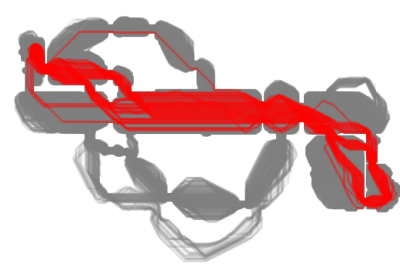

(a) Archetyp A1.

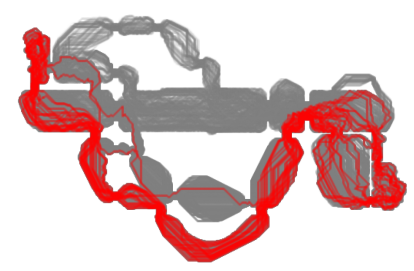

(b) Archetyp A2.

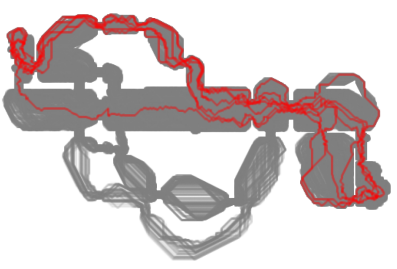

(c) Archetyp $A 3$.

Abbildung 4.25: Top-Routen mit einem Schwellwert von 0,8 für die drei identifizierten Archetypen im Szenario „White House“.

terschiedliche Werte für $k$. In Abbildung 4.25 sind beispielhaft die Top-Routen für $k=3$ mit einem Schwellwert von 0,8 abgebildet.

Wie auch bei dem vorherigen Experiment werden für eine gegebene Eingabemenge von 400 Routen unterschiedlich viele Archetypen berechnet und anschließend Mengen von Top-Routen gebildet, wobei alle enthaltenen Routen einen entsprechenden $\alpha$-Wert größer einem Schwellwert von 0,8 besitzen. Bei einer Wahl von $k=2$ Archetypen wird die Eingabemenge von 400 Routen auf 139 Top-Routen reduziert. 87 der Archetyp A1 zugeordneten Top-Routen verteilen sich auf 10 Homotopieklassen, während 52 Routen aus 20 unterschiedlichen Homotopieklassen dem Archetypen $A 2$ zugeordnet werden. Wenn $k=3$ gewählt wird (siehe Abbildung 4.25), so werden 42 Routen aus 8 unterschiedlichen Homotopieklassen dem Archetypen $A 1$ zugeordnet, 24 Routen aus 7 Homotopieklassen dem Archetypen $A 2$ und 7 Routen aus 4 Homotopieklassen dem Archetypen $A 3$. Schließlich werden bei der Wahl von $k=427$ Routen aus 7 Klassen kombiniert $(A 1), 4$ Routen aus 4 Klassen $(A 2)$, 5 Routen aus 4 Klassen $(A 3)$ und 29 Routen aus 9 Klassen $(A 4)$. Es kann also beobachtet werden, dass die Aggregation von Routen mittels der archetypischen Distanz viele unterschiedliche Homotopieklassen berücksichtigt. 


\subsection{Zusammenfassung}

Grundsätzlich existiert eine überabzählbare Menge von Routen zwischen einem Start- und Zielpunkt. Dies ist der Tatsache geschuldet, dass es unendlich viele Möglichkeiten gibt, zwei Punkte miteinander zu verbinden. Zudem sind diese Möglichkeiten nicht abzählbar. Im vorigen Kapitel wurde durch ein auf Homotopie basierendes Verfahren vorgestellt, mit dem eine abzählbare Menge von Routen zwischen einem Start- und Zielpunkt erstellt werden kann. Diese ist jedoch immer noch eine unendliche Menge, da jede Windung einer Route um ein Hindernis einer neuen Äquivalenzklasse entspricht. Die ebenfalls im vorigen Kapitel vorgestellten Heuristiken zum Finden nicht-homotoper Pfade, der Penalty-Algorithmus und der One-Patching-Algorithmus, erstellen zwar eine endliche Menge von Routen. Wie beschrieben kann diese Menge jedoch abhängig von der untersuchten geografischen Umgebung immer noch sehr groß sein.

In dem vorliegenden Kapitel wurden nun Verfahren vorgestellt, um eine potentiell hohe Anzahl an Routen derart zu filtern, dass eine möglichst kleine Menge von möglichst unterschiedlichen Routen erhalten bleibt. Der Fokus liegt somit auf dem Vergleich geospatialer Trajektorien.

In Abschnitt 4.3 wurde entsprechend ein Algorithmus vorgestellt, der Routen bewertet, um somit eine Rangfolge zu erstellen (siehe die erste Herausforderung in Abschnitt 4.1). Hierzu wird als Bewertungskonzept die Überlastungswahrscheinlichkeit verwendet, was der Annahme folgt, dass wenn ein Punkt häufig auf einem kürzesten Pfad liegt, dieser auch häufig von Personen durchlaufen wird. Es werden also Berechnungen des kürzesten Pfades verwendet anstatt komplexer mikroskopischer Simulationen. Ebenso ist die Integration realer historischer Daten von Personenströmen möglich.

Als zukünftige Arbeit ist die Verwendung der annotierten Gebäudepläne mit den potentiell verkehrsreichen und verkehrsarmen Flächen angedacht, um gegebene Routen durch das Gebäude vergleichen zu können. Die Reihenfolge und die Art der durchlaufenen stark frequentierten Flächen könnten als eine Art Fingerabdruck dienen, womit eine Distanz erstellt werden kann.

In Abschnitt 4.4 wurde die Verwendung der archetypischen Analyse - einer statistischen Methode zur Untersuchung multivariater Datensätze - für Navigationsszenarien in komplexen Umgebungen vorgeschlagen. Insbesondere kann dies als Nachbearbeitungsschritt zur weiteren Analyse von gegebenen (alternativen) Routen dienen. Es wurden abstrakte, realisierte und treue archetypische Routen definiert, deren Fokus nicht nur auf der Geometrie oder Form der Routen liegt, sondern die viele weitere Eigenschaften ausdrücken können. Ferner wurde ein System zur Berechnung archetypischer Routen vorgestellt, mit dem gezeigt wurde, dass eine recht einfache Menge von Merkmalen ausreicht, um gegebene Routen filtern, analysieren und interpretieren zu können. Es werden somit alternative Routen auf extreme Exemplare eingeschränkt, was die zweite Herausforderung dieses Kapitels darstellte. 
Als offene Fragestellung hat sich unter anderem das Synthetisieren von Routen mit bestimmten Eigenschaften herausgestellt, also das gezielte Erstellen sogenannter treuer archetypischer Routen. Die Idee ist dabei, dass ein Anwender mittels „Schieberegler" die gewünschten Ausprägungen der Merkmalsmenge bestimmt und ein Algorithmus eine Beobachtung erstellt, deren Merkmalsausprägungen möglichst exakt denen der Wunschroute entsprechen. Weitere Untersuchungen können bei den zwei vorgestellten Alternativen des Ellbogenkriteriums zur Bestimmung der Anzahl an Archetypen $k$ getätigt werden: die Berücksichtigung der Verteilung der Archetypen auf die Homotopieklassen sowie die Verteilung der Werte der Koeffizientenmatrix $\alpha$. Eine weitere Idee ist der kuratierte Einsatz der Berechnungsergebnisse der archetypischen Analyse als Eingabe von maschinellem Lernen. Ziel dabei wäre das überwachte Lernen von Gesetzmäßigkeiten, die „gute“ alternative Routen ausmachen. Außerdem sollte die Auswirkung unterschiedlicher Eingabemengen untersucht werden. Dies bezieht sich sowohl auf die Vorgehensweise bei der Erstellung der Routen als auch auf die verwendeten Merkmale.

Schließlich wurde ebenfalls in Abschnitt 4.4 ein neuartiges Maß für die Ähnlichkeit von Routen definiert, nämlich die archetypische Distanz (dritte Herausforderung). Dieses Maß erlaubt die Einbeziehung des verwendeten Gebäudeplans und erlaubt die direkte Berechnung der Ähnlichkeit anhand der Merkmalsunterschiede, agiert also im Merkmalsraum und nicht im Datenraum. Mit der archetypischen Distanz können Routen mit Archetypen, Routen mit einem bestehenden Datensatz, aber auch Routen mit zum Datensatz hinzugefügten Beobachtungen verglichen werden. Außerdem stellt die archetypische Distanz ein kontinuierliches Distanzmaß dar, grenzt sich somit von der binär arbeitenden Definition der Homotopie ab und ist in der Lage, Mengen von Routen zu erstellen, die ähnlich aber nicht identisch sind.

Als zukünftige Arbeit ist in diesem Bereich die strukturierte Evaluation eines Systems von Merkmalen geplant, das möglichst einfach zu berechnen ist und in möglichst vielen Anwendungsszenarien verwendet werden kann. Außerdem ist eine detaillierte Analyse der archetypischen Distanz bezüglich der Auswirkung konkreter Merkmale auf die Erstellung der abstrakten Archetypen wünschenswert. 


\section{Identifizierung von Strukturen in Gebäuden}

Im vorigen Kapitel wurden alternative Routen zum einen bewertet und sortiert und zum anderen mittels Clustering gruppiert. In beiden Fällen stand somit die Distanz zwischen beziehungsweise die Ähnlichkeit von Routen im Fokus, es wurde also ein direkter Vergleich durchgeführt. Die wichtigste Erkenntnis des Kapitels war die Tatsache, dass eine starke Wechselwirkung zwischen den Routen und den Karten, in denen sie eingebettet sind, existiert. Im Grunde kann daher auch der Standpunkt eingenommen werden, dass nicht die Routen untersucht werden, sondern die zugrunde liegenden Karten, sodass basierend auf den daraus resultierenden Ergebnissen die Routen ausgewählt werden.

In diesem Kapitel wird nun ein stärkerer Fokus auf die zu untersuchende Karte gelegt, also beispielsweise einen Gebäudeplan. Es werden Ansätze vorgestellt, bei denen der räumliche Eindruck für die Analyse von Routen und Karten miteinbezogen werden kann. Es findet somit entweder direkt oder indirekt eine Identifizierung von Strukturen in Gebäuden statt.

In Abschnitt 5.3 wird dazu das Konzept der diskreten Isovisten eingeführt, um eine Annäherung der lokalen Umgebung einer Route zu erhalten. Als Anwendung dieser Informationen sollen gegebene Routen durch ein Gebäude annotiert werden, beispielsweise an Stellen, an denen eine Tür passiert wird.

Anschließend werden in Abschnitt 5.4 die Konzepte der zwei vorigen Kapitel mit der grundsätzlichen Idee dieses Kapitels kombiniert: es werden archetypische alternative Routen unter der Verwendung von Isovisten-Messgrößen berechnet. Demnach entstehen alternative Routen, die sich nicht mehr auf ihre eigene Geometrie beziehen, sondern ausschließlich auf die durchlaufene lokale Umgebung.

Schließlich werden ebenfalls in Abschnitt 5.4 Isovisten-Messgrößen mittels der archetypischen Analyse untersucht, jedoch nicht basierend auf Routen, sondern auf dem Gebäudeplan selbst. Es werden sogenannte archetypische Regionen ermittelt, welche zur Annotation von Umgebungsplänen verwendet werden können und eine Interpretation derselben ermöglichen.

\subsection{Motivation und Herausforderungen}

Die Art und Weise, wie Menschen ihre Umgebung - also die Form und Struktur von Raum - visuell wahrnehmen, hat einen großen Einfluss auf deren Verhalten. Ein sehr simples Beispiel ist die Wahl eines Tisches in einem leeren 
Restaurant: Gäste werden sich nicht zufällig an einen Tisch setzen, sondern diesen bezüglich des räumlichen Eindrucks wählen [158]. In der umfangreichen Literatur bezüglich der räumlichen Orientierung wird gezeigt, dass effizient zu berechnende Deskriptoren existieren, die in der Lage sind, die für das menschliche Verhalten relevanten Eigenschaften von Raum zu erfassen, siehe beispielsweise [80]. Darüber hinaus wurden auch Verbindungen zwischen der räumlichen Geometrie von Straßen und den darin getätigten Entscheidungen wie beispielsweise Abbiegevorgänge identifiziert [65].

Die soeben beschriebene Denkweise wird in Abschnitt 5.3 aufgenommen, indem das Konzept der Isovisten bei der weiteren Verarbeitung von (alternativen) Routen innerhalb komplexer Umgebungen einbezogen wird. Ein Isovist ist das Volumen des Raumes, das von einem bestimmten Punkt aus eingesehen werden kann [15]. Dieses Konzept wird seit Jahrzehnten umfangreich verwendet, um den menschlichen Eindruck unter anderem von Räumen in Gebäuden zu untersuchen. Da sich die vorliegende Arbeit grundsätzlich auf zweidimensionale Karten fokussiert, ist das weitere Verständnis eines Isovisten die Fläche innerhalb einer Karte, die von einem gegebenen Punkt aus eingesehen werden kann.

Innerhalb des besagten Abschnitts werden Isovisten mittels eines einfachen Raycasting-Ansatzes (Strahlen-basierter Ansatz zur Volumenvisualisierung) angenähert und somit diskrete Isovisten definiert. Diese werden weiterverarbeitet, um eine semantische Bewertung von Routen vorzunehmen. Es werden signifikante Veränderungen der lokalen Umgebung identifiziert, um somit Routen zu annotieren. Grundsätzlich können solche Annotationen verwendet werden, um beispielsweise textuelle Repräsentationen von Routen für hörbare Anweisungen zu erstellen. Außerdem können Karten angereichert werden, indem Türen oder andere Strukturen automatisiert erkannt werden, die im Vorfeld nicht in der Karte verzeichnet waren.

Die eigenständige Analyse von räumlichen Eigenschaften mittels entsprechender Deskriptoren ist weitgehend erforscht. Dennoch ist recht unklar, wie solche Sichtbarkeitseigenschaften sinnvoll in ortsbezogene Anwendungen und Dienste wie etwa Navigationssysteme integriert werden können, um einem Anwender eine sinnvolle Auswahl an Routen zu bieten. Dies motiviert Abschnitt 5.4, in dem Merkmale der lokalen Umgebung in einem umgekehrten Weg als in der Psychologie und Verhaltensforschung üblich - verwendet werden. Es werden zuerst die Unterschiede bezüglich der Wahrnehmung von verschiedenen Orten einer Umgebung berechnet, um diese Ergebnisse zur Erstellung von alternativen Routen zu verwenden. Die Routen werden also gewählt, um einem Anwender Routen entsprechend unterschiedlicher Wahrnehmungseigenschaften bei dem Durchlaufen der Umgebung anzubieten. Die Kernidee ist dabei die Verwendung von extremen (archetypischen) Merkmalen, um frei begehbare Flächen in funktionale Bereiche zu gruppieren. Hierzu wird das bereits behandelte Konzept der archetypischen Analyse mit Messgrößen von Isovisten kombiniert. 
Der erste Teil von Abschnitt 5.4 beschreibt ein Verfahren, das Einblicke in die Struktur eines Gebäudes nur unter Verwendung des Gebäudeplans selbst ermöglicht. Hierzu werden Isovisten-Messgrößen für alle erreichbaren Punkte der Karte berechnet und mittels archetypischer Analyse gruppiert. Es wird das Grundprinzip der archetypischen Analyse ausgenutzt, nämlich die Identifikation der extremsten Beobachtungen sowie die Verwendung mehrerer Merkmale zur gleichen Zeit. Dies resultiert in der Klassifikation von Bereichen in Gebäuden und um Gebäude herum in Klassen wie etwa Eingangsbereich, Gang, Halle oder Straße. Im zweiten Teil des Abschnitts wird die Idee von archetypischen Routen (siehe Abschnitt 4.4) erweitert und der Effekt von IsovistenMessgrößen auf die Gruppierung von Routen, die durch ein Gebäude führen, analysiert. Demzufolge wird eine kleine Menge alternativer Routen zwischen zwei Punkten basierend auf Wahrnehmungseigenschaften identifiziert und nicht auf geometrische Eigenschaften der Routen selbst.

Insgesamt fokussiert sich dieses Kapitel auf die Identifizierung von Strukturen in Gebäuden unter Einbeziehung der lokalen Umgebung. Die folgenden vier Herausforderungen sind Kern dieses Kapitels.

1. Ermittlung einer geeigneten Möglichkeit zur Einbeziehung der lokalen Umgebung im Kontext von komplexen Umgebungen und alternativen Routen.

2. Definition einer einfachen Annäherung der lokalen Umgebung, sodass diese von den bereits beschriebenen Ansätzen verwendet werden können.

3. Erstellung eines effizienten Verfahrens, um mittels der Annotation von Routen einen zusätzlichen Einblick in die Eigenschaften von Routen zu erlangen.

4. Erweiterung der Ausrichtung der Algorithmen von Routen auf die zugrunde liegende Karte, indem archetypische Regionen identifiziert werden.

\subsection{Einbeziehung der lokalen Umgebung mittels Isovisten}

Wie bereits beschrieben, fokussieren sich die im Folgenden vorgestellten Ansätze auf die zugrunde liegenden Karten. Für die Erstellung und Analyse von (alternativen) Routen oder konkreten Positionen innerhalb einer Karte sollen Sichtbarkeitseigenschaften der lokalen Umgebung verwendet werden. Dieser Abschnitt erläutert die entsprechenden Grundlagen.

In Abschnitt 5.2.1 wird der Themenkomplex grundsätzlich eingeführt, indem Möglichkeiten zur quantitativen Analyse der Wahrnehmung von Raum 
beschrieben werden. Es wird der Zusammenhang zwischen räumlicher Wahrnehmung und Architektur beleuchtet und es werden wichtige Werkzeuge zur Analyse räumlicher Anordnungen skizziert.

Anschließend wird in Abschnitt 5.2.2 eines dieser Werkzeuge im Detail beschrieben. Es handelt sich um Isovisten, das heisst ein Konzept, das ursprünglich der Kognitionspsychologie entstammt. Die Idee hinter Isovisten sowie definierten Messgrößen werden ausführlich erläutert, da sie für die spätere Anwendung relevant sind. Es wird also auf die erste der vier Herausforderungen, die in Abschnitt 5.1 definiert wurden, Bezug genommen.

\subsubsection{Quantitative Analyse der Wahrnehmung von Raum}

Seit Ende der 1960er Jahre wurden vermehrt empirische und experimentelle Studien bezüglich der Wahrnehmung architektonischen Raums veröffentlicht. Eine frühe Arbeit ist etwa die Studienreihe von Gärling aus den Jahren 1969 und 1970, in der die individuelle Bewertung von Offenheit und Geschlossenheit von räumlichen Konfigurationen - unter anderem in Bezug auf die physische Größe des Raumes - untersucht wurde [83]-[86]. Ähnlich haben Hayward und Franklin 1974 analysiert, welchen Einfluss eingrenzende Elemente, wie etwa Wände oder Bäume, auf die Wahrnehmung von Offenheit haben [103].

In der Forschung bezüglich der Wahrnehmung von Raum wird oft zwischen Objektwahrnehmung und Umgebungswahrnehmung unterschieden [15]. Grob zusammengefasst wird etwas als Objekt beschrieben, wenn es in sich geschlossen und beweglich ist. Eine Umgebung hingegen wird als offen und unbeweglich beschrieben. Gibson definiert eine (visuelle) Umgebung als eine umgebende Anordnung von Oberflächen, die Struktur in das von ihr reflektierte Licht einbringt [92, S. 221]. Es ist also eine Ansammlung sichtbarer Flächen im Raum.

Räumliche Konfigurationen werden für gewöhnlich mittels einer zweidimensionalen Karte abstrahiert und dargestellt. In einem Gebäudeplan können beispielsweise Wände als schwarze Linien eingezeichnet werden. Darauf basierend existieren zahlreiche Werkzeuge, mit denen architektonischer Raum sowie dessen Beschreibung und Wahrnehmung quantitativ beschrieben werden kann. Diese Werkzeuge werden im Folgenden erläutert.

\subsubsection{Wahrnehmung, Kognition und Architektur}

Menschen nehmen ihre Umwelt mittels unterschiedlicher Sinne wie etwa Sehen, Hören oder Tasten wahr. Von den fünf klassischen Sinnen (Sehen, Hören, Tasten, Schmecken, Riechen) ist das Sehen sicherlich für den grundlegendsten und wichtigsten Teil der Erfahrung eines Menschen verantwortlich [119]. Durch die Aufnahme und Verarbeitung optischer Reize können Interpretationen wie etwa Raumwahrnehmung, Objektextraktion und -erkennung sowie Gesichtswahrnehmung durchgeführt werden [165]. Es existieren zahlreiche Arbeiten, in der Kognitionspsychologen, Verhaltensforscher und Umweltpsychologen untersuchen, wie visuelle Eigenschaften einer Umgebung die subjektiven Gefühle 
und das Verhalten von Menschen beeinflussen.

In diesem Kontext zählt eine Arbeit von Best aus dem Jahre 1970 [19] zu den früheren Forschungen, die die Wegfindung und Navigation in komplexen Gebäuden behandelt. In dem Artikel wird analysiert, welche Herausforderungen bei der Wegfindung zu einem Gefühl der Verlorenheit führen können. Es werden neben der Länge der Route und der Anzahl der Richtungswechsel insbesondere die Anzahl der Wahlmöglichkeiten für Teilrouten untersucht. Gleichermaßen führen Hölscher et al. in [108] aus, dass Schwierigkeiten bei der Wegfindung in komplexen Gebäuden auf zwei Aspekten beruhen: einerseits auf dem individuellen räumlich-kognitiven Vermögen des Menschen und andererseits auf der Architektur des Gebäudes. Dies unterstreicht die These, dass die Forschung der architektonischen Gestaltung mit der Forschung der räumlichen Kognition von Menschen verknüpft werden muss, um neue Erkenntnisse im Bereich der Wegfindung innerhalb von Gebäuden zu erlangen. Weitere verwandte Arbeiten innerhalb der Raum- und Umgebungspsychologie behandeln Studien in typischen Gebäuden wie etwa Krankenhäusern [98], Einkaufszentren [57] oder Flughäfen [155].

\subsubsection{Werkzeuge zur Analyse räumlicher Anordnungen}

Wie eingangs erwähnt, existieren unterschiedliche Theorien und Werkzeuge, um räumliche Anordnungen - das heißt die lokale Umgebung - zu analysieren [43]. Einer der grundlegendsten Begriffe in diesem Umfeld ist Space Syntax [107]. Hierbei geht es hauptsächlich um die Erfassung topologischer Strukturen einer Umgebung ohne explizite geometrische Messungen im Euklidischen Raum. Eine Erweiterung dieses Begriffs ist durch Visibility Graph Analysis (VGA) [175] gegeben, wobei insbesondere die Sichtverbindungen zwischen Orten fokussiert und daraus ein Sichtbarkeitsgraph erstellt und untersucht wird.

Ein weiteres fundamentales Konzept, was ebenfalls als Space Syntax verwendet wird, lautet Isovist und wurde bereits 1967 von Tandy eingeführt [171]. Ein Isovist beschreibt die Menge aller Punkte im Raum, die von einem spezifischen Punkt aus sichtbar sind. Im Jahre 1979 hat Benedikt in [15] eine formale Definition von Isovisten zusammen mit einem Satz an analytischen Messgrößen vorgestellt, womit eine quantitative Beschreibung von räumlichen Umgebungen ermöglicht wird. Ebenfalls in [15] wurden Isovisten-Felder als die Darstellung einer kompletten Umgebung mittels Isovisten-Messgrößen beschrieben. Durch eine diskretisierte Repräsentation der Umgebung - sei es mithilfe speziell ausgewählter oder gleichmäßig verteilter Orte - können Isovisten-Felder angenähert werden [12], [148]. Für die Diskretisierung der Umgebung gibt es jedoch keine allgemeingültigen Empfehlungen bezüglich der Wahl repräsentativer Orte oder der Granularität der Diskretisierung. Es gilt stets einen Kompromiss zwischen Abdeckung der Umgebung und Kosten für die Berechnung zu finden [41]. In den meisten zu dem Thema existierenden Forschungsarbeiten wird die Analyse von Isovisten auf 2D-Repräsentationen wie einfache Karten oder Gebäudepläne angewandt. Es gibt jedoch auch Literatur, in der die Analyse von 
Isovisten im 3D-Raum untersucht wird [64].

In zahlreichen Studien haben Isovisten-Messgrößen eine starke Prognosefähigkeit für räumliches Verhalten bewiesen. So haben Wiener und Franz in [181] die Beziehungen zwischen Isovisten-Messgrößen und der menschlichen Lösung navigationsbezogener Aufgaben festgestellt. Die Probanden sollten unter anderem den Ort für den besten Überblick und den Ort für das beste Versteck finden. Davies et al. haben in [40] die Verwendung von Isovisten-Messgrößen untersucht, um Vorhersagemodelle für die räumliche Orientierung von Menschen zu erstellen. Die Erkenntnisse werden dazu genutzt, automatisiert Orte zu identifizieren, an denen eine Orientierung potentiell schwierig ist. Diese können wiederum dazu verwendet werden, entsprechende Hinweise sowohl in der Karte als auch in der Umgebung aufzunehmen. Weiterführende Literatur ist in [32], [160], [182] zu finden. Darin wird mittels unterschiedlicher Fallstudien auch innerhalb von Gebäuden hervorgehoben, dass Isovisten-Messgrößen wichtige Informationsquellen für die individuelle Entscheidungsfindung bei der Wegwahl darstellen.

\subsubsection{Analyse von Isovisten}

Eine der wichtigsten Fragen der Verhaltensforschung und Umweltpsychologie ist die nach der Verbindung zwischen objektiven visuellen Eigenschaften einer Umgebung und der entsprechenden subjektiven Reaktion eines Menschen. Wie im vorigen Abschnitt bereits erläutert, wurde die erste Verwendung des Begriffs Isovist von Tandy getätigt, indem er einen Isovisten als die Menge von Punkten in einem Raum, die von einem gegebenen Punkt aus gesehen werden kann, definiert [171]. Daher können Isovisten als eindeutige „Fingerabdrücke“ einer spezifischen räumlichen Konfiguration an einem bestimmten Punkt angesehen werden. Dieser Fingerabdruck ist nicht zwingend eindeutig in einer Umgebung, jedoch haben identische Isovisten identische Eigenschaften. Basierend auf der Arbeit von Tandy hat Benedikt eine formale Definition von Isovisten entwickelt und eine Menge von Messgrößen eingeführt, die die numerische Untersuchung einer räumlichen Umgebung ermöglicht [15]. Somit sind die Eigenschaften der „Fingerabdrücke“ quantitativ zu erfassen.

Die formalisierte Definition von Isovisten ist laut [15] wie folgt (siehe auch Abbildung 5.1): Gegeben ein Euklidischer dreidimensionaler Raum $E^{3}$ und eine einfach zusammenhängende Fläche $D$ mit ihrer Grenzlinie $\partial D$. Nun werden zusammenhängende, materiell und vom Menschen sichtbare Punkte als ,reale Oberfläche“ $S$ definiert. Eine Umgebung $E$ besteht aus der Ansammlung $S$ von realen Oberflächen und deren konkreter räumlicher Anordnung innerhalb der Fläche $D$. Jede Änderung der Menge oder der Anordnung von $S$ lässt eine neue Umgebung $E^{\prime}$ entstehen. Umgangssprachlich ausgedrückt ist $E^{3}$ der uneingeschränkte dreidimensionale Raum und $D$ der zu analysierende Teil des Raums. Die Grenzlinie $\partial D$ kann man sich als die Begrenzung einer Karte vorstellen und $S$ als die Mauern eines Gebäudes oder andere Hindernisse. 


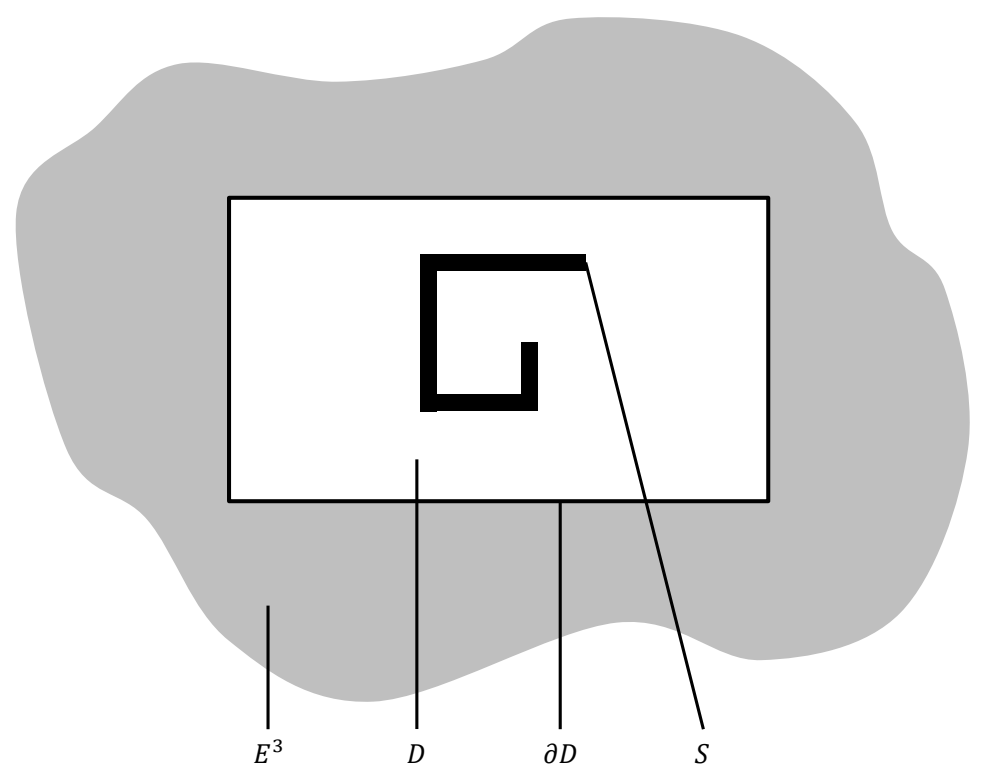

Abbildung 5.1: Grundbegriffe im Umfeld von Isovisten. Abbildung angelehnt an [15], Figure 1 und 2.

Für jeden Punkt $x$ in $D$ ist die Menge

$$
V_{x}=\{v \in D: v \text { ist sichtbar von } x\}
$$

der Isovist am Standpunkt $x$. Anders ausgedrückt ist $V_{x}$ die Menge von Punkten in $D$, die vom konkreten Standpunkt $x$ aus sichtbar ist.

Ein Isovist ist eine punktierte Menge $(V, x)$, sodass $V_{x}=V$. Der Standpunkt ist demnach per Definition Teil des Isovisten. Es ist möglich, dass zwei unterschiedliche Isovisten die gleiche Untermenge $V$ von $D$ besitzen, was bedeutet, dass die sichtbaren Punkte identisch sind, jedoch der Standpunkt nicht. Dies kann beispielsweise in einem kreisförmigen Raum der Fall sein.

Nun kann die Grenzlinie $\partial V_{x}$ des Isovisten in drei Teile aufgeteilt werden:

1. Reale Oberflächen $S_{x}$, die durch Wände und Hindernisse gegeben sind (rot dargestellt in Abbildung 5.2).

2. Verdeckte radiale Grenzlinien $R_{x}$, also gedachte Sichtlinien, die an den Rändern von Hindernissen beginnen und an Wänden oder aber der Grenzlinie $\partial D$ der zusammenhängenden Fläche $D$ enden (blaue Linien in Abbildung 5.2).

3. Oberflächen $\partial D_{x}$ der zusammenhängenden Fläche $D$ (grüne Linie in Abbildung 5.2), die gewissermaßen das „Ende“ des eingezeichneten Bereiches einer Karte darstellt. 


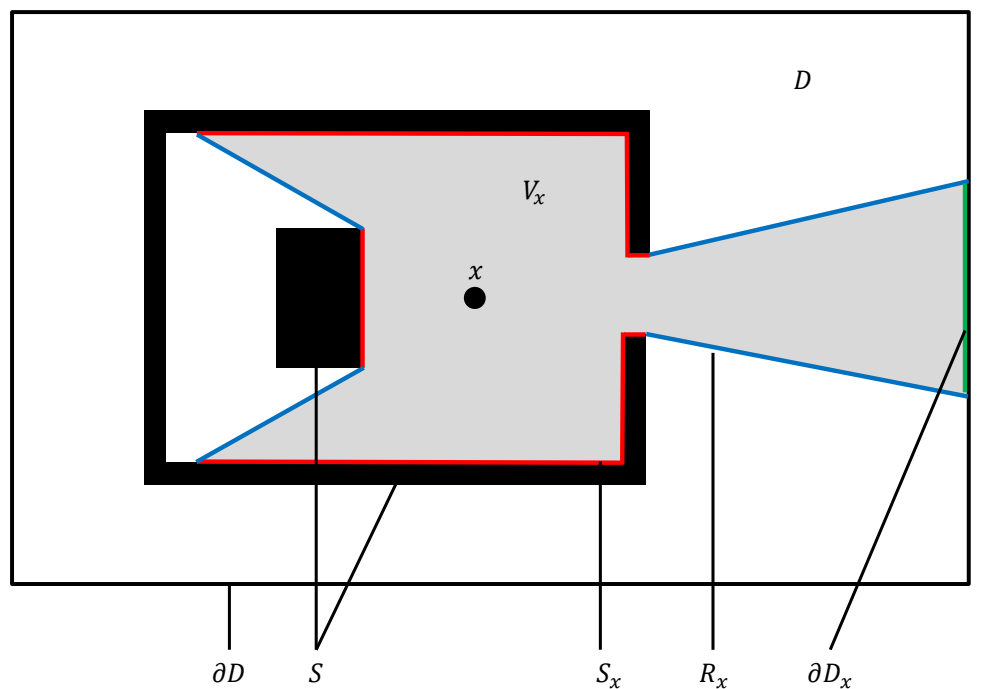

Abbildung 5.2: Veranschaulichung der unterschiedlichen Isovisten-Grenzlinien.

Isovisten können sowohl in 3D als auch in 2D berechnet werden. Aufgrund der Tatsache, dass sich die vorliegende Arbeit auf 2D-Gebäudepläne konzentriert, wird $V_{x}$ als eine horizontale 2D-Ebene durch den Punkt $x$ im 3D-Raum angesehen.

Benedikt schlägt in [15] eine zweite Definition für Isovisten vor, die eine Alternative für Gleichung 5.1 darstellt. Demnach kann ein Isovist auch als Menge von Strecken angesehen werden, die den Standpunkt $x$ mit den Punkten $v^{\prime}$ auf der sichtbaren Grenzlinie $\partial V_{x}-R_{x}$ des Isovisten verbinden. Es folgt:

$$
V_{x}=\left\{\left[x, v^{\prime}\right]: v^{\prime} \in\left(\partial V_{x}-R_{x}\right)\right\} .
$$

Diese Strecken werden im weiteren Verlauf auch als Strahlen bezeichnet, da sie am Standpunkt $x$ beginnen und von dort sternförmig in alle Richtungen ausstrahlen. Die Strahlen besitzen eine Länge

$$
l_{x, \theta}=d\left(x, v^{\prime}\right)=\left\|v^{\prime}-x\right\|=\sqrt{\left(v_{1}^{\prime 2}-x_{1}^{2}\right)+\left(v_{2}^{\prime 2}-x_{2}^{2}\right)},
$$

wobei $x_{1}, x_{2}$ die Koordinaten des Startpunkts $x$ sind, $v_{1}^{\prime}, v_{2}^{\prime}$ die Koordinaten des Endpunkts $v^{\prime}$ und $0 \leq \theta \leq 2 \pi$ die Ausrichtung repräsentiert. Wenn die Länge der Strahlen $l_{x, \theta}$ als Funktion aufgefasst wird, so ist $L_{x}(\theta)$ die entsprechende Verteilungsfunktion.

Basierend auf der Definition von Isovisten mittels Strahlen (Gleichung 5.2) kann nun eine Menge statistischer Messgrößen $m\left(V_{x}\right)$ definiert werden. Diese werden im Folgenden erläutert:

area Die Messgröße area ist definiert durch $A_{x}=A\left(V_{x}\right)$ und beschreibt den Flächeninhalt des Isovisten. In Abbildung 5.2 ist dies die grau dargestellte 


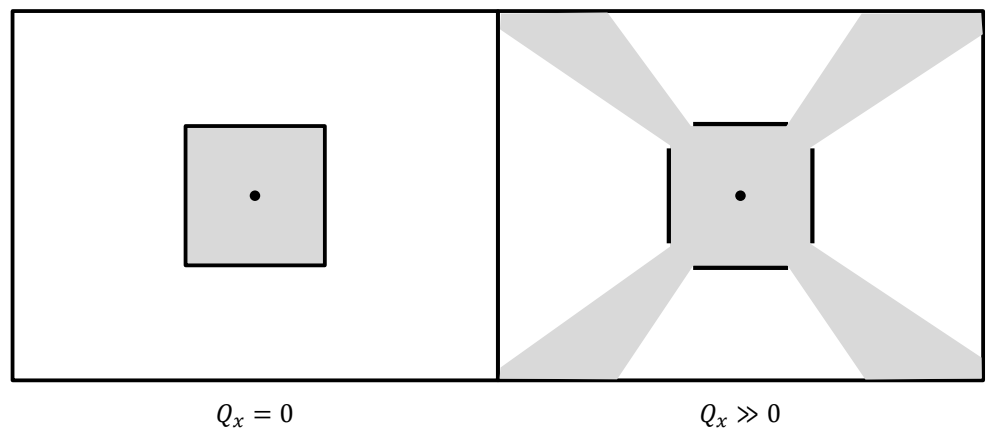

Abbildung 5.3: Veranschaulichung der Isovisten-Messgröße occlusivity $Q_{x}$. Abbildung angelehnt an [15], Figure 8.

Fläche, die vom Standpunkt $x$ aus eingesehen werden kann (entspricht dem eigentlichen Isovisten). Je größer der Wert ist, desto mehr Raum kann vom gegebenen Standpunkt aus überblickt werden. Gleichzeitig bedeutet dies, dass der Standpunkt von einer großen Fläche aus beobachtet werden kann.

real-surface perimeter Die Messgröße real-surface perimeter $P_{x}=\left|S_{x}\right|$ beschreibt die Umfangslänge der sichtbaren Hindernisoberfläche wie beispielsweise Wände. In Abbildung 5.2 ist dies die Länge der vom Isovisten „berührten“ Kanten der Wände und des Hindernisses (rot eingezeichnet).

occlusivity Die Messgröße occlusivity $Q_{x}=\left|R_{x}\right|$ beschreibt die Länge der verdeckten radialen Grenzlinie. In Abbildung 5.2 ist dies die Länge der Sichtlinien, die an den Rändern der Hindernisse beginnen und an den Wänden oder der Kartenbegrenzung enden (blau eingezeichnet). Dieser Wert bezieht sich auf die Tiefe der Verdeckung von Hindernissen und deutet mit einem Wert größer Null die Möglichkeit einer Unsicherheit bei der visuellen Wahrnehmung an. Je näher ein Hindernis am Standpunkt liegt, desto mehr Sicht wird dem Betrachter verdeckt und desto höher ist dieser Wert. Es besteht zudem eine Wechselwirkung zwischen occlusivity und real-surface perimeter, da ein nahes Hindernis und entsprechend hohe occlusivity viel von den sonst sichtbaren Wänden verdeckt (weniger real-surface perimeter).

Abbildung 5.3 zeigt ein Beispiel mit $Q_{x}=0$ und somit keiner Verdeckung (linkes Szenario) sowie ein Beispiel mit $Q_{x} \gg 0$ und somit starker Verdeckung und hoher Unsicherheit (rechtes Szenario).

variance Ein Isovist definiert die Menge von Punkten in einem Raum, die von einem gegebenen Punkt aus gesehen werden können. Mit Gleichung 5.2 wurde die Definition eines Isovisten in eine Menge von Strahlen abgeändert. Die Messgröße variance $M_{2, x}=M_{2}\left(l_{x}, \theta\right)$ ist das zweite zentrale Moment bezüglich 
der Längen der Strahlen und beschreibt die Abweichung vom arithmetischen Mittel der Längen.

skewness Während variance das zweite zentrale Moment ist, stellt die skewness $M_{3, x}=M_{3}\left(l_{x}, \theta\right)$ nach einer Normierung das dritte zentrale Moment dar. Skewness bezieht sich ebenfalls auf die Längen der Strahlen (das heißt die Abstände des Standpunkts zu den sichtbaren Punkten in der Umgebung) und beschreibt die Abweichung von einer symmetrischen Verteilung.

circularity Sowohl variance als auch skewness sind Werte, die mit der Kompaktheit assoziiert sind, einer intrinsischen Eigenschaft von zweidimensionalen Formen [15]. Im Grunde ist die Kompaktheit ein numerischer Wert, der beschreibt, wie ähnlich die Form einem Kreis ist. Sie wird in zahlreichen Anwendungsfällen wie etwa der Mustererkennung oder dem maschinellen Sehen angewandt [138]. Circularity bezieht sich ebenfalls auf die Kompaktheit und insbesondere auf die isoperimetrische Ungleichung [146]. Diese schätzt für zweidimensionale Formen den Flächeninhalt gegen den Umfang ab. Circularity ist demnach ein isoperimetrischer Quotient, der mittels $N_{x}=\left|\partial V_{x}\right|^{2} / 4 \pi A_{x}$ berechnet wird, wobei $\left|\partial V_{x}\right|$ wie beschrieben die Umfangslänge des Isovisten darstellt. Da ein Kreis bei gegebenem Umfang den größten Flächeninhalt einschließst, ist dieser Wert größer gleich 1.

Die genannten Messgrößen sind rotationsinvariant gegenüber dem Standpunkt $x$ und laut [15] nur eine mögliche Auswahl, die ergänzt oder verändert werden kann. Für die Berechnung der Messgrößen variance und skewness, die sich beide auf die Länge der Strahlen beziehen, wird in [15] eine große, aber finite Anzahl an Strahlen verwendet, sodass die erhaltenen numerischen Werte eine Annäherung an die wahren Werte darstellen.

Der „Charakter" eines Isovisten $V_{x}$ - repräsentiert durch dessen Form und Größe - ist wie beschrieben spezifisch bezüglich des Standpunkts $x$ und der gegebenen Umgebung $E$. Die Isovisten zweier benachbarter Standpunkte sind unterschiedlich, jedoch ähnlich. Dies kann laut [15] derart interpretiert werden, dass diese Veränderung der Isovisten mit der Empfindung eines Beobachters korreliert, der sich auf einem Pfad $\Pi$ in $D-E$ bewegt. Die Sicht und Sichtbarkeit des Beobachters kann sich bei der Bewegung graduell, aber auch sprunghaft verändern. Daher wird in [15] vorgeschlagen, dass für ein kumulatives Verständnis der Umgebung auch die Transformation der vom Beobachter bei der Bewegung empfangenen Informationen analysiert wird.

Formell ausgedrückt bedeutet dies, dass jeder Punkt $x$ im Raum $D-S$, der möglicherweise Teil eines Pfades $\Pi$ ist, durch die numerischen Werte des Messgrößen-Vektors $\left[A_{x}, P_{x}, Q_{x}, M_{2, x}, M_{3, x}, N_{x}\right]$ charakterisiert ist. Wie beschrieben ändern sich die Messgrößen von Standpunkt zu Standpunkt, weshalb die numerischen Werte variierend im Raum sind und demnach Skalarfelder darstellen. Diese werden im folgenden Isovisten-Felder genannt und stellen eine alternative Beschreibung einer Umgebung dar. 


\subsection{Diskrete Isovisten als Approximation der lokalen Umgebung}

Im vorigen Abschnitt wurde eine Möglichkeit vorgestellt, die lokale Umgebung im Kontext von komplexen Umgebungen sowie alternativen Routen zu beschreiben. Ziel dieses Abschnittes ist nun die Vorbereitung einer ersten Anwendung dieser räumlichen Informationen. Dazu werden im weiteren Verlauf sogenannte Isovisten verwendet, um eine effiziente Annotation von Routen mittels deren lokaler Umgebung zu erhalten. In Abschnitt 5.2.2 wurde das Konzept der Isovisten und der entsprechenden Messgrößen ausführlich erläutert. Es ist zu erkennen, dass je nach Gebäudegeometrie eine potentiell hohe Berechnungskomplexität besteht (siehe auch [75]). Wenn ein detailliertes GISModell der Umgebung verwendet wird, so existieren bei der Berechnung von Isovisten komplizierte Fallunterscheidungen und nicht-triviale GIS-Anfragen wie etwa das Finden des nächstgelegenen geschnittenen Liniensegments, das Überprüfen, ob ein Dreieck leer ist, oder das Aufzählen aller Segmente, die in ein Dreieck fallen. Es bestehen zwar Methoden zur effizienteren Bearbeitung der Anfragen wie etwa die räumliche Indexierung mittels $R^{*}$-Bäumen [14], jedoch bleibt die generelle Komplexität hoch. Außerdem ist die Berechnung von Isovisten in einem GIS-Modell stark abhängig von der zugrunde liegenden Geometrie. Lange Liniensegmente können die Leistung von Beschleunigungen wie etwa der Indexierung vermindern. Das Unterteilen der Geometrie in kleinere Liniensegmente, die kurz genug für effiziente Anfragen sind, erhöht jedoch die Anzahl der zu betrachtenden Segmente und somit die Komplexität der Ausführung.

Die Idee ist nun eine effiziente Annäherung von Isovisten durch die Definition diskreter Isovisten. Der Fokus liegt hierbei auf der Einfachheit des Ansatzes und akzeptiert durchaus einen Fehler. Auch an dieser Stelle muss ein Kompromiss zwischen Genauigkeit und Ausdruckskraft gefunden werden. Die Vorgehensweise bei der Erstellung diskreter Isovisten und entsprechender Messgrößen ist folgende: mittels eines einfachen Raycasting-Verfahrens werden radial um den Bezugspunkt Strahlen ausgesandt, um somit die Messgrößen anzunähern. Diese werden anschließend dazu verwendet, signifikante Veränderungen der lokalen Umgebung zu messen und somit Routen oder auch Karten zu annotieren. Dies kann einer semantischen Bewertung selbiger dienen.

Zusammengefasst bezieht sich dieser Abschnitt auf zwei der vier Herausforderungen, die in Abschnitt 5.1 beschrieben wurden: zum einen findet eine einfache Annäherung der lokalen Umgebung statt und zum anderen eine effiziente Annotation von Routen.

\subsubsection{Definition und Berechnung diskreter Isovisten}

Im weiteren Verlauf dieser Arbeit wird das ursprüngliche Konzept eines Isovisten nach der in [15] vorgestellten Definition exakter Isovist genannt, auch 
wenn dies in Bezug auf die originalen Messgrößen nicht vollständig korrekt ist. In einer zweidimensionalen Umgebung ist der von einem Standpunkt einzusehende Raum, also der Isovist, eine einfach zusammenhängende Fläche. Die Messgrößen area, real-surface perimeter, occlusivity und circularity können wie in Abschnitt 5.2.2 beschrieben - direkt berechnet werden. Einzig die Messgrößen variance und skewness beziehen sich auf die Definition eines Isovisten als Menge von Strahlen und stellen eine Annäherung dar: je mehr Strahlen verwendet werden, desto genauer sind die beiden Messgrößen. Dennoch kann die eigentliche Definition von Isovisten nach [15] als Begriff der exakten Isovisten angesehen werden.

Die Definition diskreter Isovisten als Approximation exakter Isovisten folgt der Idee, effizienter zu einer Lösung zu gelangen, indem ein gewisser Diskretisierungsfehler akzeptiert wird. Im ersten Schritt wird der gegebene Gebäudeplan bezüglich einer konstanten Länge gerastert und die Geometrie somit in Pixel überführt. Hierdurch können Überschneidungen von Liniensegmenten mittels Algorithmen zur Rasterung von Linien wie etwa dem Bresenham-Algorithmus [22] berechnet werden. Dies bildet die Grundlage für die schnelle Annäherung exakter Isovisten. Im Grunde verfolgt der Ansatz der diskreten Isovisten die alternative Definition von Isovisten als Menge von Strahlen (siehe Gleichung 5.2). Diese Vorgehensweise ist laut [15] notwendig, um die Messgrößen variance und skewness zu berechnen. Die Idee bei einem diskreten Isovisten ist, dass dieser und dessen Messgrößen ausschließlich über die Strahlen berechnet werden. Die Anzahl der zu erstellenden Strahlen muss jedoch sorgfältig gewählt werden. Falls die Anzahl zu hoch ist, können die Berechnungskosten sehr hoch werden; wenn die Anzahl zu klein ist, besteht die Möglichkeit, dass wichtige Eigenschaften oder auch Teile der Gebäudegeometrie übersehen werden. Ebenso verhält es sich mit der Wahl des Diskretisierungsparameters, also der Größe der Pixel. Alle Messgrößen, die von diskreten Isovisten abgeleitet werden, sind beschränkt durch die Genauigkeit der Repräsentation der Umgebung durch Pixel.

Gegeben sei ein Belegungsraster in Form einer monochromen Bitmap, das die zu analysierende Fläche $D$ darstellt. Weiße Pixel repräsentieren frei begehbare Flächen und schwarze Pixel entsprechend Hindernisse wie Wände oder Möbel. Teile der schwarzen Pixel korrespondieren demnach mit der Ansammlung realer Oberflächen $S$ aus der Terminologie von [15].

Zur Annäherung eines Isovisten wird nun ein Raycasting-Verfahren verwendet. Es wird ein Parameter $\theta$ definiert, dann werden entsprechend $360 / \theta$ gleichmäßig verteilte Strahlen radial vom gegebenen Standpunkt ausgesendet. Die Ausbreitung eines Strahls stoppt, wenn die Oberfläche eines Hindernisses oder die Begrenzung der Karte erreicht ist. Für jeden Strahl des Standpunkts werden der Parameter $\theta$, die Koordinaten des Start- und Endpunkts sowie dessen Länge gespeichert.

Neben der Anzahl der auszusendenden Strahlen existiert der Parameter $\gamma$, der als Schwellwert zur Begrenzung der maximalen Länge eines Strahls verwen- 

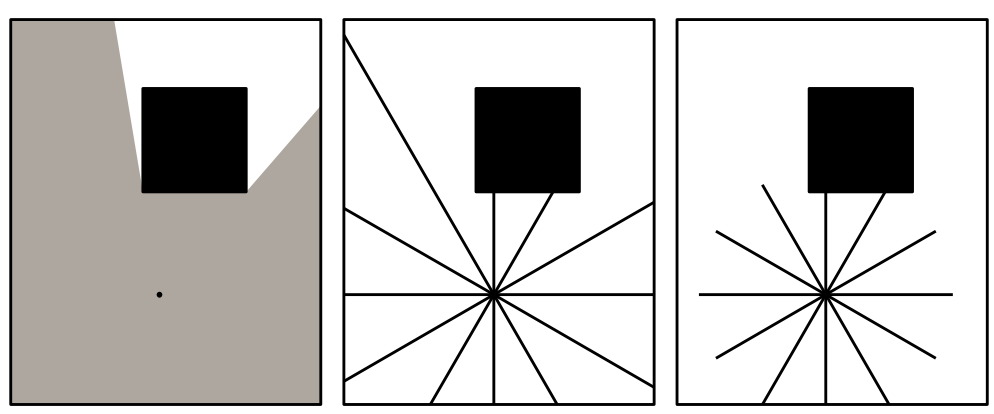

Abbildung 5.4: Ein beispielhafter exakter Isovist, ein diskreter Isovist mit $\theta=$ 30 und $\gamma=\infty$ sowie ein diskreter Isovist mit $\theta=30$ und $\gamma=30$ (von links nach rechts).

det wird. Die Idee hinter diesem Parameter ist erneut der Fokus auf Effizienz, durchaus unter der Akzeptanz eines Fehlers. Wenn der Parameter $\gamma$ auf unendlich gesetzt wird, stoppt die Ausbreitung der Strahlen nur beim Schneiden eines Hindernisses oder der Kartenbegrenzung. Ist $\gamma$ mit einer natürlichen Zahl belegt, so stoppt die Ausbreitung des Strahls spätestens beim Erreichen der besagten Länge. Die Auswahl der Parameterbelegung hat Auswirkungen sowohl auf die Laufzeit des Algorithmus als auch auf die resultierenden IsovistenMessgrößen. Abbildung 5.4 zeigt einen exakten Isovisten, einen diskreten Isovisten mit $\theta=30$ und $\gamma=\infty$ sowie einen diskreten Isovisten mit $\theta=30$ und $\gamma=30$ (von links nach rechts).

Das eigentliche Ziel der Annäherung exakter Isovisten durch diskrete Isovisten ist die Ermittlung der Messgrößen und nicht der Isovist selbst. Die in der Vorveröffentlichung [75] vorgeschlagenen Definitionen für die Messgrößen von diskreten Isovisten lauten wie folgt:

area Die Messgröße area $\left(A_{x}\right)$ eines diskreten Isovisten ist definiert durch den Flächeninhalt des Polygons, das durch das paarweise Verbinden der Endpunkte der Strahlen entsteht. Hierzu können das Polygon trianguliert und die Flächeninhalte der Dreiecke addiert oder aber die Gaußsche Trapezformel verwendet werden. Der direkte Zusammenhang zum Parameter $\theta$ ist offensichtlich, da eine größere Menge von Strahlen eine präzisere Annäherung des Polygons bedeutet.

variance und skewness Die Messgrößen variance $\left(M_{2, x}\right)$ und skewness $\left(M_{3, x}\right)$ werden über das zweite beziehungsweise dritte zentrale Moment bezüglich der Längen der Strahlen berechnet, wobei die Länge der Strahlen auf der Euklidischen Norm basiert. Die Messgrößen stellen demnach die Varianz und Schiefe der Länge der Strahlen dar und sind insbesondere vom Parameter $\gamma$, der maximalen Strahlenlänge, abhängig. 
circularity Die Messgröße circularity ist wie bei der ursprünglichen Definition durch $N_{x}=\left|\partial V_{x}\right|^{2} / 4 \pi A_{x}$ definiert. Im Zähler steht die quadrierte Umfangslänge des Isovisten, was bei den diskreten Isovisten die Umfangslänge des Polygons ist, das durch die paarweise Verbindung der Endpunkte der Strahlen entsteht. Im Nenner ist der Flächeninhalt $A_{x}$ aufgeführt, der bereits beschrieben wurde.

real-surface perimeter und occlusivity Durch den Raycasting-Ansatz können die Messgrößen real-surface perimeter $\left(P_{x}\right)$ und occlusivity $\left(Q_{x}\right)$ nicht angemessen angenähert werden. Der Grund dafür ist die Tatsache, dass die ausgesendeten Strahlen nicht zwingend die Endpunkte der Gebäudegeometrie schneiden, weshalb die Verdeckung nicht richtig wiedergegeben werden kann.

Bei der Berechnung der vier möglichen Messgrößen gilt es zu bedenken, dass die Diskretisierung der Umgebung mittels Rasterkarten systematische Fehler einführt. Ein Beispiel bezieht sich auf die Entscheidung, ab wann ein Pixel schwarz statt weiß dargestellt wird. Bei einer Rasterung ist es für gewöhnlich so, dass ein Pixel, das auch nur eine geringfügige Menge von Gebäudegeometrie beinhaltet, als belegt angesehen und somit schwarz dargestellt wird. Wenn bei Messgrößen die Anzahl an Pixeln verwendet wird, so sollte berücksichtigt werden, dass alle numerischen Aussagen bezüglich Freiflächen (weiße Pixel) kleiner als in der Realität sein werden, da schwarze Pixel nicht zwingend voll durch Gebäudegeometrie gefüllt sein müssen.

Zusammengefasst kann gesagt werden, dass die soeben beschriebene Definition der diskreten Isovisten-Messgrößen nur Annäherungen der Messgrößen der exakten Isovisten sind. Um die nicht abgedeckten Messgrößen real-surface perimeter und occlusivity trotzdem annähern zu können, müsste zusätzlich ein Shadow Casting Verfahren eingesetzt werden.

\subsubsection{Evaluation}

In diesem Abschnitt sollen die soeben definierten diskreten Isovisten sowie deren Messwerte evaluiert werden. Hierzu wird ein Szenario einer komplexen Umgebung verwendet, um zum einen die Möglichkeit zur Annotation von Routen zu untersuchen. Zum anderen werden die Messgrößen der diskreten Isovisten mit denen exakter Isovisten verglichen. Die Betrachtung der Korrelation soll die Eignung der Approximation verdeutlichen.

\subsubsection{Annotation von Routen}

Eine erste mögliche Anwendung der diskreten Isovisten-Messgrößen ist die Untersuchung von Pfaden, die durch einen Gebäudeplan laufen. Die Idee dabei ist, signifikante Veränderungen der lokalen Umgebung zu identifizieren, um somit Routen zu annotieren. Wenn darüber hinaus eine geeignet große Menge von Routen durch einen Gebäudeplan geführt und analysiert wird, so können die Annotationen der Routen auf den Gebäudeplan übertragen werden. Es findet 
sozusagen eine Annotation eines Gebäudeplans auf Basis seiner „Verwendung“ statt.

Im Folgenden wird eine Route als Vektor von durchlaufenen $x$ - und $y$ Koordinaten dargestellt. Entsprechend der Koordinaten werden die verschiedenen Isovisten-Messgrößen berechnet, um vier Zeitreihen $A_{x}, M_{2, x}, M_{3, x}$ und $N_{x}$ zu erhalten. Diese werden jeweils bezüglich der lokalen Minima und Maxima untersucht, wofür ein Parameter $\mu$ für die Größe der lokalen Umgebung (im Sinne des ,Sliding-Window“-Ansatzes, etwa Schiebefensteransatzes) benötigt wird. In der bereits in [75] vorgestellten Implementierung werden die resultierenden lokalen Extrema sowohl im Merkmalsraum (Zeitreihendarstellung) als auch im Datenraum (Pfad im Gebäudeplan) markiert. In der Zeitreihendarstellung sind die lokalen Minima als vertikale, grüne Linien dargestellt und die lokalen Maxima als vertikale, rote Linien. Im Gebäudeplan sind die lokalen Minima entsprechend als grüne Kreise eingezeichnet und die lokalen Maxima als rote Quadrate.

Für die nachfolgende Evaluation wird der Gebäudeplan des Hauptgebäudes der Technischen Universität München (TUM) verwendet. Es wurden zahlreiche Analysen durchgeführt. Nachfolgend wird ein beispielhaftes und repräsentatives Ergebnis in Form einer Route, die das Gebäude in einem großen Kreis durchläuft, dargestellt. Die Route beginnt am Haupteingang des Gebäudes, der sich an der rechten Seite im Gebäudeplan befindet und verläuft im Uhrzeigersinn.

In diesem Abschnitt werden für jeden Punkt der Route sowohl die diskreten als auch die korrespondierenden exakten Isovisten-Messgrößen berechnet. Anschließend werden die lokalen Minima und Maxima sowohl in der Zeitreihendarstellung als auch im Gebäudeplan hervorgehoben. Wie bereits angesprochen, repräsentiert der Parameter $\mu$ die Größe der lokalen Umgebung zur Berechnung der lokalen Extrema. Dieser Parameter wurde für den gegebenen Gebäudeplan empirisch ermittelt und mit $\mu=300$ fixiert. Für die Anzahl der auszusendenden Strahlen wurde $\theta=1$ gesetzt. Es werden also pro Punkt der Route 360 radiale Strahlen berechnet. Die Skalen wurden bei der Zeitreihendarstellung bewusst ausgelassen, da bei der Untersuchung der lokalen Minima und Maxima lediglich die signifikanten Unterschiede der numerisch stark unterschiedlichen Messgrößen betrachtet werden. Die Interpretation bezieht sich stets auf die diskreten Extrema, die exakten Werte sind zum Vergleich zusätzlich dargestellt.

Die Ergebnisse bezüglich des Flächeninhalts $A_{x}$ (area) sind in Abbildung 5.5 visualisiert. Das lokale Maximum mit dem höchsten Wert (Maximum ganz rechts in Abbildung 5.5a) ist an der Stelle zu finden, an der die Route den Innenhof durchläuft: die Sicht ist am weitesten, unmittelbar bevor die Route wieder das Gebäude betritt. Ein weiterer recht interessanter Ausschnitt des Werteverlaufs ist der konstante Bereich zwischen dem dritten und fünften Maximum (siehe Abbildung 5.5a etwa in der Mitte), was in Abbildung 5.5b dem langen, vertikalen Flur im linken Bereich des Gebäudes entspricht. Die Mono- 


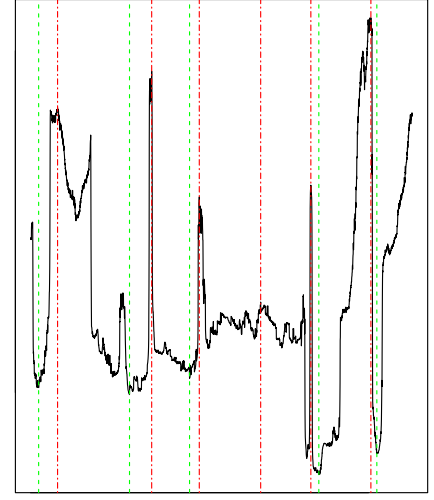

(a) Diskrete Messgrößen in Zeitreihendarstellung.

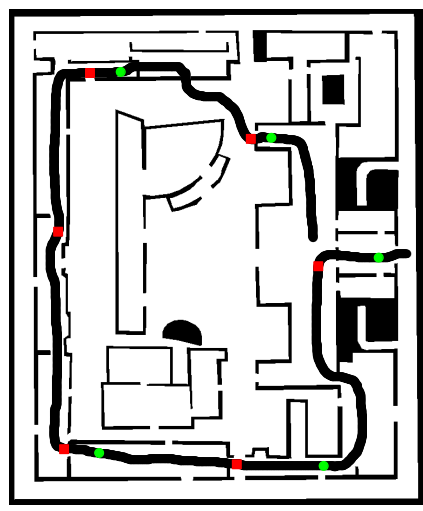

(b) Lokale Extrema der diskreten Messgrößen.

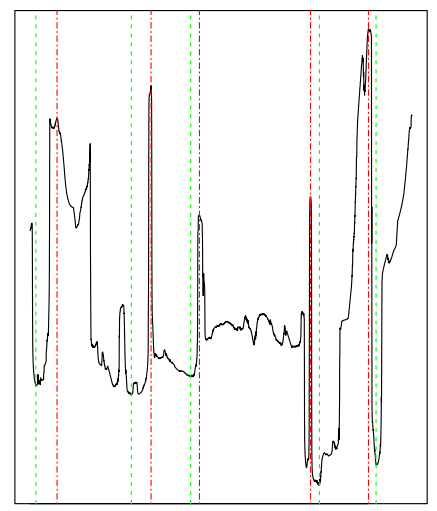

(c) Exakte Messgrößen in Zeitreihendarstellung.

Abbildung 5.5: Lokale Extrema für die Isovisten-Messgröße area $\left(A_{x}\right)$ mit den Parametern $\theta=1, \gamma=\infty$ und $\mu=300$.

tonie des Flures ist damit durch den konstanten Verlauf der Merkmalswerte repräsentiert. Die kleinsten lokalen Minima (die letzten zwei in Abbildung 5.5a) sind die Stellen der Route, an denen ein Fußgänger eingeengt werden würde.

Die Zeitreihen der resultierenden Varianz (variance, $M_{2, x}$ ) der diskreten und exakten Isovisten sowie die Visualisierung der lokalen Extrema sind in Abbildung 5.6 dargestellt. Hervorzuheben ist insbesondere die Tatsache, dass die Maxima exakt an Stellen im Gebäudeplan vorzufinden sind, an denen entweder eine Tür durchlaufen oder an ihr vorbeigegangen wird. Ebenso ist zu erkennen, dass sich die lokalen Minima etwa in den Mitten von Räumen befinden (siehe erstes, zweites und drittes Minimum in Abbildungen 5.6a und $5.6 \mathrm{~b})$.

Die Messgröße skewness $\left(M_{3, x}\right)$, also die Schiefe der Verteilung der Strahlenlängen, wird zumeist im Zusammenhang mit weiteren Variablen verwendet. Wie in [15, S. 59] beschrieben, existieren Situationen, in denen eine Person viel Fläche einsehen möchte, ohne jedoch übermäßiger Beobachtung von anderen ausgesetzt zu sein. In solchen Fällen reicht die alleinige Betrachtung von area nicht aus, stattdessen muss zusätzlich skewness einbezogen werden. Skewness beschreibt im Grunde, ob sich die Strahlen unterschiedlicher Länge in einer bestimmten Region konzentrieren. Ein hoher Wert von area mit gleichzeitig hohem Wert von skewness deutet daher für gewöhnlich Begebenheiten an, in denen ein guter Ausblick mit wenig Exponiertheit herrscht. Dies ist auch im vorliegenden Szenario zu beobachten: das letzte Maximum in Abbildung 5.7a liegt in etwa an der Stelle, an der die Messgröße area ihr höchstes lokales Maximum (siehe Abbildung 5.5) besitzt, nur ein wenig früher. An dieser Stelle können offensichtlich große Teile des Innenhofs sowie der obere Bereich der Karte eingesehen werden, ohne jedoch vom gesamten Innenhof beobachtbar zu sein. Die lokalen Minima hingegen sind an Stellen vorzufinden, an denen die Sicht eine gewisse Symmetrie besitzt. Dies ist im Gebäudeplan an den 


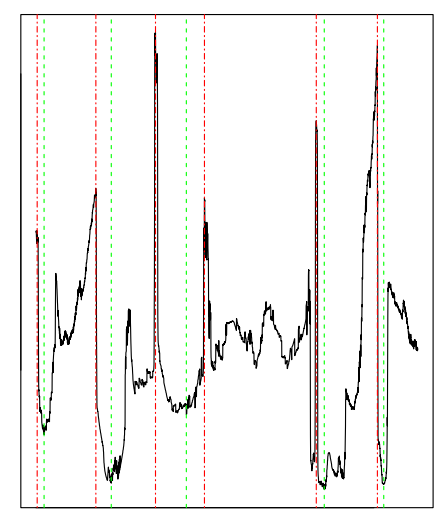

(a) Diskrete Messgrößen in Zeitreihendarstellung.

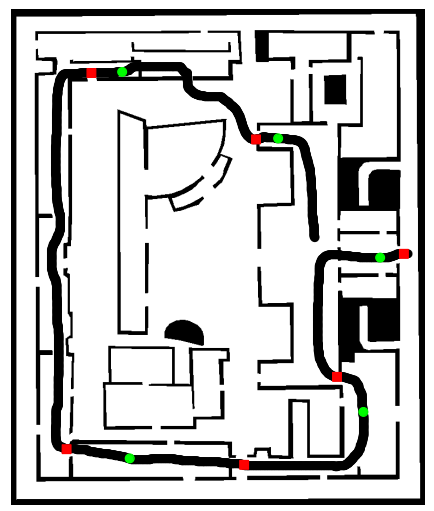

(b) Lokale Extrema der diskreten Messgrößen.

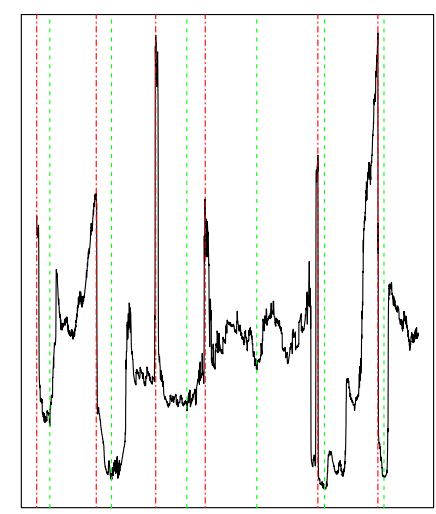

(c) Exakte Messgrößen in Zeitreihendarstellung.

Abbildung 5.6: Lokale Extrema für die Isovisten-Messgröße variance $\left(M_{2, x}\right)$ mit den Parametern $\theta=1, \gamma=\infty$ und $\mu=300$.

drei durchlaufenen Türen gegeben sowie im linken Bereich der Karte bei den parallel passierten Eingängen (siehe Abbildung 5.7b).

Schließlich sind in Abbildung 5.8 die Ergebnisse der Messgröße circularity $\left(N_{x}\right)$ dargestellt. Diese haben sich allerdings zur Annotation von Routen als wenig nützlich herausgestellt.

Zusammengefasst kann gesagt werden, dass die diskreten IsovistenMessgrößen als Approximation zur Einbeziehung der lokalen Umgebung verwendet werden können. Im Folgenden wird der Zusammenhang zwischen den angenäherten und den exakten Messgrößen näher untersucht. Die Vermutung ist, dass die Verwendung weniger Strahlen (höherer Wert von $\theta$ ) eine geringere Genauigkeit verursacht. Ebenso werden die Auswirkungen untersucht, die die Begrenzung der maximalen Strahlenlänge (Parameter $\gamma$ ) verursachen.

\subsubsection{Korrelation der Messgrößen}

In diesem Abschnitt soll die Differenz zwischen den berechneten Messgrößen der diskreten und der exakten Isovisten, also der durch die Diskretisierung entstehende Fehler, untersucht werden. Anders als in der Vorveröffentlichung [75] bezieht sich dieser Abschnitt nicht auf den Annäherungsfehler, der mittels der normalisierten Wurzel der mittleren Fehlerquadratsumme (normalized rootmean-square deviation, NRMSD) beschrieben wurde. Im Grunde sind bei der Betrachtung der signifikanten Unterschiede der unterschiedlich skalierten Messwerte nicht die einzelnen Fehler interessant, sondern die zusammenhängenden Veränderungen beider Verläufe. Daher wird im Folgenden die Korrelation als Vergleichskriterium verwendet.

Im ersten Schritt sollen die berechneten Isovisten-Messgrößen visuell untersucht werden. In Abbildung 5.9 sind die jeweiligen Messgrößen, die entlang der durchlaufenen Route berechnet wurden, eingezeichnet. $\mathrm{Zu}$ sehen sind die 


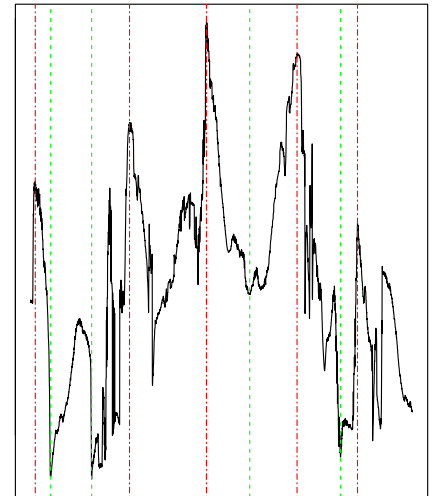

(a) Diskrete Messgrößen in Zeitreihendarstellung.

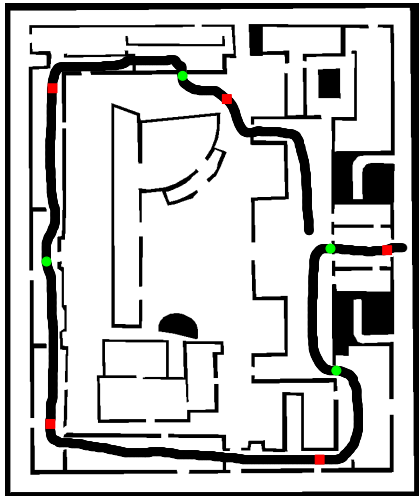

(b) Lokale Extrema der diskreten Messgrößen.

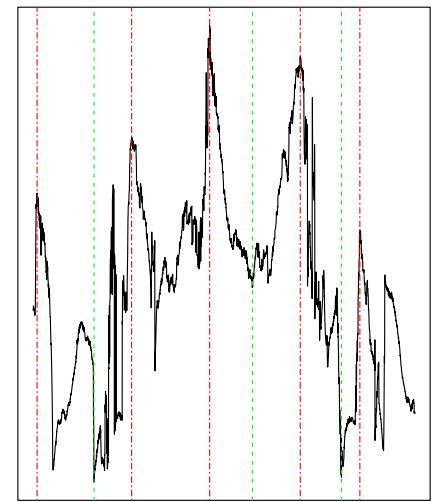

(c) Exakte Messgrößen in Zeitreihendarstellung.

Abbildung 5.7: Lokale Extrema für die Isovisten-Messgröße skewness $\left(M_{3, x}\right)$ mit den Parametern $\theta=1, \gamma=\infty$ und $\mu=300$.

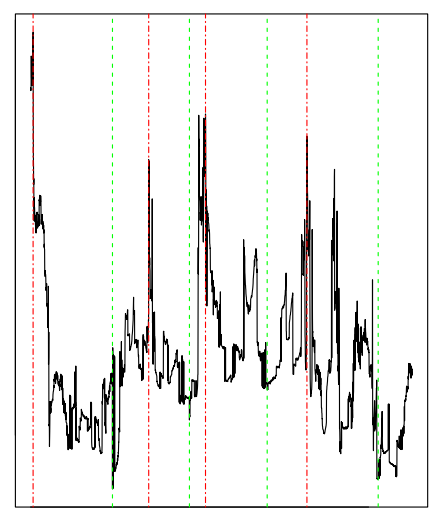

(a) Diskrete Messgrößen in Zeitreihendarstellung.

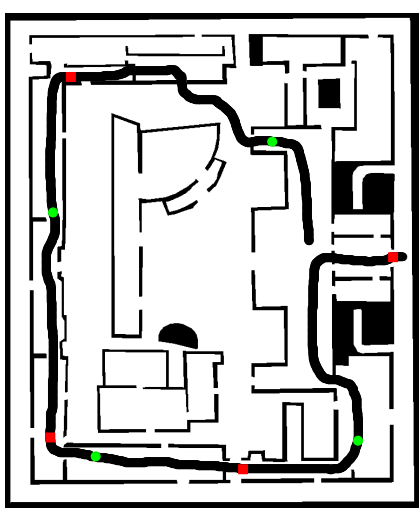

(b) Lokale Extrema der diskreten Messgrößen.

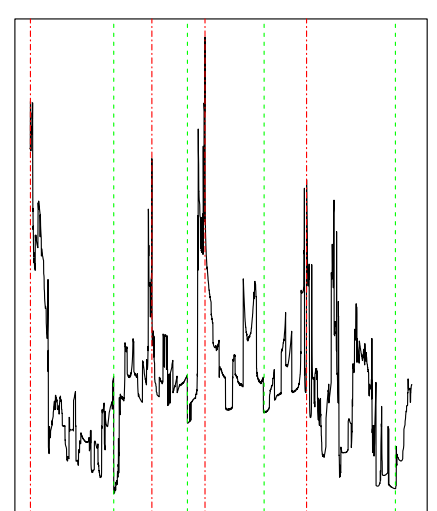

(c) Exakte Messgrößen in Zeitreihendarstellung.

Abbildung 5.8: Lokale Extrema für die Isovisten-Messgröße circularity $\left(N_{x}\right)$ mit den Parametern $\theta=1, \gamma=\infty$ und $\mu=300$. 


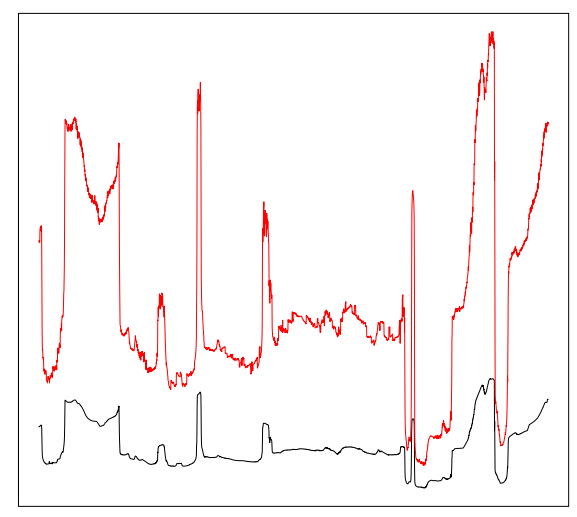

(a) area $\left(A_{x}\right)$

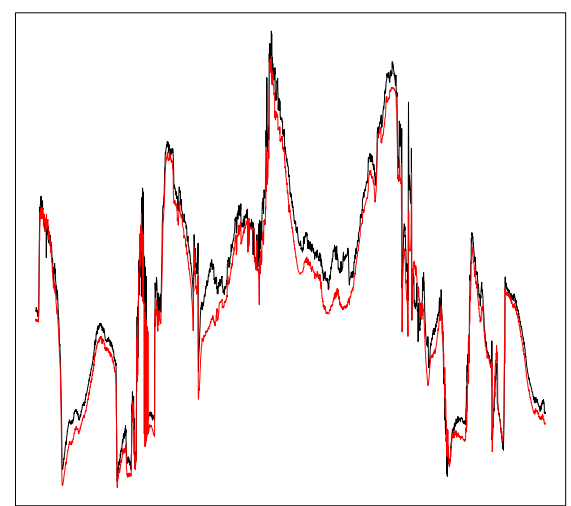

(c) skewness $\left(M_{3, x}\right)$

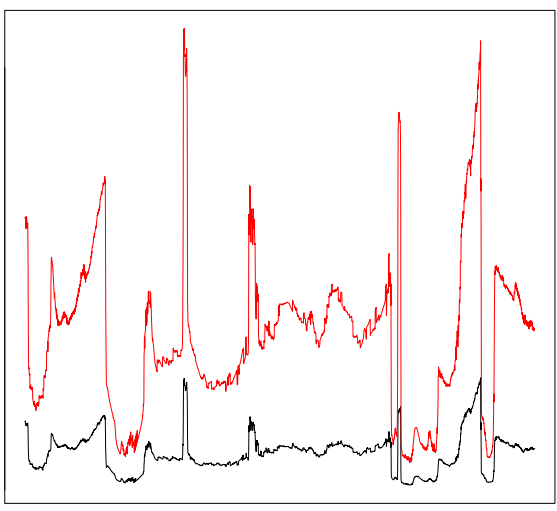

(b) variance $\left(M_{2, x}\right)$

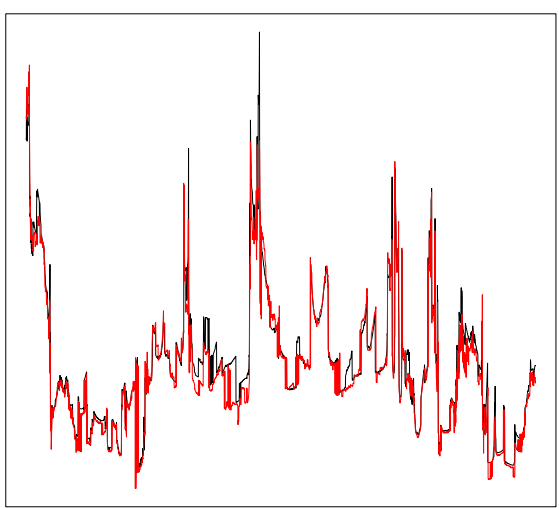

(d) circularity $\left(N_{x}\right)$

Abbildung 5.9: Vergleich der exakten Isovisten-Messgrößen entlang der gegebenen Route in schwarz mit den diskreten IsovistenMessgrößen in rot $(\gamma=\infty, \theta=1)$.

diskreten Messgrößen mit den Parametern $\gamma=\infty$ (keine Beschränkung der maximalen Länge der Strahlen) und $\theta=1$ (360 Strahlen) als rote Zeitreihe. Entsprechend stellen die schwarzen Zeitreihen die Werte der exakten Isovisten dar. Aufgrund der stark unterschiedlichen Skalen sind die Achsenbeschriftungen erneut ausgelassen. Es ist zu erkennen, dass die grundsätzliche Struktur der Werteverläufe der exakten Isovisten-Messgrößen von den diskreten Isovisten beibehalten werden. Aufgrund der Diskretisierung sind die Werteverläufe der diskreten Isovisten-Messgrößen jedoch weniger kontinuierlich.

Abbildung 5.10 zeigt dieselben Ergebnisse der Berechnung, jedoch nach einer Skalierung auf den Wertebereich von 0 bis 1 . Visuell ist der Zusammenhang zwischen den exakten und den diskreten Isovisten-Messgrößen noch deutlicher zu erkennen.

Für den in den Abbildungen 5.9 und 5.10 dargestellten direkten Vergleich der Messergebnisse wurde der maximale Radius der ausgesendeten Strahlen nicht begrenzt, das heißt $\gamma=\infty$ angenommen. Um eine Beschleunigung der Berechnung unter Akzeptanz eines gewissen Fehlers zu erhalten, kann dieser 


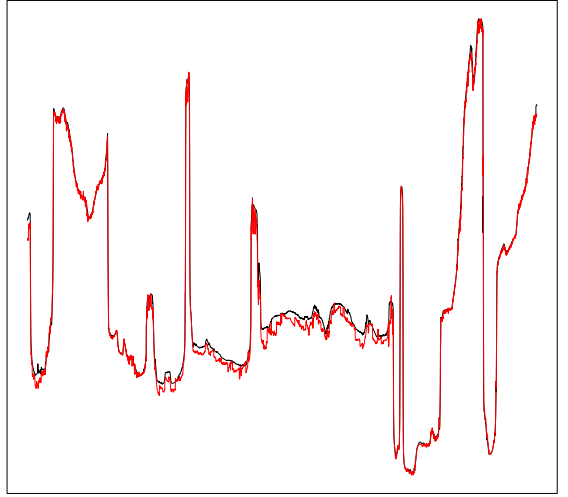

(a) area $\left(A_{x}\right)$

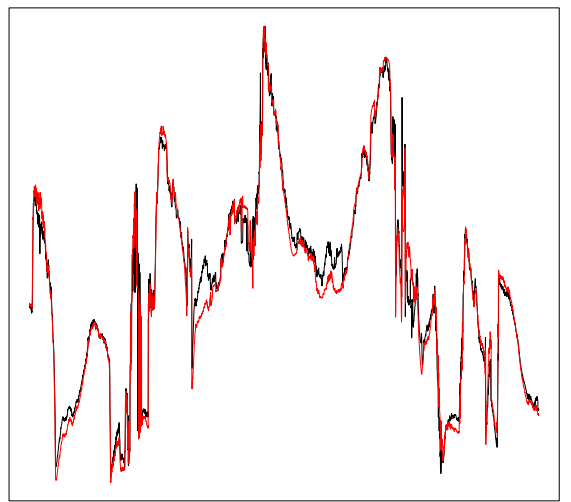

(c) skewness $\left(M_{3, x}\right)$

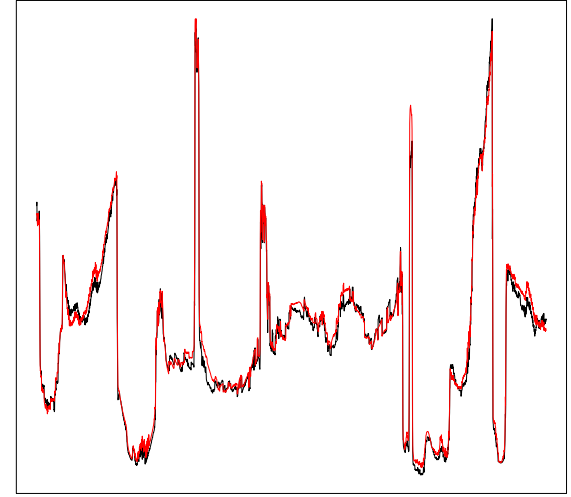

(b) variance $\left(M_{2, x}\right)$

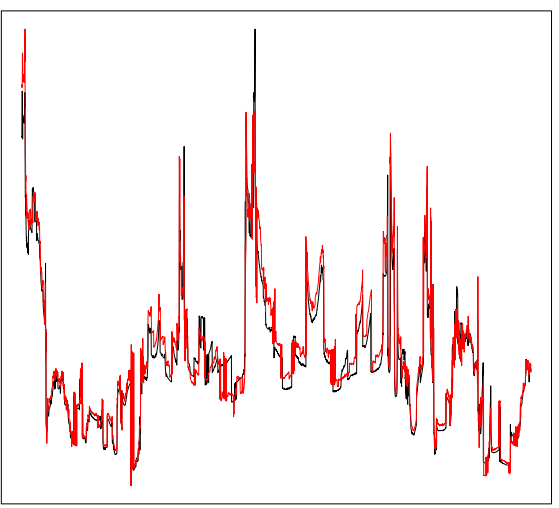

(d) circularity $\left(N_{x}\right)$

Abbildung 5.10: Vergleich der auf das Intervall [0,1] skalierten exakten Isovisten-Messgrößen entlang der gegebenen Route in schwarz mit den ebenso skalierten diskreten Isovisten-Messgrößen in $\operatorname{rot}(\gamma=\infty, \theta=1)$. 


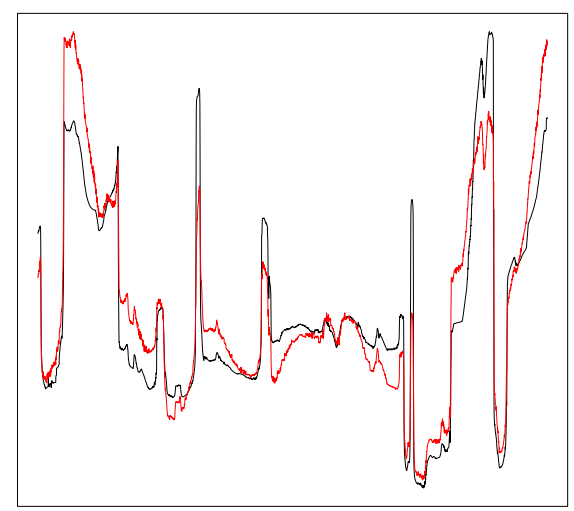

(a) area $\left(A_{x}\right)$

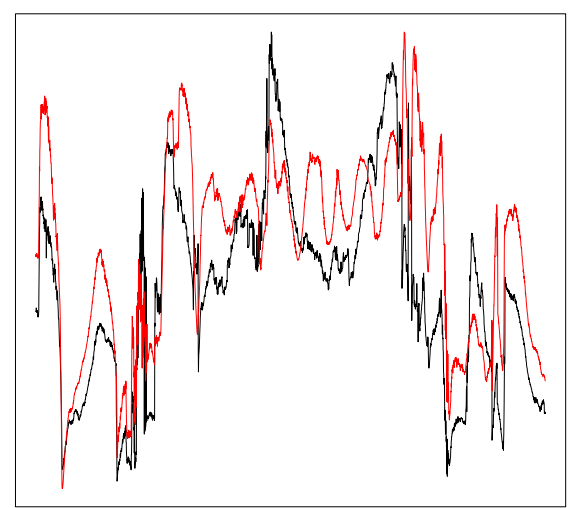

(c) skewness $\left(M_{3, x}\right)$

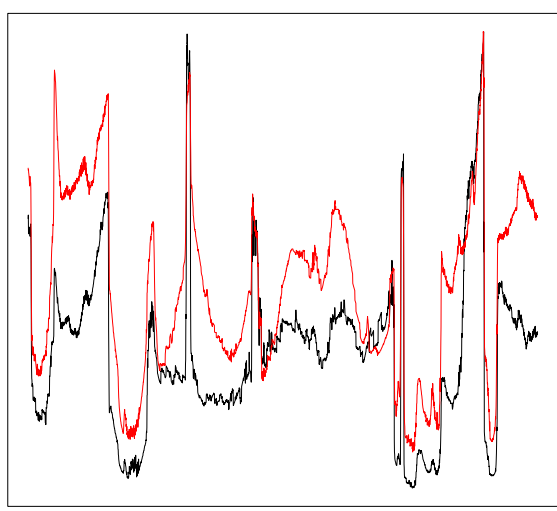

(b) variance $\left(M_{2, x}\right)$

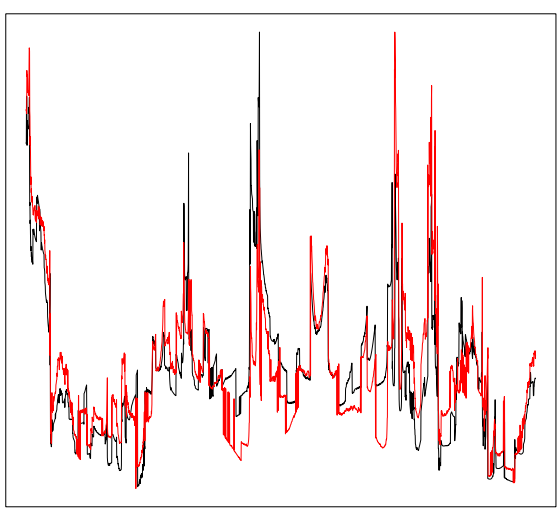

(d) circularity $\left(N_{x}\right)$

Abbildung 5.11: Vergleich der auf das Intervall [0,1] skalierten exakten Isovisten-Messgrößen entlang der gegebenen Route in schwarz mit den ebenso skalierten diskreten Isovisten-Messgrößen in $\operatorname{rot}(\gamma=300, \theta=1)$.

Wert angepasst werden. Diese Anpassung wurde für den gegebenen Gebäudeplan beispielhaft vorgenommen, indem die maximale Strahlenlänge auf 300 Pixel gesetzt wurde $(\gamma=300)$. Die skalierten Ergebnisse sind in Abbildung 5.11 visualisiert. Grundsätzlich ist zu erkennen, dass die Werteverläufe korrelieren, jedoch verschieden starke Ausschläge zu erkennen sind.

Ein weiterer Parameter, der verändert werden kann, ist $\theta$, also die Anzahl der auszusendenden Strahlen. Die Vermutung ist, dass je mehr Strahlen ausgesendet werden, desto näher liegen die Messwerte beieinander. Bevor die Korrelationen der Messergebnisse quantitativ analysiert wird, soll Abbildung 5.12 diesen Effekt visuell verdeutlichen. Beispielhaft ist die diskrete Messgröße area $\left(A_{x}\right)$ dargestellt. Die Werte sind skaliert, die maximale Länge ist nicht beschränkt $(\gamma=\infty)$ und die Anzahl der Strahlen liegt bei $360(\theta=1), 72$ $(\theta=5)$ und $36(\theta=10)$.

Wie eingangs erwähnt, ist bei der Betrachtung der signifikanten Veränderung der lokalen Umgebung primär nicht der Annäherungsfehler der Approximation 


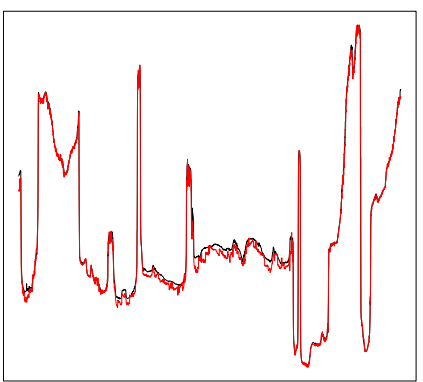

(a) $\theta=1$ (360 Strahlen)

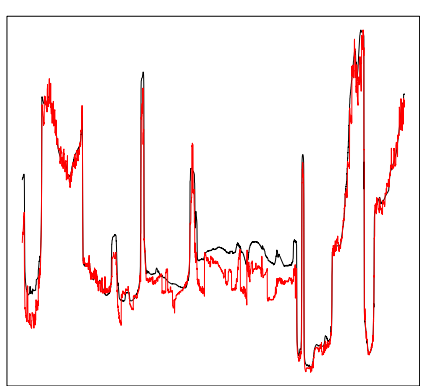

(b) $\theta=5$ (72 Strahlen)

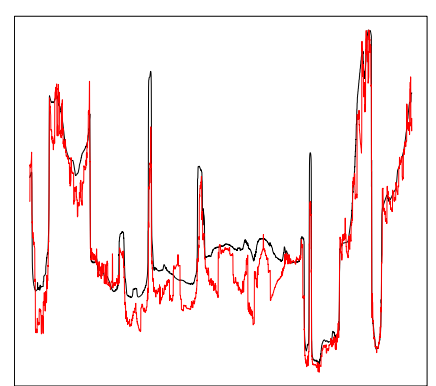

(c) $\theta=10$ (36 Strahlen)

Abbildung 5.12: Einfluss der Anzahl ausgesendeter Strahlen. Dargestellt ist der Vergleich der auf das Intervall $[0,1]$ skalierten exakten Isovisten-Messgröße area $\left(A_{x}\right)$ in schwarz mit der entsprechenden diskreten Isovisten-Messgröße in $\operatorname{rot}(\gamma=\infty, \theta=$ $\{1,5,10\})$.

interessant, sondern die Frage, ob die Werte über die Zeit mit denen der exakten Isovisten zusammenhängen. Aus diesem Grund wird im Folgenden der Korrelationskoeffizient betrachtet, um den Grad des linearen Zusammenhangs zwischen den diskreten und exakten Isovisten-Messgrößen zu untersuchen.

In Abbildung 5.13 ist nun die Pearson-Korrelation [37] zwischen den exakten und den diskreten Isovisten-Messgrößen für verschiedene Anzahlen von ausgesendeten Strahlen dargestellt. Der Parameter $\theta$ wurde mit Werten belegt, sodass eine ganzzahlige Anzahl an auszusendenden Strahlen erstellt wird. Abbildung 5.13a zeigt die Korrelationskoeffizienten ( $y$-Achse) für verschiedene Werte von $\theta$ ( $x$-Achse) für einen fixierten Parameter $\gamma=\infty$. Die Messgröße area ist mit schwarzen Quadraten dargestellt, variance mit roten Kreisen, skewness mit grünen Dreiecken und circularity mit blauen Kreuzen. Abbildung 5.13b ist identisch, nur dass der Parameter der maximalen Strahlenlänge variiert wurde $(\gamma=300)$.

Der grundsätzliche Trend in beiden Abbildungen ist, dass je mehr Strahlen ausgesendet werden, desto stärker ist die Korrelation zwischen den exakten und diskreten Isovisten-Messgrößen. Für eine Belegung von $\theta$ mit Werten zwischen 1 und 6, also der Verwendung von 360, 180, 120, 90, 72 und 60 Strahlen, ist eine sehr hohe und recht konstante Korrelation in Abbildung 5.13a zu erkennen. Bei Begrenzung der Strahlenlängen ergibt sich ein ähnlich stabiles Ergebnis, nur auf einem etwas niedrigeren Niveau mit differenzierteren Werten (Abbildung 5.13b). Die stärkste Korrelation liegt bei der Annäherung der Messgröße area $\left(A_{x}\right)$ vor, und auch das Verhalten der variance ist stark korreliert. Die Korrelation zwischen der Messgröße skewness fällt mit zunehmendem Wert von $\theta$ stärker ab als bei den anderen Messgrößen. Außerdem ist in diesem Fall der strukturierte Fehler stärker ausgeprägt als bei den anderen Messgrößen. Ein niedrigeres Niveau der Korrelation ist in beiden Abbildungen bei $\theta=\{4,8,12\}$ zu erkennen, also bei 90, 45 und 30 Strahlen. Eine mögliche 


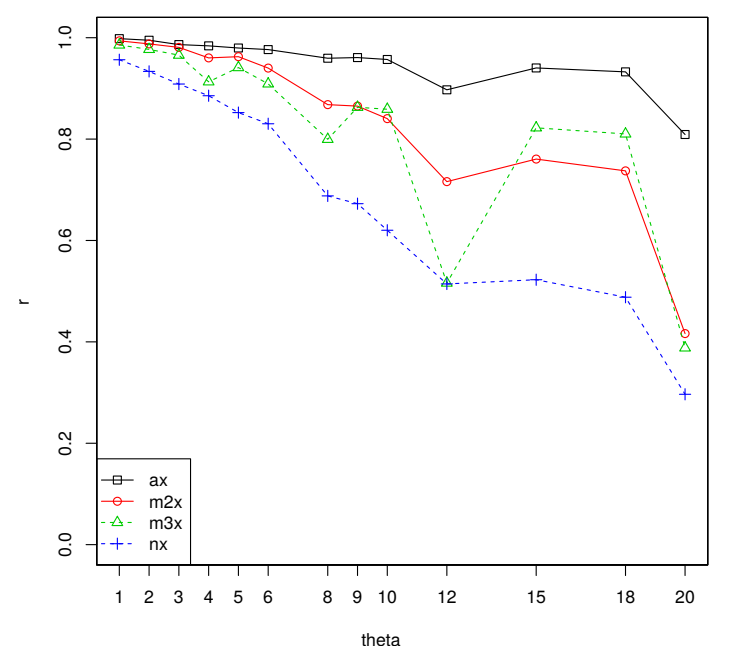

(a) $\gamma=\infty$

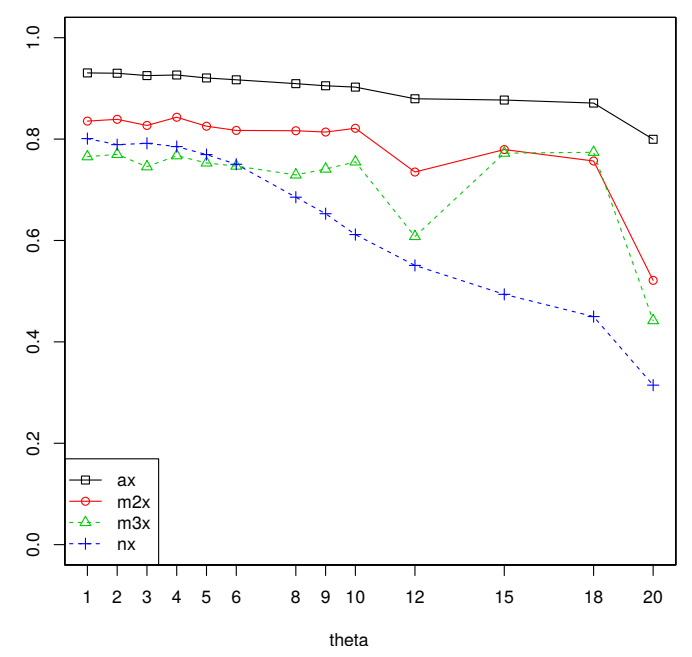

(b) $\gamma=300$

Abbildung 5.13: Korrelation zwischen den exakten und diskreten IsovistenMessgrößen für eine unterschiedliche Anzahl an Strahlen. Messgröße area ist mit schwarzen Quadraten abgebildet, variance mit roten Kreisen, skewness mit grünen Dreiecken und circularity mit blauen Kreuzen.

Ursache für diesen Sachverhalt könnte die „symmetrische“ Ausstrahlung der Strahlen sein. Vermutlich wäre eine „unsymmetrische“ Anordnung an Strahlen für die Verwendung der eher rechtwinkligen Route im Gebäude mit rechtwinkliger Raumanordnung passender. Schließlich ist bei der Messgröße circularity das niedrigste Korrelationsniveau und auch der stärkste Abfall der Korrelation zu beobachten. Dies kann daher rühren, dass circularity mittels zweier Teilformeln berechnet wird, die beide von der Anzahl der Strahlen abhängt: Flächeninhalt und Umfang. Insbesondere das Beschränken der maximalen Strahlenlänge hat eine negative Auswirkung auf die Korrelation (siehe Abbildung 5.13b). Dies ist der Tatsache geschuldet, dass ein Beschneiden der Strahlen in einer Kreisform mündet, auch wenn die Form des Isovisten im Vorfeld weniger kreisförmig war.

Zusammengefasst kann gesagt werden, dass diskrete Isovisten als approximative Beschreibung der lokalen Umgebung verwendet werden können. Durch die unterschiedlichen Skalen der exakten und diskreten Isovisten-Messgrößen sind potentiell zwar hohe Fehler anzunehmen, jedoch ist eine starke Korrelation der Werte über die Zeit gegeben, was zur Annotation von Routen ausreicht. Ebenso wurde die naheliegende Vermutung bestätigt, dass eine höhere Anzahl an Strahlen eine bessere Annäherung an die Messgrößen der exakten Isovisten bewirkt. Die Ergebnisse zeigen jedoch auch, dass schon die Verwendung von 60 Strahlen pro Isovist eine ausreichend gute Annäherung darstellt. 


\subsection{Archetypische Routen und Regionen unter Einbeziehung der lokalen Umgebung}

Dieser Abschnitt behandelt die kombinierte Verwendung von bereits vorgestellten Ansätzen. Die Idee ist die Erweiterung des Systems zur Berechnung archetypischer Routen (Abschnitt 4.4) dahingehend, dass die lokale Umgebung in Form von Isovisten-Messgrößen (Abschnitt 5.3) dazu verwendet wird, archetypische Routen und sogenannte archetypische Regionen zu erstellen.

Die Motivation hinter der Idee ist die Umkehrung der ursprünglichen Vorgehensweise. Anstatt pixelbasiert Werte von Routen zu nehmen, um diese zu gruppieren, werden nun mittels Clusteranalyse die Pixel an sich in Gruppen eingeteilt. Das Ziel ist, Erkenntnisse über die Gebäudestruktur zu erhalten, indem eine Klassifizierung von Regionen in Klassen wie etwa Eingangsbereich, Korridor, Halle oder Straße ermöglicht wird. Die strukturellen Unterschiede innerhalb eines Gebäudes können wiederum als Eingabe für die Berechnung archetypischer Routen verwendet werden. Das bedeutet, dass Routen unterschiedliche Regionen durchlaufen, um dann als Eingabe für die archetypische Analyse zu dienen.

Darüber hinaus ist eine weitere Motivation für den vorliegenden Abschnitt das Erweitern des Systems zur Berechnung archetypischer Routen um Isovisten-Messgrößen. Es sollen archetypische Routen ermittelt werden, die allein auf der Geometrie der Umgebung basieren und nicht mehr nur bezüglich der Geometrie der Routen selbst berechnet werden. Gleichzeitig soll der Effekt der Isovisten-Messgrößen auf die Berechnung der Archetypen untersucht werden. Die Berechnung alternativer Routen auf Grundlage der räumlichen Wahrnehmung kann schließlich dazu verwendet werden, um ausgehend von den Routen die zugrunde liegende Karte zu annotieren.

Zusammengefasst behandelt der vorliegende Abschnitt die vierte der in Abschnitt 5.1 vorgestellten Herausforderungen, nämlich die Identifizierung archetypischer Regionen im Sinne der Analyse von Gebäudestrukturen.

\subsubsection{Konzept}

Die Idee der archetypischen Routen und Regionen unter Einbeziehung der lokalen Umgebung verbindet zwei unterschiedliche Ansätze: Isovisten-Analyse und archetypische Analyse. Der zuerst genannte Ansatz bezieht sich auf die quantitative Untersuchung der visuellen Wahrnehmung von Raum und wurde im vorliegenden Kapitel erläutert und verwendet (Abschnitt 5.2.2). Der zweite Ansatz wurde in Abschnitt 4.2.2 eingeführt und verwendet zur Verfügung gestellte Merkmale von Beobachtungen, um daraus extreme Konstellationen, sogenannte Archetypen, abzuleiten. In diesem Abschnitt werden diese Merkmale die Isovisten-Messgrößen sein. Das Ziel des hier vorgestellten Konzepts besteht aus zwei Teilen: Zum einen sollen archetypische Routen berechnet werden, die sich nicht mehr nur auf die Geometrie der Routen selbst beziehen, sondern auf 


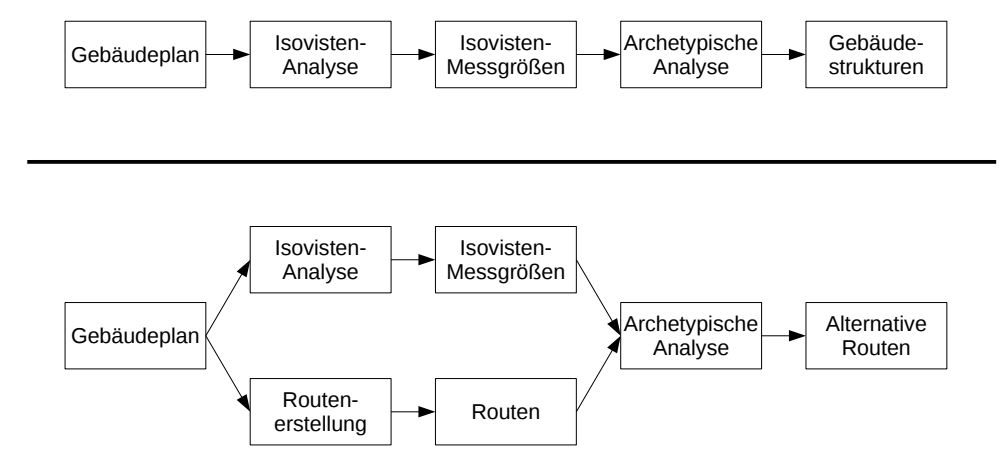

Abbildung 5.14: Ablaufplan für die Analyse eines gegebenen Gebäudeplans (oben) sowie einer Menge von Routen (unten).

die Geometrie der durchlaufenen Umgebung. Zum anderen soll eine Methode zur funktionellen Segmentierung von Raum innerhalb von Gebäudeplänen entstehen. Dies beinhaltet die Unterteilung sowohl von Bereichen innerhalb eines Gebäudes als auch der Umgebung in Klassen wie etwa Eingangsbereich, Gang, Halle oder Straße.

Die eigentliche Untersuchung des Gebäudeplans und der Routen - jeweils repräsentiert in einem multidimensionalen Merkmalsraum - findet mittels einer Erweiterung des Systems zur Berechnung archetypischer Routen statt (siehe Abschnitt 4.4.3). Die als Eingabe verwendeten Routen werden mittels des in Abschnitt 3.3.2 vorgestellten Penalty-Algorithmus berechnet. Abbildung 5.14 zeigt einen Überblick über den Ablauf des Algorithmus.

\subsubsection{Anforderungen an die Eingabe}

Das System zur Berechnung archetypischer Routen nimmt eine 2DRepräsentation einer komplexen Umgebung, beispielsweise einen Gebäudeplan mit angrenzenden Straßen, als Eingabe entgegen. Polygone repräsentieren begehbare Flächen. Die Begrenzungen und inneren Löcher der Polygone stellen Einschränkungen wie etwa Wände, Installationen oder andere Hindernisse dar. Um Isovisten-Felder für die vollständige Beschreibung der Umgebung zu berechnen, wird der Gebäudeplan mittels einer regelmäßigen GitterParkettierung (siehe Abschnitt 2.3.2) gerastert und das geometrische Zentrum einer Zelle als repräsentativer Punkt gewählt. Die Auflösung des Rasters muss angemessen gewählt werden, um die Eigenschaften der Umgebung sinnvoll einfangen zu können. Eine zu grobe Auflösung kann bewirken, dass Änderungen der Werte der Isovisten-Messgrößen an Übergängen wie etwa Türen oder Abbiegungen verpasst werden. Im Folgenden wird standardmäßig eine Auflösung von 0,5 Metern gewählt. Eine feinere Auflösung würde zwar mehr Details der Umgebung widerspiegeln, jedoch führt dies zu unnötig höheren Berechnungskosten im Sinne von Zeit und Speicher. Außerdem ist 0,5 Meter eine angemesse- 
ne Annäherung für die für gewöhnlich gewählte Schrittlänge bei der Navigation von Menschen und in Wegfindungsszenarien.

Schließlich wird die zu analysierende Menge von Routen zwischen einem gegebenen Start- und Zielpunkt mittels des Penalty-Algorithmus (siehe Abschnitt 3.3.2) berechnet und zur Verfügung gestellt. Die Routen liegen somit als Vektoren von $x$ - und $y$-Koordinaten vor.

\subsubsection{Clustering von Regionen und Routen}

Zur Berechnung archetypischer Regionen und Routen unter Einbeziehung der lokalen Umgebung wird auf die Isovisten-Messgrößen nach Benedikt zurückgegriffen (siehe Abschnitt 5.2.2 und [15]). Die sechs Messgrößen werden wie beschrieben unter Verwendung des gegebenen Gebäudeplans in der vektorbasierten Darstellung berechnet und anschließend für die weitere Verwendung gerastert.

Ziel des Algorithmus ist die Erzeugung von Erkenntnissen über die Gebäudestruktur sowie die Identifizierung einer kleinen Menge von geeigneten alternativen Routen zwischen zwei gegebenen Punkten. Um dies zu erreichen, müssen der Gebäudeplan, die Isovisten-Messgrößen und die Pfade in eine Form gebracht werden, die von der archetypischen Analyse verarbeitet werden kann.

Bei der Analyse der Gebäudestruktur wird jedes Pixel des Gebäudeplans als Beobachtung angesehen. Es sind somit $N=r \times c$ Beobachtungen gegeben, wobei $r$ die Anzahl der Pixelzeilen und $c$ die Anzahl der Pixelspalten darstellt. Jede Beobachtung hat $m=6$ Attribute $\left\{A_{x}, P_{x}, Q_{x}, M_{2, x}, M_{3, x}, N_{x}\right\}$. Das bedeutet, dass die Eingabe für die archetypische Analyse eine $N \times m$ Matrix $X$ ist, die alle Pixel des Gebäudeplans mit jeweils sechs Isovisten-Messgrößen beinhaltet.

Das Clustering der (großen) Menge von Eingangsrouten mit jeweils gleichem Start- und Zielpunkt verläuft geringfügig anders: Jede Route besteht aus mehreren Pixelkoordinaten, die den Verlauf des Pfades beschreiben. Es wird definiert, dass jede Route eine Beobachtung darstellt und für jedes Routenpixel werden die entsprechenden Isovisten-Messgrößen mittels der zuvor berechneten Matrix ermittelt. Für jede Route und für jede der sechs Isovisten-Messgrößen werden das Minimum, das Maximum, der Durchschnitt, der Median und die Varianz berechnet. Das bedeutet, dass die Eingabe für die archetypische Analyse der Routen eine $N \times m$ Matrix $X$ ist, die alle Routen mit jeweils $5 \times 6=30$ Attributen beinhaltet.

\subsubsection{Evaluation und Diskussion}

Für die Evaluation und Diskussion der präsentierten Vorgehensweise wird erneut der Gebäudeplan des Hauptgebäudes der Technischen Universität München mitsamt der nahen Umgebung (Theresienstraße, Arcisstraße, Gabelsbergerstraße, Luisenstraße) verwendet. Das auch in weiteren Fallstudien wie etwa [126], [127], [130] verwendete Gebäude ist durch seine Komplexität und die 
hohe Anzahl an Eingängen charakterisiert. Beide Eigenschaften ergeben sich dadurch, dass bestimmte Stockwerke über verschiedene Treppenaufgänge betreten werden können und durch die Vielfalt an Möglichkeiten, das Gebäude mit seinen mehr als zwanzig Eingängen zu durchlaufen. Demnach ist eine hohe Anzahl an alternativen Routen zu erwarten. Der ursprüngliche Gebäudeplan wurde vereinfacht, indem die eingezeichneten Türen entfernt wurden, um mit dem Penalty-Algorithmus zur Routenerstellung kompatibel zu sein.

\subsubsection{Identifizierung archetypischer Regionen}

Ziel des vorgestellten Ansatzes ist die Einbeziehung der lokalen Umgebung mittels Isovisten-Messgrößen zur Identifizierung archetypischer Routen, aber auch zur Identifizierung archetypischer Regionen. Somit wird in diesem Abschnitt untersucht, ob und wie funktioneller Raum innerhalb eines gegebenen Gebäudeplans identifiziert werden kann. Das bedeutet, dass - wie in Abschnitt 5.4.1 beschrieben - die archetypische Analyse auf Isovisten-Messgrößen des Gebäudeplans angewendet wird. Jedes begehbare Pixel repräsentiert eine Beobachtung, und die berechneten Isovisten-Messgrößen stellen die Merkmale der Beobachtungen dar.

Wie auch in Abschnitt 4.4 wird der resultierende Annäherungsfehler (residual sum of squares, $R S S$ ) bei der Berechnung der konvexen Hülle um die Beobachtungen im Merkmalsraum betrachtet, um einen geeigneten Wert für die Anzahl der Archetypen $k$ zu erhalten. Für die Evaluation wurde die archetypische Analyse mit Werten von $1 \leq k \leq 10$ durchgeführt. Das Eigenwertediagramm der resultierenden Residuenquadratsumme aus Abbildung 5.15 legt nahe, einen Wert von $k=4, k=5$ oder sogar $k=7 \mathrm{zu}$ wählen. Aus Anwendungssicht birgt ein hoher Wert wie $k=7$ die Gefahr, dass die notwendige Interpretation der Ergebnisse unübersichtlich wird. Aus diesem Grunde wird im weiteren Verlauf der Evaluation die Anzahl der Archetypen auf $k \leq 5$ beschränkt.

Die archetypische Analyse ist - wie in den Abschnitten 4.2.2 und 4.4 erwähnt - ein sogenanntes „fuzzy“ Clusteringverfahren. Das bedeutet, dass jede Beobachtung durch eine konvexe Kombination der identifizierten Archetypen repräsentiert wird und somit mehreren Archetypen zugeordnet werden kann. Aus diesem Grunde werden im Folgenden die Punkte innerhalb des Gebäudeplans diskutiert, die einen bestimmten Grad an Zugehörigkeit zu einem Archetypen besitzen. Wie auch bei den archetypischen Routen ordnen wir eine Beobachtung, in diesem Falle einen Pixel, einem Archetypen zu, wenn der entsprechende Wert in der Koeffizientenmatrix $\alpha$ höher als ein definierter Schwellwert ist.

Wenn der zu analysierende Datensatz mehr natürliche Cluster beinhaltet als vom Algorithmus gefunden werden sollten (zu klein gewähltes $k$ ), so sind die Kombinationen der Beobachtungen nicht sehr aussagekräftig und können somit nicht mehr zur Interpretation der Ergebnisse genutzt werden. Laut dem Eigenwertediagramm aus Abbildung 5.15 sollen vier beziehungsweise fünf Ar- 


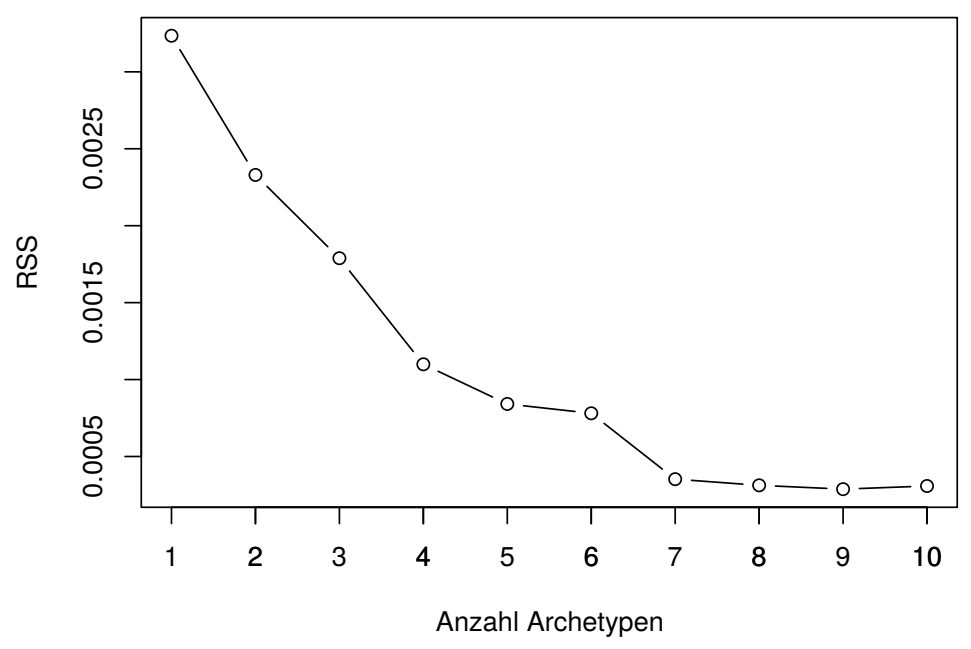

Abbildung 5.15: Eigenwertediagramm der resultierenden $R S S$ für unterschiedliche Werte von $k$.

chetypen den Datensatz gut beschreiben. Der Vollständigkeit halber werden im Folgenden zusätzlich die Ergebnisse für zwei und drei Archetypen diskutiert. Abbildung 5.16a zeigt einen parallelen Koordinatenplot für $k=2$. Auf der Abszisse sind die sechs Isovisten-Messgrößen nebeneinander dargestellt, die grauen Linien repräsentieren die Menge der Beobachtungen (das heißt, eine Linie ist genau ein begehbares Pixel) und die eingefärbten Linien stellen die identifizierten abstrakten Archetypen dar. Im Grunde zeigt der parallele Koordinatenplot die konkrete Ausprägung der Merkmalsmenge der abstrakten Archetypen genau wie ein Säulendiagramm (siehe beispielsweise Abbildung 4.16). Die Beobachtungen sind jedoch zusätzlich im Merkmalsraum eingetragen, wodurch ein erster visueller Eindruck der Beziehung zwischen Archetypen und Datensatz erlangt werden kann. Der rote Archetyp kann als eine Beobachtung zusammengefasst werden, die grundsätzlich hohe Werte der IsovistenMessgrößen besitzt, während der grüne Archetyp eher niedrige Werte besitzt. Lediglich die Schiefe der Strahlenlängen $M_{3, x}$ (skewness) ist in beiden Fällen ähnlich moderat. Generell deuten hohe Werte der Isovisten-Messgrößen wie beim roten Archetypen darauf hin, dass der Standpunkt einen guten Ausblick besitzt (hohe area $A_{x}$ ), was wiederum zur Folge hat, dass viele Oberflächen von Hindernissen und Wänden gesehen werden können (hoher real-surface perimeter $\left.P_{x}\right)$. Im vorliegenden Falle von $k=2$ besitzt der grüne Archetyp recht niedrige Werte. Insbesondere die niedrige Varianz der Strahlen $M_{2, x}$ (variance) impliziert, dass die Aussichtsfläche - also der Isovist selbst - eine eher regelmäßige Form besitzt. Abbildung 5.16b zeigt nun die entsprechenden archetypischen Regionen im Gebäudeplan, indem die Pixel entsprechend ihrer Zuordnung zu den Archetypen eingefärbt wurden. Der Schwellwert für die Zuordnung ist für den vorliegenden Fall auf $\alpha>0,6$ gesetzt. Die Darstellung ist 


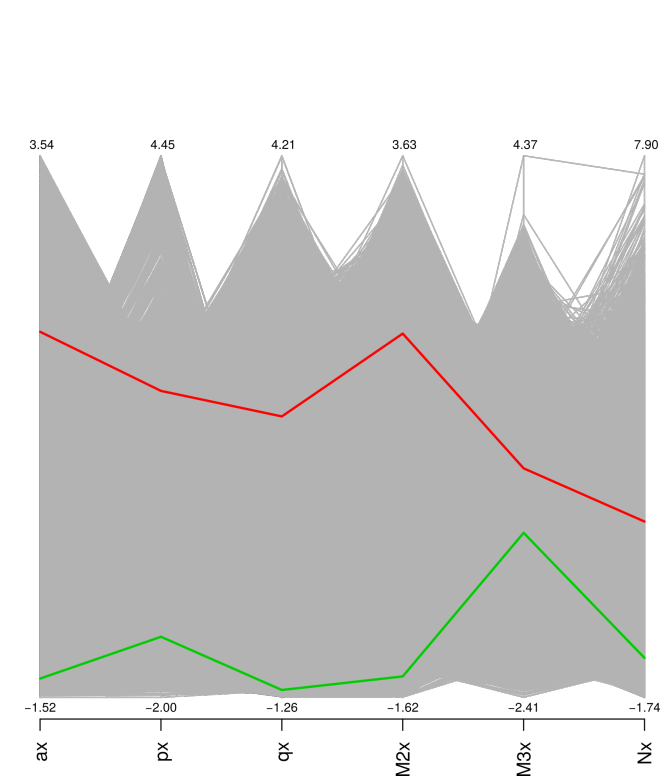

(a) Paralleler Koordinatenplot

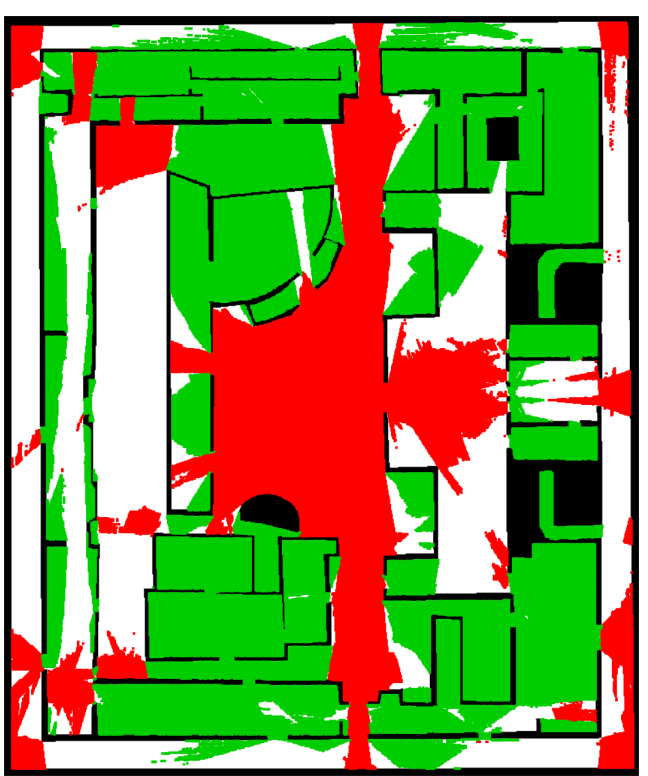

(b) Archetypische Regionen

Abbildung 5.16: Evaluationsergebnisse für $k=2$. Beobachtungen mit einem entsprechenden Koeffizienten $\alpha>0,6$ werden als „Top-Pixel“ angesehen und somit einer archetypischen Region zugeordnet.

kohärent zur soeben aufgeführten Interpretation: Grundsätzlich repräsentieren die roten Pixel Teile des Gebäudeplans, in denen viel Fläche eingesehen werden kann. Die grünen Pixel verhalten sich gegenteilig, dort ist die Sicht stark eingeschränkt.

Abbildung 5.17 zeigt die Ergebnisse für $k=3$. Im parallelen Koordinatenplot (Abbildung 5.17a) ist zu erkennen, dass der grüne Archetyp eine ähnliche Gestalt wie bei $k=2$ besitzt, dieses mal jedoch mit noch geringeren Werten. Insbesondere durch die niedrige Varianz $M_{2, x}$ (variance) ist eine regelmäßige Form der entsprechenden Regionen zu erwarten. Auch der rote Archetyp besitzt wieder ein durchgängig hohes Niveau an Werten, die jedoch extremen Schwankungen ausgesetzt sind. Der Flächeninhalt des Isovisten $A_{x}$ (area) ist extrem hoch und lässt einen guten Ausblick vermuten. Die moderat vielen sichtbaren Wände $P_{x}$ (real-surface perimeter), die hohe Verdeckung $Q_{x}$ (occlusivity) und die hohe Varianz $M_{2, x}$ (variance) deuten auf eine facettenreiche Sichtlinie hin. Erneut scheint dieser Archetyp für eine geräumige Fläche zu stehen. Der neue, blaue Archetyp zeigt eine interessante Merkmalsausprägung. Die moderat große sichtbare Fläche $A_{x}($ area $)$, die vielen einsehbaren Hindernisse und Wände (hohes $P_{x}$ ) und der sehr niedrige Wert der Verdeckung $Q_{x}$ (occlusivity) suggerieren eine regelmäßige und einfache Struktur ohne viel Unsicherheit. Wenn dieser initialen Interpretation gefolgt und der entsprechend eingefärbte Gebäudeplan in Abbildung 5.17b betrachtet wird (,Top-Pixel“ mit $\alpha>0,5)$, so repräsentieren die recht kompakten roten Pixel freien Raum mit 


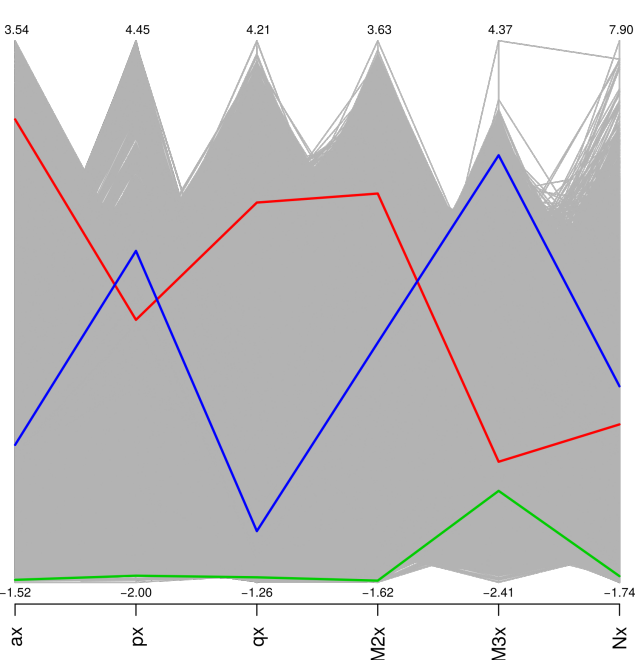

(a) Paralleler Koordinatenplot

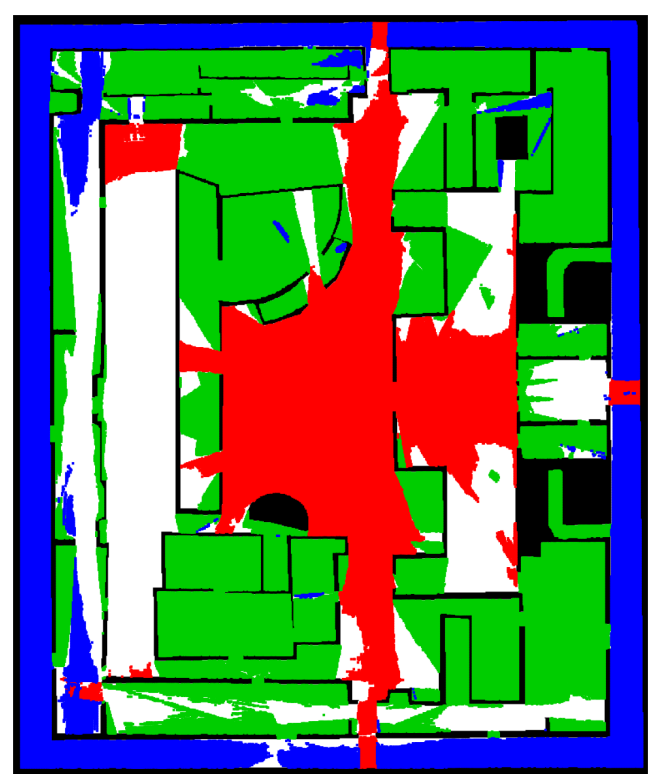

(b) Archetypische Regionen

Abbildung 5.17: Evaluationsergebnisse für $k=3$. Beobachtungen mit einem entsprechenden Koeffizienten $\alpha>0,5$ werden als „Top-Pixel“ angesehen und somit einer archetypischen Region zugeordnet.

weiter Aussicht. Die neue, blaue archetypische Region kann derart interpretiert werden, dass solche Punkte in der Karte eine stark begrenzte Aussicht in zwei Richtungen besitzen und eine sehr weite Aussicht in die entsprechend anderen Richtungen. Dies ist im vorliegenden Fall bei den Straßen gegeben, die das Gebäude umlaufen. Die grüne archetypische Region hat sich im Grunde nicht verändert, sie kann erneut als Fläche interpretiert werden, in der die Sicht eingeschränkt und regelmäßig ist, wie etwa in Räumen oder kleineren Hallen.

Schließlich sollen die weißen Flächen in Abbildung 5.17b hervorgehoben werden, da sie einen interessanten Effekt der archetypischen Analyse widerspiegeln. Diese Pixel sind nicht eingefärbt, da die entsprechenden Werte der Koeffizientenmatrix $\alpha$ unter dem definierten Schwellwert von 0,5 liegen. Das bedeutet, dass die Beobachtungen schwer mittels der identifizierten Archetypen zu beschreiben sind (sie sind im Merkmalsraum weit von den Archetypen entfernt). Wenn die große weiße Fläche auf der linken Seite des Gebäudeplans betrachtet wird, so ist ersichtlich, dass hier die Eigenschaften aller drei Archetypen anzutreffen sind: Gewissermaßen ist die Fläche ähnlich einem Raum, da sie eine regelmäßige Form und eine eingeschränkte Sicht besitzt (grüner Archetyp). Außerdem ähnelt sie einer offenen Freifläche aufgrund ihres recht großen Ausmaßes (roter Archetyp). Und schließlich erinnert sie auch an einen Gang oder eine Straße aufgrund ihrer schmalen und länglichen Form (blauer Archetyp).

Abbildung 5.18 stellt die archetypischen Regionen basierend auf der archety- 


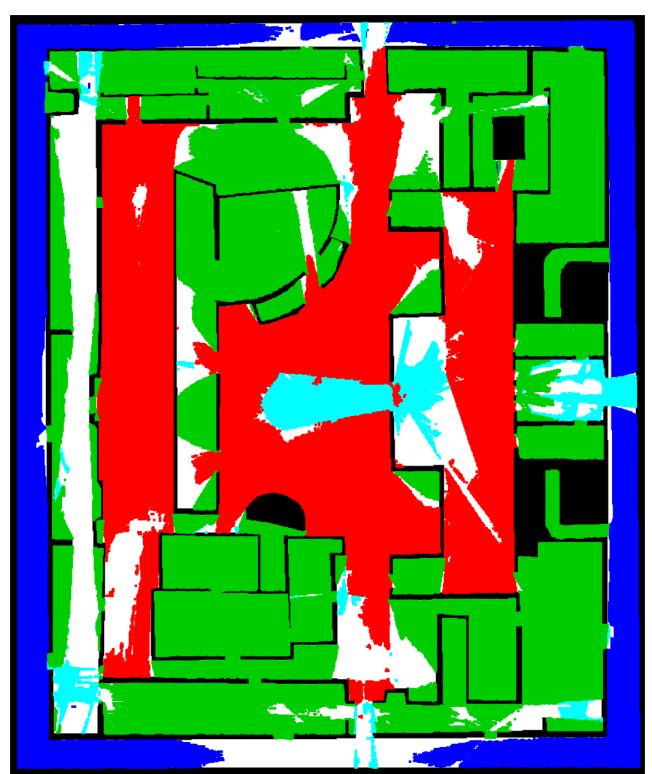

(a) $k=4$

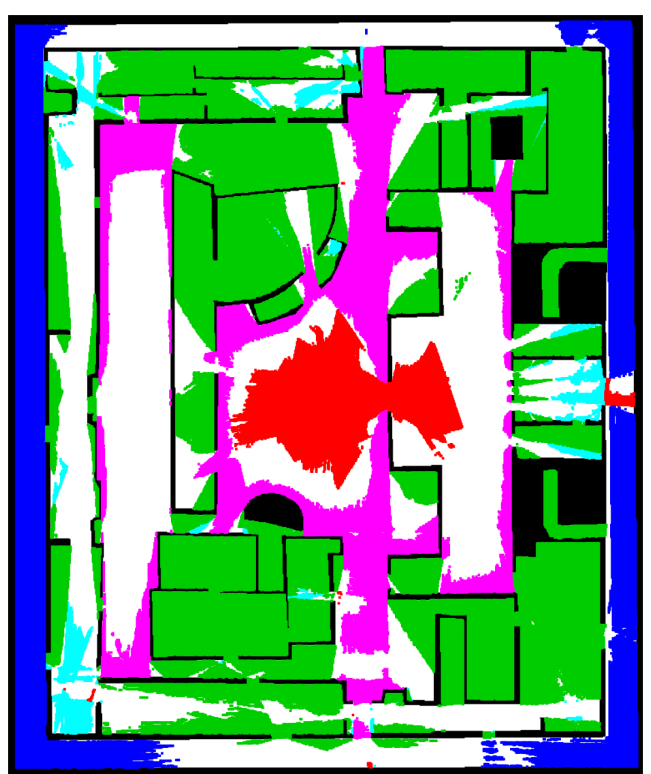

(b) $k=5$

Abbildung 5.18: Archetypische Regionen für $k=4$ und $k=5$. Für die Zuordnung eines Pixels zur archetypischen Region wird ein Schwellwert von $\alpha>0,5$ verwendet.

pischen Analyse mit $k=4$ (Abbildung 5.18a) und $k=5$ (Abbildung 5.18b) dar. Die Wahl dieser Werte ist laut dem Eigenwertediagramm aus Abbildung 5.15 für den gegebenen Gebäudeplan am besten geeignet. Die entsprechenden parallelen Koordinatenplots (nicht abgebildet) sowie eine visuelle Untersuchung der Pixel deuten darauf hin, dass der grüne Archetyp („Räume“) und der blaue Archetyp („Gang oder Straße“) nahezu identisch geblieben sind (in Bezug auf $k=3$ ), nur die Interpretation der „offenen Freifläche“ ist weiter unterteilt. Der rote Archetyp weist weiterhin auf Flächen mit einer weiten Aussicht hin, jedoch existiert nun ein türkiser Archetyp, der zusätzlich eine tiefe Sicht mit viel Verdeckung in beispielsweise Eingänge besitzt (sehr hohe occlusivity $Q_{x}$ ). Abbildung 5.18b verfeinert diese Struktur mit pinkfarbenen archetypischen Regionen, bei denen ein Beobachter viel Fläche des Gebäudes einsehen kann, jedoch mit einer Wand im Rücken.

\subsubsection{Identifizierung archetypischer Routen}

Nach der Identifizierung archetypischer Regionen wird in diesem Abschnitt analysiert, welchen Effekt Isovisten-Messgrößen bei der Ermittlung archetypischer Routen zwischen zwei gegebenen Punkten haben. Zu diesem Zweck wurden mehrere Experimente durchgeführt und für unterschiedliche Paare von Start- und Zielpunkten Mengen von Routen erstellt. Hierzu wurde der PenaltyAlgorithmus verwendet (Abschnitt 3.3.2).

Im Folgenden wird ein repräsentatives Ergebnis für eine Menge von 400 


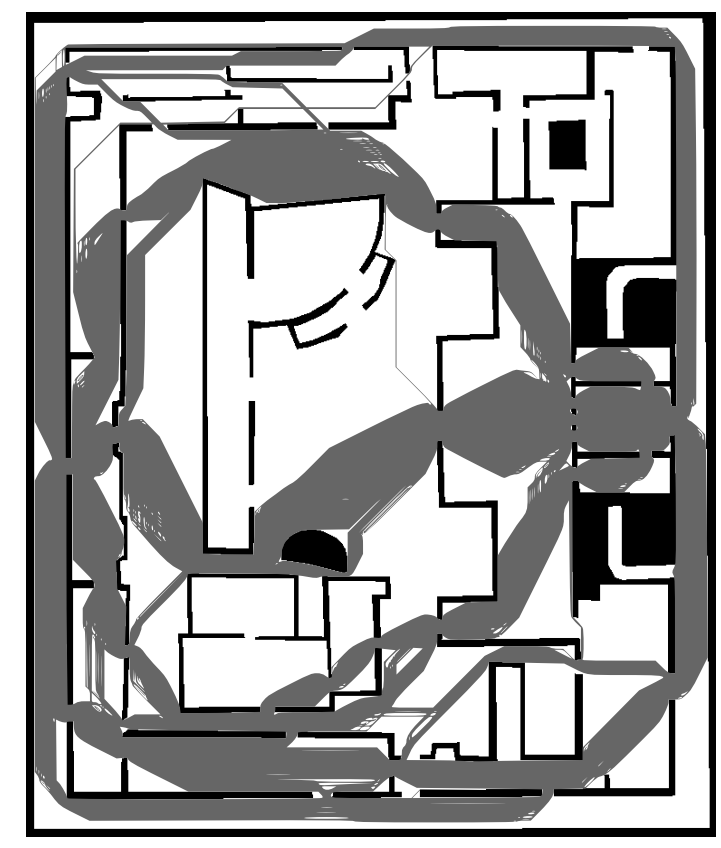

Abbildung 5.19: Der verwendete Gebäudeplan und die berechnete Menge von 400 Routen vom Haupteingang des Gebäudes (rechte Seite) zum gegenüberliegenden Eingang.

Routen verwendet, die vom Haupteingang des Gebäudes (rechte Seite) bis zu einem weiteren Eingang auf der gegenüberliegenden Seite des Gebäudes führen. Abbildung 5.19 zeigt den verwendeten Gebäudeplan und die resultierenden Routen, die das Gebäude in unterschiedlichen Variationen durch- und umlaufen. Darunter findet sich unter anderem das vollständige Umgehen des Gebäudes, das Durchlaufen unterschiedlicher Räume sowie das Durchqueren des Innenhofs. Wie bereits angesprochen wird jede der 400 Routen als Beobachtung angesehen und für jede der sechs Isovisten-Messgrößen werden Minimum, Maximum, Mittelwert, Median und Varianz über alle Punkte einer Route berechnet. Mittels dieser Daten wird versucht, die hohe Anzahl an partiell sehr ähnlichen Routen auf eine niedrige Zahl von stark unterschiedlichen Routen $\mathrm{zu}$ reduzieren.

Wie auch bei der einführenden Erläuterung der archetypischen Routen und der archetypischen Distanz in Abschnitt 4.4 wird das Eigenwertediagramm der $R S S$ konsultiert, um einen geeigneten Wert für $k$ zu erhalten (siehe Abbildung 5.20). Es ist eine starke Krümmung bei $k=3$ zu erkennen, was aus Sicht einer Anwendung einen angemessen Wert darstellt.

Wie in Abschnitt 4.4.1 erläutert müssen die berechneten abstrakten Archetypen nicht zwingend existieren oder beobachtbar sein. Aus diesem Grunde werden die abstrakten archetypischen Routen mittels einer Nächster-NachbarSuche realisiert (Abschnitt 4.4.1). Abbildung 5.21 visualisiert die entsprechenden realisierten archetypischen Routen für $2 \leq k \leq 5$.

Abbildung 5.21a zeigt das Ergebnis für $k=2$. Die rote Route durchläuft das 


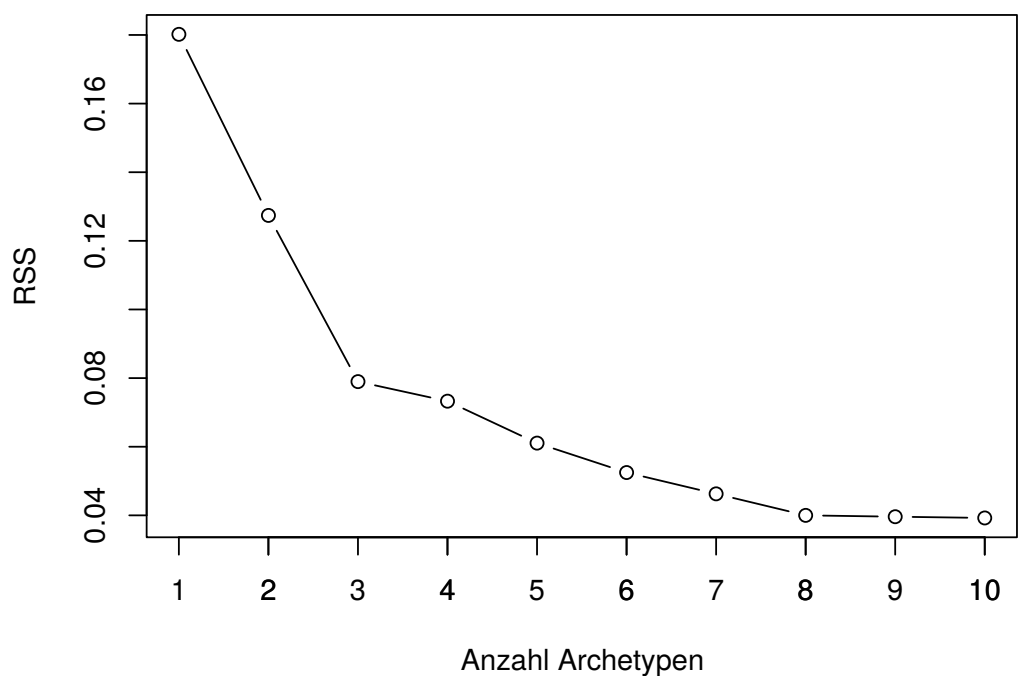

Abbildung 5.20: Eigenwertediagramm der resultierenden $R S S$ für unterschiedliche Werte von $k$ für den Gebäudeplan und die Routen aus Abbildung 5.19.

Gebäude relativ mittig, während sie im Innenhof eine weite Sicht besitzt und sich zum Ende hin kurvig und windend bewegt. Die grüne archetypische Route ist im Grunde das komplette Gegenteil. Sie beschreibt einen geradlinigen Pfad mit wenigen Abbiegungen und einer eher eingeschränkten Sicht. Die Route folgt der Straße um das Gebäude herum und durchläuft einige Räume im nördlichen und westlichen Teil des Gebäudes.

Das Ergebnis für $k=3$ ist in Abbildung 5.21b zu sehen. Die rote archetypische Route verläuft erneut relativ kurvig durch die Freifläche mit einer hohen Aussicht. Die grüne archetypische Route hat sich ein wenig verändert: sie ist nun „extremer“ in der Hinsicht, dass sie nun stark geradlinig verläuft und nur eine minimale Anzahl an Abbiegungen besitzt. Die Aussicht ist stark eingeschränkt mit sehr wenig „Unsicherheit“ durch Verdeckung. Die neue blaue Route ist recht komplex: sie durchläuft das Gebäude sehr facettenreich und nutzt enge Stellen, durch die Räume, Türen und Gänge durchquert werden.

Bei der Berechnung von $k=4$ Archetypen (siehe Abbildung 5.21c) ergeben sich die gleichen drei Routen wie bei $k=3$. Zusätzlich wird die türkise archetypische Route ausgegeben, die als eine Art Mischung aus grünem und blauem Archetyp angesehen werden kann. Die türkise Route ist recht geradlinig mit wenig Abbiegungen, jedoch durchläuft sie eher Räume und Türen anstatt die Straße, wie es die „extremere“ grüne Route tut.

Schließlich ist in Abbildung 5.21d das Ergebnis für $k=5$ abgebildet. Die berechneten Routen sind identisch zu denen bei $k=4$, jedoch ist die neue pinkfarbene archetypische Route enthalten. Sie ist sehr ähnlich zur roten Route, die ebenfalls den Innenhof durchquert, jedoch umläuft die pinkfarbene Route das isolierte Gebäude im Innenhof nördlich statt südlich. 


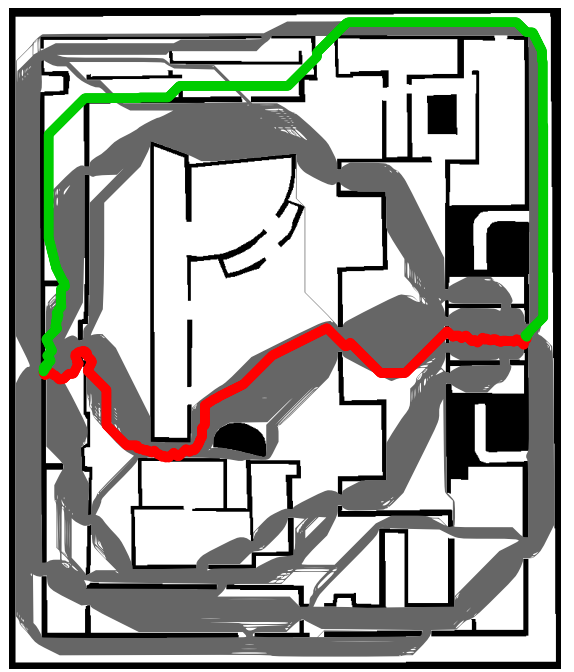

(a) $k=2$

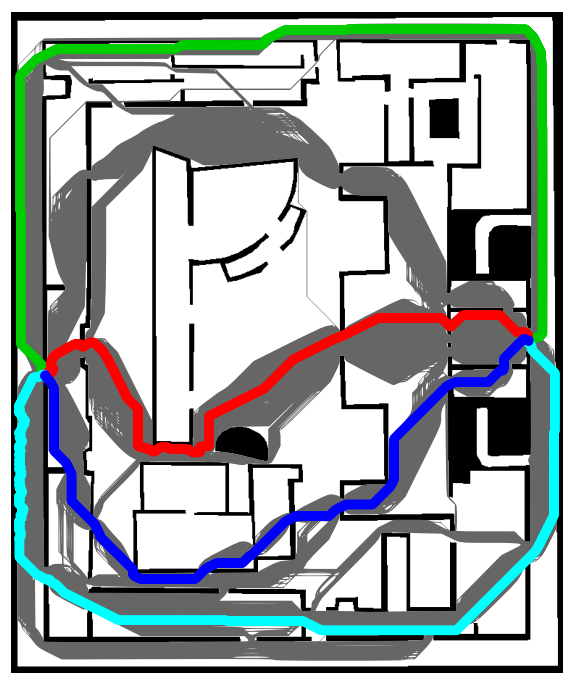

(c) $k=4$

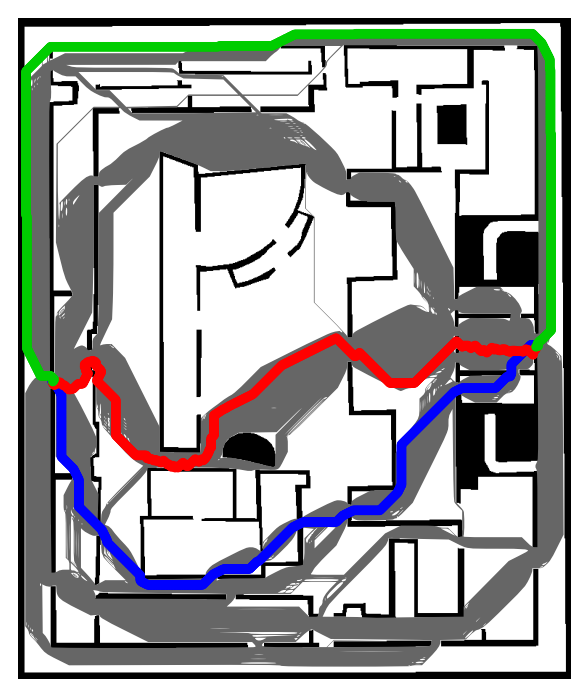

(b) $k=3$

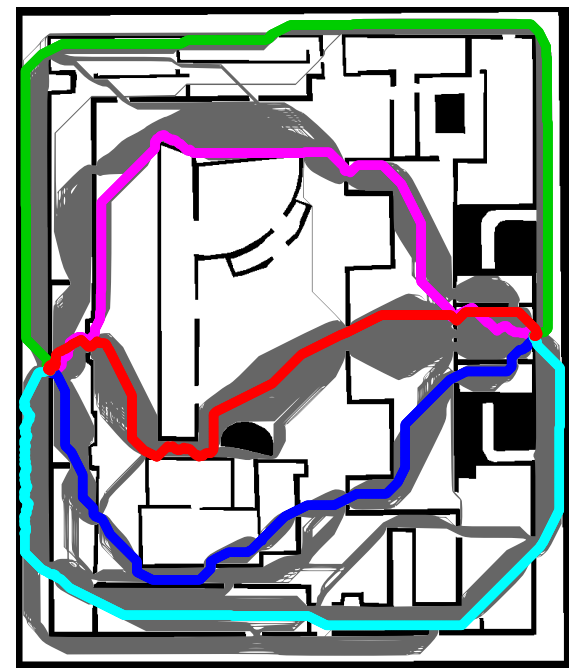

(d) $k=5$

Abbildung 5.21: Realisierte archetypische Routen für $2 \leq k \leq 5$. 
Zusammengefasst kann gesagt werden, dass alle fünf Routen geeignete Alternativen mit sehr unterschiedlichen Eigenschaften darstellen. Weitere Versuche haben gezeigt, dass ab $k=6$ die resultierenden Routen beginnen, sich zu überlagern. Das bedeutet, dass es zwar eine große Distanz im Merkmalsraum gibt, die Routen jedoch im Datenraum sehr ähnlich sind.

\subsubsection{Mengen archetypischer Routen}

Das alleinige Fokussieren auf einen einzigen Repräsentanten einer abstrakten archetypischen Route kann potentiell die Interpretation verfälschen. Im Folgenden werden daher alle Beobachtungen betrachtet, die eine bestimmte Nähe zu den berechneten Archetypen besitzen. Dies wird - wie in Abschnitt 4.4.2 beschrieben - mittels der archetypischen Distanz behandelt, wobei eine Beobachtung „nah“ einem Archetypen ist, wenn der entsprechende Eintrag in der Koeffizientenmatrix $\alpha$ einen gewissen Schwellwert überschreitet.

In Abbildung 5.22 wird dies für $k=3$ und einen Schwellwert von $\alpha>0,8$ gezeigt. Die dem grünen Archetypen zugeordneten Routen durchlaufen alle den Innenhof, während sie mehrere Variationen am Anfang und am Ende der Route besitzen. Die Routen des blauen Archetyps sind charakterisiert durch den geradlinigen Verlauf durch die Straßen um das Gebäude herum und die langen, engen Gänge. Diese können nicht nur im oberen Bereich des Gebäudeplans gefunden werden, sondern auch in Variationen im unteren Bereich der Karte. Die Varianten der Routen - seien es Abkürzungen oder Umwege - finden stets an engen Stellen statt. Schließlich zeigen auch die dem roten Archetypen zugeordneten Routen eine gewisse Varianz, wie Räume und Türen durchlaufen werden. Eine gemeiname Eigenschaft der roten Routen ist, dass sie einen eher umständlichen Verlauf besitzen. Es ist somit klar ersichtlich, dass die identifizierten Mengen von archetypischen Routen auf dem Eindruck der lokalen Umgebung beim Durchlaufen des Gebäudes basieren und nicht ausschließlich auf der geographischen Lage.

Zusammengefasst kann gesagt werden, dass die resultierenden Mengen von Routen in Anwendungsfällen verwendet werden können, in denen Vielfalt und Auswahl wünschenswert sind. Ein Beispiel sind Computerspiele, in denen Nicht-Spieler-Charaktere - basierend auf einer gewissen Strategie - eine Menge archetypischer Routen (Top-Routen) generieren und aus dieser Menge zufällige Routen auswählen, um den menschlichen Spieler zu überraschen. Ein weiterer Anwendungsfall sind Navigationssysteme für Fußgänger, die nichtdeterministisch Routen empfehlen und somit proaktiv Engpässe verhindern.

\subsubsection{Visuelle Analyse der Messgrößen archetypischer Routen}

Die Eigenschaften der gefundenen archetypischen Routen können weiter interpretiert werden, indem die Isovisten selbst an den von den Routen durchlaufenen Stellen untersucht werden. Für eine klare und einfache Erläuterung soll das Ergebnis der realisierten archetypischen Routen für $k=2$ dienen (siehe 


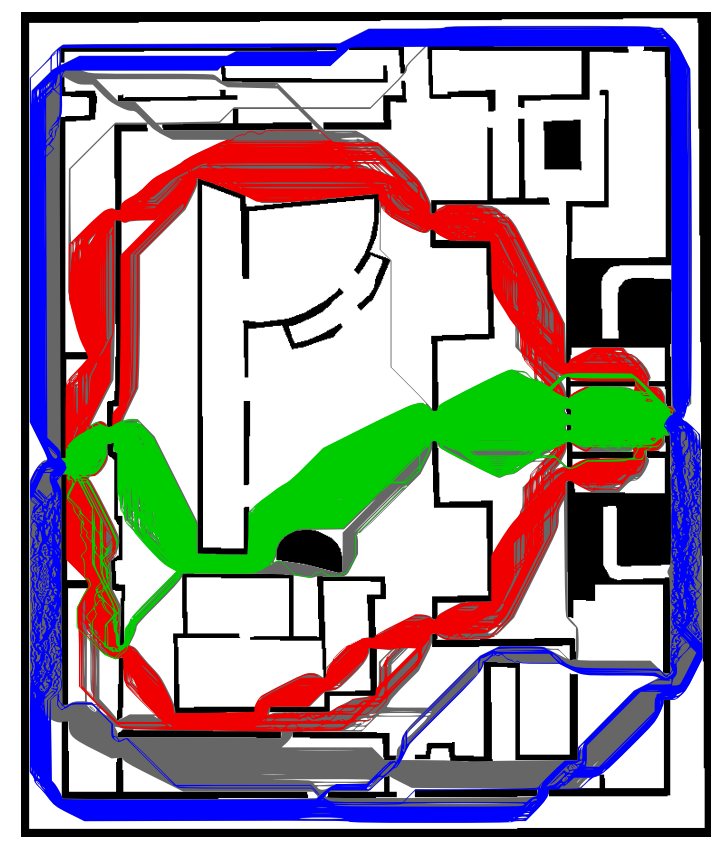

Abbildung 5.22: Top-Routen für $k=3$, das heißt es sind Beobachtungen mit einem entsprechenden Wert des Koeffizienten $\alpha>0,8$ farbig eingezeichnet.

Abbildung 5.21a). In Abbildung 5.23 sind links die realisierten archetypischen Routen für $k=2 \mathrm{zu}$ sehen sowie mittig und rechts jeweils zwei alternative Darstellungen zur visuellen Analyse.

Für die mittlere Darstellung wurden die Isovisten-Messgrößen der Routen normalisiert und in ein gestapeltes Flächendiagramm eingezeichnet. Die $x$ Achse ist dabei als zeitlicher Verlauf einer Route anzusehen, beginnend bei 0 mit dem Start der Route am Haupteingang im rechten Bereich des Gebäudes und endend nach etwa 850 beziehungsweise 1450 Zeitschritten am Ziel der Route im gegenüberliegenden Bereich des Gebäudes. Für die rote Route ist im gestapelten Flächendiagramm das Durchschreiten der drei Türen als starker Abfall der Messgrößenwerte zu erkennen (bei etwa 90, 280 und 770). Ebenso ist das Durchqueren des Innenhofs durch das hohe Niveau von $A_{x}$ zu erkennen (türkise Fläche etwa zwischen 300 und 580). Für die grüne Route sind insbesondere die Spitzen der Messwerte im Flächendiagramm auffallend, die an Stellen des Gebäudeplans entstehen, an denen nach langer eingeschränkter Sicht an Türen oder Eingängen vorbeigelaufen wird, sodass ein weiter Blick in das Gebäudeinnere möglich ist.

Die rechte Darstellung in Abbildung 5.23 beinhaltet alle Isovisten, die von der jeweiligen Route aus beobachtet werden. Die Standpunkte der Isovisten werden dabei gestapelt, um eine Radar-ähnliche Sicht zu erlangen. Diese Visualisierung ist verwandt mit dem Minkowski-Modell [15], [143], bei dem Isovisten ebenfalls übereinander gelegt, jedoch die Standpunkte im Gebäudeplan beibehalten werden. Durch das Stapeln wird die Zeitdimension aufgegeben, 

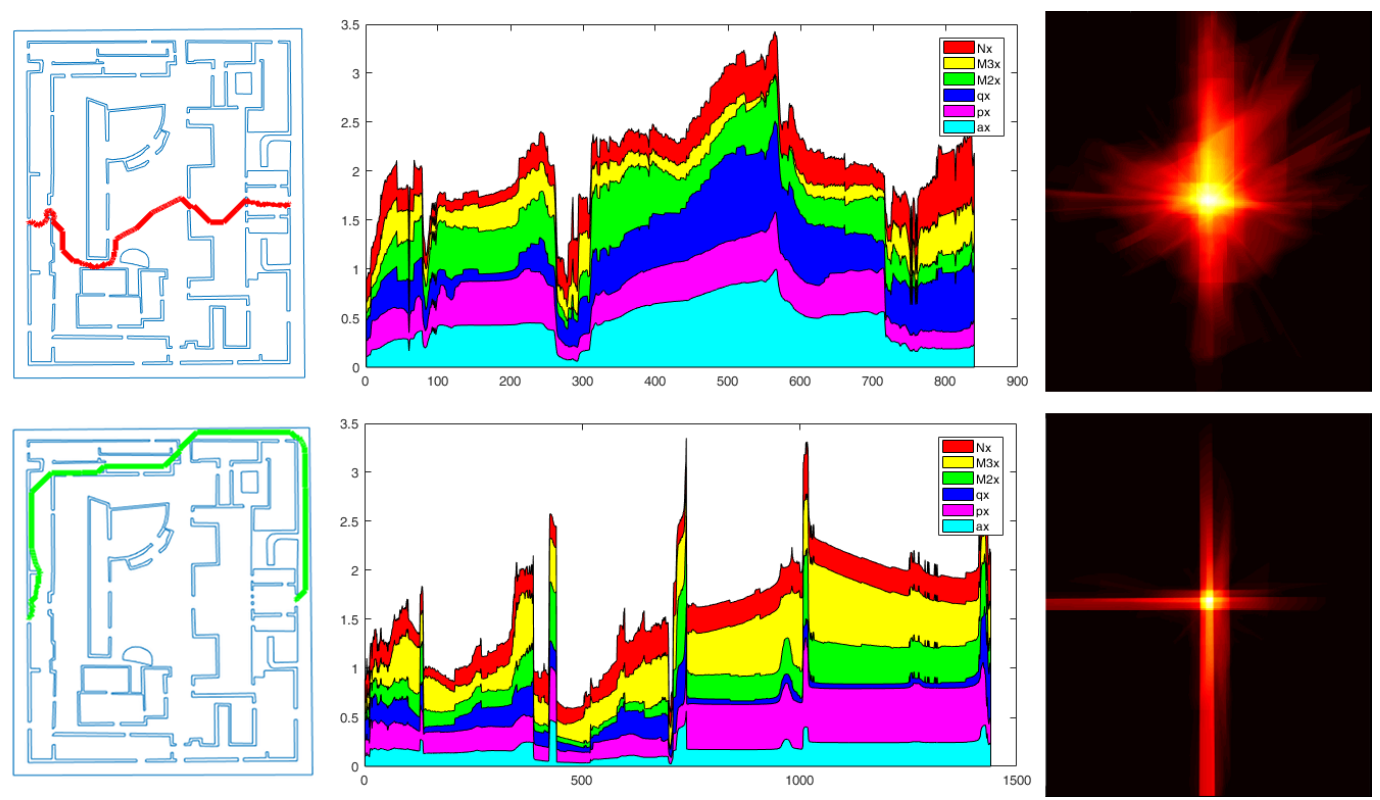

Abbildung 5.23: Drei Möglichkeiten zur Darstellung der realisierten archetypischen Routen für $k=2$ (siehe auch Abbildung 5.21a): Visualisierung im Gebäudeplan (links), gestapeltes Flächendiagramm der Isovisten-Messgrößen über die Zeit (Mitte) und gestapelte Isovisten (rechts).

um einen grundsätzlichen und einfacher zu erfassenden Eindruck einer Route zu ermöglichen. Im vorliegenden Beispiel sind eindeutig Musterveränderungen ersichtlich: die Form eines schmalen Kreuzes, die sich für die grüne Route beobachten lässt, impliziert, dass sich die zugrunde liegende Route in engen und rechtwinklig verlaufenden Gängen oder Straßen bewegt. Die recht unregelmäßige, großflächige Form in Abbildung 5.23 oben rechts impliziert eine Route, die durch weitläufige, abwechslungsreiche Bereiche des Gebäudes führt. Die Ergebnisse unterstreichen nochmals die These, dass unter der Einbeziehung der lokalen Umgebung mittels Isovisten-Messgrößen Routen gefunden werden, die sich nicht nur aufgrund ihrer Geometrie unterscheiden, sondern zusätzlich unterschiedliche Eigenschaften bezüglich der Wahrnehmung des Raums besitzen.

\subsection{Zusammenfassung}

Es existiert ein starkes Wechselspiel zwischen Routen und Karten, das auf der Tatsache basiert, dass Routen nicht allein für sich stehen, sondern in einer Karte eingebettet sind. Im Verlauf der Arbeit konnte beobachtet werden, dass sich der Fokus der vorgestellten Ansätze kontinuierlich von den Routen weg bewegt hat und die zugrunde liegenden Karten stärker in den Vordergrund gerückt sind. In Kapitel 3 wurden alternative Routen definiert und berechnet. 
Auch wenn die Routen erst durch die Homotopie definiert sind, die durch die Karte implizit wird, lag der Fokus doch auf den Routen selbst. Anschließend wurden in Kapitel 4 die archetypische Distanz und archetypische Routen definiert, das heißt Pfade, die möglichst unterschiedlich durch eine Karte verlaufen. Die ursprünglich verwendeten Merkmale wurden basierend auf der Geometrie der Routen bestimmt. In dem vorliegenden Kapitel wurde schließlich die lokale Umgebung einer Route untersucht. Somit wird der Fokus auf die zugrunde liegende Karten gelegt und es werden entsprechend extrahierte Informationen für unterschiedliche Anwendungen verwendet.

Der erste Teil dieses Kapitels behandelte die grundsätzliche Einbeziehung der lokalen Umgebung in den Kontext der alternativen Routen in komplexen Umgebungen. Es wurden verschiedene Möglichkeiten zur quantitativen Analyse der Wahrnehmung von Raum vorgestellt und schließlich wurde das Konzept der Isovisten gewählt, um dieses in weitere Algorithmen zu integrieren (siehe die erste der vier in Abschnitt 5.1 beschriebenen Herausforderungen). Anschließend wurden sogenannte diskrete Isovisten definiert. Das heißt, es wurden exakte Isovisten mittels eines einfachen Raycasting-Ansatzes angenähert. Innerhalb der Evaluation wurde gezeigt, dass die angenäherten Messwerte zwar einen systematischen Fehler besitzen, sie jedoch stark mit den exakten Werten korrelieren. Diskrete Isovisten können demnach als eine ausreichend gute Annäherung verwendet werden (siehe zweite Herausforderung in Abschnitt 5.1).

Im zweiten Teil dieses Kapitels wurde eine Anwendung der diskreten Isovisten-Messgrößen vorgestellt, nämlich die Annotation von Routen (siehe dritte Herausforderung). Es wurde gezeigt, dass durch die Identifikation der signifikanten Änderungen der lokalen Umgebung mittels lokaler Extrema vielversprechende Ergebnisse erzielt werden können. Das automatisierte Erkennen von Stellen, die auf eine Tür, die Mitte eines Raumes oder einen langen, monotonen Flur hinweisen, können für die semantische Evaluation von Routen in Navigationsszenarien innerhalb von Gebäuden verwendet werden.

Im Bereich der Einbeziehung und der einfachen Annäherung der lokalen Umgebung sowie der effizienten Annotation von Routen sind zahlreiche zukünftige Arbeiten denkbar. Zuerst sollte untersucht werden, wie die zwei in der vorliegenden Arbeit nicht einbezogenen Isovisten-Messgrößen (real-surface perimeter und occlusivity) im Kontext von Belegungsrastern implementiert werden können. Grundsätzlich kann anstelle eines Raycasting-Ansatzes ein Shadowcasting verwendet werden. Außerdem ist die grundsätzliche Vorgehensweise der bereits in [75] veröffentlichten Approximation näher zu analysieren und es können weitere alternative Herangehensweisen für die Annäherung der Messgrößen beschrieben werden. Der Einfluss der Pixelgröße wurde in der vorliegenden Arbeit nicht behandelt und ebenso kann der Fokus auf die Gradienten anstatt auf die einzelnen Werte gelegt werden.

Eine weitere Idee ist die Einbeziehung sogenannter gerichteter oder zirkulärer Statistik [17], [117]. Vorläufige, nicht veröffentlichte Untersuchungen des Autors legen nahe, dass beispielsweise die mean resultant length des Vektors 
der zirkulären Daten Routen innerhalb eines Gebäudes in sinnvolle Segmente partitioniert. Auch die circular range, also der kürzeste Kreisbogen, der den gesamten Datensatz enthält, scheint für die Identifizierung von durchlaufenen Kurven verwendbar zu sein.

Schließlich sollten die exakten, diskreten oder auch anderweitige IsovistenMessgrößen als Eingabe für anspruchsvollere Techniken innerhalb des Information Retrievals und des Representation Learnings verwendet werden. Beispielsweise kann unüberwachtes, überwachtes oder auch bestärkendes Lernen dazu eingesetzt werden, bestimmte Bereiche innerhalb einer Karte wie etwa Türen oder Kreuzungen zu finden. Ein weiteres Beispiel ist das Erlernen von bestimmten Bewegungsabläufen wie gelaufene Kurven. Solche Informationen können anschließend dazu verwendet werden, von Eigenschaften der Routen auf Eigenschaften der Karte zu schließen.

Im dritten Teil dieses Kapitels wurde der Frage nachgegangen, ob mittels der archetypischen Analyse von modellierten Merkmalen der räumlichen Wahrnehmung Erkenntnisse über die Umgebung erlangt werden können. Konkret wurde ein System vorgestellt, mit dem komplexe Umgebungen in Form von zweidimensionalen Karten hinsichtlich fundamentaler Unterschiede bezüglich der Sichtbarkeit segmentiert werden können. Dies bezieht sich auf die vierte in Abschnitt 5.1 beschriebene Herausforderung. Darüber hinaus wurde untersucht, welchen Einfluss Isovisten-Messgrößen bei der Berechnung archetypischer alternativer Routen besitzen. Das System ist unter Einbeziehung der Wahrnehmungseigenschaften einer Umgebung in der Lage, geeignete alternative Routen durch ein Gebäude zu erstellen, die unterschiedliche Benutzererfahrungen beim Durchlaufen des Gebäudes ermöglichen. Die Routen unterscheiden sich beispielsweise dadurch, dass sie ausschließlich enge und gerade Gänge durchlaufen oder aber offene und weite Hallen.

In zukünftigen Arbeiten sollen weitere Isovisten-Messgrößen oder auch andere Techniken zur quantitativen Analyse der Wahrnehmung von Raum zur Erstellung archetypischer Regionen und Routen verwendet und untersucht werden. Insbesondere der Einfluss einzelner Messgrößen soll stärker herausgearbeitet werden. Darüber hinaus können die archetypischen Regionen im Sinne des fuzzy clusterings mit einer Farbmischung dargestellt werden anstatt einer harten Zuordnung. Dies könnte eine feinere Interpretation der Berechnungsergebnisse zulassen.

Schließlich soll die in aller Kürze vorgestellte visuelle Analyse der Messgrößen strukturierter behandelt werden. Die Darstellung der gestapelten IsovistenMessgrößen sollten über die Zeit und insbesondere die gestapelten Isovisten mittels Visual Analytics zum Zwecke weiterer Informationsextraktion untersucht werden. 


\section{Zusammenfassung und Ausblick}

Spätestens mit dem Einbau von kostengünstigen GPS-Empfängern in Mobiltelefone und später Smartphones wurden ortsbezogene Anwendungen und Dienste für Millionen von Menschen ermöglicht [131]. Ebenso hat der technische Fortschritt bei der Reduzierung der physischen Größe von Prozessoren, Speicher und Konnektivität dabei geholfen, solche Dienste zusätzlich auf kleineren Geräten wie etwa Wearables verfügbar zu machen. Die Bedeutung einzelner Recheneinheiten schwindet, und es ergibt sich ein Internet der Dinge.

Navigation ist ein zentrales Thema solcher ortsbezogenen Anwendungen und Dienste, sowohl in der Anwendung als auch in der Forschung [87]-[89], [128], [129]. Die Anwendungsfälle sind dabei vielfältig und beinhalten das Finden interessanter Orte (Point of Interest), Geocaching oder das Anzeigen von Navigationsanweisungen im Straßenverkehr. In den letzten Jahren wurde zunehmend auch die Wegefindung in planmäßigen Netzen (öffentlicher Personennahverkehr wie Bus oder Bahn) und die Multimodalität (Kombination von Fortbewegungsmitteln wie zu Fuß, PKW oder Fahrrad) behandelt [11], [47], [50].

Eine Erweiterung des klassischen Wegfinde-Problems ist die Identifizierung von alternativen Routen, wobei dem Anwender mehrere Routen zur Auswahl gegeben werden. Dies ist gewissermaßen ein personalisiertes Routing, da der Anwender basierend auf Präferenzen $\left(\mathrm{CO}_{2}\right.$-Verbrauch, Mautgebühren, Spritverbrauch) oder Erfahrungen (landschaftlicher Wert, Risiko von Verkehrsstaus) wählen kann. Dieses Thema wurde bereits ausführlich in Straßennetzen behandelt, siehe beispielsweise die Übersicht von Bast et al. [11].

Das Berechnen und Verarbeiten von Routen wird jedoch ungleich schwieriger, wenn das relativ eingeschränkte Straßennetz verlassen wird und komplexe Umgebungen, also das freie Bewegen in Umgebungen, behandelt werden. Ein Fußgänger innerhalb eines Gebäudes kann sich nahezu in jede Richtung bewegen und Hindernisse umlaufen. Beispiele für Szenarien mit einem hohen Grad an Bewegungsfreiheit sind öffentliche Parks, aber auch große und komplexe Gebäude wie Krankenhäuser [110], Flughäfen [159] oder Messen.

Alternative Routen kommen nicht nur in Straßennetzen zur Anwendung, sondern auch in komplexen Umgebungen. Potentielle Anwender sind beispielsweise Besucher eines Flughafens mit unterschiedlich viel Zeit bis zum Boarding, Einsatzkräfte der Feuerwehr oder der Polizei, die blockierten Flächen ausweichen müssen, autonome mobile Roboter [172] in Lagerhäusern, die Pa- 
kete sammeln und lagern, und Nicht-Spieler-Charaktere in Computerspielen, die auf sinnvolle und gleichzeitig unvorhersehbare Art und Weise agieren [187]. Darüber hinaus kann ein Navigationssystem durch das Präsentieren von alternativen Routen Anwender dazu bringen, einen bereits stark frequentierten Bereich zu umgehen und Überfüllungen proaktiv zu vermeiden.

Das Problem der Routenplanung in Straßennetzen wird üblicherweise über die Abbildung des Straßennetzes auf einen Graphen mit Knoten und Kanten bewältigt, wodurch das Kürzester-Pfad-Problem effizient gelöst werden kann. In komplexen Umgebungen ist die große Herausforderung, eine angemessene Kartendarstellung zu finden, die einen guten Kompromiss zwischen der Einfachheit der Karte und deren Aussagekraft bietet. Je einfacher eine Kartendarstellung ist, desto schneller können Algorithmen darauf ausgeführt werden. Es existieren zahlreiche Ansätze, wie aus komplexen Umgebungen einfache Karten erstellt werden können. Eine Möglichkeit zur Darstellung von räumlichen Daten wie Gebäudeplänen sind Belegungsraster [139], bei denen Zellen entweder Hindernisse oder Freiflächen repräsentieren. Belegungsraster werden auch im Umfeld mobiler Roboter [63] oder unter Wasser [20] verwendet. Belegungsraster können zwar durch die zur Erstellung verwendeten Mapping-Algorithmen sowie fehlerbehaftete Sensoren ungenau sein [173], jedoch existieren viele Vorteile hinsichtlich der Effizienz bestimmter Operationen.

Die beschriebenen Gedanken stellen die Ausgangslage der vorliegenden Arbeit dar. Sie behandeln Ansätze, die alternative Routen in komplexen Umgebungen definieren, berechnen, vergleichen und für weitere Analysen verwenden. In den folgenden Abschnitten werden die Ergebnisse der vorliegenden Arbeit zusammengefasst und es wird ein Ausblick auf künftige Forschungsfragen gegeben.

\subsection{Zusammenfassung}

Der erste Teil der vorliegenden Arbeit hat die Berechnung alternativer Routen in Freiflächen behandelt. Dazu wurde die Verwendung des topologischen Konzepts der Homotopie vorgeschlagen, um zu entscheiden, ob zwei Routen als äquivalent oder alternativ anzusehen sind. Die Relation der Homotopie identifiziert Äquivalenzklassen, sodass ein Repräsentant einer Äquivalenzklasse eine Alternative bezüglich einer anderen Äquivalenzklasse ist. Diese Relation wird mithilfe der Fläche angenähert, die von den zwei Routen umschlossen wird. Ist ein Hindernis in dieser Fläche enthalten, so sind die Routen nichthomotop zueinander und werden als alternative Routen angesehen. In dieser Arbeit wurden zwei Algorithmen vorgestellt, die Homotopie-basiert alternative Routen in komplexen Umgebungen berechnen. Die Algorithmen arbeiten auf Belegungsraster, die aus unterschiedlichen Umgebungsmodellen erstellt werden können und eine effiziente Berechnung der Homotopie-Relation erlauben. Der One-Patching-Algorithmus basiert auf der Verwendung eines zusätzlichen Stützpunktes. Der Penalty-Algorithmus generiert alternative Routen, indem 
sukzessive der kürzeste Pfad teurer gemacht wird. Die Definition, die Annäherung der Relation sowie die Algorithmen zur Berechnung alternativer Routen wurden in unterschiedlichen Szenarien evaluiert. Darüber hinaus wurden bestehende Qualitätsmetriken zur Bewertung von alternativen Routen und Alternativgraphen in Straßennetzen strukturiert vorgestellt. Darauf basierend wurde diskutiert, warum diese Ansätze nicht unverändert im Umfeld komplexer Umgebungen eingesetzt werden können. Ziel der Diskussion war es, die Entwicklung von Qualitätsmetriken für alternative Routen und Alternativgraphen in komplexen Umgebungen anzuregen.

Der zweite Teil der vorliegenden Arbeit hat Möglichkeiten des Vergleichs von geospatialen Trajektorien behandelt. Die Überlastungswahrscheinlichkeit wurde eingeführt, um Routen innerhalb von Gebäuden zu sortieren und anschließend gezielt auszuwählen. Ausgehend von einem gegebenen Anwendungsfall können damit Routen gewählt werden, die entweder potentiell verkehrsreiche Flächen verwenden oder diese eher vermeiden. Die Idee dahinter basiert auf der Annahme, dass Punkte auf einer Karte, die häufig auf einem kürzesten Pfad liegen, auch häufig durchlaufen werden. Die Funktionsweise des entsprechenden Algorithmus wurde in unterschiedlichen Gebäudeplänen evaluiert. Des Weiteren wurde im zweiten Teil der vorliegenden Arbeit ein System vorgestellt, um eine Menge von Routen mit Hilfe der archetypischen Analyse innerhalb eines berechneten Merkmalsraums zu strukturieren. Die Ergebnisse zeigen, dass eine große Menge von Routen, die zwischen einem Start- und Zielpunkt verlaufen, in eine kleine Anzahl an offensichtlich unterschiedlichen Routen komprimiert werden kann. Das System erlaubt den Vergleich von Routen mit den ermittelten archetypischen Routen und auch mit sich selbst. Dieser Vergleich deckt nicht nur geometrische Ähnlichkeiten auf, sondern stellt vielmehr ein Maß für die Ähnlichkeit von Routen dar, das auf deren inhärenten Eigenschaften basiert.

Im dritten Teil der vorliegenden Arbeit wurde die Identifizierung von Strukturen in Gebäuden behandelt. Die grundsätzliche Idee dabei ist die Einbeziehung sogenannter Isovisten als numerische Repräsentation der lokalen Umgebung. Isovisten-Messgrößen bilden die menschliche Wahrnehmung von Raum demnach quantitativ ab. Es wurden diskrete Isovisten eingeführt, die mittels eines Raycasting-Ansatzes berechnet werden und als effiziente Annäherung der ursprünglichen Definition dienen. Die Messgrößen dieser diskreten Isovisten wurden zur Annotation von Routen innerhalb von Gebäuden verwendet. Darüber hinaus wurde das Clustering von Teilen eines Gebäudeplans mittels Isovisten-Messgrößen vorgestellt. Basierend auf den berechneten Messgrößen konnten sowohl alternative Routen, als auch archetypische Regionen innerhalb von Gebäuden abgeleitet werden. Die Idee hinter archetypischen Regionen ist es, genauere Erkenntnisse darüber zu erlangen, wie Teile von bestimmten Umgebungen in Gebäuden von Menschen empfunden werden. Dieser Ansatz soll helfen, eine Verbindung zwischen der subjektiven menschlichen Empfindung und den funktionalen Umgebungen in Gebäuden zu finden. 


\subsection{Ausblick}

Der topologische Begriff der Homotopie wurde in Abschnitt 3.2 zur Definition von alternativen Routen in komplexen Umgebungen verwendet. Je nach Szenario müssen sehr viele Routen auf Homotopie getestet werden. Daher wäre eine Methode wünschenswert, die in der Lage ist, eine große Menge von Routen gleichzeitig effizient zu testen. Darüber hinaus ist die Einbeziehung von im Vorfeld und unabhängig vom eigentlichen Verfahren erhobenen Informationen über die mögliche Anzahl an unterschiedlichen Homotopieklassen denkbar. So gibt es in einem Raum mit einer Säule in der Mitte zwischen zwei beliebigen Punkten exakt zwei nicht-homotope Routen ohne Selbstschnitt: links oder rechts um die Säule herum. In [118] wird ein solches Verfahren zur Aufzählung von Homotopieklassen vorgestellt. Darauf basierend kann zudem untersucht werden, ob die Länge einer Route oder die „Unterschiedlichkeit“ bezüglich einer Referenzroute ausschließlich basierend auf ihrer Homotopieklasse abgeschätzt werden kann, also ohne eigentliche Realisierung.

Die Heuristiken zum Finden nicht-homotoper Pfade (Abschnitt 3.3) können auf unterschiedliche Art erweitert werden. Die Integration der behandelten Qualitätsmetriken von alternativen Routen in die Berechnung derselben, die Erweiterung des One-Patching-Algorithmus in einen $k$-PatchingAlgorithmus sowie eine intelligentere Erhöhung der Kantengewichte beim Penalty-Algorithmus sind Beispiele dafür. Darüber hinaus können unterschiedliche Bewegungsmodelle [104] oder auch dynamische Veränderungen des Navigationsgraphen bei der Berechnung berücksichtigt werden. Auch die Verwendung von Metaheuristiken wie etwa Ant Colony Optimization [58] oder evolutionären Algorithmen im Allgemeinen [8] innerhalb des Berechnungsprozesses der Routen ist denkbar. Schließlich können weitere physische oder kinematische Einschränkungen beim Finden der nicht-homotopen Routen einbezogen werden. Beispiele dafür sind mobile Roboter mit definiertem Bewegungsvolumen oder solche, die durch einen starren Lenkvorgang nur einen bestimmten Wendekreis besitzen [79], [132].

Ein weiterer Themenkomplex für zukünftige Arbeiten ist die Nachbearbeitung der ermittelten alternativen Routen. Naheliegend ist eine Kurvenglättung, welche den charakteristischen Verlauf der Route beibehält, also mindestens die Homotopieklasse berücksichtigt. Es muss untersucht werden, ob und wie die bestehenden Ansätze zur Berechnung des kürzesten homotopen Pfades [18], [38], [46], [61], [105] in die Verfahren der vorliegenden Arbeit integriert werden können. Eine weitere Idee neben der Vereinfachung der Form der Routen ist die Kompression. Ein möglicher Ansatz wäre die Verwendung der topologischen Persistenz, sodass ,wichtige“ Punkte enthalten bleiben [60], [120]. Schließlich ist eine Segmentierung von alternativen Routen in komplexen Umgebungen mittels einer Art „partieller Homotopie“ denkbar. Über die gesamten Längen der zu untersuchenden Routen wird ein kontinuierlicher Wert der Homotopie berechnet, sodass ausgedrückt werden kann, „wie sehr“ zwei 
Routen homotop zueinander sind und zusammen mit einem Schwellwert „für wie lang“. Diese Idee ist ähnlich zur Homotopic Fréchet Distance [27].

In Abschnitt 3.5 wurden grundsätzliche Ideen für die Übertragbarkeit der Qualitätsmetriken für alternative Routen und Alternativgraphen von Straßennetzen in komplexe Umgebungen vorgestellt. Diese Ideen können weitergeführt und umgesetzt werden. Beispielsweise könnte mittels eines eindeutigen Verständnisses der Überlappung von zwei Routen der im Kontext von Straßennetzen existierende Plateau-Algorithmus [26] auch für die Verwendung in komplexen Umgebungen implementiert werden. In vielen Fällen hat sich gezeigt, dass die Übertragbarkeit der Qualitätsmetriken oft einen intelligenten Umgang mit verschiedenen Kartenrepräsentationen erfordert. Dies wurde vom Autor der vorliegenden Arbeit auch bei der Entwicklung einer Forschungsagenda für ortsbezogene Anwendungen und Dienste eingebracht, die im Kontext der ICA Commission on Location Based Services entsteht [112].

Es bestehen zahlreiche Möglichkeiten, das im Abschnitt 4.4.3 vorgestellte System zur Berechnung archetypischer Routen zu erweitern, sodass beispielsweise der Einfluss der verwendeten Merkmale zur Erstellung passenderer Kombinationen weiter analysiert werden kann. Zudem könnte die Verwendung alternativer Kartendarstellungen neben dem Belegungsraster ermöglicht werden sowie die möglichst automatisierte Wahl der Anzahl zu berechnenden Archetypen $k$ (zum Beispiel anhand der Koeffizientenmatrix $\alpha$ und der Doppelung von Homotopieklassen). Ebenso sollte die Verwendung von Erweiterungen der archetypischen Analyse wie etwa hierarchische, gewichtete, robuste oder evolutionäre Archetypen [68] im Kontext von alternativen Routen untersucht werden. Schließlich kann die Funktionsweise der vorgestellten archetypischen Distanz in zusätzlichen Anwendungsszenarien evaluiert und mit weiteren bestehenden Ansätzen zum Vergleich geospatialer Trajektorien wie etwa [180], [186] untersucht werden.

Die in Abschnitt 5.3 vorgestellte Definition diskreter Isovisten als Approximation der lokalen Umgebung wurde mittels eines Raycasting-Ansatzes durchgeführt, wodurch vier Isovisten-Messgrößen angenähert wurden. Weitere Arbeiten in diesem Bereich können für die Annäherung der zwei nicht definierten Messgrößen (real-surface perimeter und occlusivity) beispielsweise einen Shadowcasting-Ansatz oder grundsätzlich andere Vorgehensweisen der Annäherung mittels Strahlen verwenden. Ebenso ist eine Einbeziehung der Messgrößen benachbarter Punkte im Raum als Isovisten-Felder denkbar. Die vorgestellte Annotation von Routen hat viel Potential für weiterführende Forschungsarbeiten gezeigt. Eine Idee wäre die Verwendung der Bayesian Surprise [115], [153], einer mathematischen Beschreibung von menschlicher Überraschung. Eine gegebene Route könnte demnach annotiert oder segmentiert werden, wenn sich die numerische Beschreibung der lokalen Umgebung unerwartet ändert.

Schließlich gilt es, die unter Verwendung der lokalen Umgebung erstellten archetypischen alternativen Routen sowie die archetypischen Regionen (Abschnitt 5.4) mit Techniken des maschinellen Lernens zu untersuchen. Die 
Isovisten-Messgrößen scheinen passende Repräsentationen der räumlichen Umgebung zu sein, um beispielsweise die Darstellung von Türdurchgängen mittels künstlicher neuronaler Netze [16] zu erlernen. Eine weitere Idee ist die gezielte Synthese von Routen oder Regionen mit bestimmten Eigenschaften. Beispiele für Anweisungen an ein solches System können ,erstelle mir eine Route, bei der ich schwer beobachtet werden kann“ oder „erstelle mir einen Gebäudeplan, der viele vertikale Gänge besitzt" sein. 


\section{Literaturverzeichnis}

[1] I. Abraham, D. Delling, A. V. Goldberg und R. F. Werneck, „Alternative routes in road networks", in International Symposium on Experimental Algorithms (SEA). Lecture Notes in Computer Science 6049, Springer, 2010, S. 23-34.

[2] I. Abraham, D. Delling, A. V. Goldberg und R. F. Werneck, „Alternative routes in road networks", Journal of Experimental Algorithmics (JEA), Jg. 18, S. 1-3, 2013.

[3] R. Agrawal, C. Faloutsos und A. Swami, ,Efficient similarity search in sequence databases", Foundations of data organization and algorithms, S. 69-84, 1993.

[4] L. Almeida, J. Ji, G. Steinbauer und S. Luke, RoboCup 2015: Robot World Cup XIX. Springer, 2016, Bd. 9513.

[5] M. Ankerst, M. M. Breunig, H.-P. Kriegel und J. Sander, „OPTICS: ordering points to identify the clustering structure", in ACM Sigmod record, ACM, Bd. 28, 1999, S. 49-60.

[6] M. A. Armstrong, Basic topology. Springer, 1983.

[7] J. Arz, D. Luxen und P. Sanders, ,Transit node routing reconsidered“, in International Symposium on Experimental Algorithms, Springer, 2013, S. 55-66.

[8] T. Back, Evolutionary algorithms in theory and practice: evolution strategies, evolutionary programming, genetic algorithms. Oxford university press, 1996.

[9] K. Backhaus, B. Erichson, W. Plinke und R. Weiber, Multivariate Analysemethoden: eine anwendungsorientierte Einführung. Springer-Verlag, 2015.

[10] R. Bader, J. Dees, R. Geisberger und P. Sanders, „Alternative route graphs in road networks", in Theory and Practice of Algorithms in (Computer) Systems, Springer, 2011, S. 21-32.

[11] H. Bast, D. Delling, A. Goldberg, M. Müller-Hannemann, T. Pajor, P. Sanders, D. Wagner und R. F. Werneck, „Route planning in transportation networks“, in Algorithm Engineering, Springer, 2016, S. 19-80.

[12] M. Batty, „Exploring isovist fields: space and shape in architectural and urban morphology", Environment and planning B: Planning and Design, Jg. 28, Nr. 1, S. 123-150, 2001. 
[13] R. Bauer, D. Delling, P. Sanders, D. Schieferdecker, D. Schultes und D. Wagner, „Combining hierarchical and goal-directed speed-up techniques for dijkstra's algorithm“, Journal of Experimental Algorithmics (JEA), Jg. 15, S. 1-31, 2010.

[14] N. Beckmann, H.-P. Kriegel, R. Schneider und B. Seeger, ,The R*-tree: An Efficient and Robust Access Method for Points and Rectangles", in ACM SIGMOD Record, ACM, Bd. 19, 1990, S. 322-331.

[15] M. L. Benedikt, "To take hold of space: isovists and isovist fields", Environment and Planning B: Planning and design, Jg. 6, Nr. 1, S. 4765, 1979.

[16] Y. Bengio, A. Courville und P. Vincent, „Representation learning: A review and new perspectives", IEEE Transactions on Pattern Analysis and Machine Intelligence, Jg. 35, Nr. 8, S. 1798-1828, 2013.

[17] P. Berens, „CircStat: a MATLAB toolbox for circular statistics“, Journal of Statistical Software, Jg. 31, Nr. 10, S. 1-21, 2009.

[18] S. Bespamyatnikh, „Computing homotopic shortest paths in the plane", in Proceedings of the 14th annual ACM-SIAM Symposium on Discrete algorithms, Society for Industrial und Applied Mathematics, 2003, S. 609-617.

[19] G. Best, „Direction-finding in large buildings“, Architectural Psychology. London: RIBA Publications, S. 72-75, 1970.

[20] F. Bonin-Font, A. Ortiz und G. Oliver, ,Visual Navigation for Mobile Robots: A Survey", Journal of Intelligent and Robotic Systems, Jg. 53, Nr. 3, S. 263-296, 2008.

[21] G. E. Bredon, Topology and Geometry. Springer, 1993, Bd. 139.

[22] J. E. Bresenham, „Algorithm for Computer Control of a Digital Plotter“, IBM Systems Journal, Jg. 4, Nr. 1, S. 25-30, 1965.

[23] BuildingGreen, We Spend 90\% of Our Time Indoors. Says Who?, 2016. Adresse: https: //www .buildinggreen.com/blog/we-spend-90-ourtime-indoors-says-who.

[24] Bundesministerium für Bildung und Forschung (BMBF), Die neue Hightech-Strategie: Innovationen für Deutschland, 2014. Adresse: https : //www.bmbf.de/pub_hts/HTS_Broschure_Web.pdf.

[25] Bundesministerium für Bildung und Forschung (BMBF), Fortschritt durch Forschung und Innovation: Bericht zur Umsetzung der HightechStrategie, 2017. Adresse: https : //www . bmbf .de/pub/Fortschritt_ durch_Forschung_und_Innovation.pdf.

[26] Cambridge Vehicle Information Technology Ltd., Choice Routing, 2013. Adresse: http : / / www . camvit . com / camvit-technical-english / Camvit-Choice-Routing-Explanation-english.pdf. 
[27] E. W. Chambers, E. C. De Verdiere, J. Erickson, S. Lazard, F. Lazarus und S. Thite, „Homotopic Fréchet distance between curves or, walking your dog in the woods in polynomial time", Computational Geometry, Jg. 43, Nr. 3, S. 295-311, 2010.

[28] G. Chen und D. Kotz, „Asurvey of context-aware mobile computing research“, Technical Report TR2000-381, Dept. of Computer Science, Dartmouth College, Techn. Ber., 2000.

[29] L. Chen, M. T. Özsu und V. Oria, ,,Robust and fast similarity search for moving object trajectories", in Proceedings of the 2005 ACM SIGMOD International Conference on Management of Data, ACM, 2005, S. 491502.

[30] Y. Chen, M. G. Bell und K. Bogenberger, „Reliable pretrip multipath planning and dynamic adaptation for a centralized road navigation system", IEEE Transactions on Intelligent Transportation Systems, Jg. 8, Nr. 1, S. 14-20, 2007.

[31] Communications of the ACM, Contributed Articles: Spatial Computing, 2016. Adresse: https : / cacm. acm.org/magazines/2016/1/195727spatial-computing/abstract.

[32] R. Conroy-Dalton, ,Spatial navigation in immersive virtual environments", Diss., University of London, 2001.

[33] T. H. Cormen, Introduction to Algorithms. MIT press, 2009.

[34] A. Corral, Y. Manolopoulos, Y. Theodoridis und M. Vassilakopoulos, „Closest pair queries in spatial databases", in ACM SIGMOD Record, ACM, Bd. 29, 2000, S. 189-200.

[35] G. F. Coulouris, J. Dollimore und T. Kindberg, Distributed Systems: Concepts and Design. Pearson Education, 2005.

[36] A. Cutler und L. Breiman, „Archetypal analysis“, Technometrics, Jg. 36, Nr. 4, S. 338-347, 1994.

[37] C. P. Dancey und J. Reidy, Statistics without maths for psychology. Pearson Education, 2007.

[38] S. Daneshpajouh und M. Ghodsi, „Aheuristic homotopic path simplification algorithm", in International Conference on Computational Science and Its Applications, Springer, 2011, S. 132-140.

[39] M. Daszykowski, B. Walczak und D. Massart, „Looking for natural patterns in data: Part 1. Density-based approach", Chemometrics and Intelligent Laboratory Systems, Jg. 56, Nr. 2, S. 83-92, 2001.

[40] C. Davies, R. Mora und D. Peebles, „Isovists for orientation: Can space syntax help us predict directional confusion", in Proceedings of the Space Syntax and Spatial Cognition Workshop, Spatial Cognition, 2006. 
[41] L. S. Davis und M. L. Benedikt, „Computational models of space: Isovists and isovist fields", Computer graphics and image processing, Jg. 11, Nr. 1, S. 49-72, 1979.

[42] J. De Leeuw und P. Mair, ,Multidimensional scaling using majorization: SMACOF in R", Department of Statistics, UCLA, 2011.

[43] M. J. De Smith, M. F. Goodchild und P. Longley, Geospatial analysis: a comprehensive guide to principles, techniques and software tools. Troubador Publishing Ltd, 2007.

[44] J. Dees, „Computing Alternative Routes in Road Networks“, Magisterarb., Karlsruhe Institut für Technologie, Fakultät für Informatik, 2010.

[45] J. Dees, R. Geisberger, P. Sanders und R. Bader, „Defining and computing alternative routes in road networks", arXiv preprint arXiv:1002.4330, 2010.

[46] L. Deleuran, „Homotopic Polygonal Line: Simplification“, Diss., Department Office Computer Science, Aarhus University, 2012.

[47] D. Delling, J. Dibbelt, T. Pajor, D. Wagner und R. F. Werneck, „Computing Multimodal Journeys in Practice", in Proceedings of the 12th International Symposium on Experimental Algorithms (SEA'13), Springer, 2013, S. 260-271.

[48] D. Delling, A. V. Goldberg, T. Pajor und R. F. Werneck, „Customizable route planning", in International Symposium on Experimental Algorithms (SEA). Lecture Notes in Computer Science 6630, Springer, 2011, S. 376-387.

[49] D. Delling, A. V. Goldberg, T. Pajor und R. F. Werneck, „Customizable route planning in road networks", Transportation Science, 2015.

[50] D. Delling, B. Katz und T. Pajor, „Parallel computation of best connections in public transportation networks", Journal of Experimental Algorithmics (JEA), Jg. 17, S. 4.1-4.26, 2012.

[51] D. Delling und D. Wagner, „Pareto paths with SHARC“, in International Symposium on Experimental Algorithms (SEA), Springer, 2009, S. $125-136$.

[52] D. Delling und R. F. Werneck, „Faster customization of road networks“, in International Symposium on Experimental Algorithms (SEA). Lecture Notes in Computer Science 7933, Springer, 2013, S. 30-42.

[53] A. K. Dey und G. D. Abowd, „Towards a better understanding of context and context-awareness", in International Symposium on Handheld and Ubiquitous Computing, Springer, 1999, S. 304-307.

[54] Digital-Gipfel-Plattform Digitale Netze und Mobilität, Plattform "Digitale Netze und Mobilität": Wegweiser in die digitale Zukunft, 2017. Adresse: http : / / plattform-digitale-netze . de / app / uploads / 2017/06/PF1-Hauptdokument2017.pdf. 
[55] Digital-Gipfel-Plattform Digitale Netze und Mobilität, Roadmap Intelligente Mobilität: Empfehlungen für einen Handlungsplan, 2017. Adresse: http://plattform-digitale-netze.de/app/uploads/2017/06/ Roadmap_Intelligente_Mobilitaet.pdf.

[56] E. W. Dijkstra, "Anote on two problems in connexion with graphs“, Numerische mathematik, Jg. 1, Nr. 1, S. 269-271, 1959.

[57] U. Dogu und F. Erkip, ,,Spatial Factors Affecting Wayfinding and Orientation: A Case Study in a Shopping Mall“", Environment and Behavior, Jg. 32, Nr. 6, S. 731-755, 2000.

[58] M. Dorigo und G. Di Caro, „Ant colony optimization: a new metaheuristic", in Proceedings of the 1999 Congress on Evolutionary Computation (CEC), IEEE, Bd. 2, 1999, S. 1470-1477.

[59] B. Eberhardinger, H. Seebach, A. Knapp und W. Reif, „Towards testing self-organizing, adaptive systems", in IFIP International Conference on Testing Software and Systems, Springer, 2014, S. 180-185.

[60] H. Edelsbrunner, D. Letscher und A. Zomorodian, ,Topological persistence and simplification", in Proceedings of the 41st Annual Symposium on Foundations of Computer Science, IEEE, 2000, S. 454-463.

[61] A. Efrat, S. G. Kobourov und A. Lubiw, „Computing homotopic shortest paths efficiently“, Computational Geometry, Jg. 35, Nr. 3, S. 162$172,2006$.

[62] M. Ehrgott, Multicriteria optimization. Springer Science \& Business Media, 2006.

[63] A. Elfes, „Using Occupancy Grids for Mobile Robot Perception and Navigation", Computer, Jg. 22, Nr. 6, S. 46-57, 1989.

[64] B. Emo, „Exploring isovists : the egocentric perspective“, in International Space Syntax Symposium, 2015, S. 1-8.

[65] B. Emo, C. Hoelscher, J. Wiener und R. Dalton, „Wayfinding and spatial configuration: evidence from street corners", in International Space Syntax Symposium, 2012, S. 1-16.

[66] M. Ester, H.-P. Kriegel, J. Sander und X. Xu, „Adensity-based algorithm for discovering clusters in large spatial databases with noise", in Proceedings of the 2nd International Conference on Knowledge Discovery and Data Mining (KDD'96), 1996, S. 226-231.

[67] M. Ester und J. Sander, Knowledge discovery in databases: Techniken und Anwendungen. Springer-Verlag, 2013.

[68] M. J. A. Eugster, Archetypal Analysis: Mining the Extreme, HIIT Seminar, Helsinki Institute for Information Technology, 2012.

[69] M. J. A. Eugster, Archetypal Athletes, 2011. Adresse: https://epub. ub.uni-muenchen.de/12336/. 
[70] M. J. A. Eugster und F. Leisch, „From Spider-Man to Hero: Archetypal Analysis in R", Journal of Statistical Software, Jg. 30, Nr. 8, S. 1-23, 2009.

[71] M. J. A. Eugster und F. Leisch, ,Weighted and robust archetypal analysis", Computational Statistics \& Data Analysis, Jg. 55, Nr. 3, S. 1215$1225,2011$.

[72] European Road Transport Research Advisory Council (ERTRAC), Automated Driving Roadmap, 2015. Adresse: http: //www . ertrac .org/ uploads/documentsearch/id38/ERTRAC_Automated-Driving-2015. pdf.

[73] S. Feld, „Scoring of Alternative Routes Using Implicit Building Topologies", in Proceedings of the Science and Information Conference (SAI 2015), IEEE, 2015, S. 329-336.

[74] S. Feld, H. Lyu und A. Keler, ,Identifying Divergent Building Structures Using Fuzzy Clustering of Isovist Features", in Progress in LocationBased Services 2016, Proceedings of the 13th International Conference on Location-Based Services (ICA LBS 2016), Springer International Publishing, 2017, S. 151-172.

[75] S. Feld, M. Werner und C. Linnhoff-Popien, „Approximated Environment Features With Application to Trajectory Annotation", in Proceedings of the 6th IEEE Symposium Series on Computational Intelligence (IEEE SSCI 2016), IEEE, 2016, S. 1-7.

[76] S. Feld, M. Werner und C. Linnhoff-Popien, ,Criteria for Selecting Small Sets of Alternative Routes in Free Space Scenarios", in Proceedings of the 13th International Conference on Location-Based Services (ICA LBS 2016), 2016, S. 1-18.

[77] S. Feld, M. Werner, M. Schönfeld und S. Hasler, „Archetypes of Alternative Routes in Buildings", in Proceedings of the 6th International Conference on Indoor Positioning and Indoor Navigation (IPIN 2015), IEEE, 2015, S. 1-10.

[78] R. W. Floyd, „Algorithm 97: Shortest Path“, Communications of the ACM, Jg. 5, Nr. 6, S. 345-, 1962.

[79] T. Fraichard, „Smooth trajectory planning for a car in a structured world", in Proceedings of the 1991 IEEE International Conference on Robotics and Automation, IEEE, 1991, S. 318-323.

[80] G. Franz und J. M. Wiener, „From space syntax to space semantics: a behaviorally and perceptually oriented methodology for the efficient description of the geometry and topology of environments", Environment and Planning B: Planning and Design, Jg. 35, Nr. 4, S. 574-592, 2008. 
[81] M. M. Fréchet, „Sur quelques points du calcul fonctionnel“, Rendiconti del Circolo Matematico di Palermo (1884-1940), Jg. 22, Nr. 1, S. 1-72, 1906.

[82] M. L. Fredman und R. E. Tarjan, „Fibonacci heaps and their uses in improved network optimization algorithms", Journal of the ACM (JACM), Jg. 34, Nr. 3, S. 596-615, 1987.

[83] T. Gärling, „Studies in visual perception of architectural spaces and Rooms: I. Judgment scales of open and closed space", Scandinavian Journal of Psychology, Jg. 10, Nr. 1, S. 250-256, 1969.

[84] T. Gärling, „Studies in visual perception of architectural spaces and Rooms: II. Judgments of open and closed space by category rating and magnitude estimation", Scandinavian Journal of Psychology, Jg. 10, Nr. 1, S. 257-268, 1969.

[85] T. Gärling, „Studies in visual perception of architectural spaces and Rooms: III. A relation between judged depth and size of space", Scandinavian Journal of Psychology, Jg. 11, Nr. 1, S. 124-131, 1970.

[86] T. Gärling, „Studies in visual perception of architectural spaces and Rooms: IV. The relation of judged depth to jugded size of space under different viewing conditions", Scandinavian Journal of Psychology, Jg. 11, Nr. 1, S. 133-145, 1970.

[87] G. Gartner, W. Cartwright und M. P. Peterson, Hrsg., Location Based Services and TeleCartography. Springer Science \& Business Media, 2007.

[88] G. Gartner und F. Ortag, Hrsg., Advances in Location-Based Services. Springer, 2012.

[89] G. Gartner und K. Rehrl, Hrsg., Location Based Services and TeleCartography II : From Sensor Fusion to Context Models. Springer, 2009.

[90] R. Geisberger, P. Sanders, D. Schultes und D. Delling, „Contraction hierarchies: Faster and simpler hierarchical routing in road networks", in International Workshop on Experimental and Efficient Algorithms, Springer, 2008, S. 319-333.

[91] R. Geisberger, P. Sanders, D. Schultes und C. Vetter, „Exact routing in large road networks using contraction hierarchies“, Transportation Science, Jg. 46, Nr. 3, S. 388-404, 2012.

[92] J. J. Gibson, „The senses considered as perceptual systems.“, 1966.

[93] T. Giorgino, „Computing and visualizing dynamic time warping alignments in R: the dtw package“, Journal of Statistical Software, Jg. 31, Nr. 7, S. 1-24, 2009. 
[94] A. V. Goldberg und C. Harrelson, „Computing the shortest path: A search meets graph theory", in Proceedings of the 16th annual ACMSIAM symposium on Discrete algorithms, Society for Industrial und Applied Mathematics, 2005, S. 156-165.

[95] E. Guizzo, ,Three engineers, hundreds of robots, one warehouse“, IEEE spectrum, Jg. 45, Nr. 7, S. 26-34, 2008.

[96] E. Guizzo, „How google's self-driving car works“, IEEE Spectrum Online, October, Jg. 18, 2011.

[97] J. Han, J. Pei und M. Kamber, Data mining: Concepts and Techniques. Elsevier, 2011.

[98] S. Haq und C. Zimring, ,Just down the road a piece: the development of topological knowledge of building layouts", Environment and Behavior, Jg. 35, Nr. 1, S. 132-160, 2003.

[99] P. E. Hart, N. J. Nilsson und B. Raphael, „Aformal basis for the heuristic determination of minimum cost paths", IEEE Transactions on Systems Science and Cybernetics, Jg. 4, Nr. 2, S. 100-107, 1968.

[100] J. A. Hartigan, Clustering Algorithms. John Wiley \& Sons, Inc. New York, NY, USA, 1975.

[101] S. Hasler, „Archetypal Analysis in Indoor Navigation Scenarios“, Projektarbeit, Ludwig-Maximilians-Universität München, 2014.

[102] F. Hausdorff, Mengenlehre. Walter de Gruyter Berlin, 1927.

[103] S. C. Hayward und S. S. Franklin, „Perceived openness-enclosure of architectural space", Environment and Behavior, Jg. 6, Nr. 1, S. 37-52, 1974.

[104] D. Helbing, P. Molnár, I. J. Farkas und K. Bolay, „Self-organizing pedestrian movement", Environment and Planning B: Planning and Design, Jg. 28, Nr. 3, S. 361-383, 2001.

[105] J. Hershberger und J. Snoeyink, „Computing minimum length paths of a given homotopy class", Computational Geometry, Jg. 4, Nr. 2, S. 6397, 1994 .

[106] M. Hilger, E. Köhler, R. H. Möhring und H. Schilling, „Fast pointto-point shortest path computations with arc-flags", The Shortest Path Problem: Ninth DIMACS Implementation Challenge, Jg. 74, S. 41-72, 2009.

[107] B. Hillier und J. Hanson, The social logic of space. Cambridge university press, 1984.

[108] C. Hölscher, T. Meilinger, G. Vrachliotis, M. Brösamle und M. Knauff, „Finding the way inside: Linking architectural design analysis and cognitive processes", in Spatial Cognition IV. Reasoning, Action, Interaction, Springer, 2005, S. 1-23. 
[109] J. F. Hughes und J. D. Foley, Computer Graphics: Principles and Practice. Pearson Education, 2014.

[110] N. Hughes, J. Pinchin, M. Brown und D. Shaw, ,Navigating in large hospitals", in Proceedings of the 6th International Conference on Indoor Positioning and Indoor Navigation (IPIN 2015), IEEE, 2015, S. 1-9.

[111] R. Huitl, G. Schroth, S. Hilsenbeck, F. Schweiger und E. Steinbach, „TUMindoor: An extensive image and point cloud dataset for visual indoor localization and mapping", in Proceedings of the 19th IEEE International Conference on Image Processing (ICIP 2012), IEEE, 2012, S. 1773-1776.

[112] ICA Commission on Location-Based Services, LBS Research Agenda: Key Problems (Draft), 2016. Adresse: http://lbs .icaci .org/wpcontent/uploads/2016/05/Key_Problems.pdf.

[113] IEEE Spectrum, Another Robot to Enter Fukushima Reactor, and We Wish It Were Modular, http://spectrum.ieee.org/automaton/robotics/industrial-robots/hereswhy-we-should-be-using-modular-robots-to-explore-fukushima, 2015.

[114] R. Ihaka und R. Gentleman, „R: a language for data analysis and graphics", Journal of Computational and Graphical Statistics, Jg. 5, Nr. 3, S. 299-314, 1996.

[115] L. Itti und P. Baldi, „Bayesian surprise attracts human attention“, Vision Research, Jg. 49, Nr. 10, S. 1295-1306, 2009.

[116] A. K. Jain und R. C. Dubes, Algorithms for clustering data. PrenticeHall, Inc., 1988.

[117] S. R. Jammalamadaka und A. Sengupta, Topics in Circular Statistics. World Scientific, 2001, Bd. 5.

[118] K. D. Jenkins, ,The shortest path problem in the plane with obstacles: A graph modeling approach to producing finite search lists of homotopy classes“, Diss., Monterey, California. Naval Postgraduate School, 1991.

[119] R. Jütte, Geschichte der Sinne: von der Antike bis zum Cyberspace. CH Beck, 2000.

[120] P. Katsikouli, R. Sarkar und J. Gao, „Persistence based online signal and trajectory simplification for mobile devices", in Proceedings of the 22nd ACM SIGSPATIAL International Conference on Advances in Geographic Information Systems, ACM, 2014, S. 371-380.

[121] L. Kaufman und P. J. Rousseeuw, Finding Groups in Data: an Introduction to Cluster Analysis. John Wiley \& Sons, 2009, Bd. 344.

[122] H. Kitano, M. Asada, Y. Kuniyoshi, I. Noda und E. Osawa, „Robocup: The robot world cup initiative", in Proceedings of the 1st International Conference on Autonomous Agents, ACM, 1997, S. 340-347. 
[123] N. E. Klepeis, W. C. Nelson, W. R. Ott, J. P. Robinson, A. M. Tsang, P. Switzer, J. V. Behar, S. C. Hern und W. H. Engelmann, „The National Human Activity Pattern Survey (NHAPS): a resource for assessing exposure to environmental pollutants", Journal of Exposure Science and Environmental Epidemiology, Jg. 11, Nr. 3, S. 231, 2001.

[124] M. Kobitzsch, „An alternative approach to alternative routes: HiDAR“, in European Symposium on Algorithms, Springer, 2013, S. 613-624.

[125] M. Kobitzsch, M. Radermacher und D. Schieferdecker, „Evolution and evaluation of the penalty method for alternative graphs", in 13th Workshop on Algorithmic Approaches for Transportation Modelling, Optimization, and Systems (ATMOS), Schloss Dagstuhl - Leibniz-Zentrum für Informatik, 2013, S. 94-107.

[126] J. Krisp, L. Ding, Y. Jin und P. Peer, „Indoor Routing: Is a centrality measure for an indoor routing network useful?", in Mobile Tartu 2012, 2012 , S. $22-25$.

[127] J. Krisp, L. Liu und T. Berger, „Goal Directed Visibility Polygon Routing for Pedestrian Navigation", in International Symposium on LBS $\mathscr{6}$ Tele Cartography, 2010.

[128] J. M. Krisp, Hrsg., Progress in Location-Based Services. Springer, 2013.

[129] J. M. Krisp und L. Meng, „Going local: evolution of location based services", in Progress in Location-Based Services, Springer, 2013, S. 1115.

[130] J. M. Krisp, P. Peer und L. Ding, „Classification of an indoor routing network based on graph theory", in GeoInformatics 2012, 2012.

[131] A. Küpper, Location-Based Services: Fundamentals and Operation. John Wiley \& Sons, Ltd, 2005.

[132] J.-P. Laumond, ,Finding Collision-Free Smooth Trajectories for a NonHolonomic Mobile Robot", in Proceedings of the 10th International Joint Conferences on Artificial Intelligence (IJCAI 1987), Bd. 10, 1987, S. 1120-1123.

[133] V. I. Levenshtein, „Binary codes capable of correcting deletions, insertions, and reversals", in Soviet physics doklady, Bd. 10, 1966, S. 707710 .

[134] R. V. Levine und A. Norenzayan, ,The pace of life in 31 countries“, Journal of Cross-Cultural Psychology, Jg. 30, Nr. 2, S. 178-205, 1999.

[135] H. Liu, H. Darabi, P. Banerjee und J. Liu, „Survey of wireless indoor positioning techniques and systems", IEEE Transactions on Systems, Man, and Cybernetics, Part C (Applications and Reviews), Jg. 37, Nr. 6, S. 1067-1080, 2007. 
[136] D. Luxen und D. Schieferdecker, „Candidate sets for alternative routes in road networks", in International Conference on Experimental Algorithms, Springer-Verlag, 2012, S. 260-270.

[137] J. MacQueen, ,Some methods for classification and analysis of multivariate observations", in Proceedings of the 5th Berkeley Symposium on Mathematical Statistics and Probability, Oakland, CA, USA., Bd. 1, 1967, S. 281-297.

[138] R. S. Montero und E. Bribiesca, ,State of the art of compactness and circularity measures", in International Mathematical Forum, Bd. 4, 2009, S. $1305-1335$.

[139] H. Moravec und A. Elfes, „High Resolution Maps from Wide Angle Sonar", in Proceedings of the 1985 IEEE International Conference on Robotics and Automation, IEEE, Bd. 2, 1985, S. 116-121.

[140] J. R. Munkres, Topology: a First Course. Prentice-Hall Englewood Cliffs, NJ, 1975, Bd. 23.

[141] K. Nagatani, S. Kiribayashi, Y. Okada, K. Otake, K. Yoshida, S. Tadokoro, T. Nishimura, T. Yoshida, E. Koyanagi, M. Fukushima u. a., „Emergency response to the nuclear accident at the Fukushima Daiichi Nuclear Power Plants using mobile rescue robots", Journal of Field Robotics, Jg. 30, Nr. 1, S. 44-63, 2013.

[142] T. A. J. Nicholson, „Finding the shortest route between two points in a network“, The Computer Journal, Jg. 9, Nr. 3, S. 275-280, 1966.

[143] S. Nijhuis, „GIS-based Landscape Design Research: Exploring Aspects of Visibility in Landscape Architectonic Compositions", in Geodesign by Integrating Design and Geospatial Sciences, Springer, 2014, S. 193-217.

[144] Nokia, Nokia completes next stage of transformation with agreement to sell HERE to automotive industry consortium at an enterprise value of EUR 2.8 billion, 2015. Adresse: http://www.nokia.com/en_int/ news / releases / 2015/08/03/nokia - completes - next - stage - of transformation-with-agreement-to-sell-here-to-automotiveindustry - consortium - at - an - enterprise - value - of - eur - 28 billion.

[145] K. Ogawa, E. Verbree, S. Zlatanova, N. Kohtake und Y. Ohkami, „Toward seamless indoor-outdoor applications: Developing stakeholderoriented location-based services", Geo-spatial Information Science, Jg. 14, Nr. 2, S. 109-118, 2011.

[146] R. Osserman, „The isoperimetric inequality“, Bulletin of the American Mathematical Society, Jg. 84, Nr. 6, S. 1182-1238, 1978.

[147] A. Paraskevopoulos und C. Zaroliagis, „Improved Alternative Route Planning", in OASIcs-OpenAccess Series in Informatics, Schloss Dagstuhl - Leibniz-Zentrum für Informatik, Bd. 33, 2013. 
[148] J. Peponis, J. Wineman, M. Rashid, S. H. Kim und S. Bafna, „On the description of shape and spatial configuration inside buildings: convex partitions and their local properties", Environment and Planning B: planning and Design, Jg. 24, Nr. 5, S. 761-781, 1997.

[149] M. P. Peterson, Hrsg., Advances in Cartography and GIScience: Selections from the International Cartographic Conference 2017, Ser. Publications of the International Cartographic Association (ICA). Springer International Publishing, 2017.

[150] G. C. Porzio, G. Ragozini und D. Vistocco, „On the use of archetypes as benchmarks", Applied Stochastic Models in Business and Industry, Jg. 24, Nr. 5, S. 419-437, 2008.

[151] G. Pratt und J. Manzo, „The darpa robotics challenge“, IEEE Robotics \& Automation Magazine, Jg. 20, Nr. 2, S. 10-12, 2013.

[152] M. Radermacher, „Schnelle Berechnung von Alternativgraphen“, Bachelor's Thesis, Magisterarb., Karlsruhe Institut für Technologie, Fakultät für Informatik, 2012.

[153] A. Ranganathan und F. Dellaert, „Bayesian surprise and landmark detection", in Proceedings of the 2009 IEEE International Conference on Robotics and Automation (IEEE ICRA 2009), IEEE, 2009, S. 20172023.

[154] P. Rashidi und A. Mihailidis, „Asurvey on ambient-assisted living tools for older adults", IEEE Journal of Biomedical and Health Informatics, Jg. 17, Nr. 3, S. 579-590, 2013.

[155] M. Raubal, „Wayfinding in built environments: The case of airports“, IfGIprints, Jg. 14, 2002.

[156] T. Reichenbacher, Mobile cartography: adaptive visualisation of geographic information on mobile devices. Verlag Dr. Hut München, 2004.

[157] Reuters, Amazon.com to buy Kiva Systems for \$7ry million, 2012. Adresse: http : / / ww . reuters . com / article / us - amazoncom idUSBRE82I11720120319.

[158] S. K. Robson, „Areview of psychological and cultural effects on seating behavior and their application to foodservice settings", Journal of Foodservice Business Research, Jg. 5, Nr. 2, S. 89-107, 2002.

[159] P. Ruppel, F. Gschwandtner, C. K. Schindhelm und C. Linnhoff-Popien, "Indoor navigation on distributed stationary display systems", in Proceedings of the 33rd IEEE International Computer Software and Applications Conference (COMPSAC'09), IEEE, 2009, S. 37-44. 
[160] S. Schneider und R. Koenig, „Exploring the Generative Potential of Isovist Fields - The Evolutionary Generation of Urban Layouts based on Isovist Field Properties", in 30th International Conference on Education and Research in Computer Aided Architectural Design in Europe, 2012, S. 355-363.

[161] D. Schultes und P. Sanders, „Dynamic highway-node routing“, in Experimental Algorithms, Springer, 2007, S. 66-79.

[162] C. Seiler und K. Wohlrabe, „Archetypal Scientists“, CESifo Group Munich, CESifo Working Paper Series 3990, 2012.

[163] S. Shekhar, S. K. Feiner und W. G. Aref, „Spatial Computing“, Communications of the ACM, Jg. 59, Nr. 1, S. 72-81, 2016.

[164] J. G. Siek, L.-Q. Lee und A. Lumsdaine, The Boost Graph Library: User Guide and Reference Manual, Portable Documents. Pearson Education, 2001.

[165] R. Snowden, R. J. Snowden, P. Thompson und T. Troscianko, Basic Vision: an Introduction to Visual Perception. Oxford University Press, 2012 .

[166] M. Solé, V. Muntés-Mulero, A. I. Rana und G. Estrada, „Survey on Models and Techniques for Root-Cause Analysis", arXiv preprint arXiv:1701.08546, 2017.

[167] South China Morning Post, Chinese firm halves worker costs by hiring army of robots to sort out 200,000 packages a day, 2017. Adresse: http: //www.scmp.com/news/china/society/article/2086662/chinesefirm-cuts-costs-hiring-army-robots-sort-out-200000.

[168] statista, Das Smartphone (fast) immer im Blick, 2016. Adresse: https: / / de . statista . com / infografik / 4192 / nutzungsmuster - von mobiltelefonnutzern/.

[169] S. Steiniger, M. Neun und A. Edwardes, „Foundations of Location Based Services Lesson 1 CartouCHe 1-Lecture Notes on LBS, V. 1.0“, 2011.

[170] D. Sui, S. Elwood und M. Goodchild, Crowdsourcing Geographic Knowledge: Volunteered Geographic Information (VGI) in Theory and Practice. Springer Science \& Business Media, 2012.

[171] C. Tandy, „The isovist method of landscape survey“, Methods of Landscape Analysis, S. 9-10, 1967.

[172] S. Thrun, ,Learning metric-topological maps for indoor mobile robot navigation", Artificial Intelligence, Jg. 99, Nr. 1, S. 21-71, 1998.

[173] S. Thrun, „Learning Occupancy Grid Maps With Forward Sensor Models", Autonomous Robots, Jg. 15, Nr. 2, S. 111-127, 2003. 
[174] R. Triebel, K. Arras, R. Alami, L. Beyer, S. Breuers, R. Chatila, M. Chetouani, D. Cremers, V. Evers, M. Fiore u. a., „Spencer: A socially aware service robot for passenger guidance and help in busy airports", in Field and Service Robotics, Springer, 2016, S. 607-622.

[175] A. Turner, M. Doxa, D. O'sullivan und A. Penn, „From isovists to visibility graphs: a methodology for the analysis of architectural space", Environment and Planning B: Planning and Design, Jg. 28, Nr. 1, S. 103121, 2001.

[176] M. Vlachos, G. Kollios und D. Gunopulos, „Discovering similar multidimensional trajectories", in Proceedings of the 18th International Conference on Data Engineering, IEEE, 2002, S. 673-684.

[177] D. Wagner, T. Willhalm und C. Zaroliagis, „Geometric containers for efficient shortest-path computation“, Journal of Experimental Algorithmics (JEA), Jg. 10, S. 1-3, 2005.

[178] M. Werner, Indoor Location-Based Services: Prerequisites and Foundations. Springer, 2014.

[179] M. Werner und S. Feld, „Homotopy and Alternative Routes in Indoor Navigation Scenarios", in Proceedings of the 5th International Conference on Indoor Positioning and Indoor Navigation (IPIN 2014), IEEE, 2014, S. 230-238.

[180] M. Werner und M. Kiermeier, „Alow-dimensional feature vector representation for alignment-free spatial trajectory analysis", in Proceedings of the 5th ACM SIGSPATIAL International Workshop on Mobile Geographic Information Systems, ACM, 2016, S. 19-26.

[181] J. M. Wiener und G. Franz, „Isovists as a means to predict spatial experience and behavior", in Spatial Cognition IV: Reasoning, Action and Interaction, Springer, 2004, S. 42-57.

[182] J. M. Wiener, C. Hölscher, S. Büchner und L. Konieczny, „Gaze behaviour during space perception and spatial decision making", Psychological research, Jg. 76, Nr. 6, S. 713-729, 2012.

[183] Wireless Communications and Public Safety Act of 1999, https://www.gpo.gov/fdsys/pkg/BILLS-106s800enr/pdf/BILLS106s800enr.pdf, 1999.

[184] I. H. Witten, E. Frank, M. A. Hall und C. J. Pal, Data Mining: Practical Machine Learning Tools and Techniques. Morgan Kaufmann, 2016.

[185] T. R. Wylie, „The discrete Fréchet distance with applications“, Diss., Montana State University-Bozeman, College of Engineering, 2013.

[186] H.-J. Yu und D.-S. Huang, „Normalized feature vectors: a novel alignment-free sequence comparison method based on the numbers of adjacent amino acids", IEEE/ACM Transactions on Computational Biology and Bioinformatics (TCBB), Jg. 10, Nr. 2, S. 457-467, 2013. 
[187] Y. Zheng und X. Zhou, Computing with spatial trajectories. Springer Science \& Business Media, 2011. 